\title{
Semiconductor Laser Device-Level Characteristics
}

\author{
A Thesis \\ Presented to the Faculty of \\ California Polytechnic State University, \\ San Luis Obispo \\ In Partial Fulfillment \\ of the Requirements for the Degree \\ Master of Science in Electrical Engineering
}

by

Clement Law

May 2011 
(C) 2011

Clement Law

ALL RIGHTS RESERVED 


\section{COMMITTEE MEMBERSHIP}

Title: $\quad$ Semiconductor Laser Device-Level Characteristics

Author:

DATE SUBMITTED:

COMMITTEE CHAIR:

COMMITTEE MEMBER:

COMMITTEE MEMBER:
Clement Law

May 3, 2011
Dr. John Saghri, Associate Professor

Dr. Xiao-Hua (Helen) Yu, Associate Professor 


\section{ABSTRACT \\ Semiconductor Laser Device-Level Characteristics \\ by Clement Law}

High-speed modulations of the semiconductor lasers are highly desirable in cost-effective optical communication systems. Developing the experimental setups to extract the characteristics of the semiconductor lasers is vital to the future of the optical research projects. In this thesis, integrated experimental setup designs have been developed to measure the characteristics of the Vertical Cavity Surface Emitting Laser (VCSEL), Distributed Feedback (DFB), and Fabry-Pérot (FP) lasers. The measurements of the DC characteristics are optical power versus drive current (L-I) curves (DFB, VCSEL) and optical spectra (FP, DFB, VCSEL). In addition, the high-speed optical detection measurement of the optoelectronic frequency responses for VCSEL and FP lasers, and relative intensity noise (RIN) for DFB and FP lasers have also been measured. Finally, the measurement of the frequency response of the optical pumping with 850nm VCSEL has been attempted. 


\section{ACKNOWLEDGEMENTS}

First, I would like to express my sincere appreciation to my thesis advisor, Professor Xiaomin Jin for her assistance in my research and experiments. Though it was a great challenge for me to do research experiments in semiconductor lasers, especially on VCSEL (Vertical Cavity Surface Emitting Laser), Professor Xiaomin Jin's expertise in semiconductor lasers gave me the confidence and support to overcome many difficulties in my research experiments. In addition, I have to thank my relatives and friends throughout the years for their love and support. Furthermore, I would like to thank each of my thesis committee members, Dr. Xiaomin Jin, Dr. John Saghri, and Dr. Xiao-Hua (Helen) Yu for commenting on my thesis. Finally, I would like to dedicate this thesis to my parents; their never-ending love and support throughout the years have also contributed to this thesis. 


\section{TABLE OF CONTENTS}

Page

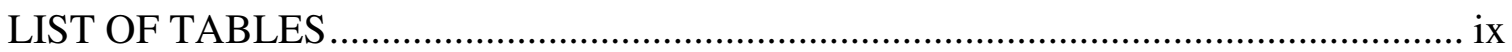

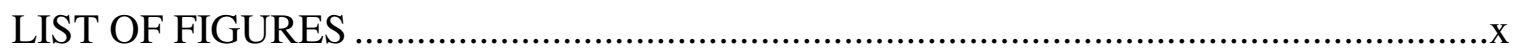

Chapter 1: Introduction ....................................................................................

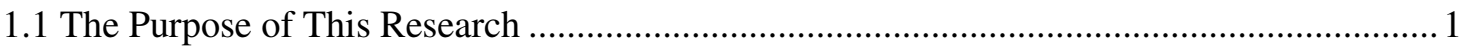

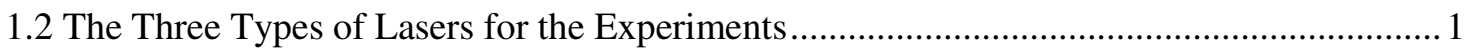

1.2.1 Introduction of Fabry-Pérot (FP) Laser ............................................................... 2

1.2.2 Introduction of Distributed Feedback (DFB) Laser ..................................................... 3

1.2.3 Introduction of Vertical-Cavity Surface-Emitting Laser (VCSEL) ..............................

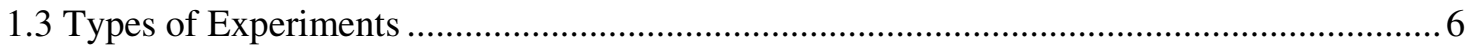

1.3.1 DC L-V-I (Light Voltage Current) Laser Measurement .............................................. 6

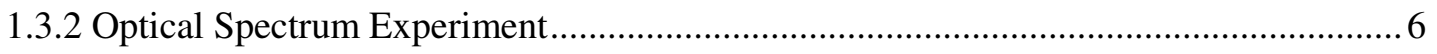

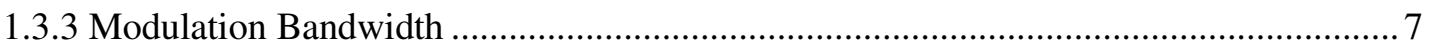

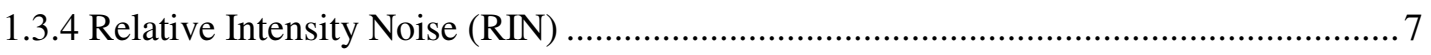

1.3.5 Frequency Response of VCSEL Optical Pumping .................................................. 8

Chapter 2: Laser DC Measurement .................................................................

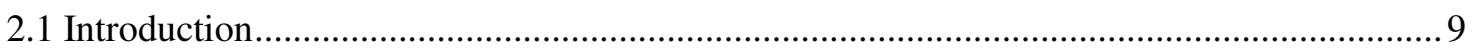

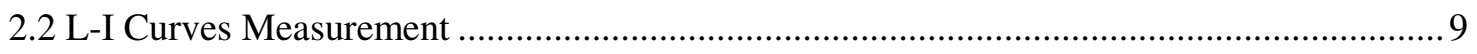

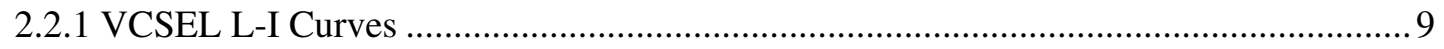




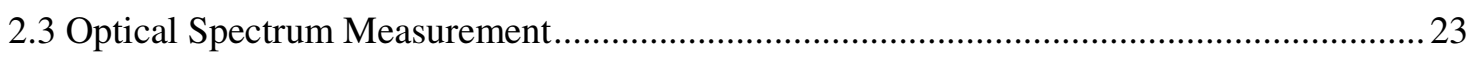

2.3.1 Background in Optical Spectrum Analysis ........................................................... 23

2.3.2 Fabry-Pérot Optical Spectrum Experimental Setup and Results.................................24

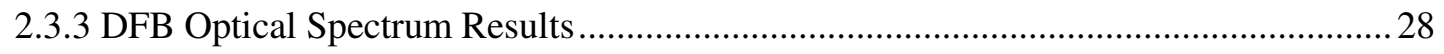

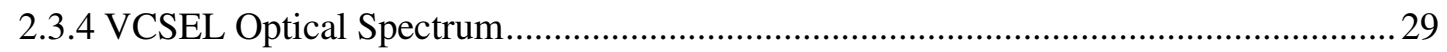

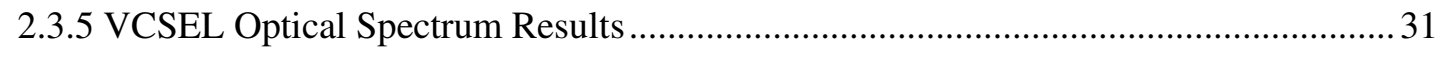

Chapter 3: Frequency Response for High Speed Measurement of Lasers .......................33

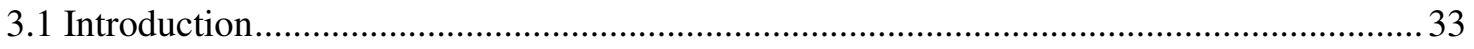

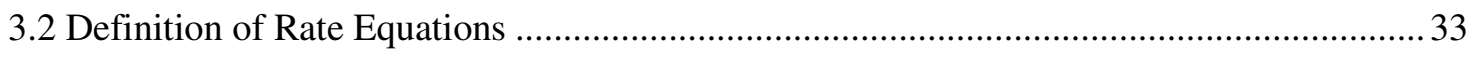

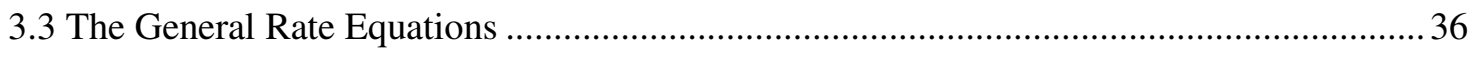

3.3.1 Stimulated Emission - Absorption and Gain........................................................ 37

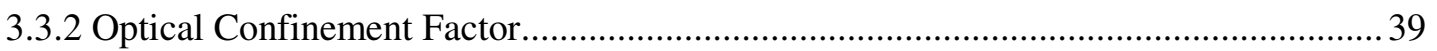

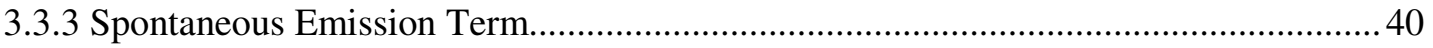

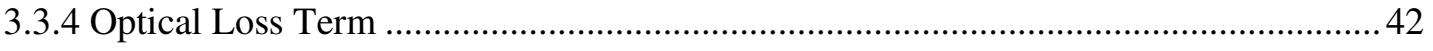

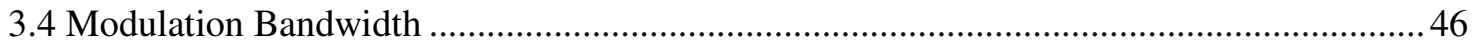

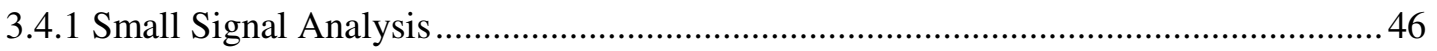

3.5 High Frequency Bandwidth Experimental Results .......................................................53

3.5.1 VCSELs High Frequency Bandwidths Experimental Results ...................................53

3.5.2 Fabry-Pérot High Frequency Bandwidths Experimental Results..................................58 
Chapter 4: Relative Intensity Noise (RIN)

4.1 Introduction of Relative Intensity Noise. 62

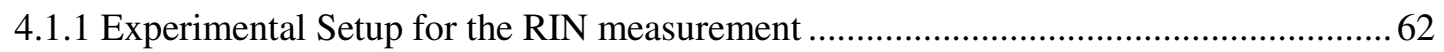

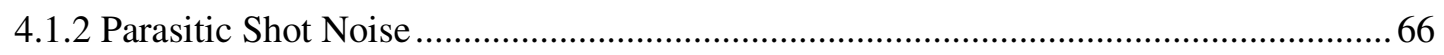

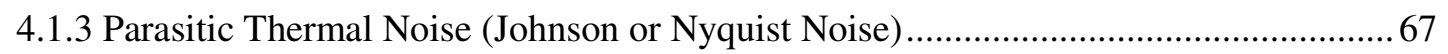

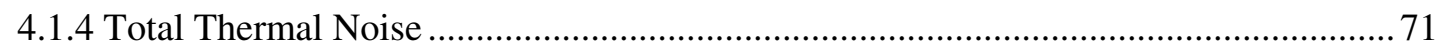

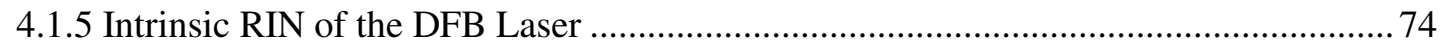

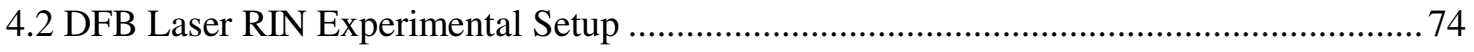

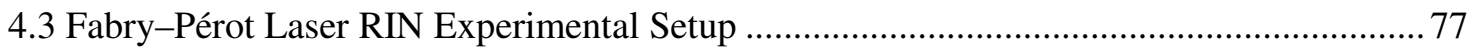

Chapter 5: Frequency Response of VCSEL under Optical Pumping .............................83

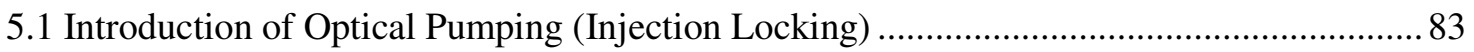

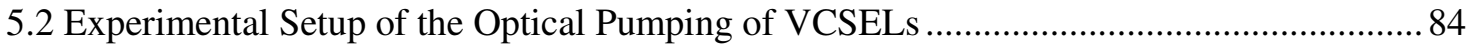

Chapter 6: Conclusions and Future Works ............................................................97

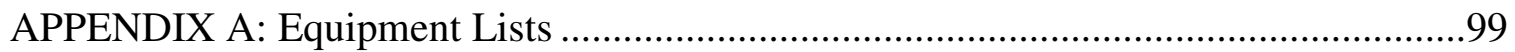

APPENDIX B: Algorithm for Indexing and Reading from the Indexed Optical Power

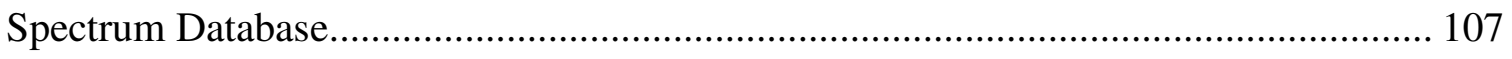

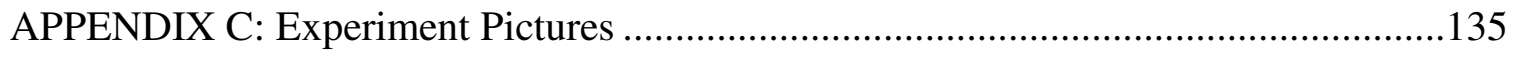

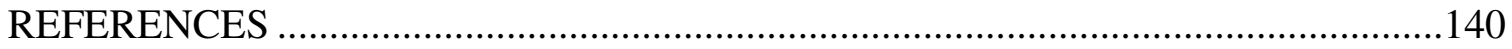




\section{LIST OF TABLES}

Table Page

2-1 Four HP Fabry-Pérot lasers OSA parameters settings............. 25

4-1 Important specifications of the RIN measurement setup........... 63

4-2 Calibrated Power at 6.35 GHz for the DFB laser RIN plots......... 75

4-3 Amplifiers Parameters for Fabry-Pérot laser RIN measurement..... 77 


\section{LIST OF FIGURES}

Figure Page

1-1 Fabry-Pérot resonator multiple internal reflections from two partially reflective

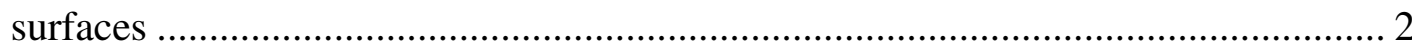

1-2 Distributed feedback (DFB) semiconductor laser schematic diagram .................. 4

1-3 Bragg diffraction grating with incident rays being scattered from successive

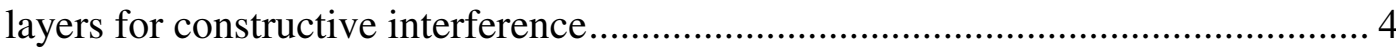

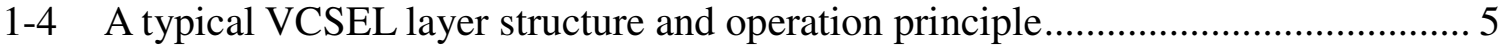

2-1 Block diagram to measure the VCSEL DC L-V-I curves .................................. 10

2-2 The rear of LDM-4407 ILX Lightwave Laser Diode Mount configurations ..........11

2-3 HFE4192-901 850nm VCSEL pins ................................................................ 13

2-4 The 850nm VCSEL connected to LDM-4407 ............................................... 13

2-5 Front panel of LDC-3744B laser diode controller .......................................... 13

2-6 Back panel of LDC-3744B laser diode controller ........................................... 14

2-7 9-Pin connector of LDC-3744B laser diode controller ..................................... 14

2-8 9-pin connector of LDM-4407 temperature controlled mount ............................ 14

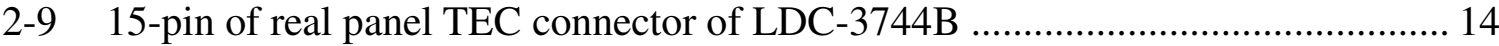

2-10 9-pin of male D TE of LDM-4407 temperature controlled mount ........................ 14

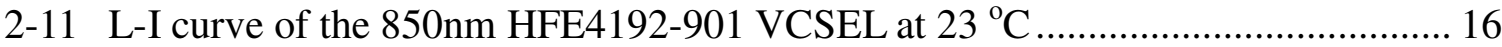

2-12 L-I curves of the HFE4192-901 VCSEL at various temperatures ........................ 17

2-13 Threshold currents of the HFE4192-901 VCSEL at various temperatures............. 18 
2-14 VCSEL 850nm Voltages vs. Input Currents at Various Temperatures .................... 18

2-15 Kette Laser Diode Driver and Temperature Controller, KNN-2000LT ................. 19

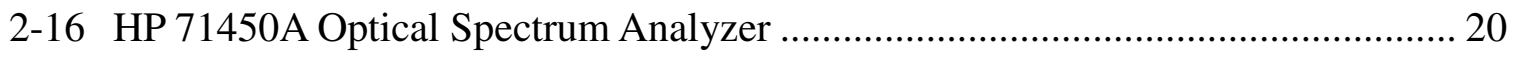

2-17 Block Diagram of the DFB Laser Optical Power Measurement via OSA ............ 20

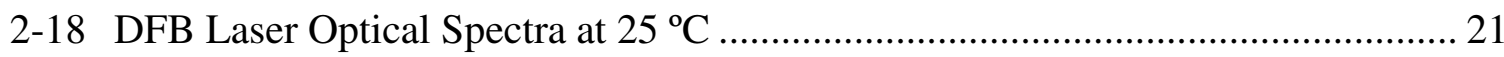

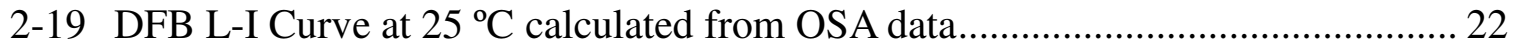

2-20 DFB Laser L-I Curve at Various Temperatures calculated from OSA data ............ 22

2-21 Block diagram of Fabry-Pérot optical spectrum experiment setup..................... 25

2-22 (a), (b), (c), (d) The optical spectra of HP Fabry-Pérot lasers ........................ 26,27

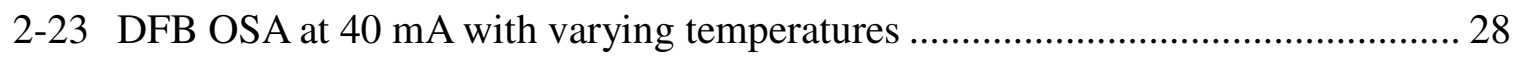

2-24 Block Diagram of Optical Spectrum Analysis of the 850nm VCSEL ................... 29

2-25 VCSEL OSA at $16 \mathrm{~mA}$ with varying temperatures......................................... 31

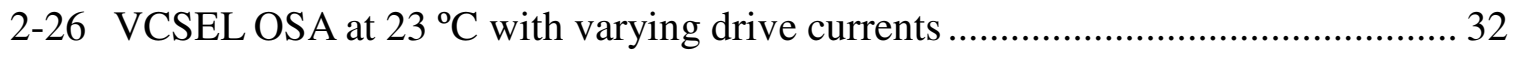

3-1 Two mechanisms included in the optical loss term in Equation (3.2.4) ................ 36

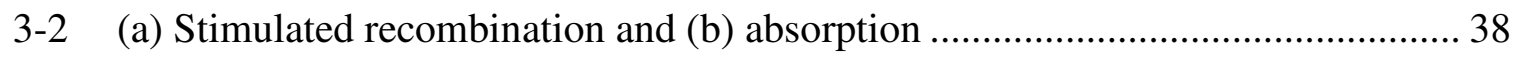

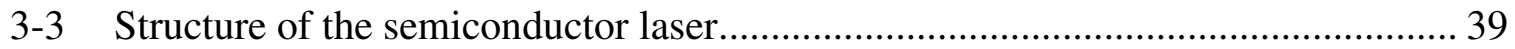

3-4 Carrier recombination mechanism in semiconductor laser [9] .......................... 41

3-5 The effect of the gain medium on the optical power of a beam making a round-trip in the Fabry-Pérot laser cavity. ................................................ 45

3-6 Flow Chart of Derivation of the Transfer Function from Rate Equations .............. 48

3-7 Frequency response of DFB laser at different output powers............................ 53

3-8 Block Diagram of the VCSEL bandwidth measurement ................................... 54 
3-9 Frequency response of the default-power VCSEL ....................................... 54

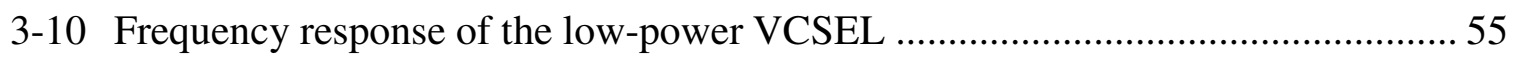

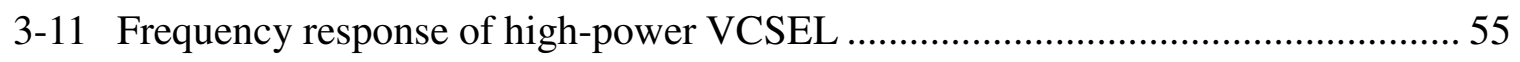

3-12 Optical bandwidths of the default-power VCSEL at 850nm ............................ 56

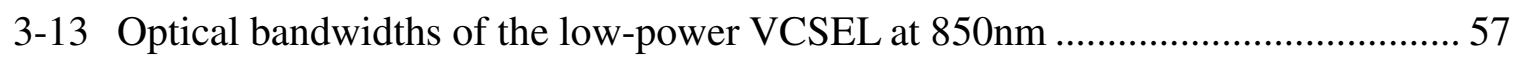

3-14 Optical bandwidths of the high-power VCSEL at 850 nm .............................. 57

3-15 Block diagram of the Fabry-Pérot bandwidth measurement .............................. 58

3-16 Frequency response of \#1 Fabry-Pérot laser, The bandwidth is about $3.16 \mathrm{GHz} . .59$

3-17 Frequency response of \#2 Fabry-Pérot laser. The bandwidth is $3.31 \mathrm{GHz} . . . . . . . . .59$

3-18 Frequency response of \#3 Fabry-Pérot laser. The bandwidth is 5.05 GHz........... 60

3-19 Frequency response of \#4 Fabry-Pérot laser. The bandwidth is $7.88 \mathrm{GHz} . . . . . . . . . .60$

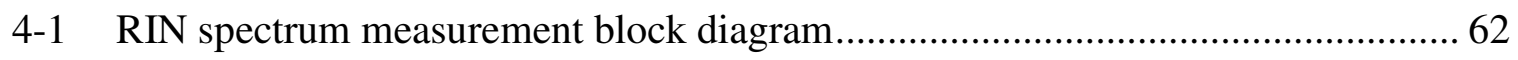

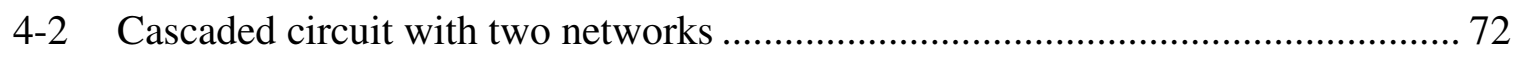

4-3 Block diagram of DFB laser relative intensity noise (RIN) ............................ 75

4-4 RIN plots of the DFB laser at various input currents from 5mA to 48mA ............ 76

4-5 Block diagram of the Fabry-Pérot laser experimental setup.............................. 77

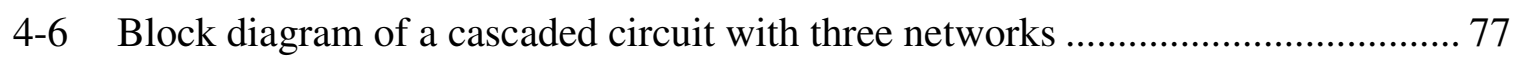

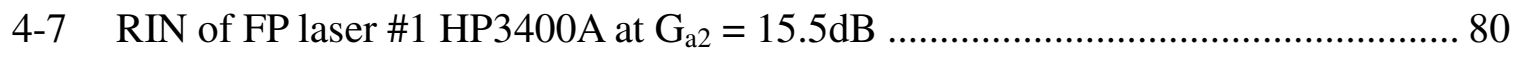

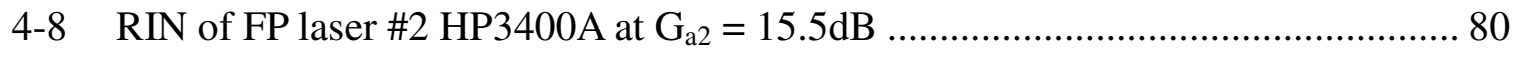

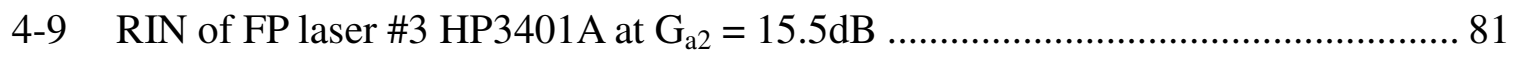

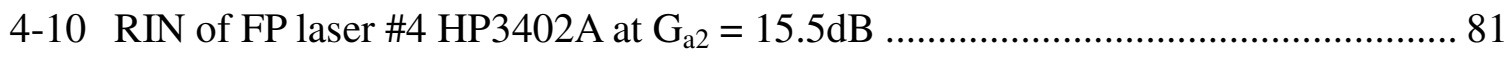

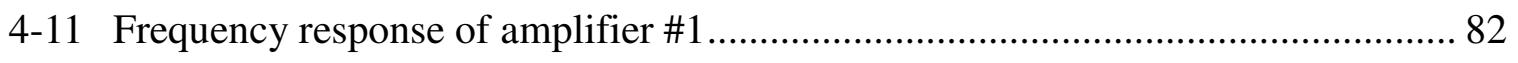




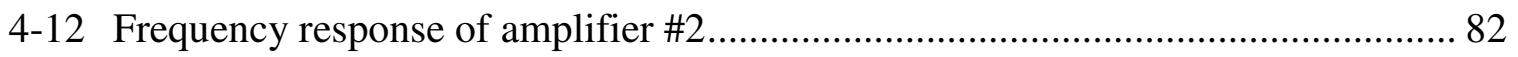

5-1 Experimental setup of injection locking for the 850nm VCSEL. Both the master and slave lasers are VCSELs ................................................................... 86

5-2 Biased Tee connection with RF signal from port 1 of HP 8720B Network Analyzer, DC biased signal from the Current Source ILX Lightwave LDX-3207 and the VCSEL with a modified pin is soldered onto the SMA connector outputting the DC biased, RF + DC signal into the coupler ............................. 86

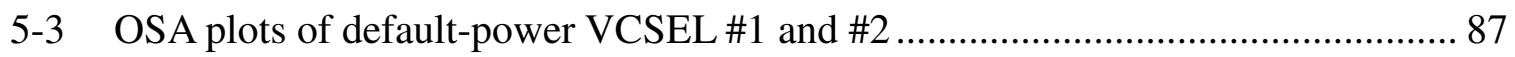

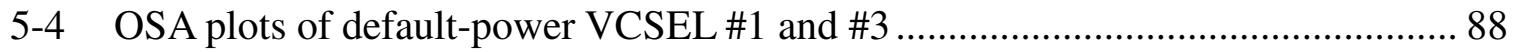

5-5 Test \#1: The optical spectra of the VCSELs from 2mA to 20mA.

The average peak of the optical spectra is $-51.72 \mathrm{dBm}$ 89

5-6 Test \#2: The optical spectra of the VCSELs from 2mA to 20mA.

The average peak of the optical spectra is $-29.552 \mathrm{dBm}$ 90

5-7 Test \#3: The optical spectra of the VCSELs from 2mA to 20mA.

The average peak of the optical spectra is $-50.806 \mathrm{dBm}$ 90

5-8 Test \#4: The optical spectra of the VCSELs from 2mA to 20mA.

The average peak of the optical spectra is $-30.084 \mathrm{dBm}$ 91

5-9 Test \#1: The optical spectra of the VCSELs after optical pumping from $2 \mathrm{~mA}$ to $20 \mathrm{~mA}$. The average peak of the optical spectrum is $-26.664 \mathrm{dBm}$

5-10 Test \#2: The optical spectra of the VCSELs after optical pumping from $2 \mathrm{~mA}$ to $20 \mathrm{~mA}$. The average peak of the optical spectrum is $-28.164 \mathrm{dBm}$ 92

5-11 Test \#1: The frequency responses are noisy when the test laser is connected to the 
$50 \%$ port with master laser turned off.

5-12 Test \#2: The frequency responses are noisy when the test laser is connected to the $50 \%$ port with the master laser at the $100 \%$ port of the coupler

5-13 Test \#3: The frequency responses are visible when the test laser is connected to the $100 \%$ port coupling with the output at the $50 \%$ while the master laser is turned off.

5-14 Test \#4: The frequency responses are visible when the test laser is connected to the $100 \%$ port with the master laser turned on at the $50 \%$ port. 


\section{Chapter 1: Introduction}

\subsection{The Purpose of This Research}

The goal of this research is to study three types of semiconductor laser characteristics. This is done by designing the experimental setup which also contributes to the foundation for the upcoming optical research in the university's laboratory. Three types of lasers are studied, namely, Fabry-Pérot (FP) lasers at 1300nm, distributed feedback (DFB) lasers at $1550 \mathrm{~nm}$, and vertical-cavity surface-emitting lasers (VCSELs) at 850nm. The experiments utilize the computer software, LabVIEW, which runs in Windows operating system in PC to control multiple lab equipment simultaneously to measure the properties of different types of lasers to perform data analysis.

\subsection{The Three Types of Lasers for the Experiments}

There are three types of lasers that are being used for the experiments. The FP and DFB are edge emitting lasers while the VCSELs are surface emitting lasers. The coupling efficiency depends on the radiation pattern of an emitter. Surface emitting lasers have circularly symmetric emission profile which results in low-cost solutions by avoiding external optics [1]. The edge emitting lasers concentrate their radiation more than surface emitting devices which provide improved coupling efficiency. 


\subsubsection{Introduction of Fabry-Pérot (FP) Laser}

Fabry-Pérot laser consists of a resonator region bounded by two parallel partially reflecting mirrors with separation distance $L$. The beam arrives at the first surface, $\mathrm{M}_{1}$. A portion of the light incident upon the surface will be reflected and a portion will be transmitted. The transmitted beam of light continues to reach the second surface, $\mathbf{M}_{2}$, which is also partially reflected and partially transmitted. The reflected beam continues to bounce back and forth between $\mathrm{M}_{1}$ and $\mathrm{M}_{2}$ to form a standing wave. The total transmitted beams are shown in Figure 2-26. To produce a standing wave pattern, the cavity must be an integral number of half wavelengths long as shown below.

$$
L=\frac{m \lambda}{2}
$$

where $\lambda$ is the wavelength as measured in the material within the cavity and $m$ is the positive integer.

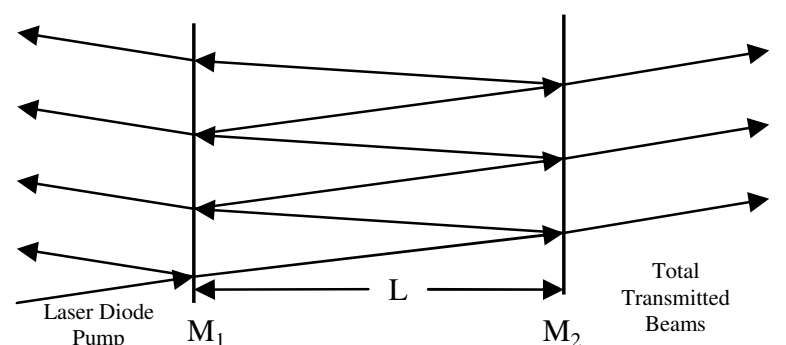

Figure 1-1: Fabry-Pérot resonator multiple internal reflections from two partially reflective surfaces

The cavities are resonant at a number of wavelengths or frequencies at shown in Equation (1.2.1). The resonant frequencies can be found by:

$$
f=\frac{m v}{2 L}=\frac{m c}{2 L n}
$$

where $c$ is the speed of light $3 \times 10^{8} \mathrm{~m} / \mathrm{s}, v=c / n$ is the beam velocity depending on the 
index of refraction, $n$ of the material. In frequency space, the adjacent cavity longitudinal modes is

$$
\Delta f_{c}=\frac{c}{2 L n}
$$

The corresponding free-space wavelength spread $\Delta \lambda_{c}$, can be found by the relationship $\frac{\Delta f_{c}}{f}=\frac{\Delta \lambda_{c}}{\lambda_{0}}$, where $\lambda_{0}$ is the free-space value of the mean wavelength and $f=c / \lambda_{0}$ is the mean frequency.

$$
\Delta \lambda_{c}=\frac{\lambda_{0}^{2}}{c} \Delta f_{c}
$$

The Fabry-Pérot laser output power optical spectrum thus should contain multiple modes as shown in Chapter 2.

\subsubsection{Introduction of Distributed Feedback (DFB) Laser}

Unlike Fabry-Pérot laser which uses two mirrors to form the optical cavity, distributed feedback laser has a periodic index distribution layer or diffraction grating etched internally just above the active region. This grating is a distributed filter which allows only one of the cavity's longitudinal modes to propagate back and forth (see Figure 1-2). Distributed feedback laser diode thus has a narrow-spectral-width with a singlelongitudinal-mode. 


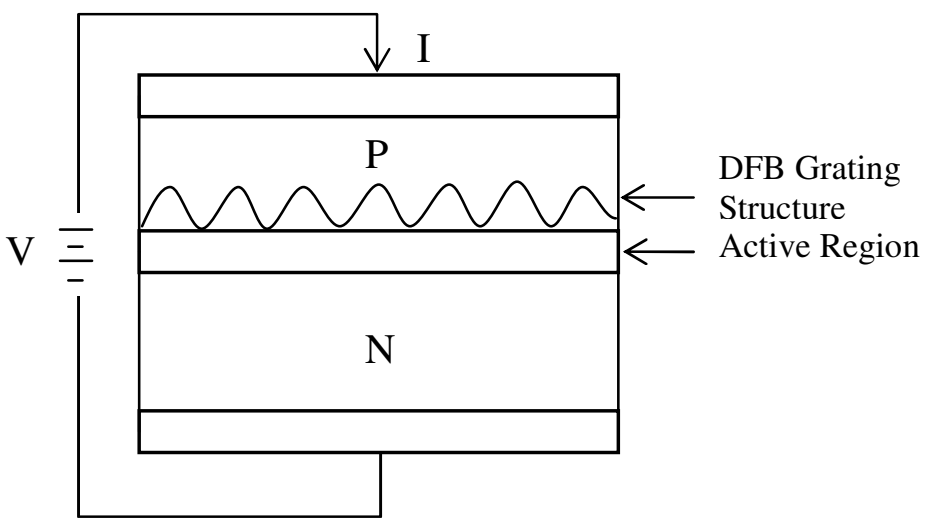

Figure 1-2: Distributed feedback (DFB) semiconductor laser schematic diagram

The diffraction gratings are based on Bragg's Law. Bragg gratings have multiples of half wavelengths, so that the incident light rays are scattered and reflected by each grating due to the internal reflections (see Figure 1-3). The spacing between the gratings is defined by

$$
\Lambda=\frac{m \lambda}{2 \sin (\pi / 2)}=\frac{m \lambda_{0}}{2 n}
$$

The wavelength in the material is related to the free-space wavelength by

$$
\lambda=\frac{\lambda_{0}}{n}
$$

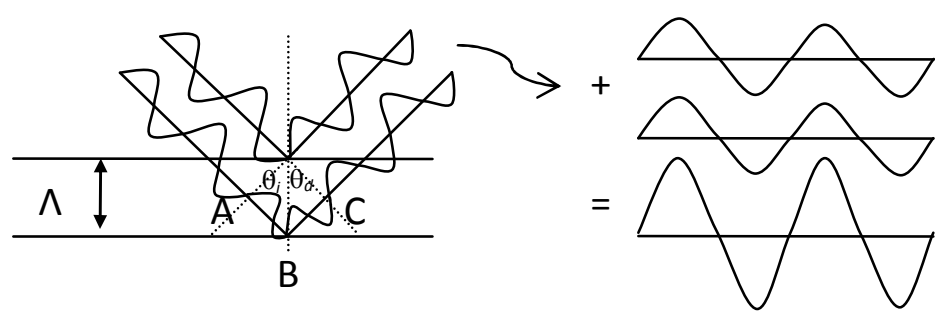

Figure 1-3: Bragg diffraction grating with incident rays being scattered from successive layers for constructive interference

The light rays that are being scattered by the gratings form constructive interference at the output of the DFB laser with a single longitudinal mode. 


\subsubsection{Introduction of Vertical-Cavity Surface-Emitting Laser (VCSEL)}

The vertical-cavity surface-emitting laser (VCSEL) is a type of semiconductor laser diode with laser beam emission perpendicular from the top of the surface. This is contrary to FP and DFB edge-emitting lasers. One of the advantages of VCSEL over conventional edge-emitting lasers is to test the unit without waiting until the end of the production process. Figure 1-4 illustrates the typical layout of a VCSEL.

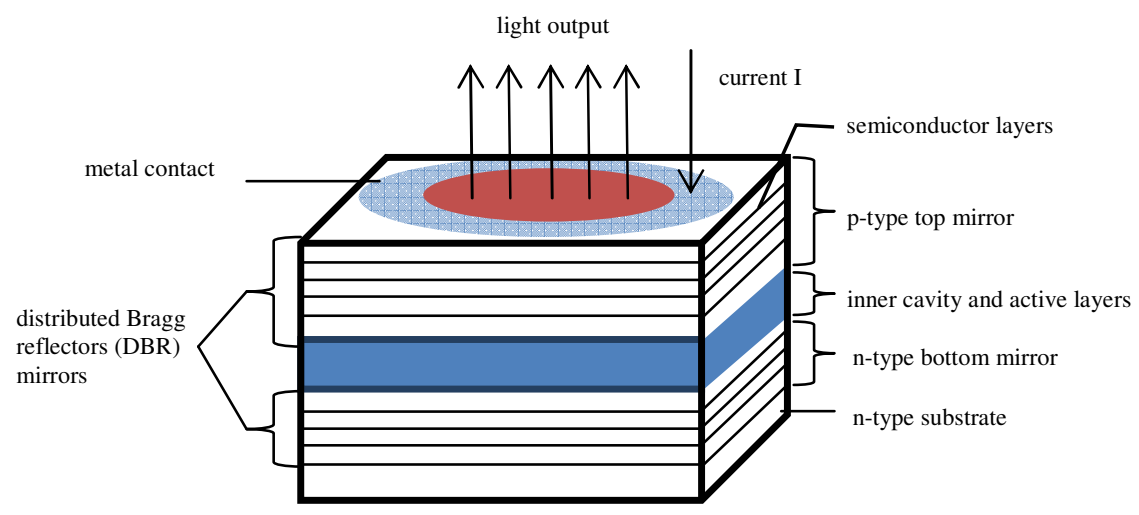

Figure 1-4: A typical VCSEL layer structure and operation principle

The resonator of a VCSEL consists of two distributed Bragg reflector (DBR) mirrors that are sandwiching and parallel to the active region which consists of one or more quantum wells for generating laser light. The top and bottom mirrors are doped as p-type and ntype to form a diode junction. Each DBR mirror is made from a stack of semiconductor layers with alternating high and low refractive indices with quarter-wavelength thickness. When all of the waves that are reflected from the mirrors interfere constructively, there will be high total reflectivity with a phase change on reflection of exactly zero or $\pi$ [22]. The light waves that are emitted in the active region are reflected by the top and bottom mirrors for lasing. 


\subsection{Types of Experiments}

\subsubsection{L-V-I (Light Voltage Current) Laser Measurement}

It is a study of the laser diode output optical power by varying the input current. The power versus current relationship shows that when the laser operates linearly and coherently, the current versus voltage measurement tells us which threshold voltage brings the current to its operating point. These results are obtained using a laser diode controller, photo detector, optical power meter, and a computer to record the data. By varying the temperature of the laser diode using a temperature controller, the changes in the L-V-I relationships are also studied.

\subsubsection{Optical Spectrum Experiment}

The solid state lasers used in the experiments utilize the band gaps at the $p-n$ junctions of the semiconductor material to emit photons at the active region. In general, the electric current is injected to supply the electrons for the active region for recombination where electrons fall from high energy states to low energy states to emit photons. These emitted photons are wave-like particles which have different frequencies or wavelengths, serve as feedback within the active region to stimulate more injected electrons into the excited states for stimulated emissions. These stimulated emitted photons are coherent to each other and should ideally have a single wavelength, which is monochromatic. In reality, even a very coherent laser source still produces radiation over a range of wavelengths. These wavelengths can be measured and recorded using the optical spectrum analyzer. 
Temperature change induces shifting of the center wavelength of a laser. This is due to the temperature dependence of refractive indices of the laser materials. The resonant wavelengths and the spacing of adjacent resonant wavelengths are determined by the refractive index of the cavity. The change in temperature varies the guiding layer refractive index, which results in a shift of the center wavelength and a change to the spacing of the longitudinal modes of a multimode laser diode. [2]

\subsubsection{Modulation Bandwidth}

This experiment is to study the optical response of a laser under small sinusoidal changes of bias current. The rate equations are used to derive the response function at multiple optical power outputs. The bandwidths of the VCSEL and Fabry-Pérot lasers have been tested and examined. The experimental setup has the frequency responses recorded by LabVIEW for data analysis.

\subsubsection{Relative Intensity Noise (RIN)}

The relative intensity noise describes the noise emitted by the laser. In this experiment, the RIN of the DFB and Fabry-Pérot (FP) lasers are being tested.

$R I N=\frac{\text { Average noise power of laser }}{(\text { Average optical power output })(\text { bandwidth })}$ and its unit is $\mathrm{dB} / \mathrm{Hz}$.

The spectrum analyzer is used to measure not only the laser power output but also the parasitic noises such as the shot noise and thermal noise in the photodetector, broadband amplifier, and inside of the spectrum analyzer. Therefore, a special experimental setup 
and post data processing are required to extract the intrinsic RIN of the laser and eliminating the noises.

\subsubsection{Frequency Response of VCSEL Optical Pumping}

Optical pumping is usually used to improve the bandwidth of the slave or test laser. It uses a master laser to optically pump photons into the slave or test laser. The competition between the amplified spontaneous emissions and the amplified pump laser changes the gain and refractive index according to the carrier density. This allows the master laser to lock the slave laser's optical frequency and to achieve higher gain and sharper optical spectrum. We try to investigate whether optical pumping will improve the laser bandwidth. 


\section{Chapter 2: Laser DC Measurement}

\subsection{Introduction}

This experiment measures the optical power output from the tested lasers as functions of their drive currents in order to study the threshold currents and the operational ranges at various temperatures. There are two types of lasers: Vertical Cavity Surface Emitting Laser (VCSEL) and edge emitting lasers. LabVIEW provides a graphical programming environment for developing sophisticated measurement, test, and control systems. LabVIEW is used to integrate with multiple hardware devices simultaneously to control the laboratory instruments, and record large amounts of data, such as optical power output as a function of drive current at various temperatures, which usually take hours or days of repetitive manual labor.

\subsection{L-I Curves Measurement}

\subsubsection{VCSEL L-I Curves}

In this experiment, a LabVIEW code is developed to control two optical instruments, ILX Lightwave LDC-3744B Laser Diode Controller and Newport Power Meter 835, automatically via GPIB (General Purpose Interface Bus). The ILX Lightwave LDC3744B is a combination of current source and temperature controller for the laser diode. The current source provides a high stability output with a current limit and multiple laser protection features. The built-in temperature controller works with the ILX LDM-4407 Temperature Controlled Laser Diode Mount to deliver precise laser temperature control. 
The Newport Power Meter 835 measures the optical power output from the laser diode.

The optical power values are then recorded by the computer.

Figure 2-1 shows the experimental set-up block diagram to measure the output power as a function of drive current, which is the light versus current (L-I) curve. Appendix A presents the equipment list for the DC measurement experiment.

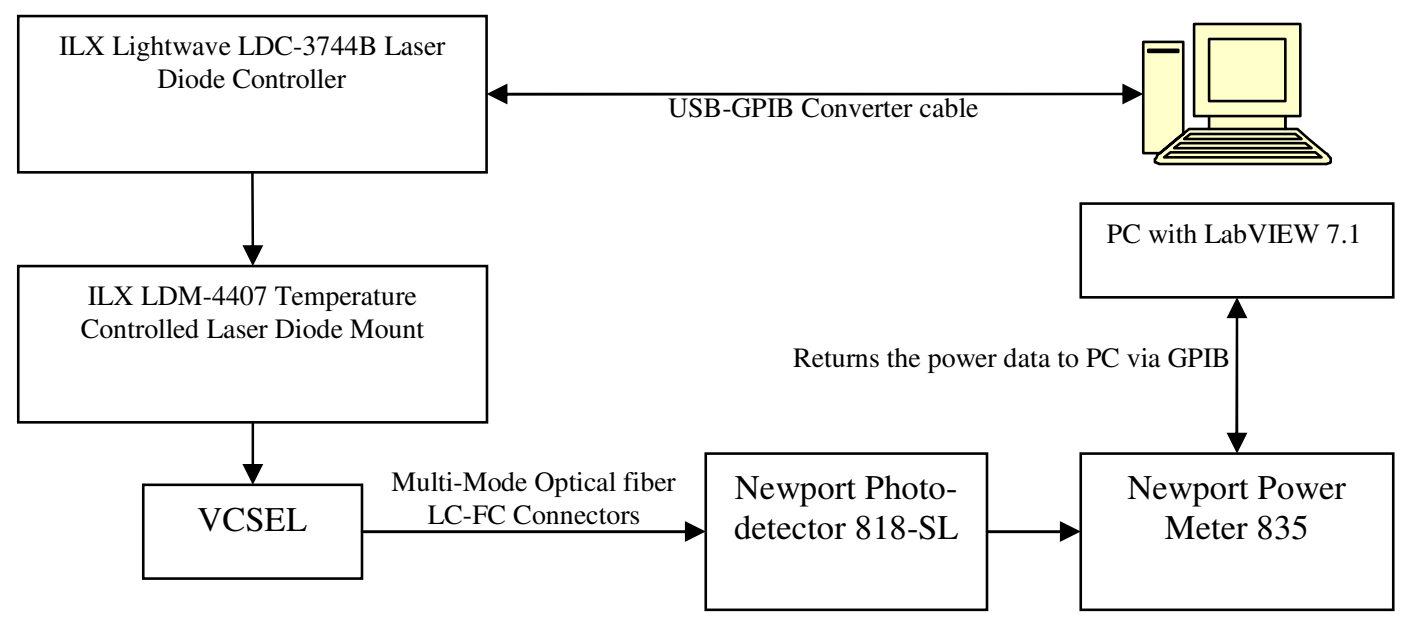

Figure 2-1: Block diagram to measure the VCSEL DC L-V-I curves

The LabVIEW code controls both the ILX Lightwave LDC-3744B Laser Diode Controller and ILX LDM-4407 Temperature Controlled Laser Diode Mount to turn on and change the temperatures of the laser diode via GPIB respectively. The switch settings on the LDM-4407 in Figure 2-2 show the pin configuration for the VCSEL. 


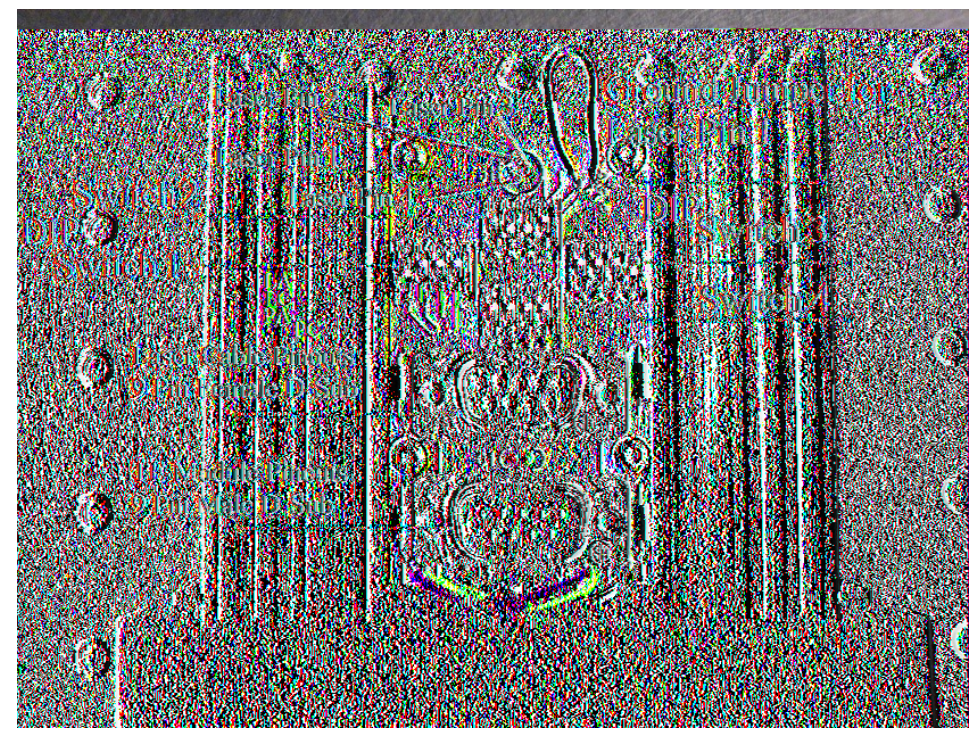

Figure 2-2: The rear of LDM-4407 ILX Lightwave Laser Diode Mount configurations

One of the tested lasers, Honeywell HFE4192-901, a high-performance 850nm multimode oxide-isolated VCSEL, with LC (Local Connector) TOSA (Transmitter Optical Subassembly), is designed for high-speed data communications at 4.25 Gbps. The VCSEL produces circularly symmetric, narrow divergence beams that couple the optical fiber with all of the emitter power. This VCSEL laser is designed to interface with 50/125 and 62.5/125 $\mu \mathrm{m}$ multi-mode fiber [3].

Figure 2-3 shows the three pins of the 850nm HFE4192-901 VCSEL. The VCSEL pin 1 is the anode, pin 2 is the cathode/monitor diode anode, and pin 3 is the monitor diode cathode. The three pins of the VCSEL are connected to the three laser pins at the back of the PCB board of the LDM-4407 that are shown in Figure 2-2. The LDM-4407 must be configured for the particular laser before operation as shown in Figure 2-2. Amongst manufacturers there is no standard procedure for numbering of the laser pins. Thus, ILX 
Lightwave has adopted a generic scheme which coincides with most packages. Figure 22 shows the switch settings for the three-pin VCSEL. According to the data sheet of the Honeywell HFE4192-901 VCSEL, pin 1 is laser anode, LA; pin 2 is laser cathode, LC, and photodiode anode, PA; pin3 is photodiode cathode, PC. Therefore, from Figure 2-2, Switch 1 has the LA position 1 on, Switch 2 has both the LC and PA positions 2 and 3 on, and Switch 3 has the PC position 4 on. Switch 4 is not used and all positions are off. As shown in Figure 2-2, laser pin 1 is identified as the left pin when the package is oriented with the indexing notch up. Pin 2 is the top most pin, pin 3 and pin 4 are numbered in clockwise order. The DIP switches are numbered in the same order as the laser pins. The index on each position of the DIP switch corresponds to a laser function. Position 1 is laser anode, LA; Position 2 is laser cathode, LC; Position 3 is photodiode anode, PA; Position 4 is photodiode cathode, PC. The switch is on when it is positioned up, otherwise it is off. Each switch can independently connect its laser pin to laser anode, laser cathode, photodiode anode, and photodiode cathode. The laser pins of LDM-4407 are electrically isolated from the mount when shipped from the factory. As shown in Figure 2-2, the orientation of the grounding pad area is identical to the laser socket orientation. A wire is connected between the inner laser pad and the outer ring of ground pads to ground pin 1 of the laser.

To power up the laser diode, a 9-pin male-to-male cable is connected between the 9-pin connector (marked Current Source), which provides the drive current for the laser diode, at the back of LDC-3744B Laser Diode Controller in Figure 2-6, and the back of the 
LDM-4407 Laser Cable Pinouts in Figure 2-2. The pins of the current source of LDC3744B and LDM-4407 are shown in Figure 2-7 and Figure 2-8 respectively. The rear panel of the LDC-3744B has a 15-pin female connector marked TE CONTROLLER in Figure 2-9. This TE CONTROLLER connects to the thermoelectric (TE) module of the LDM-4407 laser mount, which has a 9-pin male connector as shown in Figure 2-10 to control the temperature of the laser diode. A 15-pin to 9-pin male-to-female connector cable has to be constructed by matching pins (1,2 TE module +$),(3,4$ TE module -), and (7,8 Temperature Sensor + and -) in order to connect the LDC-3744B and LDM-4407 together for temperature control.
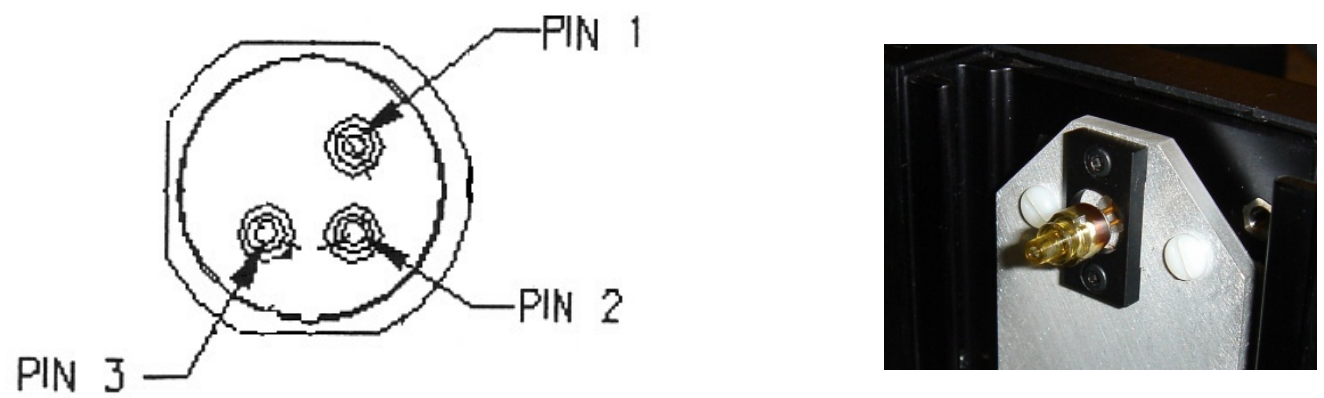

Figure 2-3: HFE4192-901 850nm VCSEL pins

Figure 2-4: The 850nm VCSEL connected to LDM4407

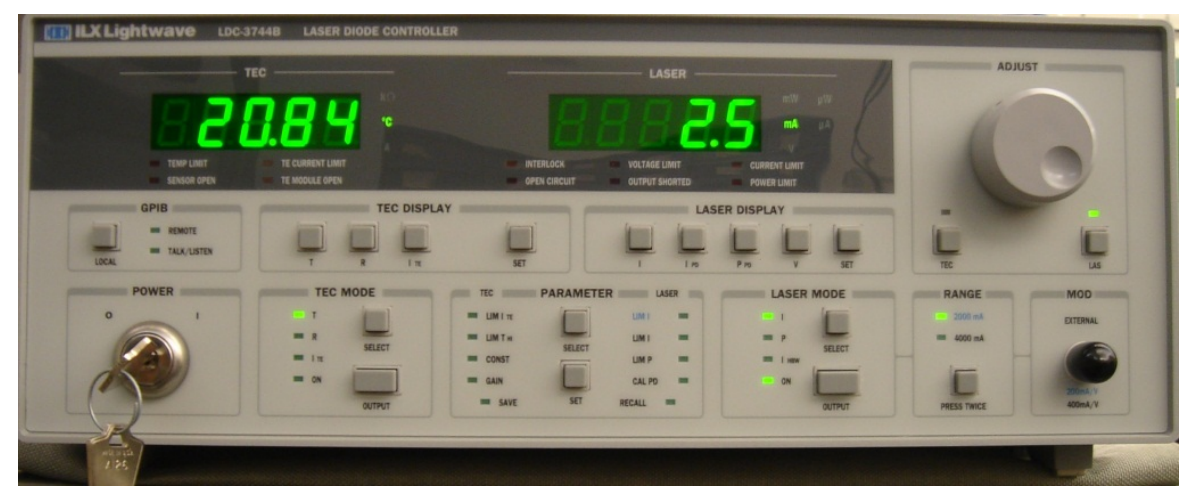

Figure 2-5: Front panel of LDC-3744B laser diode controller 


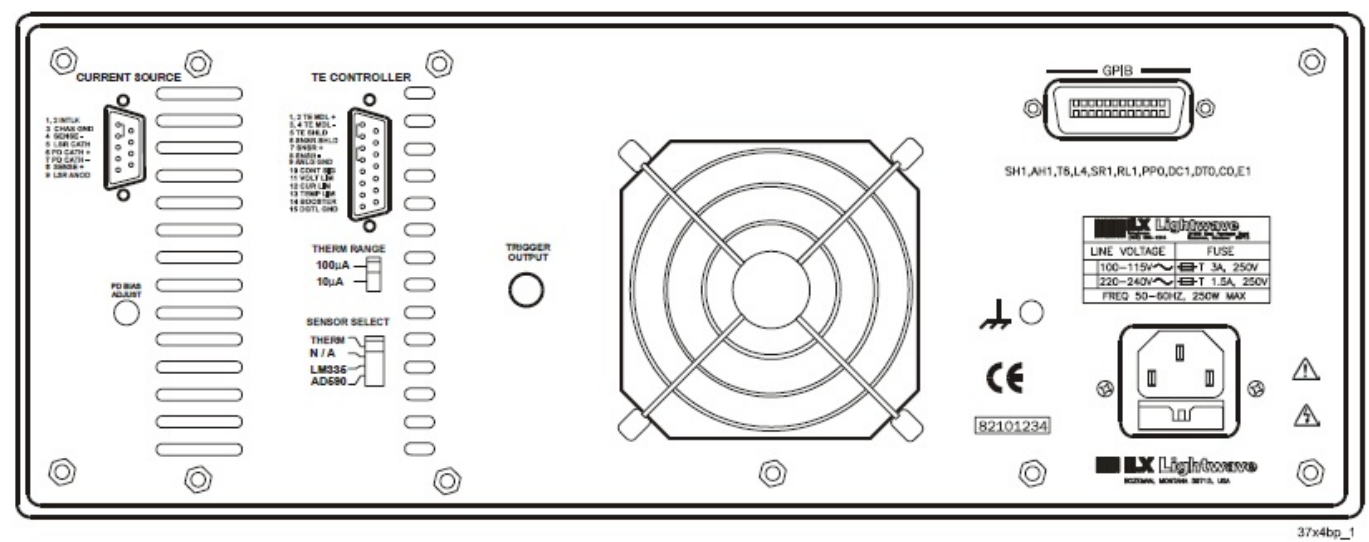

Figure 2-6: Back panel of LDC-3744B laser diode controller

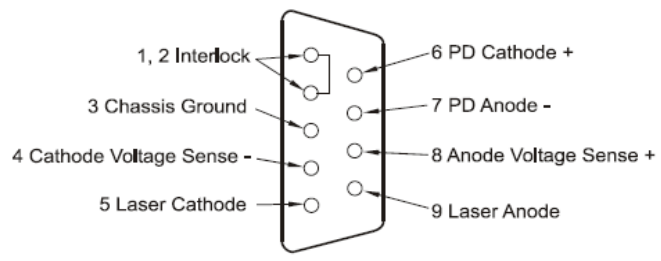

csomn-2

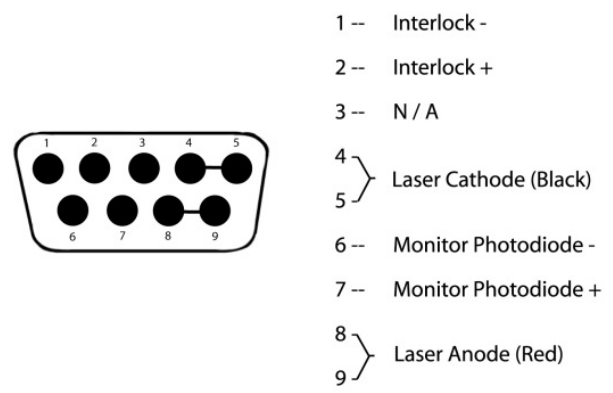

Connector Type : 9 pin female D
Figure 2-7: 9-Pin connector of LDC-3744B laser diode controller

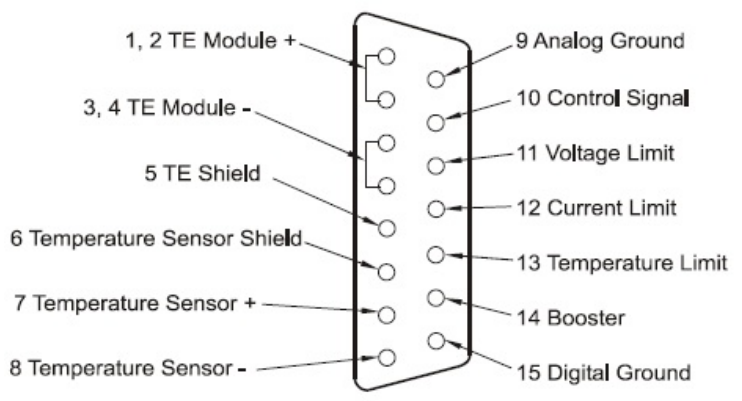

Figure 2.3 Rear Panel TEC Connector

Figure 2-9: 15-pin of real panel TEC connector of $L D C-3744 B$
Figure 2-8: 9-pin connector of LDM-4407 temperature controlled mount

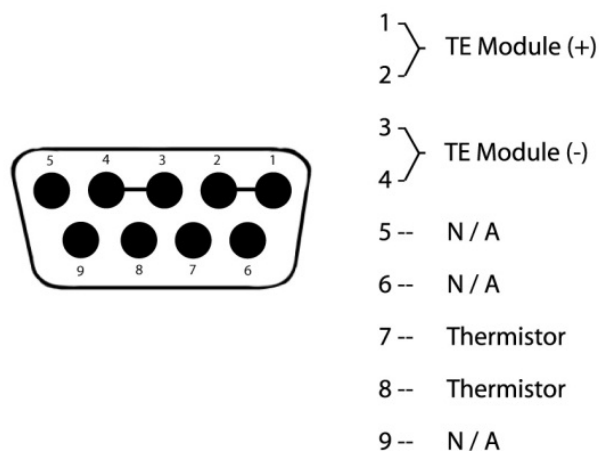

Connector Type : 9 pin male D

Figure 2-10: 9-pin of male D TE of LDM-4407 temperature controlled mount

The LC TOSA plastic barrel of the VCSEL as shown in Figure 2-4 is connected to the Newport Photodetector 818-SL's FC (Fixed Connection) connector that interfaces with 
62.5/125 $\mu \mathrm{m}$ multi-mode optical fiber with LC-FC connectors. The Photodetector 818SL is then connected to the Newport Power Meter 835 which is then connected to the computer running LabVIEW via National Instrument GPIB-USB-HS, which is a GPIB to USB interface.

The optical power outputs that are captured by the Newport Power Meter 835 and the drive current data are at different temperatures range from $-2{ }^{\circ} \mathrm{C}$ to $73{ }^{\circ} \mathrm{C}$ with increments of $5{ }^{\circ} \mathrm{C}$. For each temperature increment, there is a two second delay for the temperature to be stable that falls within $+/-0.5^{\circ} \mathrm{C}$ from the designated temperature. Since the optical power output fluctuates when being detected by the Newport Power Meter 835, there is an algorithm within the automation code to make sure the optical power to be stable before the data is being captured. The algorithm takes 10 power values from Newport 835 power meter at a time and takes the derivative of the power values as shown in Equation (2.1).

$$
\frac{d P}{d t}=\frac{P(i)-P(i-1)}{t(i)-t(i-1)}
$$

When $70 \%$ of the derivatives of the power values are less than the tolerance, $1 \times 10^{-7} \mathrm{~W}$ for linear or $0.1 \mathrm{dBm}$ in $\log$ scale, the measured powers of the laser are considered as stable. After the powers of the laser are considered to be stable, the mean value of the powers is recorded in the spreadsheet as a function of that drive current which is the L-I curve.

The L-I curves are measured at different temperatures. The temperature range is limited 
by the operating temperature of the laser diode and the temperature controlled laser diode mount, ILX Lightwave LDM-4407. The operating temperature of the VCSEL is $-40{ }^{\circ} \mathrm{C}$ to $+85{ }^{\circ} \mathrm{C}$ while the LDM- 4407 temperature controlled laser diode mount is $0{ }^{\circ} \mathrm{C}$ to $70{ }^{\circ} \mathrm{C}$; thus, only the data falling between $0{ }^{\circ} \mathrm{C}$ to $70{ }^{\circ} \mathrm{C}$ are significant. The lower and upper boundary temperatures, $-2{ }^{\circ} \mathrm{C}$ to $73{ }^{\circ} \mathrm{C}$, are set in LabVIEW to control the LDC-3744B that provides the drive current and temperature control to the LDM-4407 and laser diode to ensure all the data are captured within the operating range.

Figure 2-11 shows the threshold current is 1.2mA of the 850nm HFE4192-901 VCSEL at $23{ }^{\circ} \mathrm{C}$ in. There is a small increase in optical power with the drive current below the threshold current. This is noncoherent radiation caused by spontaneous emission in the recombination layer of the laser diode.

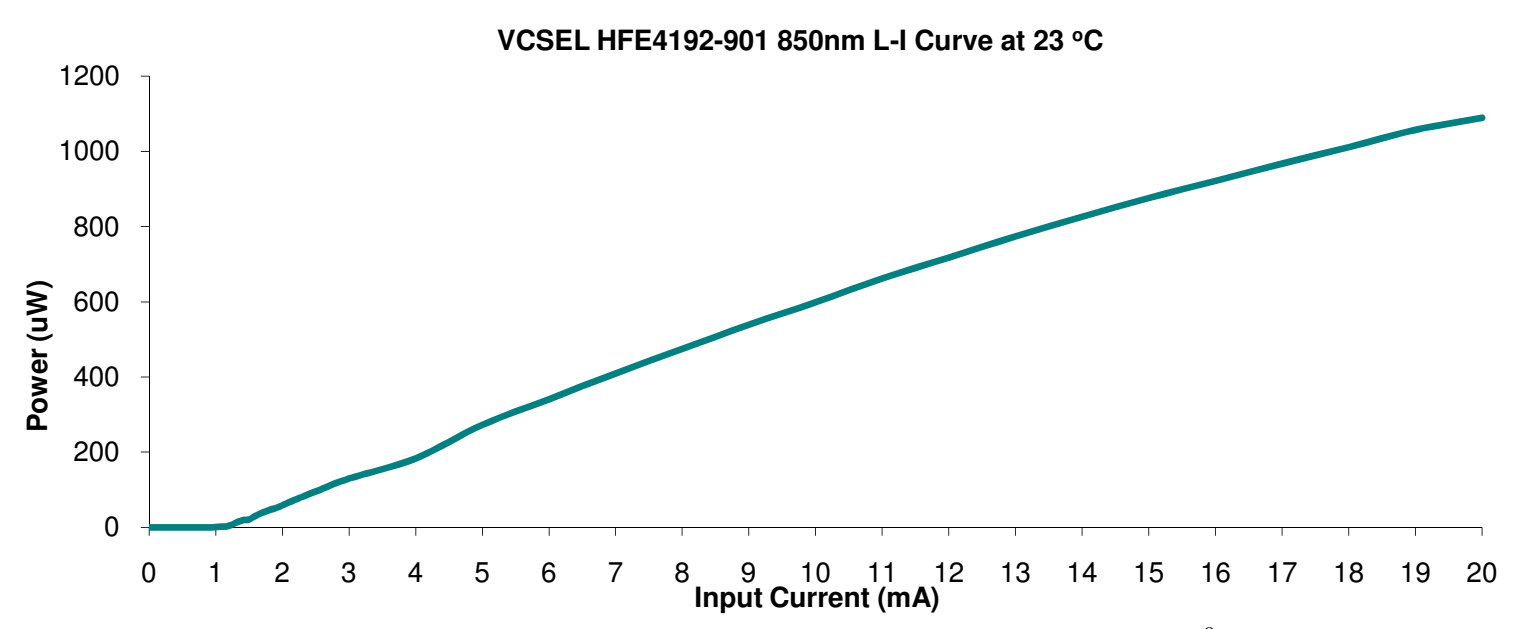

Figure 2-11: L-I curve of the 850nm HFE4192-901 VCSEL at $23{ }^{\circ} \mathrm{C}$

Figure 2-12 shows the L-I curves of the 850nm HFE4192-901 VCSEL from $3{ }^{\circ} \mathrm{C}$ to $68{ }^{\circ} \mathrm{C}$. At a constant current, the laser gain decreases as the temperature rises. This effect can be explained by the nonradiative Auger recombination. It is the dominant contribution to the 
threshold current in which an electron and hole recombine across the band gap and excite a carrier into the valence or conduction band instead of emitting a photon [4]. This interaction normally causes energy to lose in thermal vibration due to free electrons and holes recombine outside the active region, which reduces the number of charges available for optical gain and stimulated emission within the active region. The reduced electrons and holes pairs population within the active region as the temperature rises need more charges or higher drive current to achieve stimulated emission which leads to increase in the threshold currents as shown in Figure 2-13. Figure 2-12 indicates that cooling the laser diode at the same bias input current increases the optical power output.

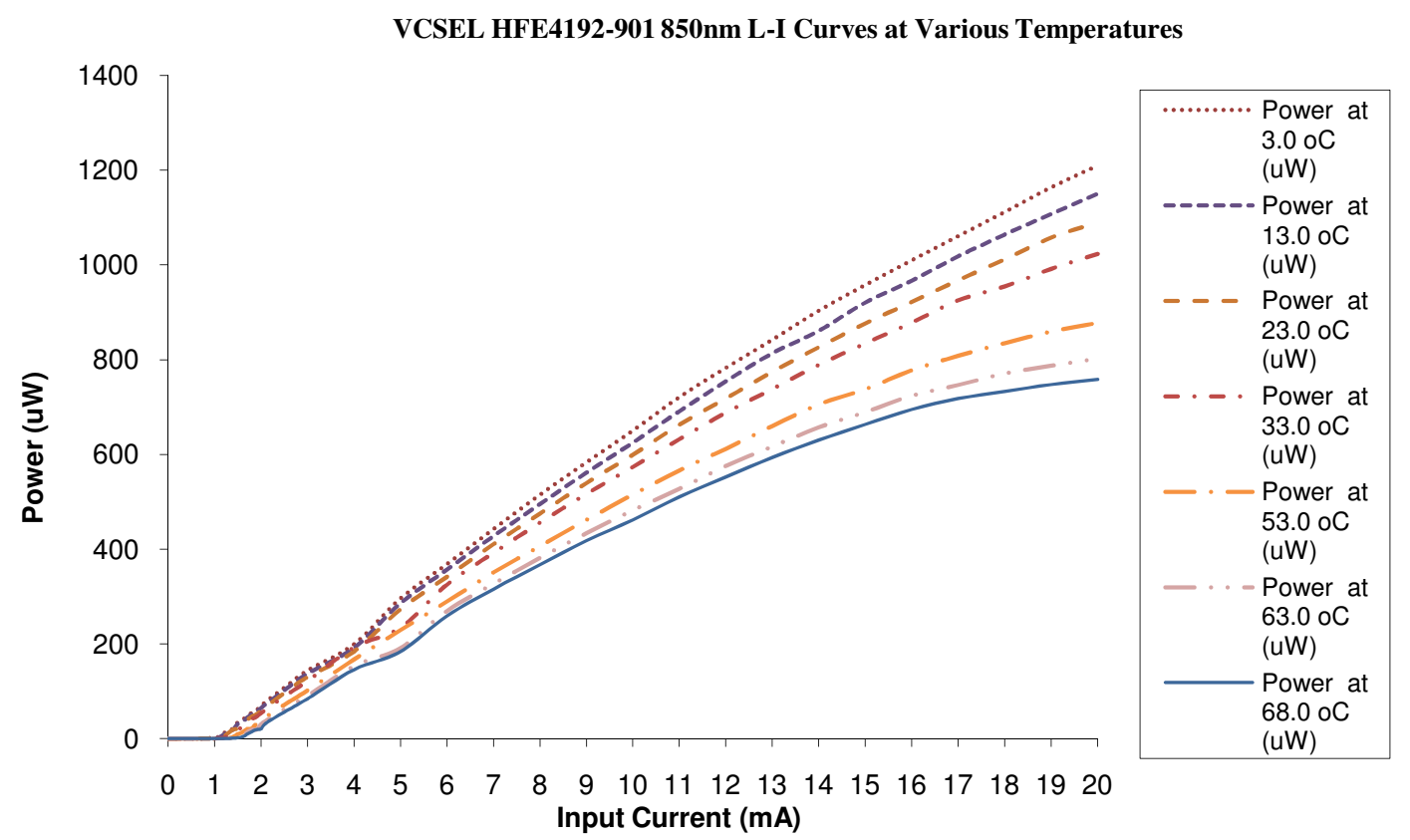

Figure 2-12: L-I curves of the HFE4192-901 VCSEL at various temperatures 


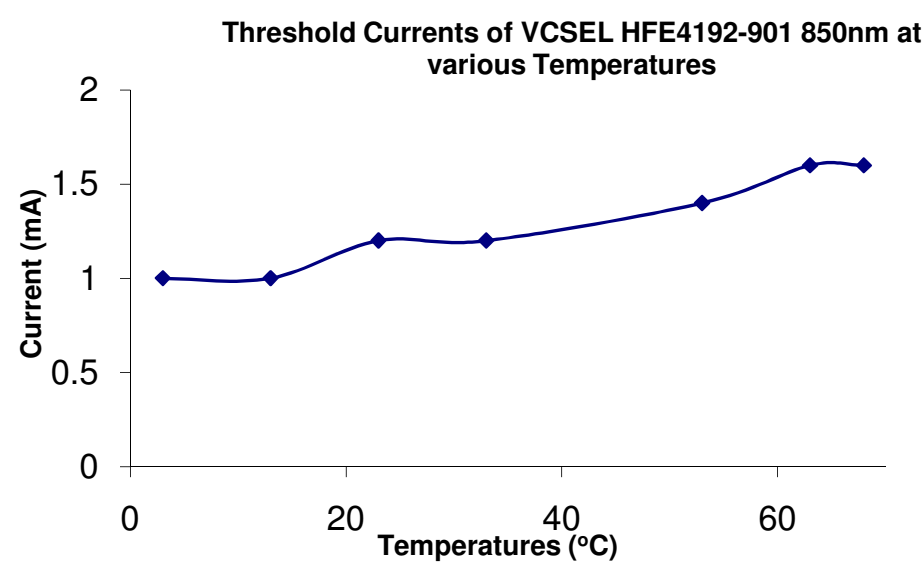

Figure 2-13: Threshold currents of the HFE4192-901 VCSEL at various temperatures

The drive current can also be plotted against the forward biased voltage of the laser diode as shown in Figure 2-14. The voltage is recorded by the LDC-3744B and it is stored into spreadsheet data the LabVIEW code. Similar to the L-I curve, the voltage drops as the temperature rises at a constant input current.

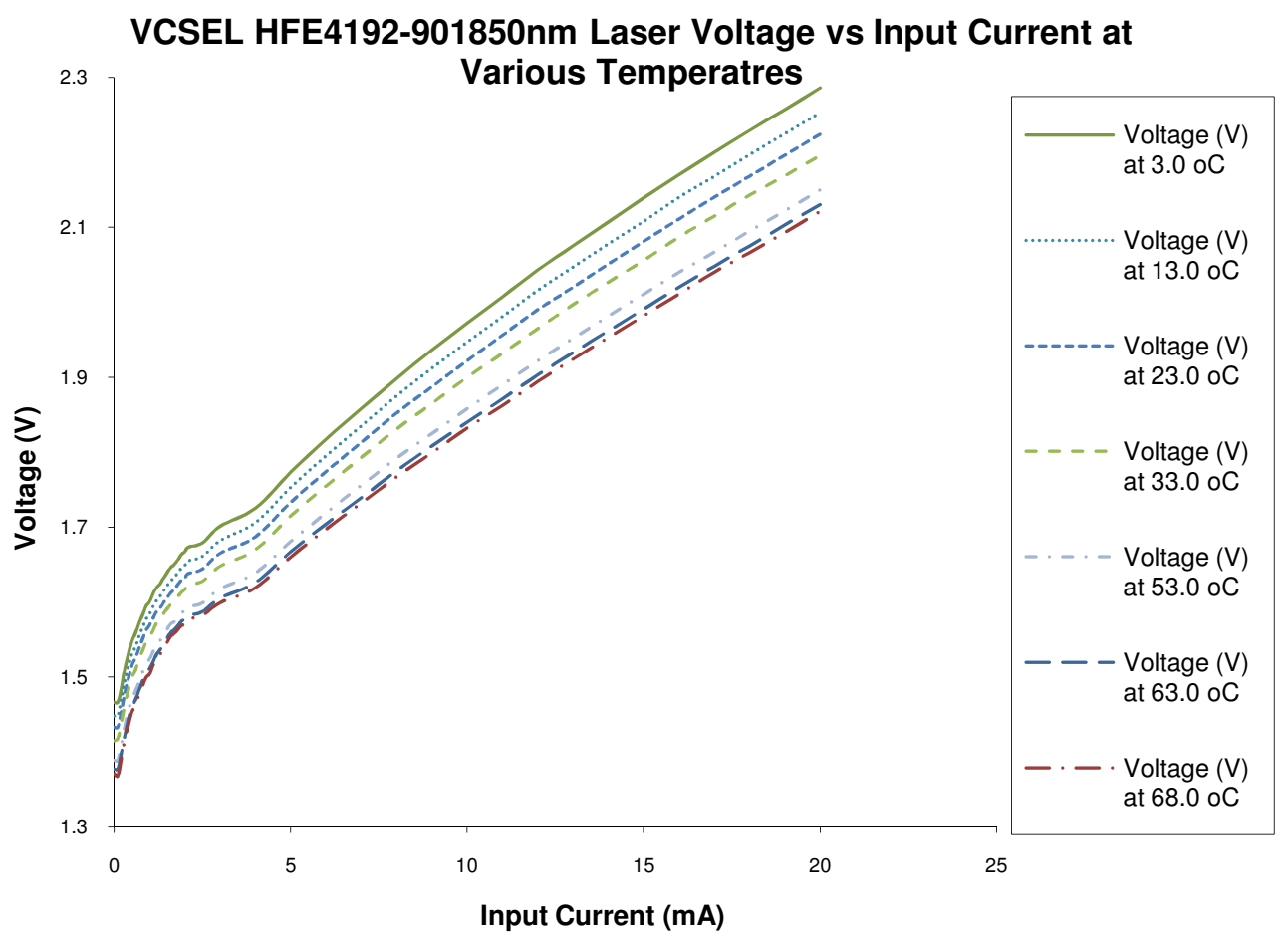

Figure 2-14: VCSEL 850nm Voltages vs. Input Currents at Various Temperatures 


\subsubsection{DFB (Distributed Feedback Laser) L-I Curves}

The 1550nm single mode DFB (Distributed Feedback) laser is controlled by Kette laser diode driver and temperature controller, KNN-2000LT, see Figure 2-15. There are challenges to measure the L-I curve from the DFB laser. First, the KNN-2000LT cannot be automated using LabVIEW and GPIB interface. This is because the operation manual which is supposed to contain the programming codes of the device cannot be found because the manufacturer, Kette, has already defunct. Secondly, there are also no external connectors to connect a digital multi-meter to measure the voltages of the laser. Finally, there is no optical detector for the $1550 \mathrm{~nm}$ range at the time of the experiment. To compensate the challenges, a different measuring technique is used to estimate the L-I curve.

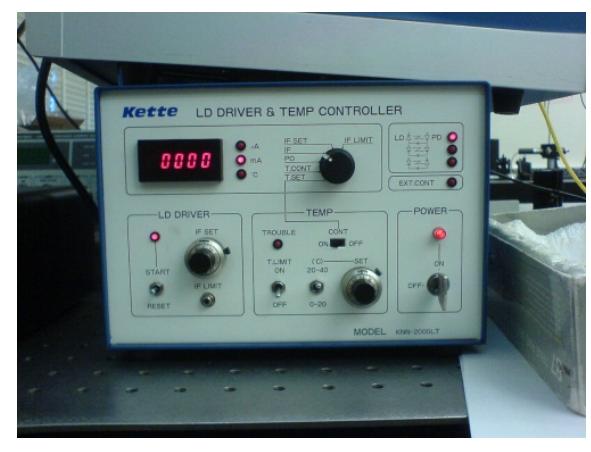

Figure 2-15: Kette Laser Diode Driver and Temperature Controller, KNN-2000LT

A manual approach has to be done to drive the current and control the temperatures of KNN-2000LT for the DFB laser with the absence of LabVIEW and GPIB interface. An optical spectrum analyzer, HP 71450A (see Figure 2-16), connected to the PC via GPIB, is used to measure the total optical power output from the DFB laser. The data is then recorded to the PC that is running the LabVIEW codes. The block diagram of the 
experiment is shown in Figure 2-17.

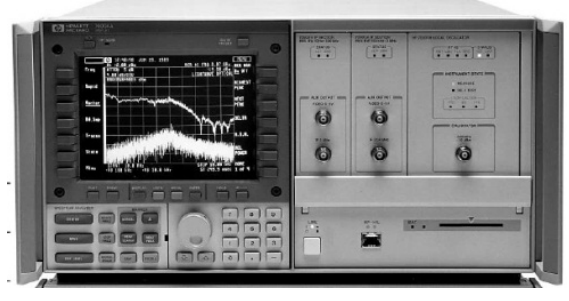

Figure 2-16: HP 71450A Optical Spectrum Analyzer

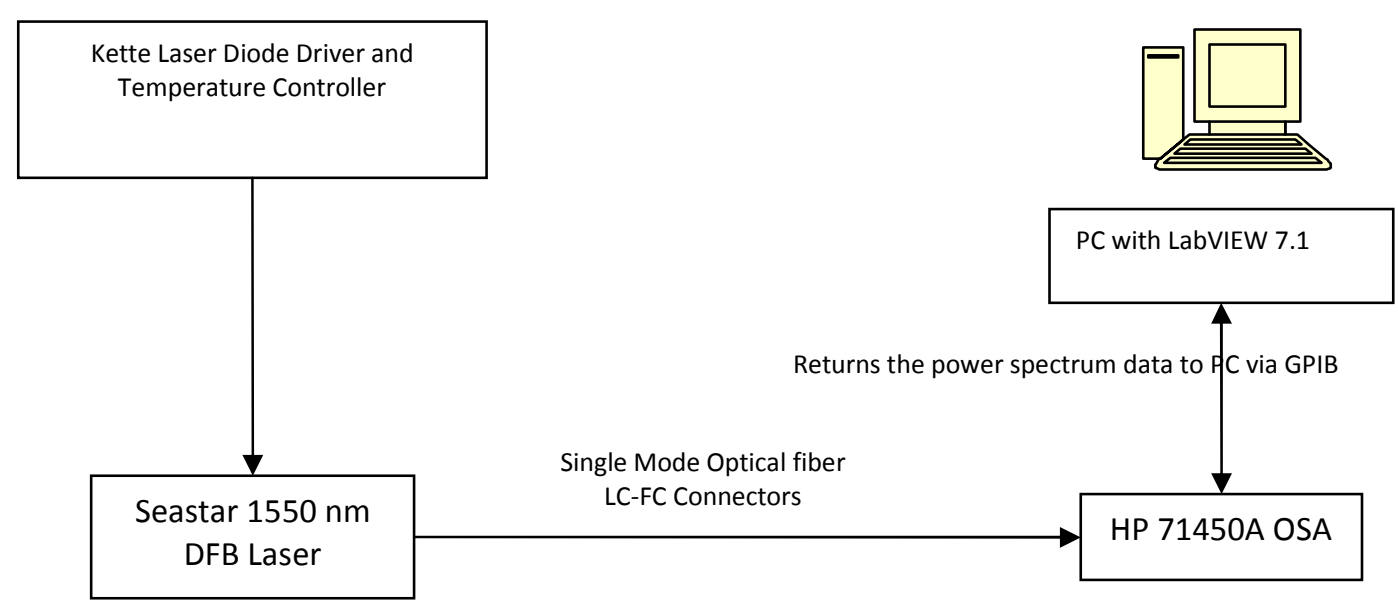

Figure 2-17: Block Diagram of the DFB Laser Optical Power Measurement via OSA

The total optical power, $P_{\text {total }}$, is calculated by taking the integration over the optical power for each point along the optical spectrum and multiplied by the resolution bandwidth [5], RB that is shown in Equation (2.2).

$$
P_{\text {total }}=\frac{R B}{\left(\lambda_{N}-\lambda_{0}\right)} \sum_{i=0}^{N} P(i)
$$

$\mathrm{RB}$ stands for the resolution bandwidth. It is the bandwidth of the IF (Intermediate Frequency) filter, which determines the selectivity of the spectrum analyzer [6]. The unit of resolution bandwidth, $\mathrm{RB}$, is $\mathrm{nm}$, while the power, $P$, is watts. Integrating the product 
respecting to the frequency, $\mathrm{Hz}$, yields the total power in Watts.

A wide resolution bandwidth is required for wide sweeps while a narrow filter is used for narrow sweeps. By using narrower resolution bandwidths, the instrument can reveal sidebands. The penalty for using a higher resolution is a slower sweep speed. Setting 400 instead of 1000 or more data points in the HP 71450A OSA can speed up the data capturing process and to prevent system crash due to insufficient memory in the OSA and the PC that is running the LabVIEW code. However, 400 data points are insufficient to determine the total optical power output, because it is only partial of the optical power. Using the HP 7450A OSA, the optical spectra of the 1550nm DFB laser with drive currents varying from $10 \mathrm{~mA}$ to $30 \mathrm{~mA}$ are recorded at $25^{\circ} \mathrm{C}$, as shown in Figure 2-18.

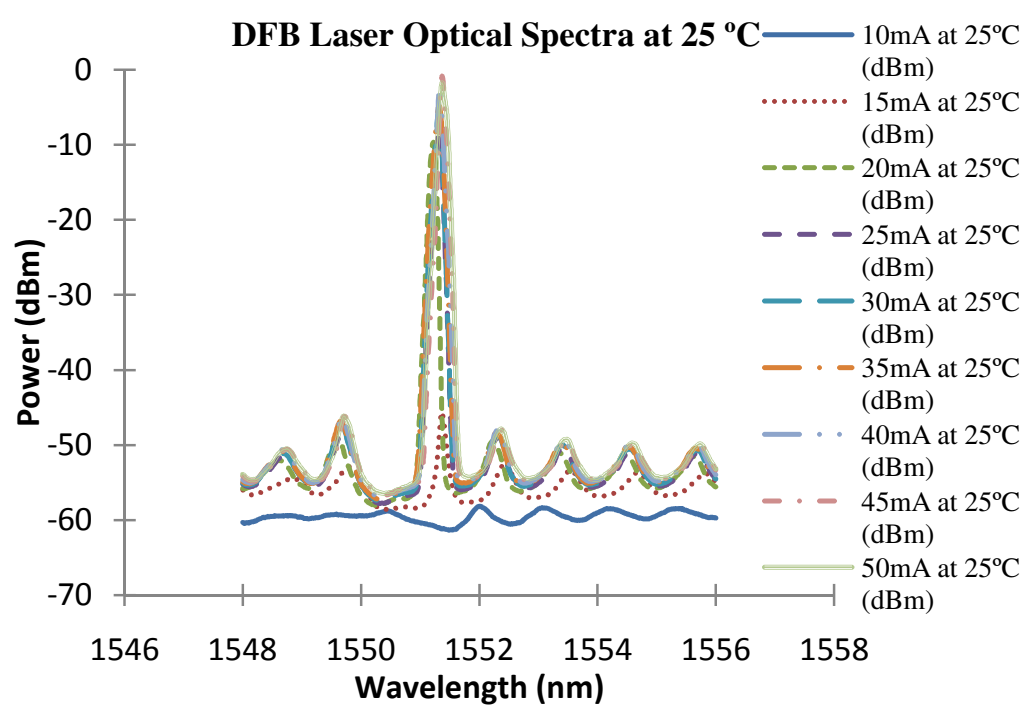

Figure 2-18: DFB Laser Optical Spectra at $25^{\circ} \mathrm{C}$

In Figure 2-19, the threshold current of the L-I curve at $25^{\circ} \mathrm{C}$ is around $16 \mathrm{~mA}$. 


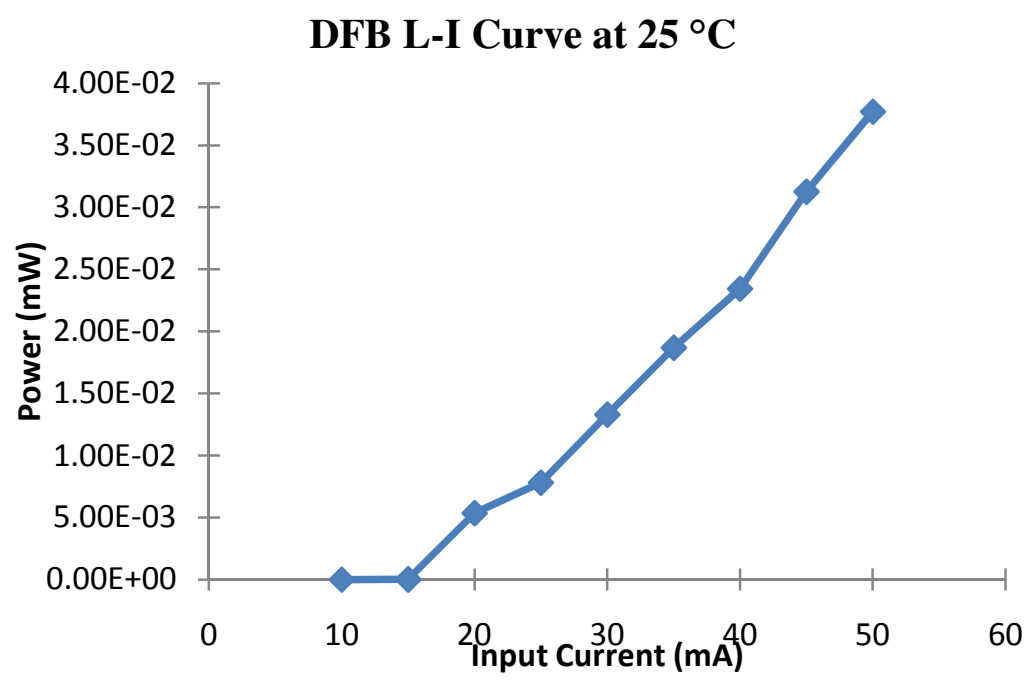

Figure 2-19: DFB L-I Curve at $25^{\circ} \mathrm{C}$ calculated from OSA data

The L-I curves that are plotted along each other at various temperatures from $0{ }^{\circ} \mathrm{C}$ to 35 ${ }^{\circ} \mathrm{C}$ are shown in Figure 2-20.

\section{L-I Curve of DFB Laser at Various Temperatures}

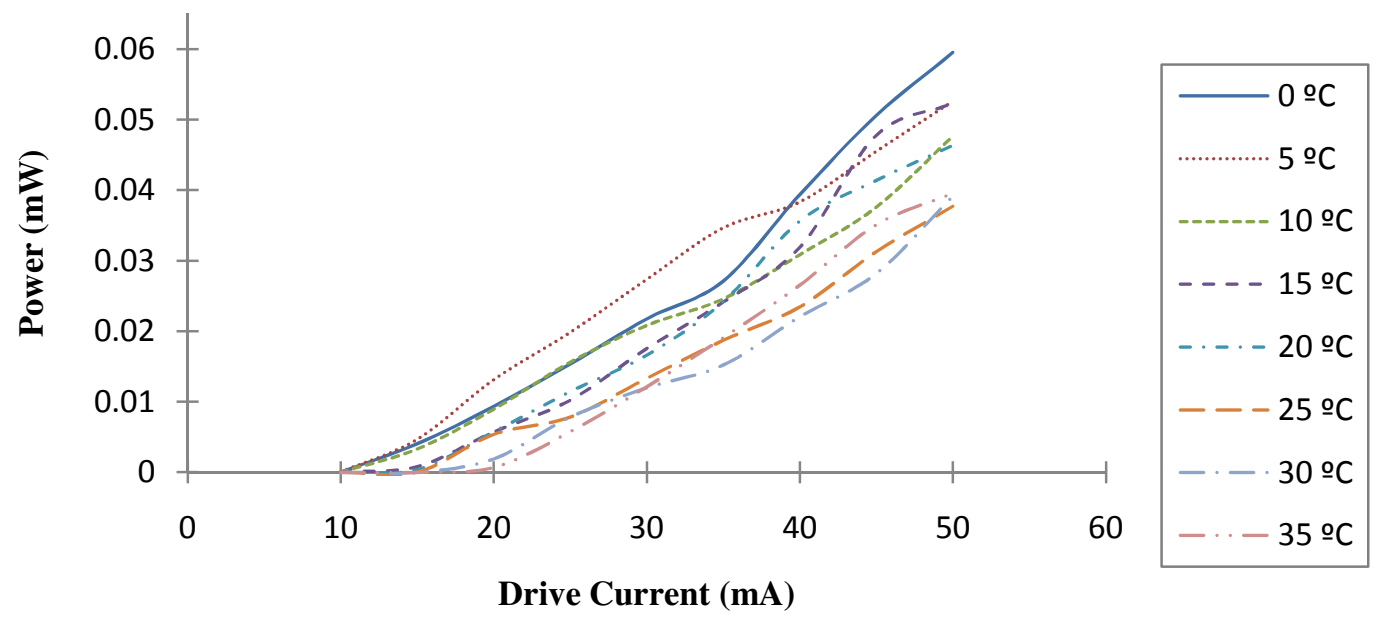

Figure 2-20: DFB Laser L-I Curve at Various Temperatures calculated from OSA data

Notice the L-I curves are fluctuating as the drive current increases and not as consistent as the VCSEL L-I curves from Figure 2-12. The result is partially correct because of taking the integration of the optical spectrum using only limited data points. However, 
similar to the VCSEL L-I curves, the total output optical power of the DFB L-I curves that are calculated by taking the integration of the optical spectrum, drops as the temperature rises with a constant drive current. This is due to the energy loss to thermal vibration where the free electrons and holes recombining outside of the active region without photon emission. Again, the threshold current increases as the temperature rises because it requires more drive current to provide enough electrons to recombine with the holes to increase photon gain in order to achieve stimulated emission.

\subsection{Optical Spectrum Measurement}

\subsubsection{Background in Optical Spectrum Analysis}

The optical spectrum analysis is the measurement of the optical power as a function of wavelength. The applications include testing laser light sources for spectral purity and power distribution as well as testing transmission characteristics of optical devices.

The spectral width of a light source is an important parameter in fiber-optic communication systems due to chromatic dispersion [7], which occurs in the fiber and limits the modulation bandwidth of the system.

Light enters a medium with higher refractive index than vacuum that decreases the velocity of the light wave. The refractive index within the optic fiber varies with wavelength. Therefore, the velocity also varies with wavelength, which is the dispersion. Dispersion causes pulse spread, which distorts the information rate. The pulse spread per unit length can be written as 


$$
\Delta(\tau / L)=-\frac{\lambda}{c} n^{\prime \prime} \Delta \lambda=-M \Delta \lambda
$$

Where $\tau$ is the time it takes for the pulse to travel a path having length $L . \Delta \lambda=\lambda_{2}-\lambda_{1}$ is the spectral width, $M$ is the dispersive material and $n$ " is the $2^{\text {nd }}$ order of index of refraction varies with the wavelength. When $M$ is positive, the pulse spread per unit length is negative. $\Delta(\tau / L)=(\tau / L)_{2}-(\tau / L)_{1}<0,(\tau / L)_{1}>(\tau / L)_{2}$ this means that the traveling time per unit length of the longer wavelength $\lambda_{2},(\tau / L)_{2}$, is faster than that of the shorter wavelength, $\lambda_{1},(\tau / L)_{1}$. When $M$ is negative, the traveling time of the shorter wavelength is faster than the longer wavelength. The information rate depends on the pulse spread per unit length

$$
R \times L=\frac{0.35}{\Delta(\tau / L)}
$$

$R$ stands for data rate in bit/s. From Equation (2.4), when the pulse spread per unit length increases, the data rate decreases. The effect of chromatic dispersion can be seen in the time domain as pulse broadening of a digital waveform. Since chromatic dispersion is a function of the spectral width of the light source, narrow spectral widths are desirable for high-speed communication systems.

\subsubsection{Fabry-Pérot Optical Spectrum Experimental Setup and Results}

The block diagram of setup to measure the optical spectra of the Fabry-Pérot lasers is shown in Figure 2-21. There are four HP Fabry-Pérot laser units at 1300nm being tested in the experiment using the parameters on the HP 71450A OSA as shown in Table 2-1. 
All four FP lasers are single-mode. Since there is only a power supply but no external controller available to change the drive current of the Fabry-Pérot lasers, only the optical power spectra have been tested and recorded to the PC via the LabVIEW automation code for the DC measurements.

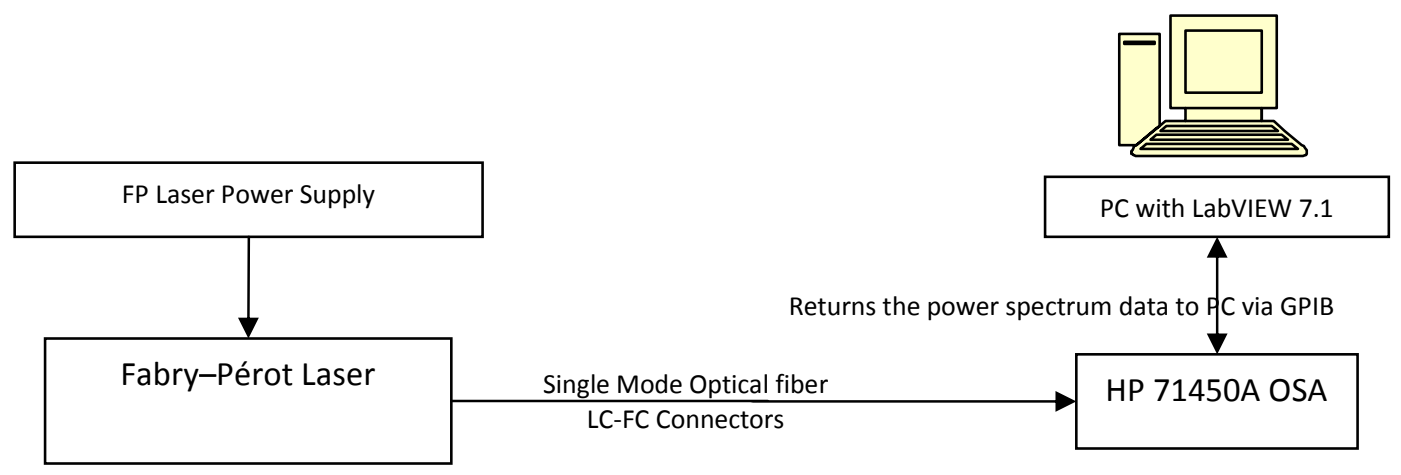

Figure 2-21: Block diagram of Fabry-Pérot optical spectrum experiment setup

Table 2-1: Four HP Fabry-Pérot lasers OSA parameters settings

\begin{tabular}{|r|c|c|c|c|}
\hline & $\begin{array}{c}\# 1 \\
\text { HP3400A }\end{array}$ & $\begin{array}{c}\# 2 \\
\text { HP3400A }\end{array}$ & $\begin{array}{c}\# 3 \\
\text { HP3401A }\end{array}$ & $\begin{array}{c}\# 4 \\
\text { HP3402A }\end{array}$ \\
\hline Resolution Bandwidth (nm): & 0.5 & 0.5 & 0.5 & 0.5 \\
\hline Span (nm): & 50 & 50 & 50 & 40 \\
\hline Sensitivity (dBm): & -52.82 & -52.82 & -52.82 & -52.74 \\
\hline
\end{tabular}

The FP laser resonator region that produces the standing wave patterns only allows integral number of resonant frequencies or longitudinal modes (multi-modes) to exist. The longitudinal modes combining with the gain bandwidth of the laser amplifier, which is a Gaussian curve, produce the optical power spectra that are shown in Figure 2-22a - d. 


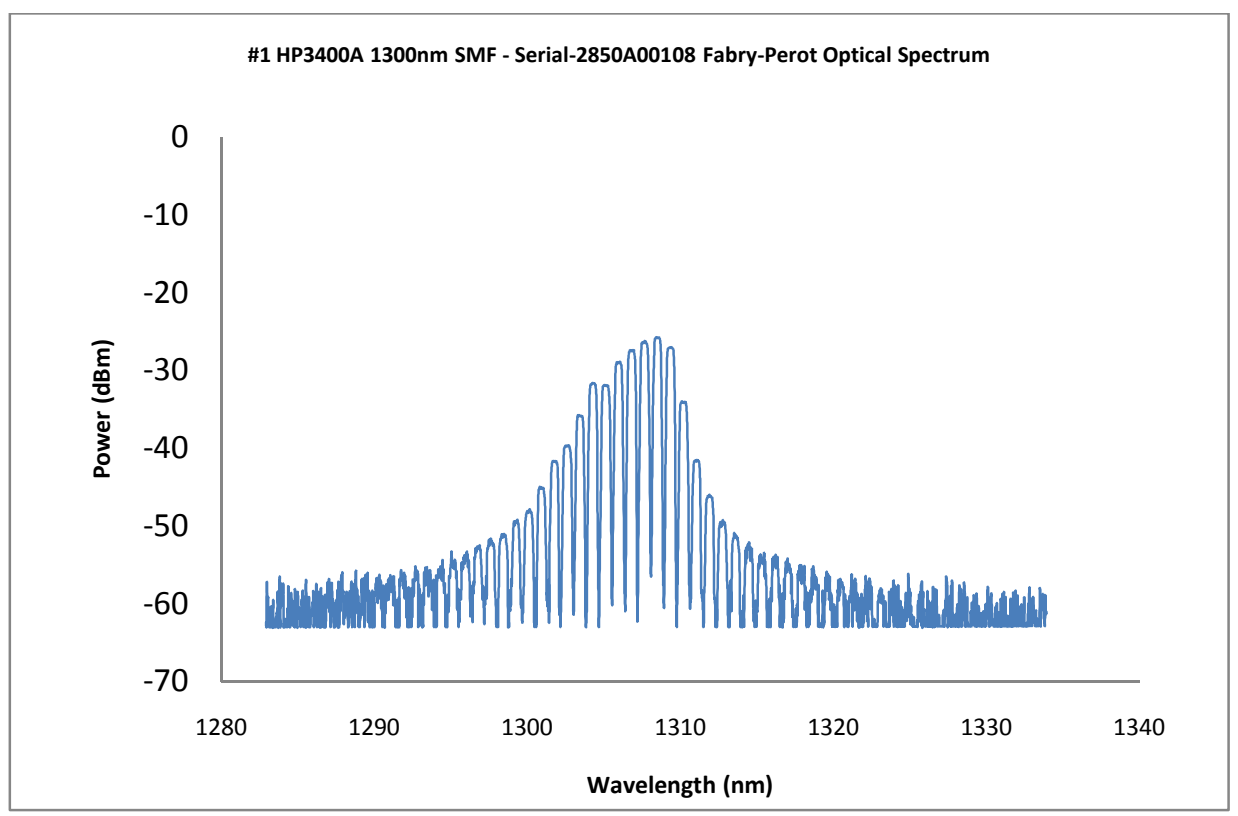

(a)

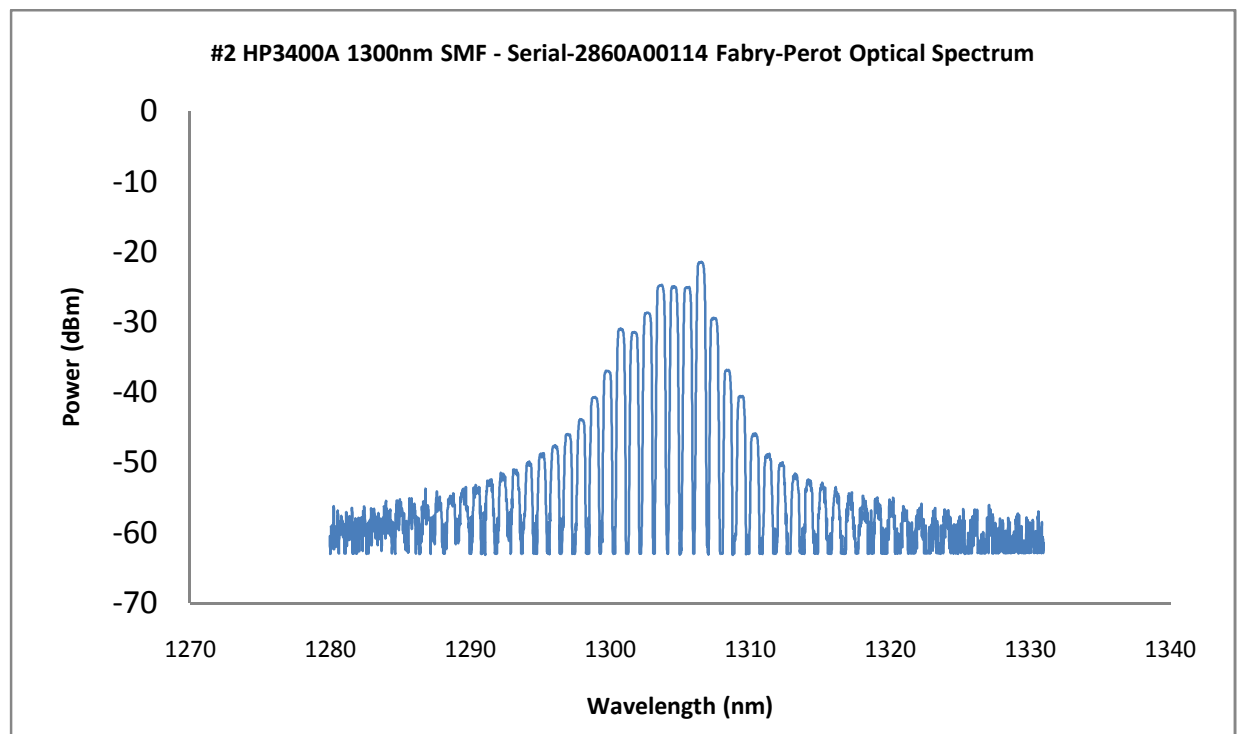

(b)

Figure 2-22 (a), (b): The optical spectra of HP Fabry-Pérot lasers 

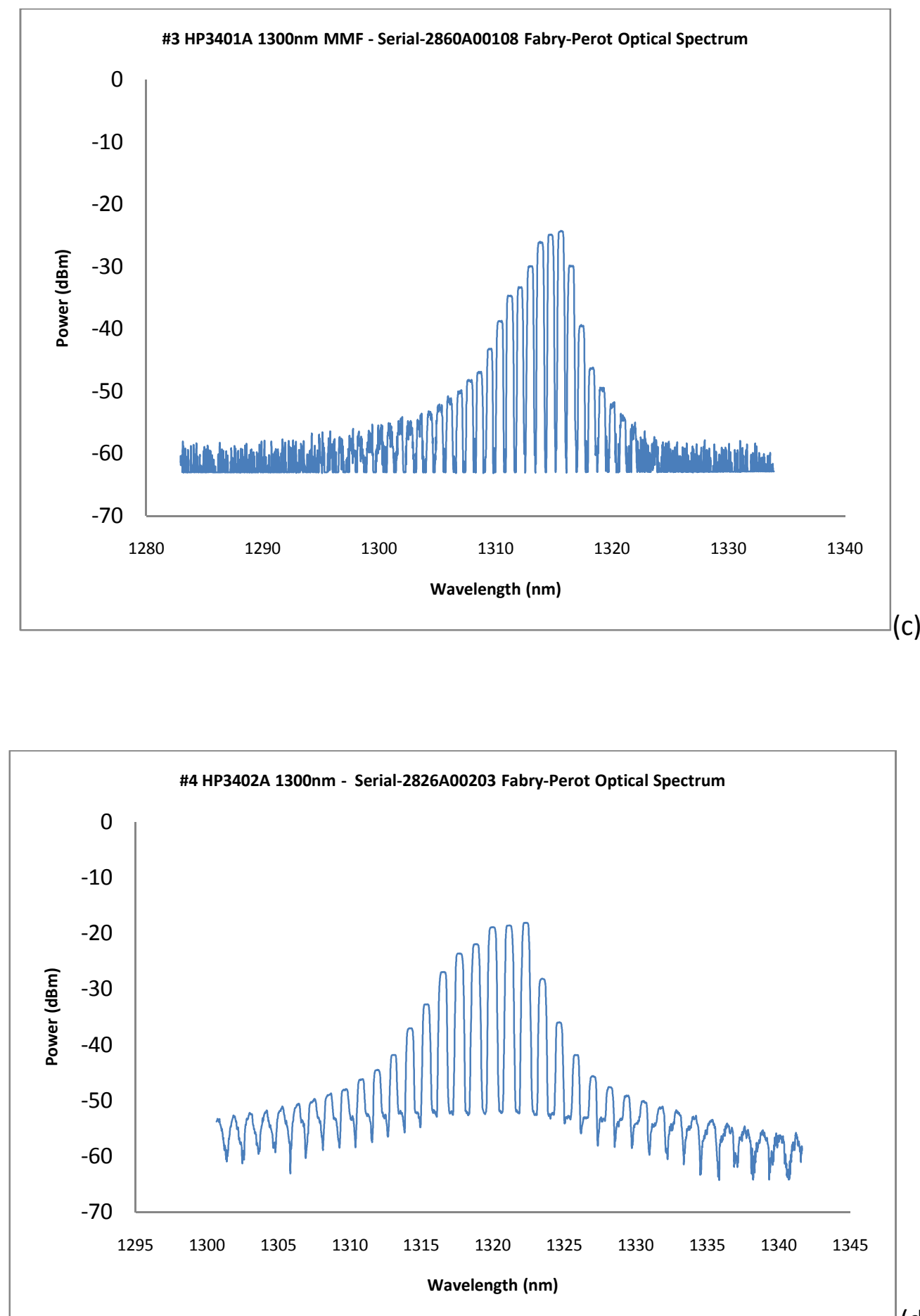

(d)

Figure 2-22 (c), (d): The optical spectra of HP Fabry-Pérot lasers 


\subsubsection{DFB Optical Spectrum Results}

The 1550nm DFB optical spectrum experimental setup in Figure 2-17 is also used for obtaining the L-I curves. The optical spectra that are plotted at $25^{\circ} \mathrm{C}$ with drive currents varying from $10 \mathrm{~mA}$ to $30 \mathrm{~mA}$ are shown in Figure 2-18. As the drive current decreases, the peak of the optical spectrum also decreases. The optical spectra in Figure 2-24 shift at different temperatures, where the drive current is $40 \mathrm{~mA}$ with temperatures starting from $0{ }^{\circ} \mathrm{C}$ to $35^{\circ} \mathrm{C}$ with $5{ }^{\circ} \mathrm{C}$ incrimination for each temperature value. The parameters of the HP 71450A for the DFB OSA measurement are:

\begin{tabular}{|r|c|}
\hline Resolution Bandwidth (nm): & 0.08 \\
\hline Span $(\mathbf{n m}):$ & 10 \\
\hline Sensitivity (dBm): & -80 \\
\hline
\end{tabular}

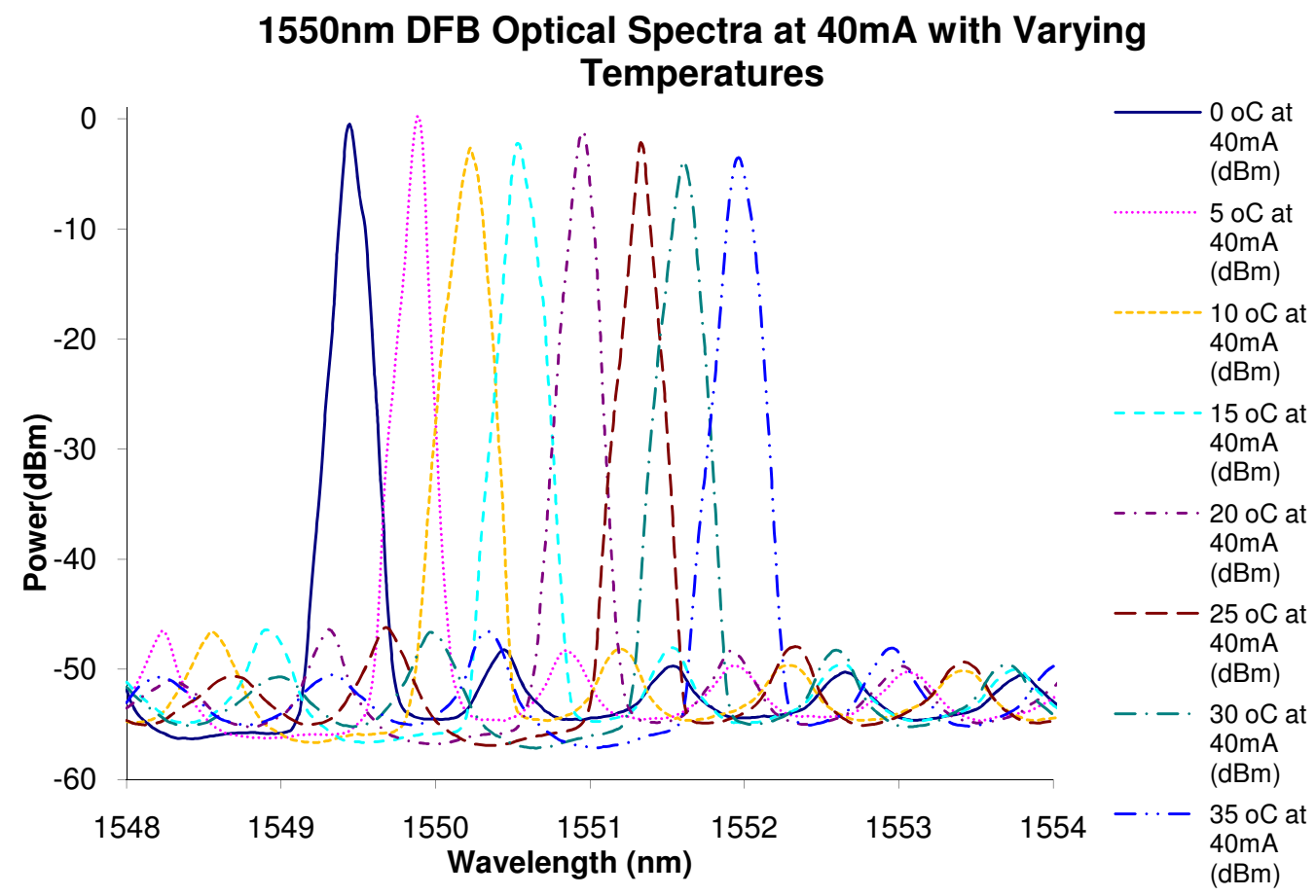

Figure 2-23: DFB OSA at $40 \mathrm{~mA}$ with varying temperatures

The optical spectra of the DFB laser have similar reactions as the VCSEL in Figure 2-23, 
where the center wavelengths increase, shifting from the left to right, as the temperatures increase at a rate of $0.714 \mathrm{~nm} /{ }^{\circ} \mathrm{C}$ with $40 \mathrm{~mA}$ drive current. The wavelength shift phenomenon is similar to what happen in VCSEL, which is also explained in 1.3.2 where the refractive index of the cavity of the laser determines the spacing of the resonant and adjacent resonant wavelengths. The change in temperature varies the guiding layer refractive index, which leads to a shift of the center wavelength and a change to the spacing of the longitudinal modes of a multi-mode laser diode. For a single-mode laser such as the DFB laser, there are several longitudinal modes within the laser's gain, but only one dominates; therefore any temperature change still affects the DFB laser to hop to another longitudinal mode with a different center wavelength [15].

\subsubsection{VCSEL Optical Spectrum}

The experimental setup for VCSEL optical spectrum analysis is shown in Figure 2-24 and the LabVIEW optical spectrum acquisition scheme is mentioned in section 3.2.

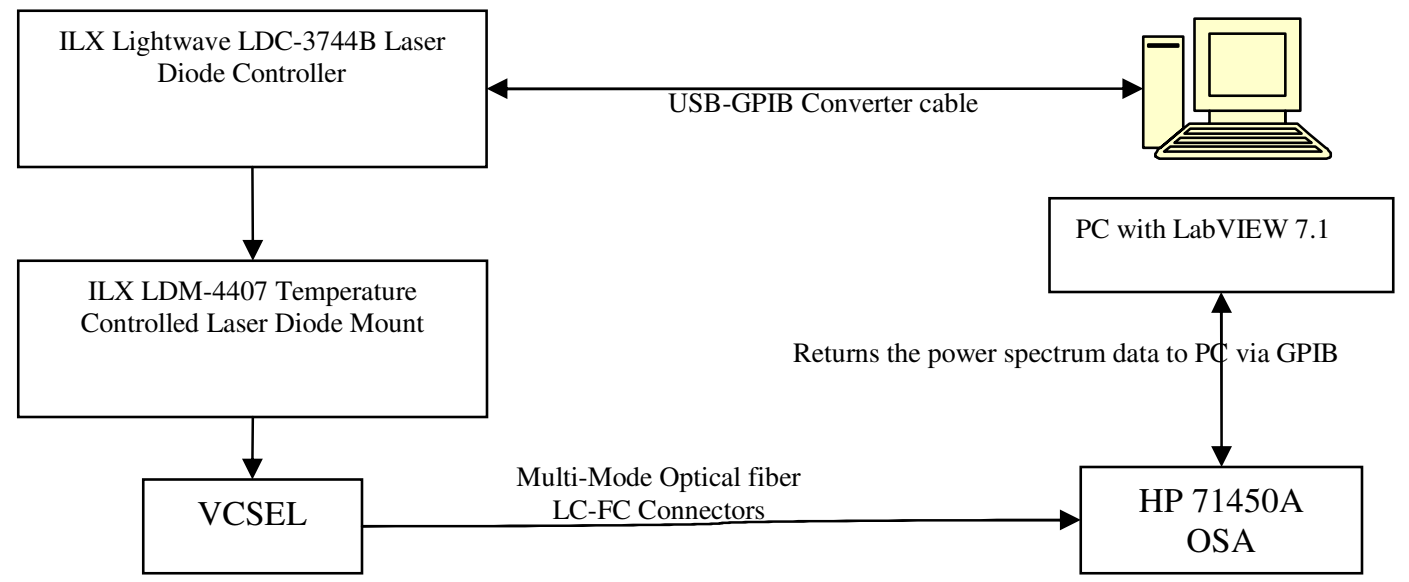

Figure 2-24: Block Diagram of Optical Spectrum Analysis of the 850nm VCSEL 
This setup uses the same laser diode and temperature controller that is controlled by a LabVIEW code in the PC in Figure 2-1 to drive the VCSEL current and change its temperature. The optical spectrum analyzer, HP 71450A, has replaced the power meter and photodetector blocks in Figure 2-1. Two types of optical power spectra are plotted using another LabVIEW code, "Reading indexed data v1.0.vi", from the indexed database files. The script then outputs the optical power spectra into two separate spreadsheets base upon: 1 . a constant temperature at varying drive currents, 2 . a constant drive current at varying temperatures. The details of indexing and reading from the indexed optical power spectrum database are described in Appendix B.

To accurately measure the laser optical power spectrum, the HP 71450A OSA has to be set with the following parameters:

\begin{tabular}{|r|c|}
\hline Resolution Bandwidth (nm): & 0.08 \\
\hline Span (nm): & 10 \\
\hline Sensitivity (dBm): & -75 \\
\hline
\end{tabular}

The span defines the total optical spectrum that is captured by the optical spectrum analyzer. It centers around the center wavelength, $\lambda_{\mathrm{c}}$, so the optical spectrum starts from wavelength $\mathrm{A}, \lambda_{\mathrm{A}}$, to wavelength $\mathrm{B}, \lambda_{\mathrm{B}}$, where

$$
\lambda_{A}=\lambda_{c}-\frac{\operatorname{span}}{2}, \quad \lambda_{B}=\lambda_{c}+\frac{\text { span }}{2}
$$

In this experiment, the VCSEL has a center wavelength at $850 \mathrm{~nm}$, with $10 \mathrm{~nm}$ span. The wavelength starts from $\lambda_{A}$ at $840 \mathrm{~nm}$ to $\lambda_{B} 860 \mathrm{~nm}$. 
The sensitivity is defined as the minimal detectable signal in $\mathrm{dBm}$ or 6 times the rms (root means square) noise level of the optical spectrum analyzer [7]. The sensitivity is set to $-75 \mathrm{dBm}$ for the laser with a center wavelength between $750 \mathrm{~nm}$ to $1100 \mathrm{~nm}$ base on the HP 71450A instruction manual.

\subsubsection{VCSEL Optical Spectrum Results}

The optical spectra of the HFE4192-901 VCSEL at 850nm with a drive current at 16.5mA with varying temperatures that are collected from LabVIEW are plotted.

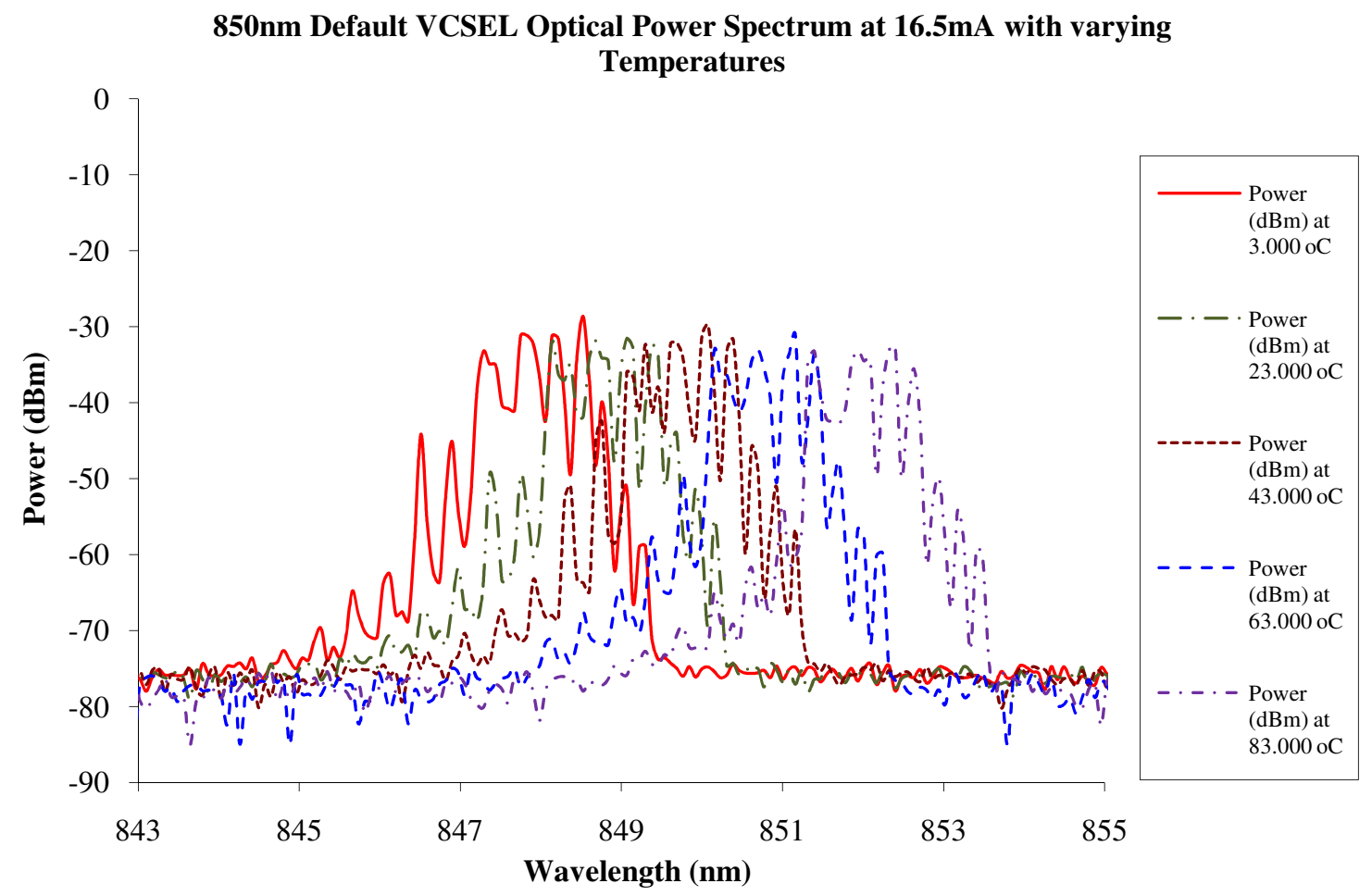

Figure 2-25: VCSEL OSA at 16mA with varying temperatures

The center wavelengths in Figure 2-25 shift from left to right as the temperatures increase. Therefore the center wavelength is directly proportional to the temperature. 
The center wavelength increases approximately $1 \mathrm{~nm}$ for every $20^{\circ} \mathrm{C}$.

The optical spectra that are driven by currents varying from $1 \mathrm{~mA}$ to $19.5 \mathrm{~mA}$ at $23{ }^{\circ} \mathrm{C}$ are shown in Figure 2-26. The optical spectrum peak power increases and shifts to the right towards the longer wavelength as the drive current increases. This is because raising the drive current increases the junction temperature.

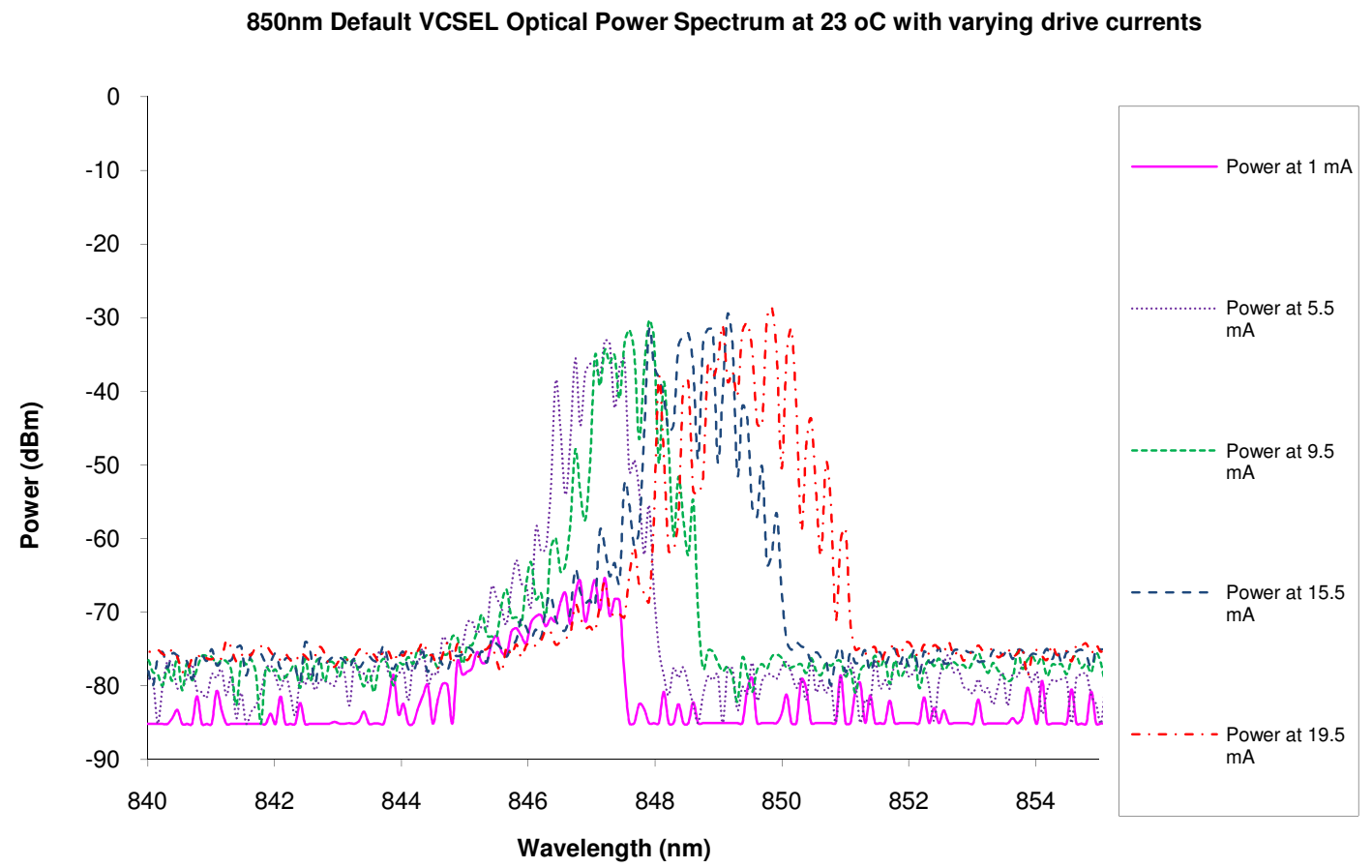

Figure 2-26: VCSEL OSA at $23{ }^{\circ} \mathrm{C}$ with varying drive currents 


\section{Chapter 3: Frequency Response for High Speed}

\section{Measurement of Lasers}

\subsection{Introduction}

Modulation bandwidth describes the optical response of a laser under small sinusoidal changes of bias current. Namely, the transfer function of a laser is significant to modern communications and data transfer systems. The fiber optics communication systems couple the semiconductor laser to one end of the fiber and position the receiver many kilometers away [8]. The laser must have large bandwidth in order to transmit the greatest amount of information. Most laser systems modulate the output laser beam by modulating the bias current to the laser. The ideal case is to have the output beam changes in amplitude by the changes in the bias current to be independent of frequency. However, in real device, the response function usually depends on frequency because electronic circuits have parasitic capacitance causing the frequency to roll-off at high frequencies. The laser output response can be described by the rate equations.

\subsection{Definition of Rate Equations}

The construction of an emitter or detector ensures the interaction between matter and light. Maxwell's equations and the quantum theory describe the details of the interactions, while the rate equations provide the best summary. The rate equations model both the optical and electrical performance of the laser diodes. They describe how the gain, pump, 
feedback, and output coupler mechanisms affect the charge carrier (electrons) and photon concentration in the device. The rate equations manifest the matter-light interaction through the gain term. The gain term represents the photons stimulated emission and absorption which have an incident photon field to operate [8]. The matter-light interaction appears as a gain term that is embedded within the rate equations to describe the most fundamental description of the laser. The rate equations explain the energy conservation in terms of number of excited atoms or the number of carriers in an energy level. They also describe the magnitude and phase of the optical signal.

Semiconductor laser uses electron-hole pair's transition between higher energy level conduction band and lower energy level valence bands to generate light. For the simplest rate equations, we assume an intrinsic semiconductor so that the number of electrons per unit volume (number of electrons $/ \mathrm{cm}^{3}$ ) is equal to the number of holes per unit volume ( $n$ $=p$ ). Since energy is conserved, when an exited electron in the conduction band drops and combines with a hole in the valence band, the energy loss between the two bands is converted into a photon. The change in the photon number has to relate to the number of electron-hole generation and recombination, $N=n V$. The electrons and holes are confined in the active region with volume $V$. The rate equation expresses the difference between the electrons generation and recombination.

$$
\frac{d N}{d t}=\text { Generation }- \text { Recombination }
$$

The electron generation processes include the pump current and absorption which increase the number of electrons in the conduction band. The recombination processes 
include the spontaneous and stimulated emission of photons, without and with an incident photon respectively. When electrons and holes recombine in the valence band, it reduces the total number of electrons in the conduction band. When these factors are incorporated into Equation (3.2.1), the basic rate equation to calculate the change in the number of carriers $n V$ in the active region:

$$
\frac{d N}{d t}=-\left(\begin{array}{c}
\text { Stimulated } \\
\text { Emission }
\end{array}\right)+(\text { Absorption })+\text { Pump }-\left(\begin{array}{c}
\text { Non }- \text { Radiative } \\
\text { Recombination }
\end{array}\right)-\left(\begin{array}{c}
\text { Spontaneous } \\
\text { Recombination }
\end{array}\right)
$$

The pump term describes the number of electron-hole pairs produced in the active volume $V$ per second in the following form:

$$
\text { Pump }=\mathscr{J} V
$$

Where the pump-current density $\mathscr{J}$ has units of $\frac{\# \text { of Carriers }}{\mathrm{Vol} \cdot \mathrm{Sec}}$.

Due to the conservation of energy of matter-light interaction, the processes that decrease the number of carriers $N$ in the conduction band must increase the total number of photons $S=\gamma V_{\gamma}$ in the modal volume $V_{\gamma} \cdot \gamma$ is the photon density in the number of photons per unit volume.

The photon rate equation is written in the following:

$$
\frac{d S}{d t}=+\left(\begin{array}{c}
\text { Stimulated } \\
\text { Emission }
\end{array}\right)-(\text { Absorption })-\left(\begin{array}{c}
\text { Optical } \\
\text { Loss }
\end{array}\right)+\left(\begin{array}{c}
\text { Fraction of } \\
\text { Spontaneous Emission }
\end{array}\right)
$$

The pump current $\mathscr{J}$ in Equation (3.2.2) does not contribute to the photon rate equation in Equation (3.2.4) because it does not directly change the cavity photon number. The optical loss $\alpha_{m}$ in Figure 3-1 includes the lost from the laser cavity when some of the 
light scatters to the side of the cavity sidewalls while some passes through the mirrors, $\mathrm{M}_{1}$ and $\mathrm{M}_{2}$. The light that passes through the mirrors however comprises a useful signal.

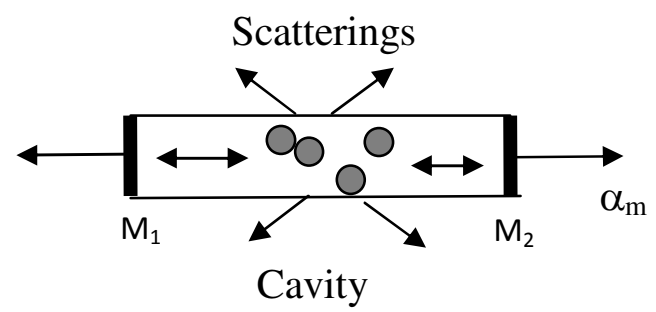

Figure 3-1: Two mechanisms included in the optical loss term in Equation (3.2.4)

\subsection{The General Rate Equations}

The rate Equations (3.2.2) and (3.2.4) are expressed in ordinary differential forms. Dividing Equations (3.2.2) and (3.2.4) by the active volume $\mathrm{V}$ and modal volume $\mathrm{V}_{\gamma}$ respectively and combining the stimulated emission and absorption terms to for the stimulated gain term, the carrier and photon density rate equations are then simplified to the following:

The carrier density rate equation:

$$
\frac{d n}{d t}=-v_{g} g(n) \gamma+J-B n^{2}
$$

The photon density rate equation:

$$
\frac{d \gamma}{d t}=+\Gamma v_{g} g(n) \gamma-\frac{\gamma}{\tau_{\gamma}}+\beta R_{s p}
$$

The pump current density is 


$$
J=\frac{\eta_{i} I}{q V}
$$

in the first carrier density rate Equation (3.3.1) has unit in $\left[\frac{\# \text { of carrier }}{\mathrm{cm}^{3} \cdot \mathrm{sec}}\right] \cdot \eta_{i}$ is the quantum efficiency; $q$ is the charge per electron. The current $I$, is in Ampere where the unit is Coulomb/second. $V$ is the active volume of the laser diode. The electrical current injection increases the number of electrons and holes in the conduction band and valence band respectively. Therefore a positive sign is used in the carrier rate equation.

\subsubsection{Stimulated Emission - Absorption and Gain}

The first term in Equations (3.3.1) and (3.3.2), $v_{g} g(n) \gamma$ is the stimulated emission gain which is a product of three terms: group velocity, material gain, and photon density with an additional term $\Gamma=V / V_{\gamma}$ for the optical confinement factor of the photon density rate equation. The group velocity $v_{g}=c / n_{r}$ where $c$ is the speed of light and $n_{r}$ is index of refraction, has unit length per second $(\mathrm{cm} / \mathrm{s})$. The material gain $g(n)$ has gain in unit length $\left(\right.$ gain/cm), and $\gamma$ is the photon density, \# of photons $/ \mathrm{cm}^{3}$. The unit for the stimulated gain term $v_{g} g(n) \gamma$ is $\left[\frac{\mathrm{cm}}{\mathrm{s}}\right]\left[\frac{\text { gain }}{\mathrm{cm}}\right]\left[\frac{\# \text { of photon }}{\mathrm{cm}^{3}}\right]=\left[\frac{\# \text { of photons }}{\mathrm{cm}^{3} \cdot s}\right]$.

The stimulated emission and absorption affect the number of electrons in conduction band $E_{c}$ and valence band $E_{v}$. This occurs when a photon perturbs the energy levels of atoms and induces radiative recombination. Figure 3-2 (a) shows the stimulated 
recombination. The photon perturbed electron then combines with the hole in the valence band $E_{v}$. The energy-drop from the electron-hole pair is then translated into the form of photon which has the same characteristic as the stimulating photon. Both the stimulating and stimulated photons are in phase therefore coherent. This process is an example of the stimulated emission with the number of electrons decreases while the number of photons increases in the conduction band $E_{c}$.

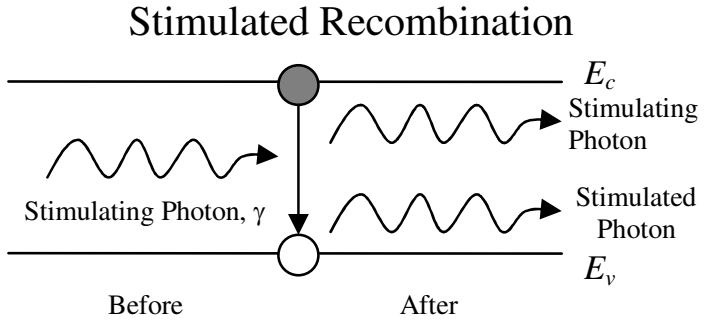

(a)

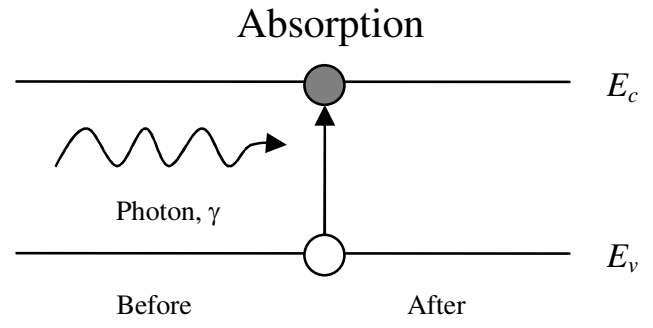

(b)

Figure 3-2: (a) Stimulated recombination and (b) absorption

The opposite condition occurs when an electron in the valence band $E_{v}$ absorbs the energy from the incoming photon. The photon disappears and the electron is excited into the higher energy level in the conduction band $E_{c}$. The process is an example of absorption as shown in Figure 3-2 (b). The stimulated emission increases the number of photons in the laser conduction band while the absorption decreases it. The similar reaction is applied to the number of electrons. Therefore, the gain term should describe the difference between the emission and absorption rates.

Because the stimulated emission process increases and amplifies the photons within the active region in the laser, the photon density is therefore increased while the electron density does the opposite. For carrier density rate Equation (3.3.1), the stimulated 
emission gain term should have a negative sign. The optical confinement factor $\Gamma$ in the stimulated gain term in the photon density rate equation is explained in the following section.

\subsubsection{Optical Confinement Factor}

The physical construction of the semiconductor laser diode is shown in Figure 3-3. The semiconductor to air interfaces to acting as two mirrors on the left and right side of the laser diode. The modal volume $V_{\gamma}$ contains all the optical energy while the active volume $V$ is the laser gain region within the model volume $V_{\gamma}$. The optical power distribution is close to Gaussian shape near the active region (recombination region) which is shown in Figure 3-3. To simplify the laser model, a uniformly distributed optical power is used in the modal volume $V_{\gamma}$, which is represented in the gray area. The optical confinement factor $\Gamma$ defines the fraction of the optical mode that overlaps the gain region $\Gamma=V / V_{\gamma}$.

Therefore the confinement factor is the percentage of the total optical energy found in the active region $V$.

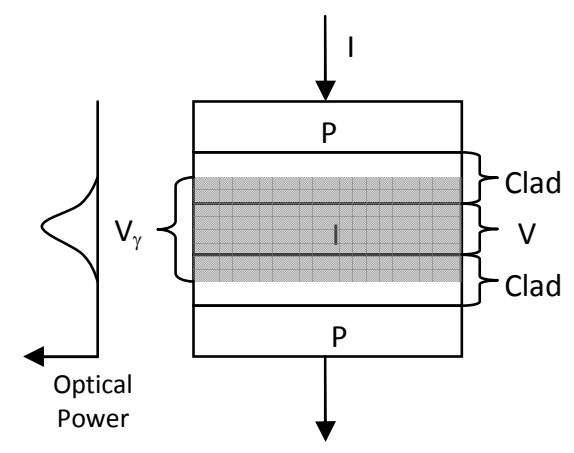

Figure 3-3: Structure of the semiconductor laser 


\subsubsection{Spontaneous Emission Term}

The number of photons increases in the laser from both stimulated and spontaneous emission. The excited atoms in the gain medium (active region) spontaneously emit photons in all directions. The wavelength range of spontaneously emitted photons is very broad. Portions of the spontaneously emitted photons propagate in the same direction as the stimulated photon. Out of these photons that enter the waveguide, only factions of them have the exact frequency to match that of the lasing mode. Therefore, the spontaneous emission term $\beta R_{s p}$ in the photon density rate Equation (3.3.2) is positive and has a geometry factor $\beta$ which describes the fraction of the total spontaneously emitted photons $R_{s p}$ that actually contributes into the lasing mode. $R_{s p}$ has units of number of photons per volume per second.

The last term in the carrier recombination rate Equation (3.3.1) includes both radiative and non-radiative terms. There are three types of laser recombination mechanisms as shown in Figure 3-4 and the total rates of recombination for each type are shown in the following:

- Monomolecular (non-radiative) $A n=\frac{n}{\tau_{n}}$, where $\tau_{n}=1 / A$ represents a lifetime

- Bimolecular $B n^{2}$ (radiative)

- Auger recombination $\mathrm{Cn}^{3}$ (non-radiative)

The total rate of recombination equation is shown below:

$$
R_{r}=R_{\text {radiative }}+R_{\text {non-radiative }}=\frac{n}{\tau_{e}}=A n+B n^{2}+C n^{3}
$$


The units of $R_{r}$ are $\left[\frac{\# \text { of recombination events }}{\mathrm{cm}^{3} \cdot s}\right]$.

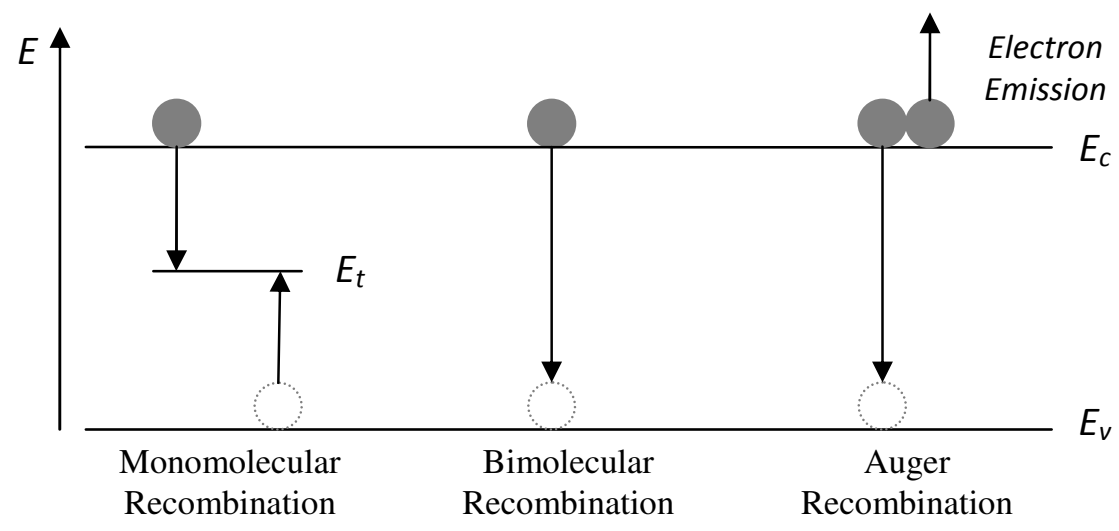

Figure 3-4: Carrier recombination mechanism in semiconductor laser [9]

In most cases, nonradiative recombination occurs primarily through phonon processes. Materials with indirect bandgaps rely on the phonon process for carrier recombination. In Figure 3-4, the monomolecular recombination reduces the laser efficiency by recombining the holes and electrons without emitting photons. This is due to the electrons being trapped in the state $E_{t}$ between the bandgaps and then recombine with the hole in the valence band $E_{v}$. It can also be described as electron and hole being annihilated when they meet together in $E_{t}$.

Bimolecular recombination produces spontaneous emission when an electron from the conduction band $E_{c}$ pairs with the empty valence band $E_{v}$ associated with a hole. The recombination rate is proportional to the product of $p$ and $n$ charges, $n p \rightarrow n^{2}$ where $p=n$. The efficiency is decreased when the energy escapes through the sidewalls of the laser which increases the threshold current, see Figure 3-1. 
Finally, the non-radiative Auger recombination occurs when the energy from the electron-hole pair is transferred to another carrier without emitting a photon. It involves three charged particles (two electrons and a hole) and an energy transfer mechanism; therefore, the recombination rate is proportional to the product of the two electrons and a hole, $n^{2} p \rightarrow n^{3}$ [9]. The carrier lifetime, $\tau_{e}$ is defined below:

$$
\frac{1}{\tau_{e}}=A+B n+C n^{2} \quad \text { and } \quad A=\frac{1}{\tau_{n}}
$$

For lasers that are made with good material, the spontaneous recombination dominates the other recombination processes; therefore the recombination rate can be simplified into:

$$
R_{r}=B n^{2}
$$

Rewrite the total recombination rate in terms of number of carriers and effective carrier lifetime as

$$
\frac{n}{\tau_{e}}=B n^{2}=R_{r}
$$

The carrier density rate equation can be expanded into

$$
\frac{d n}{d t}=-v_{g} g(n) \gamma+\frac{\eta_{i} I}{q V}-\frac{n}{\tau_{e}}
$$

\subsubsection{Optical Loss Term}

The optical loss term describes the changes in the photon density that is related to the optical component of the laser cavity. According to Figure 3-1, the cavity is the space 
bounded by the two mirrors $\mathrm{M}_{1}$ and $\mathrm{M}_{2}$ and sidewalls. Some photons are lost through the sidewalls and mirrors. Some free carriers can absorb light when the light waves drive the motion of the electrons and the surrounding medium from kinetic energy into heat. All these losses can be summarized into the cavity lifetime, $\tau_{\gamma}$. The differential for the optical loss is

$$
\frac{d \gamma}{d t}=-\frac{\gamma}{\tau_{\gamma}}
$$

Solving the differential Equation (3.3.9) $\int \frac{1}{\gamma} d \gamma=-\int \frac{1}{\tau_{\gamma}} d t \rightarrow \ln \gamma=-\frac{t}{\tau_{\gamma}} \rightarrow e^{\ln \gamma}=e^{-\frac{t}{\tau_{\gamma}}}$ yields

$$
\gamma(t)=\gamma_{0} e^{-\frac{t}{\tau_{\gamma}}}
$$

which shows that the initial photon density decays exponentially as the carriers are lost. Equation (3.3.10) describes a lumped device. The amount of energy lost at both mirrors will be averaged over the length of the cavity, which means rate equations depending only on time.

$$
\frac{1}{\tau_{m}}=v_{g} \alpha_{m}=\frac{v_{g}}{L} \ln (1 / R),\left[\frac{1}{s}\right]
$$

$v_{g}=$ group velocity

$\mathrm{L}=$ Length between the mirror

$\mathrm{R}=$ reflectivity

$\alpha_{m}=$ loss of the two mirrors $(1 / \mathrm{cm})$. 
The logarithm term describes the fractional loss at the mirrors. The reciprocal of laser cavity lifetime is the sum of the reciprocals of the internal lifetime $\frac{1}{\tau_{\text {int }}}$ and mirror lifetime $\frac{1}{\tau_{m}}$

The lumped model in Figure 3-5 shows the power of a beam makes a round-trip from $\mathrm{z}=0$ to $\mathrm{z}=\mathrm{L}$ and back to $\mathrm{z}=0$ in the Fabry-Pérot laser cavity. The power is derived from the photon rate Equation (3.3.2) where the spontaneous recombination is ignored.

$$
\frac{d \gamma}{d t}=+\Gamma v_{g} g(n) \gamma-\frac{\gamma}{\tau_{\gamma}}
$$

The distributive losses produce the cavity lifetime of

$$
\frac{1}{\tau_{\gamma}}=\frac{1}{\tau_{\text {int }}}=v_{g} \alpha_{\text {int }}
$$

The mirror-loss term is ignored in Equation (3.3.13) because only the light propagates between the mirrors are considered. Replace the time, $t$ to distance $z$ in Equation (3.3.12) and (3.3.13) and simplify the optical confinement factor $\Gamma=1$ becomes

$$
\frac{d \gamma}{d t}=v_{g} \frac{d \gamma}{d z}=+v_{g} g \gamma-\nu_{g} \alpha_{\text {int }} \quad \rightarrow \quad \frac{d \gamma}{d z}=g \gamma-\gamma \alpha_{\text {int }}=g_{\text {net }} \gamma
$$

The net gain is

$$
g_{\text {net }}=g-\alpha_{\text {int }}
$$

Solve the differential equation of the optical density rate equation in (3.3.14) we have

$$
\frac{d \gamma}{d z}=g_{n e t} \gamma \rightarrow \int \frac{1}{\gamma} d \gamma=\int g_{n e t} d z \rightarrow \ln \gamma=g_{n e t} z \rightarrow \exp (\ln \gamma)=\exp \left(g_{n e t} z\right)
$$




$$
\gamma(z)=\gamma_{0} \exp \left(g_{n e t} z\right)
$$

Since the photon density is proportional to the optical power $P(z)$ traveling through the medium, replace the photon density $\gamma$ in Equation (3.3.16) with $P$ we have

$$
P(z)=P_{0} \exp \left(g_{\text {net }} z\right)
$$

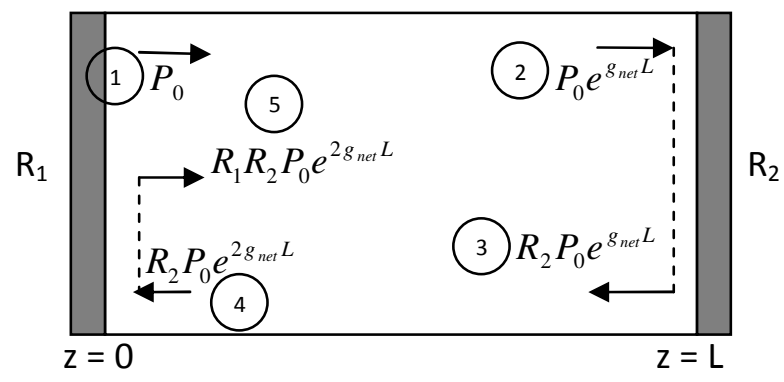

Figure 3-5: The effect of the gain medium on the optical power of a beam making a round-trip in the Fabry-Pérot laser cavity.

Step 1 in Figure 3-5 shows the initial optical power $P_{0}$ at $z=0$ propagates exponentially to $z=\mathrm{L}$ in step 2 just before the right mirror. The optical power is decreased by the mirror 2 reflectance, $\mathrm{R}_{2}$ which results in $R_{2} P_{0} \exp \left(g_{\text {net }} L\right)$ in step 3. The light travels just before hitting mirror 1 at $z=0$ in step 4 with an exponential growth in optical power along the cavity, $R_{2} P_{0} \exp \left(2 g_{n e t} L\right)$. Finally in step 5, the light has completed the roundtrip with the optical power being reduced at the mirror with reflectance $R_{1}$ to $R_{1} R_{2} P_{0} \exp \left(2 g_{n e t} L\right)$. For steady state, the initial power $P_{0}$ must be the same as the final power.

$$
P_{0}=R_{1} R_{2} P_{0} \exp \left(2 g_{n e t} L\right)
$$

Solve for $g_{\text {net }}$ to yield 


$$
g_{\text {net }}=\frac{1}{2 L} \ln \left(\frac{1}{R_{1} R_{2}}\right)=g-\alpha_{\mathrm{int}}
$$

The material gain, $g$ in Equation (3.3.19) can be rewritten in terms of $g_{\text {net }}$ and $\alpha_{\text {int }}$ with reflective coefficient $\Gamma=1$ and $g=\alpha$. Equate Equation (3.3.12) to zero for steady-state and solve for the cavity lifetime, we have the following:

$$
\begin{array}{r}
0=+\Gamma v_{g} g(n) \gamma-\frac{\gamma}{\tau_{\gamma}} \\
\frac{1}{\tau_{\gamma}}=v_{g} g(n)
\end{array}
$$

Assume the two mirrors have the same reflectivity, $R=R_{1}=R_{2}$. Multiply the group velocity $v_{g}$ to both left and right-hand-side to the net gain in Equation (3.3.19), then solve for $v_{g} g(n)$ and finally equate that to Equation (3.3.20) to yield

$$
\frac{1}{\tau_{\gamma}}=v_{g} \alpha_{\text {int }}+\frac{v_{g}}{L} \ln \left(\frac{1}{R}\right)
$$

Equation (3.3.21) shows the lifetime of the light energy escapes from the laser cavity through the internal and mirrors losses.

\subsection{Modulation Bandwidth}

\subsubsection{Small Signal Analysis}

Most laser systems modulate the output laser beam by modulating over the current to the laser. The DC current is around the threshold current, where the stimulated emission begins, as the binary 0 . The binary 1 is generated when the current signal has a positive 
pulse. When the biased current is near the threshold, the laser diode will turn on more quickly. Just like any electronic circuit, the laser diode has its limit to keep up with the gain at high frequencies. Therefore, the rate equations are used to derive the transfer function of the laser diode to find the frequency response and the bandwidth. For simplicity, the laser gain is linearized and shown below using Taylor Expansion. The prime of the gain $n^{\prime}$ denotes the total of the DC and AC quantities. The $n^{\prime}$ is the total DC and $\mathrm{AC}$ carrier density value and $n_{0}$ is the carrier density DC value.

$$
g\left(n^{\prime}\right)=\sum_{k=0}^{\infty} \frac{g^{(k)}\left(n_{0}\right)}{k !}\left(n^{\prime}-n_{0}\right)^{k}=g\left(n_{0}\right)+g^{\prime}\left(n_{0}\right)\left(n^{\prime}-n_{0}\right)
$$

The gain is further simplified to: $g\left(n^{\prime}\right)=g_{0}\left(n^{\prime}-n_{0}\right)$, where the laser gain $g_{0}=g\left(n_{0}\right)$ and the laser differential gain $g^{\prime}=\partial g / \partial n$.

The rate equations in total AC and DC quantities are shown below. The recombination term is linearized by using $n^{\prime} / \tau_{n}$ in the carrier density rate equation. The spontaneous recombination term in the photo density rate is eliminated because the density rate is dominated by the stimulated gain.

The carrier density rate equation:

$$
\frac{d n^{\prime}}{d t}=-v_{g} g_{0}\left(n^{\prime}-n_{0}\right) \gamma^{\prime}+J^{\prime}-\frac{n^{\prime}}{\tau_{n}}
$$

The photon density rate equation:

$$
\frac{d \gamma^{\prime}}{d t}=+\Gamma v_{g} g_{0}\left(n^{\prime}-n_{0}\right) \gamma^{\prime}-\frac{\gamma^{\prime}}{\tau_{\gamma}}
$$

The flow chart to derive the rate equations into transfer function is shown in Figure 3-6. 


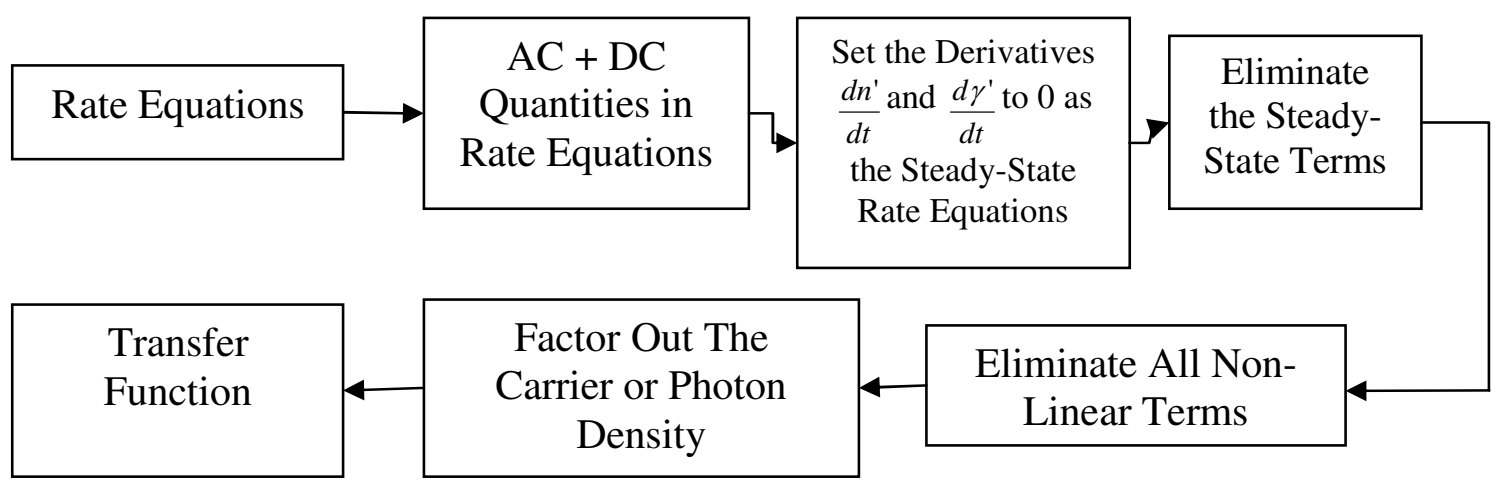

Figure 3-6: Flow Chart of Derivation of the Transfer Function from Rate Equations

The primed quantities are defined as the total AC and DC terms:

$$
n^{\prime}=\bar{n}+n e^{j \omega t} \quad \gamma^{\prime}=\bar{\gamma}+\gamma e^{j \omega t} \quad J^{\prime}=\bar{J}+J e^{j \omega t}
$$

The steady-state DC quantities are denoted by a bar over the quantity such as $\bar{n}$ which also means the average. The sinusoidal AC quantities are denoted by the Euler's formula $e^{j \omega t}$. In small signal analysis, when considering the steady-state quantities only, the derivatives of the carrier and photon densities rate equations have to be set to zero and also eliminate the $\mathrm{AC}$ signals $e^{j \omega t}$.

$$
\begin{aligned}
& 0=-v_{g} g_{0}\left(\bar{n}-n_{0}\right) \bar{\gamma}+\bar{J}-\frac{\overline{\mathrm{n}}}{\tau_{\mathrm{n}}} \\
& 0=+\Gamma v_{g} g_{0}\left(\bar{n}-n_{0}\right) \bar{\gamma}-\frac{\bar{\gamma}}{\tau_{\gamma}}
\end{aligned}
$$

Define $\bar{g}=g_{0}\left(\bar{n}-n_{0}\right)$ and substitute (3.4.4) into Equation (3.4.2) and (3.4.3) to have

$$
\begin{aligned}
& j \omega n e^{j \omega t}=-v_{g}\left(\bar{g}+g_{0} n e^{j \omega t}\right)\left(\bar{\gamma}+\gamma e^{j \omega t}\right)+\bar{J}-\frac{\bar{n}}{\tau_{n}}+J e^{j \omega t}-\frac{n}{\tau_{n}} e^{j \omega t} \\
& j \omega \gamma e^{j \omega t}=+\Gamma v_{g}\left(\bar{g}+g_{0} n e^{j \omega t}\right)\left(\bar{\gamma}+\gamma e^{j \omega t}\right)-\frac{\bar{\gamma}}{\tau_{\gamma}}-\frac{\gamma e^{j \omega t}}{\tau_{\gamma}}
\end{aligned}
$$


Subtract the steady-state terms, equations (3.4.5) and (3.4.6), from Equation (3.4.7) and (3.4.8) respectively to obtain the following:

Carrier Density:

$$
\begin{aligned}
j \omega n e^{j \omega t} & =-v_{g}\left(\bar{g}+g_{0} n e^{j \omega t}\right)\left(\bar{\gamma}+\gamma e^{j \omega t}\right)+\bar{J}-\frac{\bar{n}}{\tau_{n}}+J e^{j \omega t}-\frac{n}{\tau_{n}} e^{j \omega t}+v_{g} \bar{g} \bar{\gamma}-\bar{J}+\frac{\bar{n}}{\tau_{\mathrm{n}}} \\
& =-v_{g} \bar{g} \gamma e^{j \omega t}-v_{g} g_{0} n e^{j \omega t} \bar{\gamma}-v_{g} g_{0} n \gamma e^{2 j \omega t}+J e^{j \omega t}-\frac{n}{\tau_{n}} e^{j \omega t}
\end{aligned}
$$

Photon Density:

$$
\begin{aligned}
j \omega \gamma e^{j \omega t} & =+\Gamma v_{g}\left(\bar{g}+g_{0} n e^{j \omega t}\right)\left(\bar{\gamma}+\gamma e^{j \omega t}\right)-\frac{\bar{\gamma}}{\tau_{\gamma}}-\frac{\gamma e^{j \omega t}}{\tau_{\gamma}}-\Gamma v_{g} \bar{g} \bar{\gamma}+\frac{\bar{\gamma}}{\tau_{\gamma}} \\
& =+\Gamma v_{g} \bar{g} \gamma e^{j \omega t}+\Gamma v_{g} g_{0} n e^{j \omega t} \bar{\gamma}+\Gamma v_{g} g_{0} n \gamma e^{2 j \omega t}-\frac{\gamma e^{j \omega t}}{\tau_{\gamma}}
\end{aligned}
$$

Drop all the second-order nonlinear terms such as $m, n^{2}$, and $e^{2 j \omega t}$. Finally cancel the $e^{j \omega t}$ terms in Equation (3.4.9) and (3.4.10) to get

$$
\begin{aligned}
& j \omega n=-v_{g} \bar{g} \gamma-v_{g} g_{0} n \bar{\gamma}+J-\frac{n}{\tau_{n}} \\
& j \omega \gamma=+\Gamma v_{g} \bar{g} \gamma+\Gamma v_{g} g_{0} n \bar{\gamma}-\frac{\gamma}{\tau_{\gamma}}
\end{aligned}
$$

Solve for the photon density $\gamma$ and carrier density $n$ from the carrier density and photon density rate equations in Equation (3.4.11) and (3.4.12). Let $\alpha=\left(v_{g} g_{0} \bar{\gamma}+\frac{1}{\tau_{n}}+j \omega\right)$ and $\beta=\left(\frac{1}{\tau_{\gamma}}-\Gamma v_{g} \bar{g}+j \omega\right)$ 


$$
\begin{aligned}
& \gamma=\frac{J-\left(v_{g} g_{0} \bar{\gamma}+\frac{1}{\tau_{n}}+j \omega\right) n}{v_{g} \bar{g}}=\frac{J-\alpha n}{v_{g} \bar{g}} \\
& n=\frac{\left(\frac{1}{\tau_{\gamma}}-\Gamma v_{g} \bar{g}+j \omega\right) \gamma}{\Gamma v_{g} g_{0} \bar{\gamma}}=\frac{\beta \gamma}{\Gamma v_{g} g_{0} \bar{\gamma}}
\end{aligned}
$$

Substitute (3.4.14) into (3.4.13) to obtain the photon density below

$$
\gamma=\frac{J-\alpha\left(\frac{\beta \gamma}{\Gamma v_{g} g_{0} \bar{\gamma}}\right)}{v_{g} \bar{g}}
$$

The step-by-step procedures to solve for the photon density are shown below:

$$
\begin{aligned}
& v_{g} \bar{g} \gamma=J-\alpha\left(\frac{\beta \gamma}{\Gamma v_{g} g_{0} \bar{\gamma}}\right) \\
& \left(v_{g} \bar{g}+\frac{\alpha \beta}{\Gamma v_{g} g_{0} \bar{\gamma}}\right) \gamma=J \\
& \left(\frac{\Gamma v_{g}^{2} g_{0} \bar{\gamma} \bar{g}+\alpha \beta}{\Gamma v_{g} g_{0} \bar{\gamma}}\right) \gamma=J
\end{aligned}
$$

Solve for the transfer function with electrons as input and photons as output $\frac{\gamma}{J}$ :

$$
\frac{\gamma}{J}=\frac{\Gamma v_{g} g_{0} \bar{\gamma}}{\Gamma v_{g}^{2} g_{0} \bar{\gamma} \bar{g}+\alpha \beta}
$$

Substitute $\alpha$ and $\beta$ in back to (3.4.16) and solve for the transfer function,

$$
\frac{\gamma}{J}=\frac{\Gamma v_{g} g_{0} \bar{\gamma}}{\Gamma v_{g}^{2} g_{0} \bar{\gamma} \bar{g}+\left(v_{g} g_{0} \bar{\gamma}+\frac{1}{\tau_{n}}+j \omega\right)\left(\frac{1}{\tau_{\gamma}}-\Gamma v_{g} \bar{g}+j \omega\right)}
$$


Expand the denominator of the transfer function

$$
\begin{aligned}
& D=\Gamma v_{g}^{2} g_{0} \bar{\gamma} \bar{g}+\left(v_{g} g_{0} \bar{\gamma}+\frac{1}{\tau_{n}}+j \omega\right)\left(\frac{1}{\tau_{\gamma}}-\Gamma v_{g} \bar{g}+j \omega\right) \\
& =\Gamma v_{g}^{2} g_{0} \bar{\gamma} \bar{g}+\left(v_{g} g_{0} \bar{\gamma}+\frac{1}{\tau_{n}}\right)\left(\frac{1}{\tau_{\gamma}}-\Gamma v_{g} \bar{g}\right)-\omega^{2}+j \omega\left(v_{g} g_{0} \bar{\gamma}+\frac{1}{\tau_{n}}+\frac{1}{\tau_{\gamma}}-\Gamma v_{g} \bar{g}\right) \\
& \text { Define the terms }\left\{\begin{array}{l}
\omega_{0}^{2}=\Gamma v_{g}^{2} g_{0} \bar{\gamma} \bar{g}+\left(v_{g} g_{0} \bar{\gamma}+\frac{1}{\tau_{n}}\right)\left(\frac{1}{\tau_{\gamma}}-\Gamma v_{g} \bar{g}\right) \\
\frac{1}{\tau}=\left(v_{g} g_{0} \bar{\gamma}+\frac{1}{\tau_{n}}+\frac{1}{\tau_{\gamma}}-\Gamma v_{g} \bar{g}\right)
\end{array}\right.
\end{aligned}
$$

Substitute the fundamental frequency $\omega_{0}^{2}$ and life time $\frac{1}{\tau}$ into (3.4.18) to obtain the denominator of the transfer function.

$$
D=\left(\omega_{0}^{2}-\omega^{2}\right)+\frac{j \omega}{\tau}
$$

The transfer function becomes

$$
\frac{\gamma}{J}=\frac{\Gamma v_{g} g_{0} \bar{\gamma}}{D}=\frac{\Gamma v_{g} g_{0} \bar{\gamma}}{\left(\omega_{0}^{2}-\omega^{2}\right)+\frac{j \omega}{\tau}}
$$

Find response function by taking the magnitude of the transfer function.

$$
R_{f}=\left|\frac{\gamma}{J}\right|^{2}=\frac{\left(\Gamma v_{g} g_{0} \bar{\gamma}\right)^{2}}{\left(\omega_{0}^{2}-\omega^{2}\right)^{2}+\left(\frac{\omega}{\tau}\right)^{2}}
$$

Take the derivative of the response function and equate that to zero, $\frac{d R_{f}}{d \omega}=0$ to obtain the resonant frequency $\omega_{r}^{2}$. 


$$
\begin{gathered}
\frac{d R_{f}}{d \omega}=\frac{d}{d \omega}\left(\frac{\left(\Gamma v_{g} g_{0} \bar{\gamma}\right)^{2}}{\left(\omega_{0}^{2}-\omega^{2}\right)^{2}+\left(\frac{\omega}{\tau}\right)^{2}}\right)=-\frac{\frac{2 \omega}{\tau^{2}}+4 \omega\left(\omega_{0}^{2}-\omega^{2}\right)}{\left(\omega_{0}^{2}-\omega^{2}\right)^{2}+\left(\frac{\omega}{\tau}\right)^{2}}\left(\Gamma v_{g} g_{0} \bar{\gamma}\right)^{2}=0 \\
\omega_{r}^{2}=\omega^{2}=\omega_{0}^{2}-\frac{1}{2 \tau^{2}}
\end{gathered}
$$

Assume the lifetime $1 / \tau$ is very small; substitute Equation (3.4.19) and the cavity

lifetime $\frac{1}{\tau_{\gamma}}=\Gamma v_{g} \bar{g}$ from Equation (3.3.20) into (3.4.23) to find the resonant frequency in terms of the optical loss and photon density.

$$
\begin{gathered}
\omega_{r}^{2} \square \omega_{0}^{2}=\Gamma v_{g}^{2} g_{0} \bar{\gamma} \bar{g}+\left(v_{g} g_{0} \bar{\gamma}+\frac{1}{\tau_{n}}\right)\left(\frac{1}{\tau_{\gamma}}-\Gamma v_{g} \bar{g}\right) \\
=\Gamma v_{g}^{2} g_{0} \bar{\gamma} \bar{g}+\left(v_{g} g_{0} \bar{\gamma}+\frac{1}{\tau_{n}}\right)\left(\frac{1}{\tau_{\gamma}}-\frac{1}{\tau_{\gamma}}\right) \\
=\frac{v_{g} g_{0} \bar{\gamma}}{\tau_{\gamma}} \\
\omega_{r}=\sqrt{\frac{v_{g} g_{0} \bar{\gamma}}{\tau_{\gamma}}}
\end{gathered}
$$

Using the Equation (3.4.22) to simulate and plot the frequency responses from 0 to 20 $\mathrm{GHz}$ of the DFB laser at $20^{\circ} \mathrm{C}$ at different output powers: $5 \mathrm{~mW}, 10 \mathrm{~mW}, 15 \mathrm{~mW}$ and 20mW. The resonance frequencies can be calculated using Equation (3.4.24). 


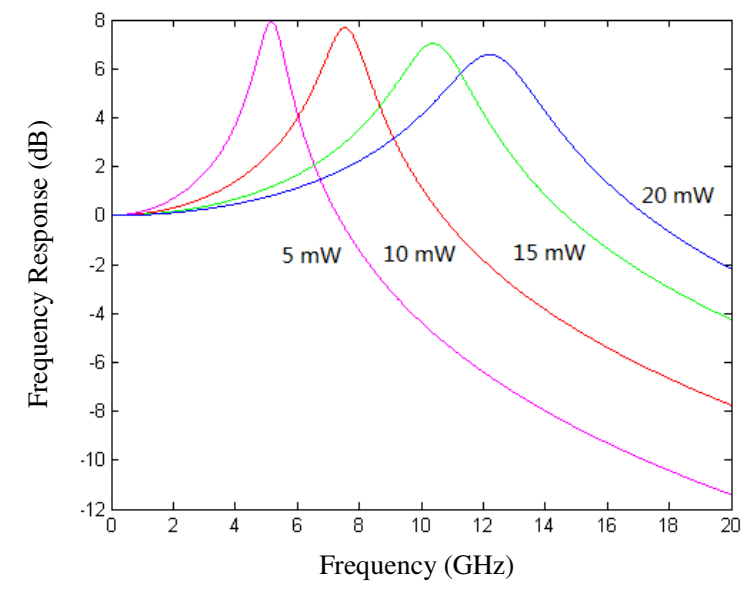

Figure 3-7: Frequency response of DFB laser at different output powers

\subsection{High Frequency Bandwidth Experimental Results}

Only the VCSELs and Fabry-Pérot lasers bandwidths have been measured.

\subsubsection{VCSELs High Frequency Bandwidths Experimental Results}

The experimental setup for to measure the VCSEL bandwidth is shown in Figure 3-8. The Network analyzer is used to measure the bandwidth of the laser by modulating the frequencies range from 0 to $20 \mathrm{GHz}$. The 5575A Bias-T from Picosecond Pulse Lab is used to mix the DC signal with an AC signal to modulate the VCSEL. The photons emitted by the VCSEL are detected by Newport D-15FC Hi-Speed Photo-detector. This signal is small in magnitude; therefore an optical amplifier has to be used to amplify the signal before being sent to port 2 of the network analyzer to obtain the frequency response. 


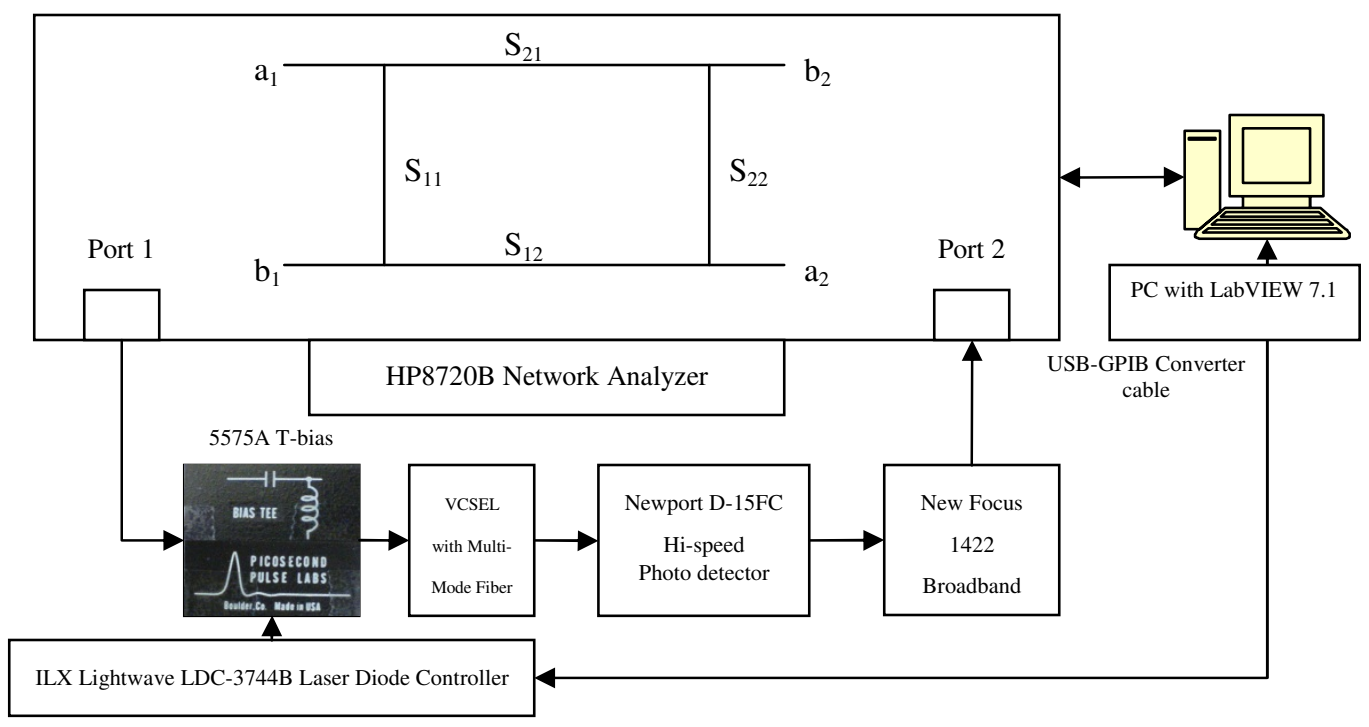

Figure 3-8: Block Diagram of the VCSEL bandwidth measurement

The frequency responses at various input currents for the 850nm VCSEL HFE4192-901 is shown in Figure 3-9. The low-power and high-power VCSEL frequency responses plots are shown in Figure 3-10 and 4-11 respectively.

Frequency Responses of VCSEL 850nm HFE4192-901

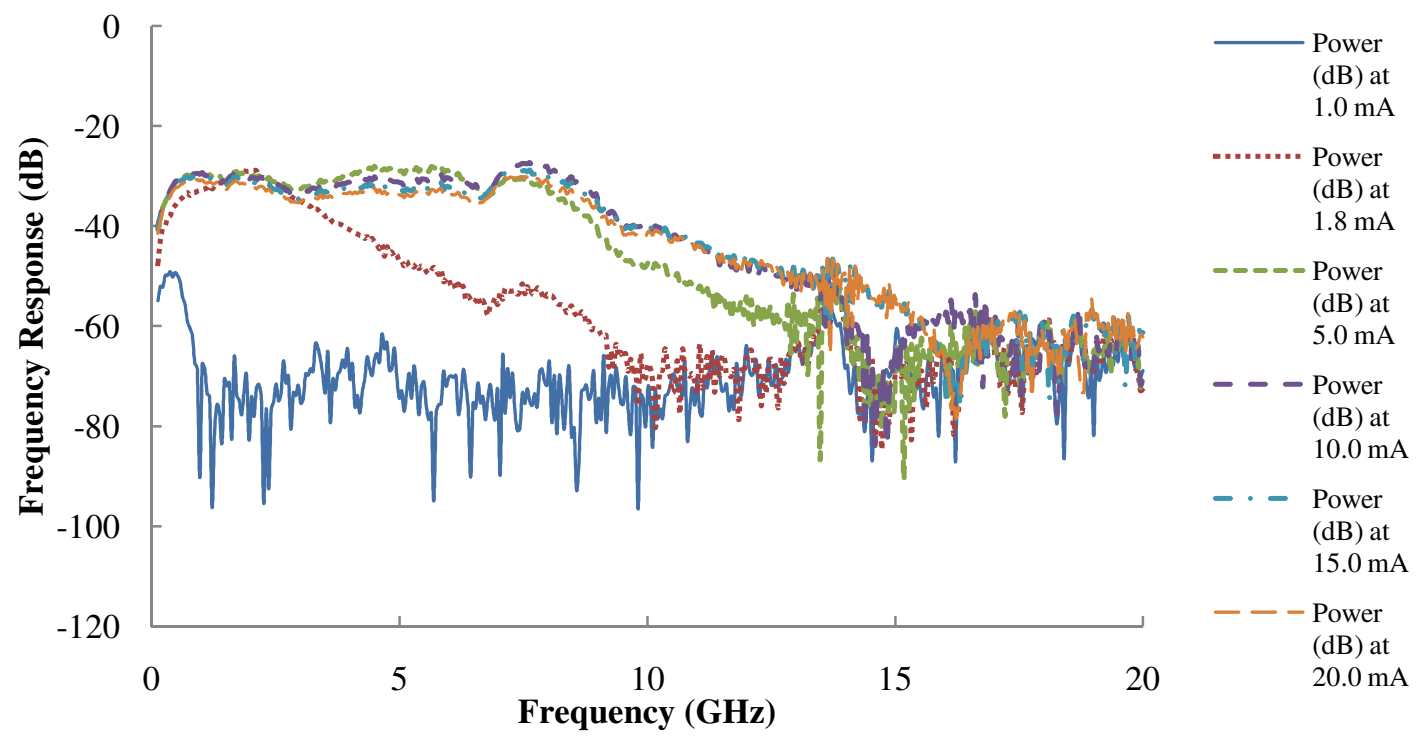

Figure 3-9: Frequency response of the default-power VCSEL 


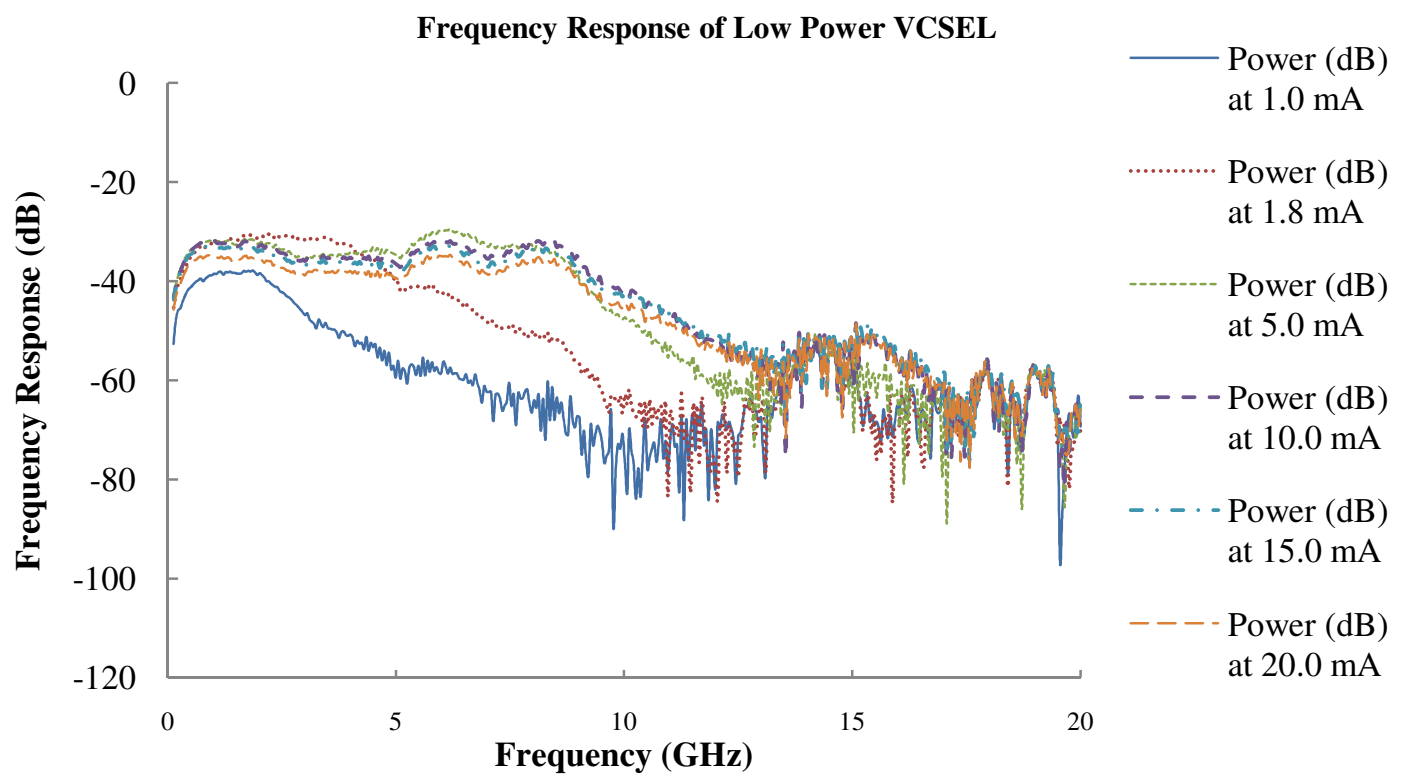

Figure 3-10: Frequency response of the low-power VCSEL

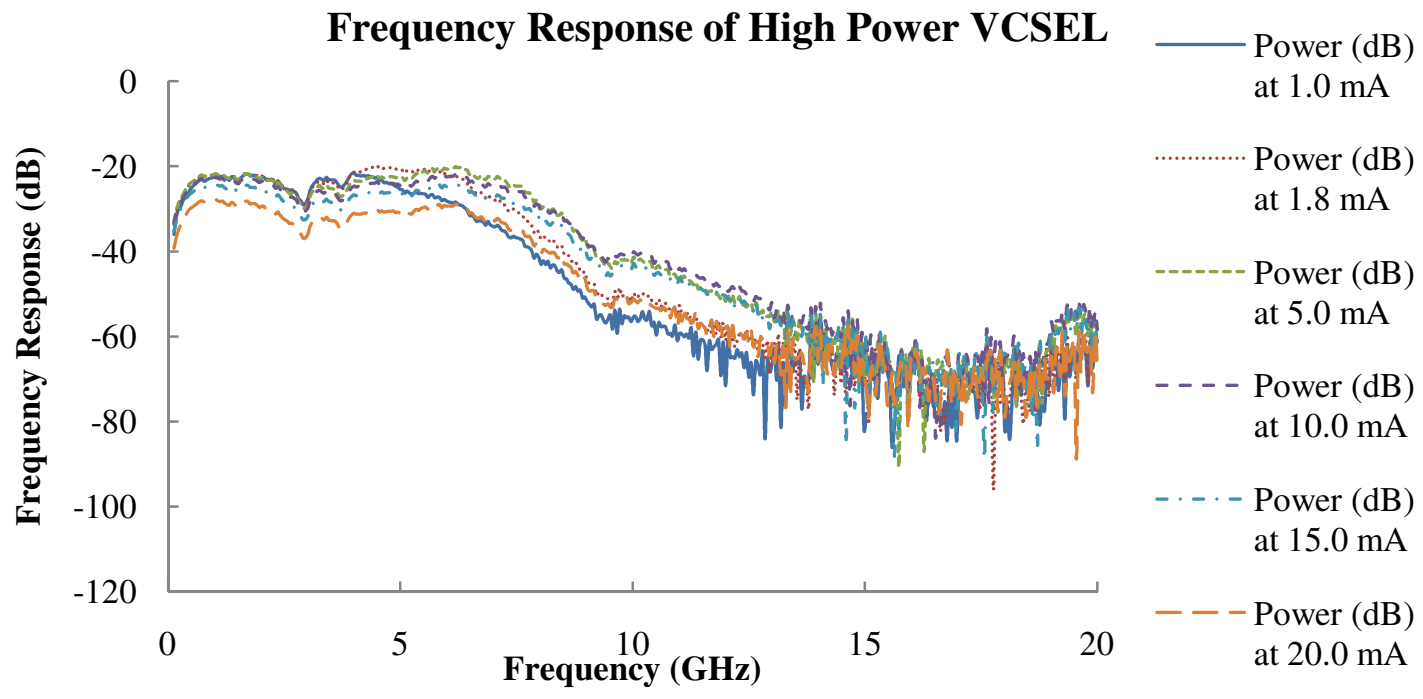

Figure 3-11: Frequency response of high-power VCSEL

The bandwidth results from Figure 3-9 to Figure 3-11 are in electrical terms. The conversion from electrical bandwidth to optical bandwidth is shown below. 


$$
f_{3 \mathrm{~dB}(\mathrm{elec})}=f_{1.5 \mathrm{~dB}(\mathrm{opt})}
$$

or

$$
f_{6 \mathrm{~dB}(\mathrm{elec})}=f_{3 \mathrm{~dB}(\mathrm{opt})}
$$

Therefore, the optical bandwidth is $6 \mathrm{~dB}$ down from the maximum gain.

The bandwidths which are $3 \mathrm{~dB}$ down from the maximum gain from the frequency responses are plotted below.

\section{Optical Bandwidths of the Default VCSEL @ 850nm}

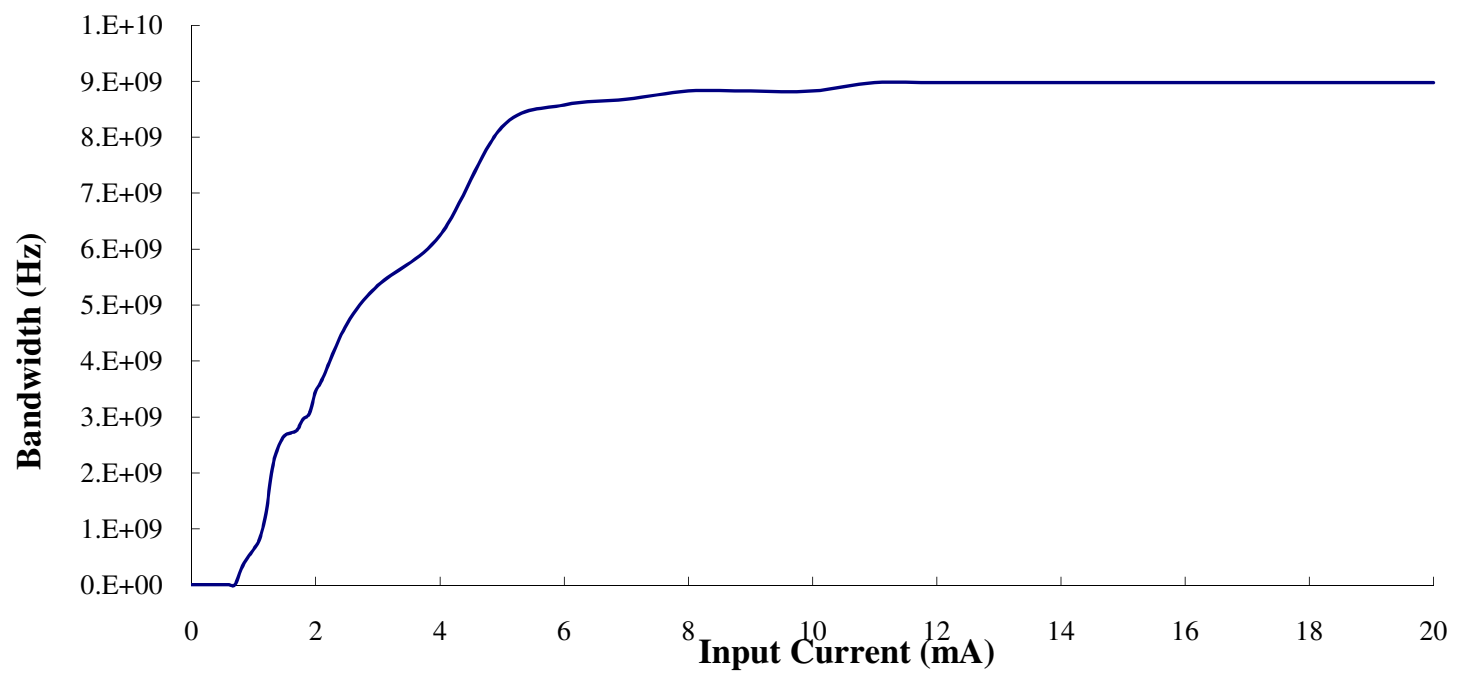

Figure 3-12: Optical bandwidths of the default-power VCSEL at 850nm 


\section{Optical Bandwidths of the Low Power VCSEL @ 850nm}

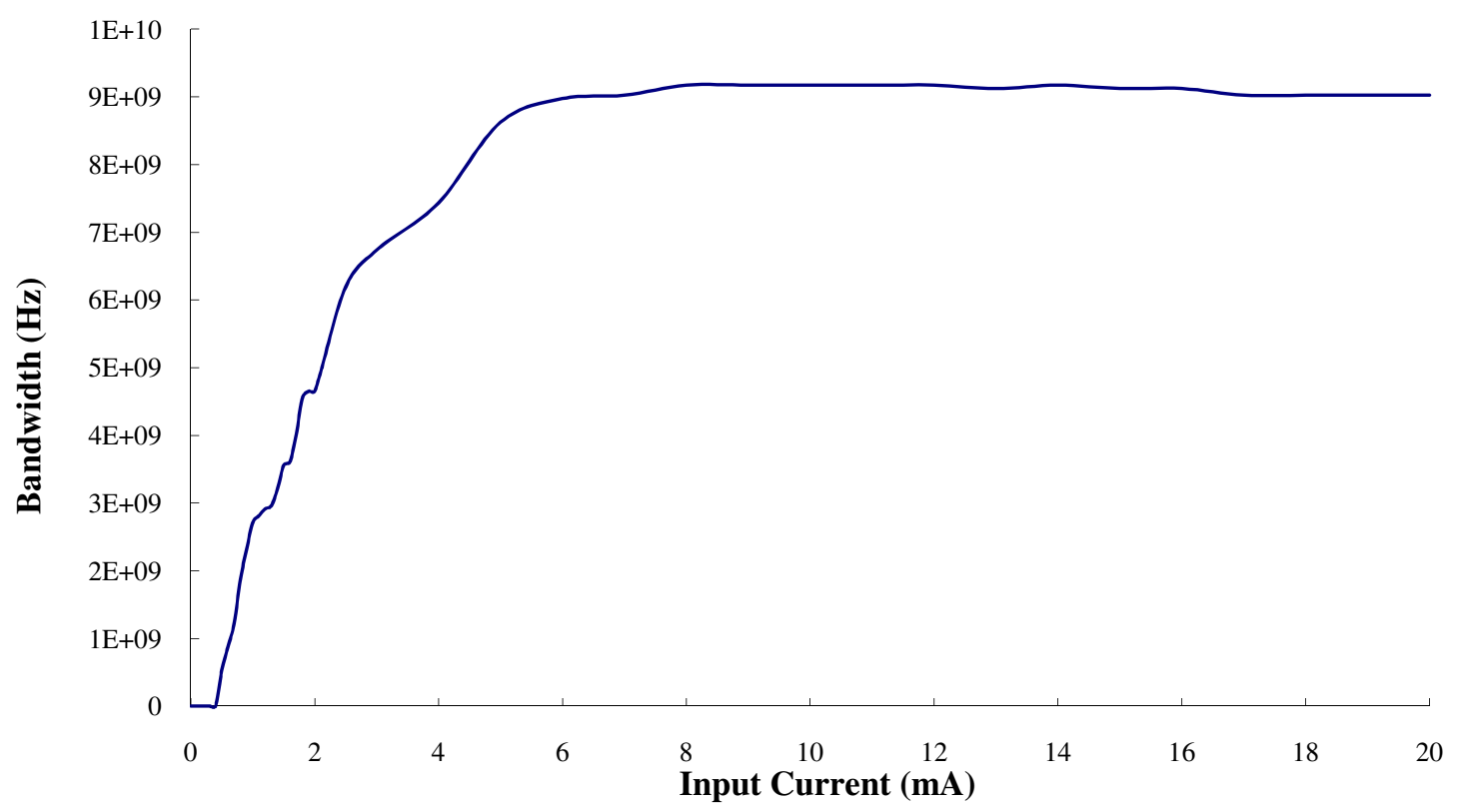

Figure 3-13: Optical bandwidths of the low-power VCSEL at 850nm

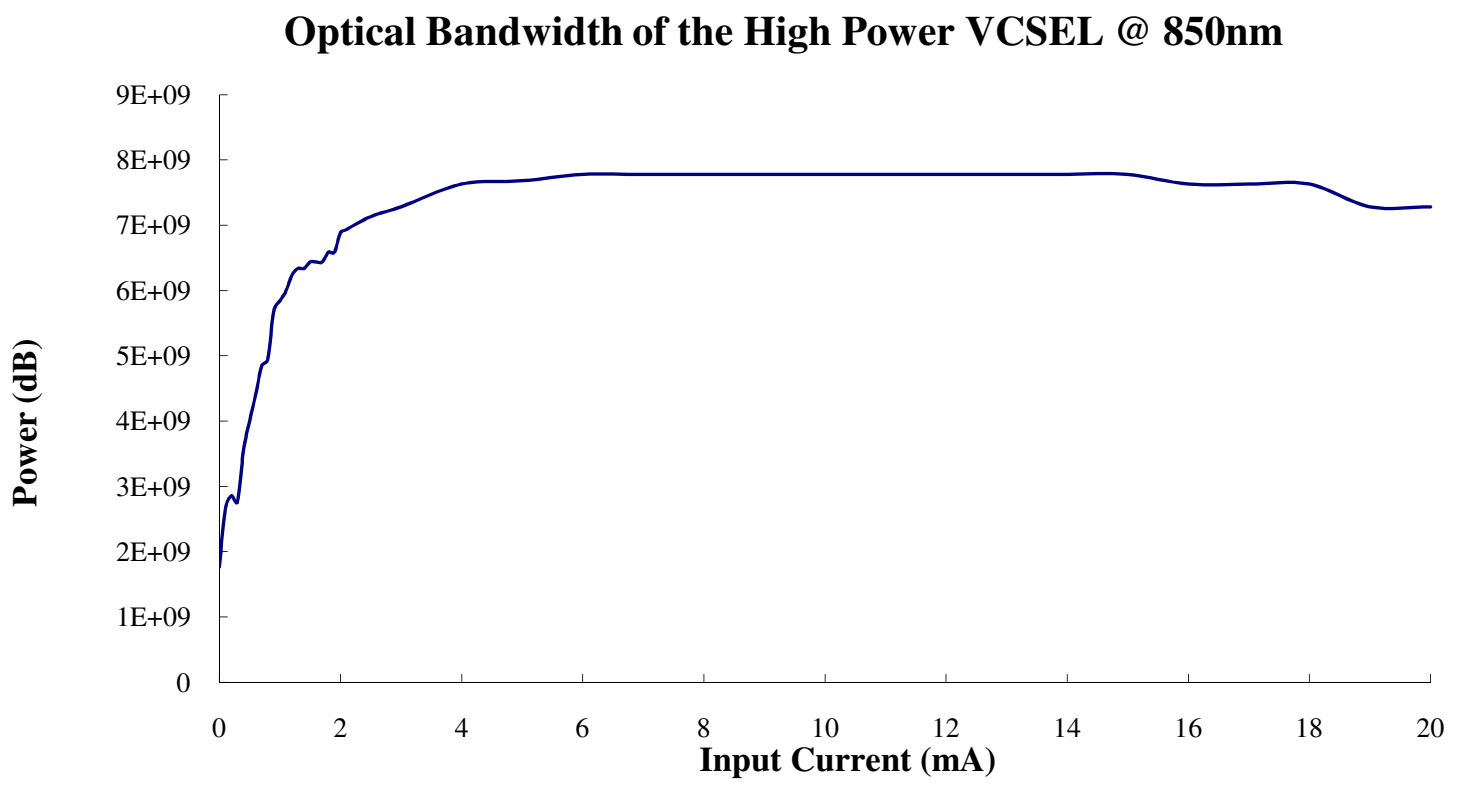

Figure 3-14: Optical bandwidths of the high-power VCSEL at 850nm 
The default power VCSEL input currents have bandwidths that are approaching to 9 GHz. The lower power VCSEL has bandwidths that go up to $9.17 \mathrm{GHz}$. Finally, the high power VCSEL has bandwidths at around $7.78 \mathrm{GHz}$.

\subsubsection{Fabry-Pérot High Frequency Bandwidths Experimental Results}

The experimental setup to measure the bandwidths of the Fabry-Pérot lasers are similar to that of the VCSELs in Figure 3-8. Instead of using the 5575A Bias-T from Picosecond Pulse Lab, it is replaced by the Fabry-Pérot laser system, where each device has its own BNC connector for signal modulation. There is no external current control on the FabryPérot laser; therefore only one bandwidth per device is recorded.

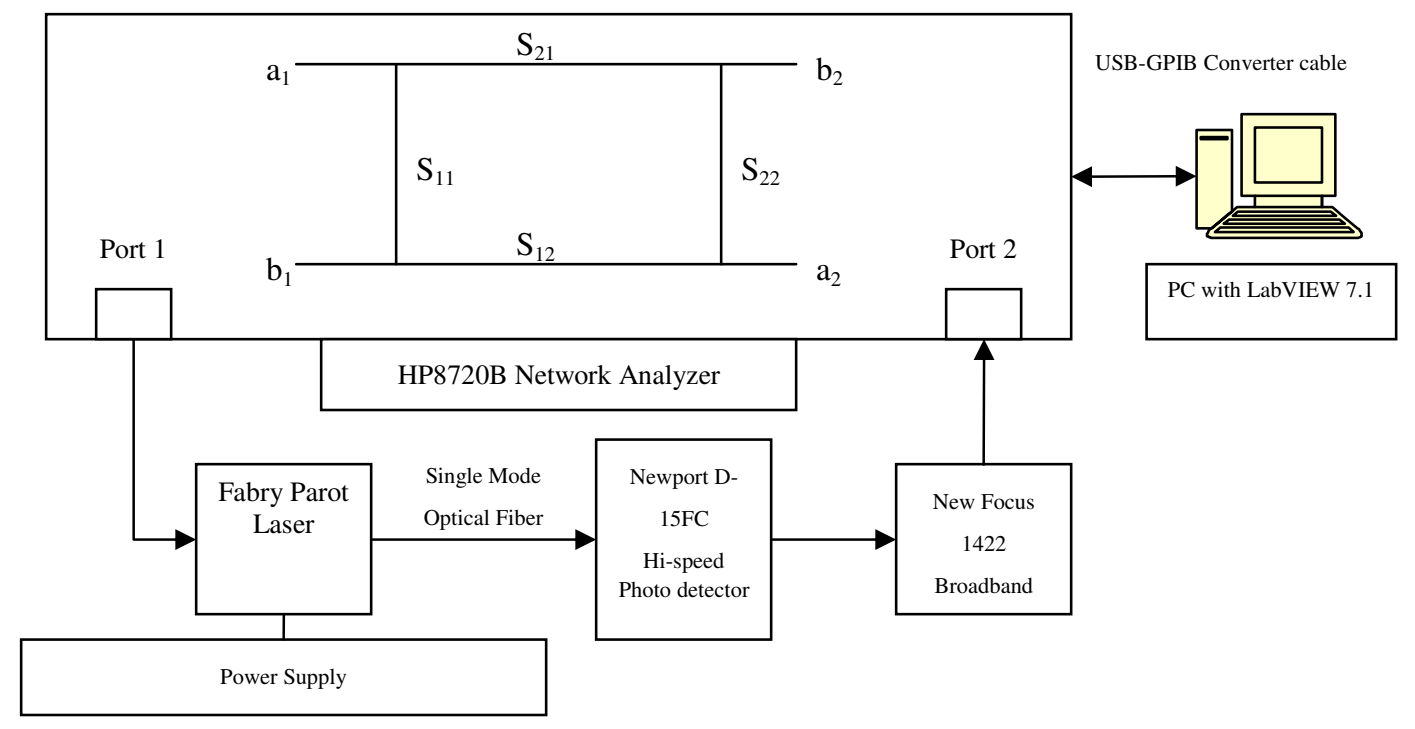

Figure 3-15: Block diagram of the Fabry-Pérot bandwidth measurement

There are four Fabry-Pérot lasers are being tested and the bandwidth plots are show below: 
\#1 Fabry-Pérot Laser Frequency Response HP83400A - S/N:

2850A00108

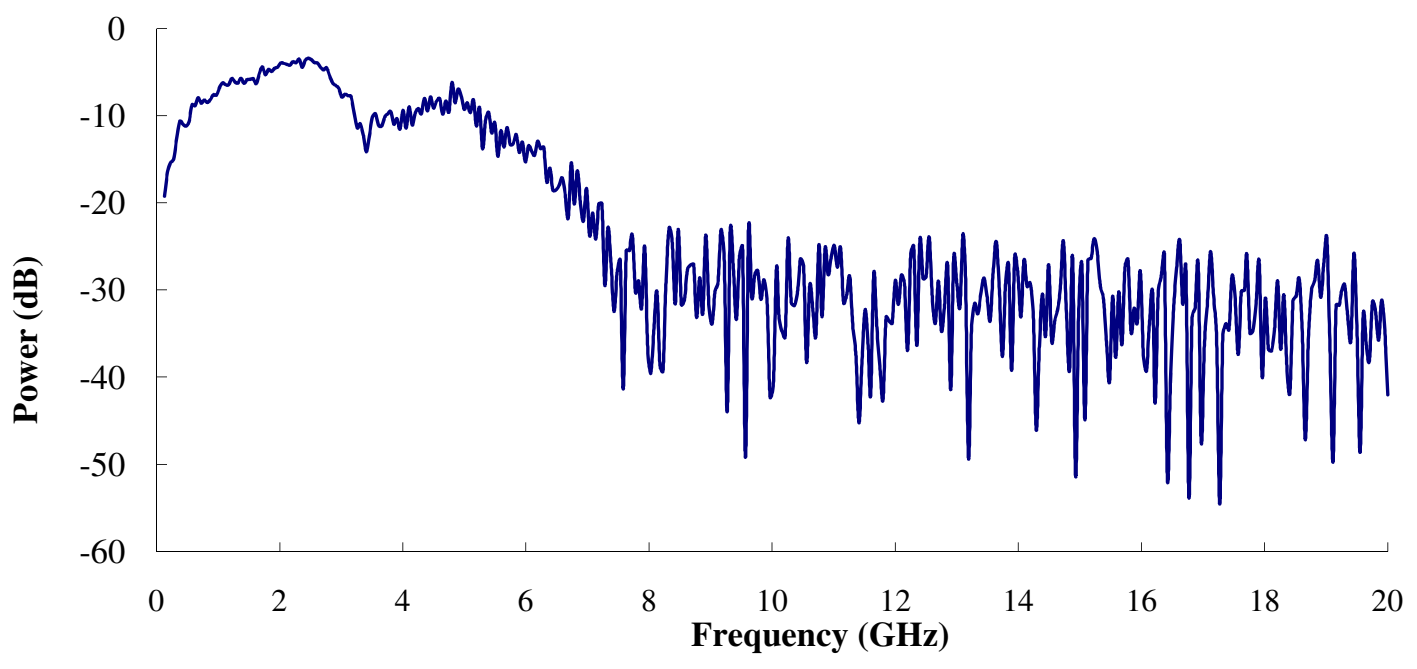

Figure 3-16: Frequency response of \#1 Fabry-Pérot laser. The bandwidth is about $3.16 \mathrm{GHz}$

\#2 Fabry-Pérot Laser Frequency Response HP83400A - Serial-2860A00114

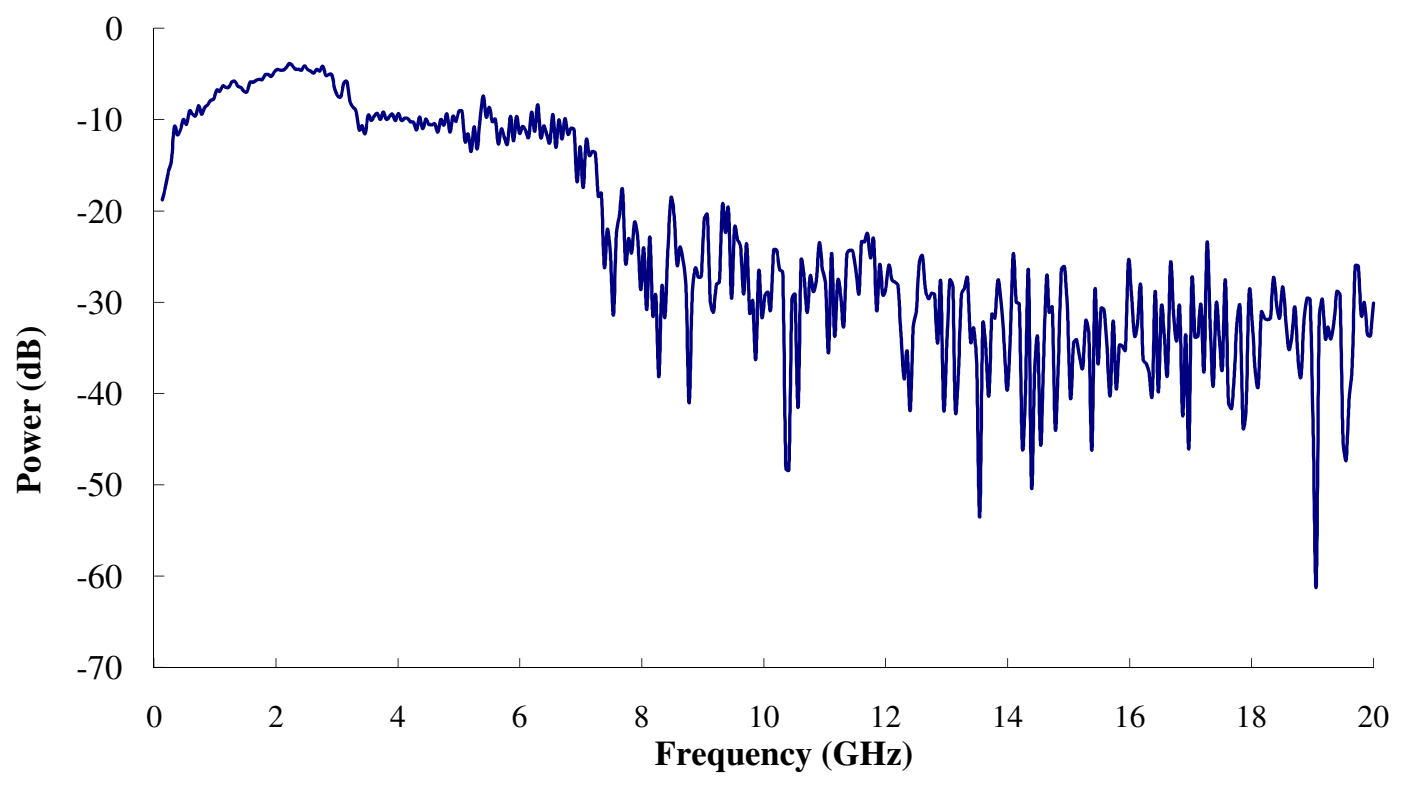

Figure 3-17: Frequency response of \#2 Fabry-Pérot laser. The bandwidth is $3.31 \mathrm{GHz}$ 


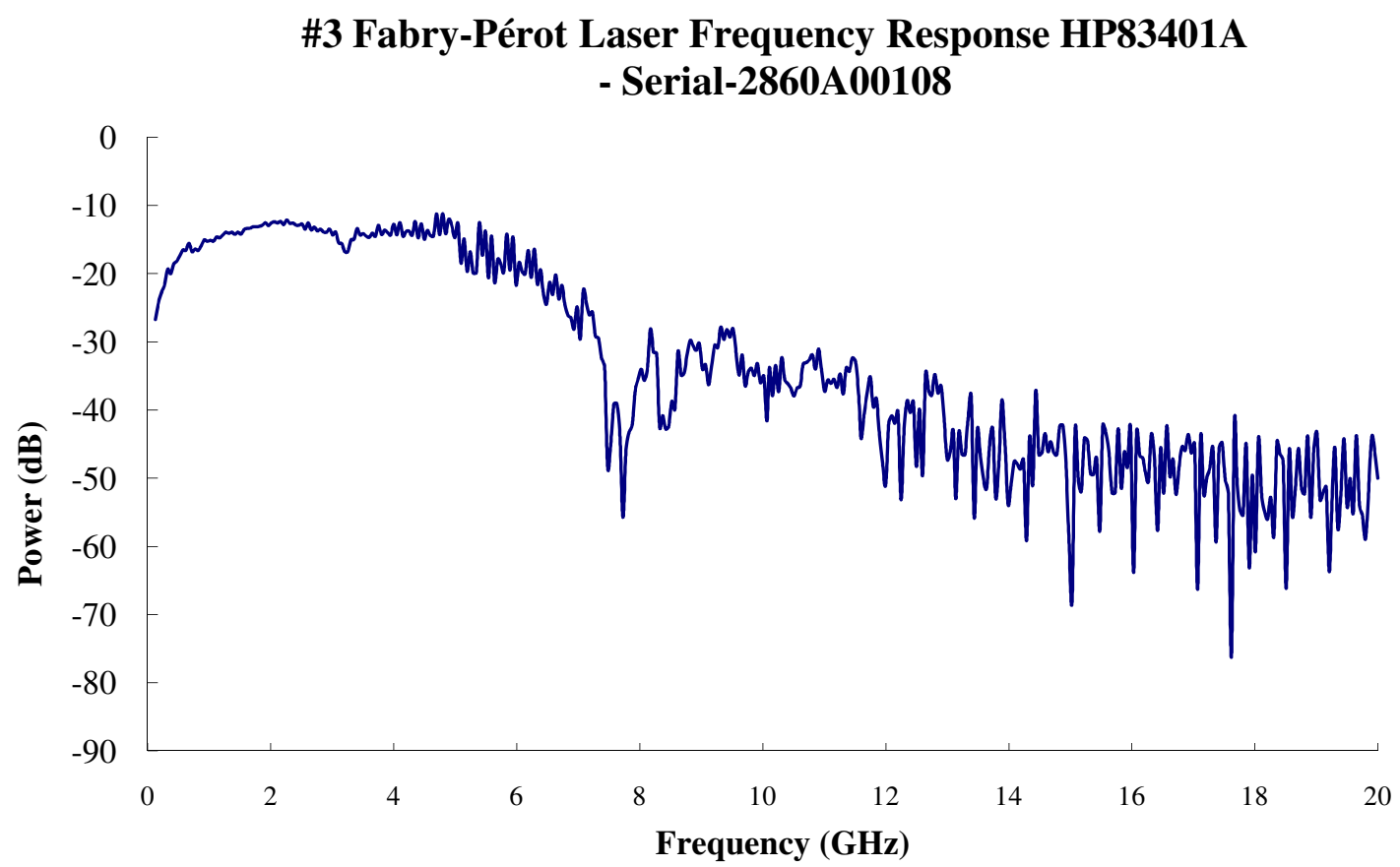

Figure 3-18: Frequency response of \#3 Fabry-Pérot laser. The bandwidth is $5.05 \mathrm{GHz}$

\#4 Fabry-Pérot Laser Frequency Response HP83402A

- Serial-2826A00203

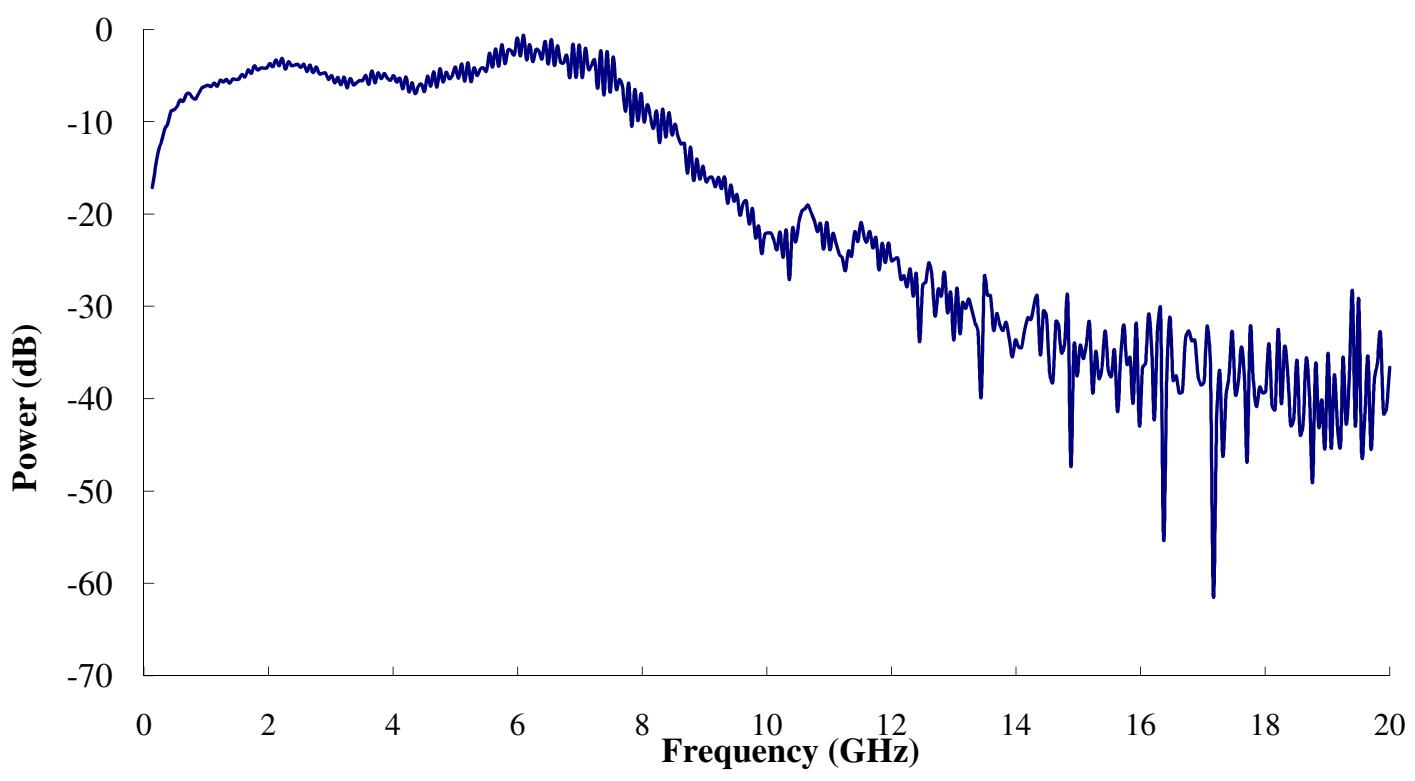

Figure 3-19: Frequency response of \#4 Fabry-Pérot laser. The bandwidth is $7.88 \mathrm{GHz}$ 
The bandwidths of the Fabry-Pérot lasers range from $3.16 \mathrm{GHz}$ to $7.88 \mathrm{GHz}$. The FabryPérot lasers frequency responses collapse when modulating at high frequencies. They are shown as large fluctuating noises in the frequency response plots. 


\section{Chapter 4: Relative Intensity Noise (RIN)}

\subsection{Introduction of Relative Intensity Noise}

The relative intensity noise describes the noise emitted by the laser. The laser noise reaches the maximum at the resonant frequency during modulation of a diode that is usually in $\mathrm{GHz}$ [2]. As the drive current increases above the threshold, the optical power output increases rapidly. Within, the laser noise changes when the drive current increases. The RIN is measure of the average noise power to the average optical power ratio expressed in $\mathrm{dB} / \mathrm{Hz}$.

\subsubsection{Experimental Setup for the RIN measurement}

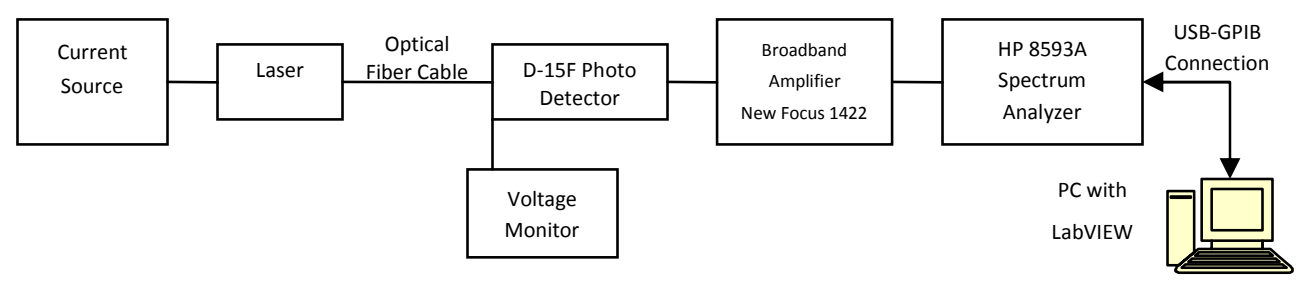

Figure 4-1: RIN spectrum measurement block diagram

The unit of the laser RIN is $\mathrm{dB} / \mathrm{Hz}, 10 \times \log \left[\frac{R I N}{\text { Unit Bandwidth }}\right]$. The HP 8593A

Spectrum Analyzer is used to measure the RIN. Not only it measures laser power output, but also the parasitic noises such as the shot noise and thermal noise that are generated in the photodetector, broadband amplifier, and inside the spectrum analyzer. Data post 
processes is needed to eliminate the parasitic noises to extract the intrinsic RIN of the laser [10].

In the experiment, only the DFB and Fabry-Pérot lasers RINs have been measured. The $\mathrm{RIN}$ is defined as:

$$
R I N=\frac{\left\langle\delta P_{0}^{2}(t)\right\rangle}{P_{0}^{2}}
$$

Where,

$\left\langle\delta P_{0}^{2}(t)\right\rangle=S_{\delta P e}(\omega) / B$ : it is the mean-squared time-averaged output optical power

(variance of the optical signal),

$P_{0}^{2}=$ the average optical power output.

The denominator of the RIN equation in (4.1.1) is related to signal-to-noise ratio of the optical power; therefore, the lower the RIN, the higher the SNR and the better the Bit Error Rate [2].

Table 4-1: Important specification of the RIN measurement setup

\begin{tabular}{|c|c|c|c|}
\hline \multicolumn{4}{|c|}{ SPECFICATIONS } \\
\hline Component & Bandwidth & $\begin{array}{c}\text { Noise } \\
\text { Figure }\end{array}$ & Gain \\
\hline $\begin{array}{l}\text { Newport D-15 } \\
\text { FC } \\
\text { Photodetector }\end{array}$ & $29 \mathrm{GHz}$ & & $\begin{array}{c}\text { Conversion } \\
\text { Gain } \mathrm{C} \geq \\
10 \mathrm{~V} / \mathrm{W}\end{array}$ \\
\hline $\begin{array}{l}\text { New Focus } \\
1422 \\
\text { Broadband } \\
\text { Amplifier }\end{array}$ & $50 \mathrm{MHz}-20 \mathrm{GHz}$ & $\mathrm{F}_{\mathrm{a}}=8 \mathrm{~dB}$ & $\begin{array}{c}\mathrm{G}_{\mathrm{a}}=18 \mathrm{~dB} \\
\text { flatness } \pm \\
2 \mathrm{~dB}\end{array}$ \\
\hline $\begin{array}{l}\text { HP8593A } \\
\text { Electrical } \\
\text { Spectrum } \\
\text { Analyzer }\end{array}$ & $\begin{array}{c}9 \mathrm{kHz}-2.9 \mathrm{GHz} \\
2.75 \mathrm{GHz}-6.5 \mathrm{GHz} \\
6 \mathrm{GHz}-12.8 \mathrm{GHz} \\
12.4 \mathrm{GHz}-19.4 \mathrm{GHz} \\
19.1 \mathrm{GHz}-22 \mathrm{GHz}\end{array}$ & $\begin{array}{l}\text { Calculated } \\
\mathrm{F}_{\mathrm{sa}}=24 \mathrm{~dB}\end{array}$ & \\
\hline
\end{tabular}


Base on the Newport D-15 photodetector specification, the conversion gain is shown in Table $4-1, \mathrm{C}=10 \mathrm{~V} / \mathrm{W}$ at $50 \Omega$ impedance match. The electrical voltage and power are defined as:

$$
\begin{gathered}
V_{e}=C P_{o p t} \\
P_{e}=\frac{V_{e}^{2}}{R} G_{a}=I_{p h}^{2} R G_{a}
\end{gathered}
$$

Where $G_{a}$ is the amplifier gain and $\mathrm{R}$ is the impedance of the load and the output in parallel resistor of the photodetector, $\mathrm{R}_{\mathrm{L}}\left\|\mathrm{R}_{\text {out }}=50 \Omega\right\| 1 \mathrm{k} \Omega=47.619 \Omega$. The current monitor conversion, $\mathrm{R}_{\mathrm{ph}}=10 \mathrm{mV} / \mu \mathrm{A}=10^{4} \Omega$, which is used to relate the photocurrent and the electric voltage measured by the digital multimeter:

$$
V_{\text {fluke }}-V_{\text {off }}=I_{p h} R_{p h}
$$

Substitute Equations (4.1.3), (4.1.4) into (4.1.2) and solve for the optical power, $P_{\text {opt }}$ :

$$
P_{o p t}=\frac{R}{C}\left(\frac{V_{\text {fluke }}-V_{o f f s e t}}{R_{p h}}\right)
$$

The electrical equivalent quantities of the RIN are expressed in the following:

$$
R I N=\frac{S_{\delta P_{e}}(\omega)}{P_{e} B}
$$

$S_{\delta P_{e}}(\omega)$ is the double-sided power spectrum density, which is defined in terms of the voltage measured by the spectrum analyzer and the impedance of the photodetector.

$$
S_{\delta P_{e}}(\omega)=\frac{V_{s a}^{2}(\omega)}{R}
$$


Set Equation (4.1.4) in terms of the photocurrent, $I_{p h}$, and substitute it into Equation

$$
P_{e}=I_{p h}^{2} R G_{a}=\left(\frac{V_{\text {fluke }}-V_{\text {offset }}}{R_{p h}}\right)^{2} R G_{a}
$$

Substitute Equation (4.1.7) and Equation (4.1.8) into Equation (4.1.6) to obtain the RIN measurement equation by the spectrum analyzer:

$$
\operatorname{RIN}_{\text {measured }}(\omega)=\left(\frac{R_{p h}}{R}\right)^{2} \frac{V_{s a}^{2}(\omega)}{G_{a} B\left(V_{\text {fluke }}-V_{\text {offset }}\right)^{2}}
$$

The variables are:

$V_{s a}(\omega)=$ voltage measured by the spectrum analyzer.

$V_{\text {fluke }}=$ voltage measured by the Fluke Digital Multimeter.

$V_{\text {offset }}=$ the offset DC voltage which is $9 \mathrm{mV}$.

$R_{p h}=10 \mathrm{mV} / \mu \mathrm{A}=10^{4} \Omega$. It is a current monitor conversion from the Newport photo

detector D-15.

$R=\mathrm{R}_{\mathrm{L}}\left\|\mathrm{R}_{\text {out }}=50 \Omega\right\| 1 \mathrm{k} \Omega$. It is the impedance of the photodetector.

$G_{a}=18 \mathrm{~dB}$. It is the amplifier gain.

$\mathrm{B}=$ the bandwidth measured by the spectrum analyzer.

$$
=\frac{R B}{2} \sqrt{\frac{\pi}{\ln 2}}=1.0645 R B=1.0645 \times 5 M H z=5.3225 M H z . R B=\text { resolution bandwidth }=
$$

$5 M H z$. FWHM (Full Width Half Max) range is $\sqrt{\pi / \ln 2}$.

The parasitic noises within the measured RIN are needed to be removed. Therefore, the shot noise and thermal noise have to be derived prior elimination. 


\subsubsection{Parasitic Shot Noise}

Shot noise is generated by the discrete nature of the emitted electrons that are causing by random fluctuations of the laser signal. When incident photons hit the cathode of the photodetector, the energy of the photons causes the emission of the photoelectrons. The photoelectrons accelerate from the cathode to the anode under the force of the electric field between the electrodes. This causes in the increase in current amplitude between the electrodes. The shot noise is modeled by a Poisson process and its variance is given by Equation (4.1.10):

$$
\overline{i_{N S}^{2}}=2 q I_{p h} B
$$

where $q$ is the magnitude of the charge on an electron, $q=1.6 \times 10^{-19} \mathrm{C}$. The power of the shot noise is defined as:

$$
P_{\text {shot }}=R \overline{i_{N S}^{2}}
$$

Substitute Equation (4.1.8) and Equation (4.1.11) into the standard RIN Equation (4.1.1),

$$
\operatorname{RIN}_{\text {shot }}=\frac{P_{\text {shot }}}{P_{e} B}
$$

Expand the RIN measurement for shot noise in terms of $\mathrm{dB} / \mathrm{Hz}$ we have,

$$
\begin{aligned}
R I N_{\text {shot }} & =\frac{R \overline{i_{N S}^{2}}}{B P_{e}}=\frac{R 2 q I_{p h} B}{B P_{e}}=\frac{R 2 q\left(\frac{V_{\text {fluke }}-V_{\text {offset }}}{R_{p h}}\right) B}{B\left(\frac{V_{\text {fluke }}-V_{\text {offset }}}{R_{p h}}\right)^{2} R G_{a}} \\
& =\frac{2 q R_{p h}}{\left(V_{\text {fluke }}-V_{\text {offset }}\right) G_{a}}
\end{aligned}
$$




\subsubsection{Parasitic Thermal Noise (Johnson or Nyquist Noise)}

Thermal noise, which is also called Nyquist noise or Johnson noise, refers to random fluctuations in the motion of charge carriers within the broadband amplifier and photodetector's resistor $R$, to cause random variations in voltage or current. The variance or the mean-square current noise that is generated by the current source and is represented by

$$
\overline{i_{t h}^{2}}=\frac{4 k T B}{R}
$$

Where $k=1.38 \times 10^{-23} \mathrm{~J} / \mathrm{K}$ is the Boltzmann constant. $T$ is the temperature in Kelvin, and $B$ is the photodetector's electrical bandwidth in $\mathrm{Hz}$.

To calculate the thermal noise, the noise figures and gains of the photodetector, amplifier and spectrum analyzer have to be used. The equation of the noise figure of a two-port network is defined as

$$
F=\frac{\text { Signal to Noise Ratio at the Input }}{\text { Signal to Noise Ratio at the Output }}=\frac{S_{i} / N_{i}}{S_{0} / N_{0}}
$$

Rewrite Equation (4.1.15) by substituting the output signal $S_{0}=G S_{i}$, where $G=$ gain, we have

$$
F=\frac{S_{i}}{S_{0}} \frac{N_{0}}{N_{i}}=\frac{S_{i}}{G S_{i}} \frac{N_{0}}{N_{i}}=\frac{N_{0}}{G N_{i}}
$$

By definition, the input noise power, $N_{i}=4 k T B$. Re-write the output noise power $N_{0}$ as

$$
N_{0}=F G N_{i}=F G(4 k T B)
$$


Equation (4.1.17) implies that the input noise power $N_{i}$ (in $\mathrm{dB}$ ) is raised by the noise figure $F($ in $\mathrm{dB}$ ) and the gain (in $\mathrm{dB}$ ) [11]. The noise figure of a component should be independent of the input noise; therefore $F$ is defined based on a standard input noise source $N_{i}$ at room temperature $T=290 \mathrm{~K}$ in a bandwidth $B$. The noise figures for the thermal noise are calculated below:

\section{Newport D-15 FC High-Speed Photodetector:}

Use noise-equivalent power (NEP) to measure the detector's sensitivity with the relationship to the amount of optical power that has a signal-to-noise ratio of unity.

$$
S N R=\frac{S_{i}}{N_{i}}=\frac{\left(\frac{\eta e}{h f} P_{\min }\right)^{2} R}{4 k T B_{n}}=1
$$

Re-arrange Equation (4.1.18) in terms of the minimum detectable power, $P_{\min }$ per square root of the system bandwidth with units in $\left[\frac{W}{H z^{1 / 2}}\right]$

$$
\begin{aligned}
N E P & =\frac{P_{\min }}{\sqrt{B_{n}}}=\frac{h f}{\eta e} \sqrt{\frac{4 k T F_{n}}{R}} \\
& =\frac{1}{\square} \sqrt{\frac{N_{n}}{R}}
\end{aligned}
$$

Where $N_{n}$ is the detector noise power, and $\square=\frac{\eta e}{h f}$ is the responsivity of the PIN photodiode with units of $[A / W]$ which is proportional to the conversion gain $C=$ $10 \mathrm{~V} / \mathrm{W}$. Use the conversion gain $C$ to solve for the responsivity: 


$$
\begin{aligned}
& \square R=C \\
& \square\left(R_{L} \| R_{\text {out }}\right)=C \\
& \square=\frac{C}{\left(R_{L} \| R_{\text {out }}\right)} \\
& \square=\frac{10 \mathrm{~V} / \mathrm{W}}{50 \Omega \| 1 \mathrm{k} \Omega}=\frac{10 \mathrm{~V} / \mathrm{W}}{47.619 \Omega}=0.21 \mathrm{~A} / \mathrm{W}
\end{aligned}
$$

Base on Equation (4.1.17), the detector noise $N_{n}=F_{n} G_{n} 4 k T B_{n}$ in equation (4.1.19), is equivalent to the thermal noise $P_{T H n}$, where $G_{n}=1$. Solve equation (4.1.20) for the noise figure $F_{n}$ first and then the thermal noise $P_{T H n}$ as shown below:

$$
\begin{aligned}
F_{n} & =N E P^{2} \square^{2} \frac{R}{4 k T} \\
& =\left(90 \frac{p W}{\sqrt{H z}} \times 0.21 \frac{A}{W}\right)^{2}\left(\frac{47.619 \Omega}{4 \times 1.38 \times 10^{-23} \frac{J}{K} \times 290 K}\right) \\
& =1.0626 \\
& =0.2637 \mathrm{~dB}
\end{aligned}
$$

Use Equation (4.1.17) to solve for the photodetector thermal noise power $P_{T H n}$.

$$
\begin{aligned}
P_{T H n} & =F_{n}\left(4 k T B_{n}\right) \\
& =(1.0626)\left(4 \times 1.38 \times 10^{-23} \frac{\mathrm{J}}{K} \times 290 \mathrm{~K} \times 29 \times 10^{9} \mathrm{~Hz}\right) \\
& =4.9329 \times 10^{-10} \mathrm{~W} \\
& =10 \log _{10}\left(\frac{4.9329 \times 10^{-10} \mathrm{~W}}{1 \mathrm{~mW}}\right) \\
& =-63.0690 \mathrm{dBm}
\end{aligned}
$$

\section{New Focus 1422 Broadband Amplifier:}

The specifications of the New Focus 1422 are provided by the product manual. 
The noise figure $F_{a}=8 \mathrm{~dB} / \mathrm{Hz}$. Bandwidth $B_{a}=1.0645 \mathrm{kHz}$. Gain, $G_{a}=18 \mathrm{~dB}$. Input noise, $N_{i}=22 \frac{p A}{\sqrt{H z}}$. Input and output impedance $R$ : $50 \Omega$. All the parameters in decibels $(\mathrm{dB})$ must be converted into linear forms before calculation. To calculate the noise power, $N_{a}$ or the thermal noise power, $P_{T H a}$,

$$
\begin{aligned}
P_{T H a} & =N_{a}=N_{i} R B_{a}=\left(22 \frac{p A}{\sqrt{H z}}\right)^{2}\left(50 \frac{J}{s A^{2}}\right)(20 G H z) \\
& =\left(22 \times 10^{-12} A^{2} \cdot s\right)\left(50 \frac{J}{s \cdot A^{2}}\right)\left(\frac{20 \times 10^{9}}{s}\right) \\
& =4.84 \times 10^{-10} \frac{J}{s} \\
& =10 \log _{10}\left(\frac{4.84 \times 10^{-10} W}{1 m W}\right) \\
& =-93.1515 \mathrm{dBW} \\
& =-63.1515 \mathrm{dBm}
\end{aligned}
$$

The amplifier noise $N_{a}$ can also be found using Equation (4.1.17). $F_{a}=10^{(8 / 10)}=$ 6.3096 and $G_{a}=10^{(18 / 10)}=63.0957$.

$$
\begin{aligned}
P_{\text {ТН }} & =N_{a}=F_{a} G_{a} N_{i}=F_{a} G_{a}\left(4 k T B_{a}\right) \\
& =(6.3096)(63.0957)\left(4 \times 1.38 \times 10^{-23} \frac{\mathrm{J}}{\mathrm{K}} \times 290 \mathrm{~K} \times 20 \times 10^{9} \mathrm{~Hz}\right) \\
& =1.2746 \times 10^{-7} \mathrm{~W} \\
& =10 \log _{10}\left(\frac{1.2746 \times 10^{-7} \mathrm{~W}}{1 \mathrm{~mW}}\right) \\
& =-68.9463 \mathrm{dBW} \\
& =-38.9463 \mathrm{dBm}
\end{aligned}
$$




\section{HP 8593A Spectrum Analyzer Noise Figure:}

Using Equation (4.1.16) and Equation (4.1.17) where $N_{0}=P_{s a}$ and $N_{i}=4 k T B$ to solve for the noise figure of the spectrum analyzer. The thermal noise power $P_{T H s a}$ of the spectrum analyzer is given as $3.98 \mathrm{fW}$ or $-114 \mathrm{dBm}$ at $2.75-6.35$ GHz with resolution bandwidth at $30 \mathrm{~Hz}$. The bandwidth $B_{s a}=1.0645 \mathrm{kHz} . \mathrm{T}=$ $290 \mathrm{~K}$.

$$
F_{s a}=\frac{F_{s a}=\frac{P_{T H s a}}{4 k T B_{s a}}}{4 \times 1.38 \times 10^{-23} \frac{\mathrm{J}}{\mathrm{K}} \times 290 \mathrm{~K} \times 1.0645 \times 10^{3} \mathrm{~Hz}}=233.5610=23.684 \mathrm{~dB}
$$

\subsubsection{Total Thermal Noise}

The total thermal noise is calculated by adding the high-speed photo detector, broadband amplifier, and the spectrum analyzer thermal noises.

$$
\begin{gathered}
P_{\text {Total Thermal }}=P_{T H n}+P_{T H a}+P_{T H s a} \\
P_{\text {Total Thermal }}=F_{n}\left(4 k T B_{n}\right)+F_{a} G_{a}\left(4 k T B_{a}\right)+P_{T H s a} \\
P_{\text {Total Thermal }}=(-63.0690 \mathrm{dBm})+(-38.9463 \mathrm{dBm})+(-114 \mathrm{dBm}) \\
=4.9329 \times 10^{-10} \mathrm{~W}+1.2746 \times 10^{-7} \mathrm{~W}+3.98 \times 10^{-15} \mathrm{~W} \\
=1.2795 \times 10^{-7} \mathrm{~W} \\
= \\
=-38.9295 \mathrm{dBm}
\end{gathered}
$$

The noise output of a cascaded system is used here. Since the thermal noise is generated by the broadband amplifier and the spectrum analyzer, the thermal noise can be calculated using a cascaded circuit with two networks as shown in Figure 4-2. 


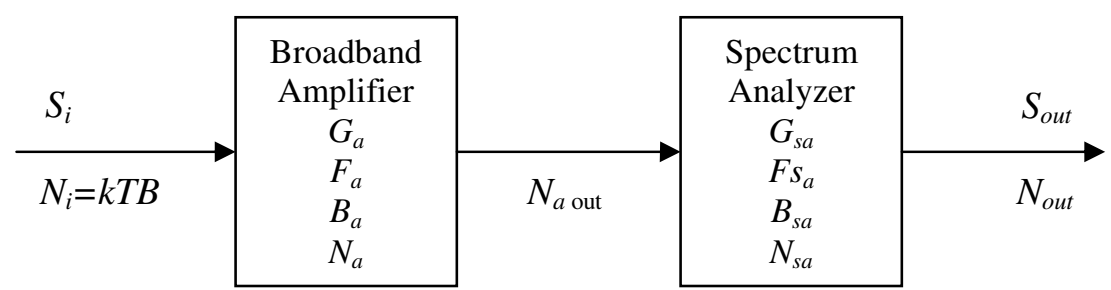

Figure 4-2: Cascaded circuit with two networks

To calculate the total output noise power, $N_{o}$, the output noise power of the New Focus 1422 Broadband Amplifier $N_{a}$ out has to be determined first by the output of the noise power:

$$
\begin{aligned}
& N_{\text {a out }}=G_{a}\left(N_{i}+N_{a}\right) \\
& N_{\text {out }}=G_{\text {sa }}\left(N_{a \text { out }}+N_{\text {sa }}\right)
\end{aligned}
$$

The noise figure of a single network is

$$
\begin{aligned}
& F=\frac{S_{i} / N_{i}}{S_{o} / N_{o}}=\frac{S_{i}}{N_{i}} \frac{N_{o}}{S_{o}}=\frac{S_{i}}{N_{i}} \frac{G_{a}\left(N_{i}+N_{o}\right)}{G_{a} S_{i}} \\
& F=1+\frac{N_{o}}{N_{i}}
\end{aligned}
$$

For a cascaded network, use Equation (4.1.30) to solve for the noise figures for the broadband amplifier and the spectrum analyzer blocks:

$$
\begin{aligned}
& F_{a}=1+\frac{N_{a}}{N_{i}} \\
& N_{a}=\left(F_{a}-1\right) N_{i} \\
& F_{s a}=1+\frac{N_{s a}}{N_{i}} \\
& N_{s a}=\left(F_{s a}-1\right) N_{i}
\end{aligned}
$$


Substitute Equations (4.1.32) into (4.1.28):

$$
N_{a \text { out }}=G_{a} N_{i}\left[1+\left(F_{a}-1\right)\right]
$$

Substitute Equations (4.1.34) and (4.1.35) into (4.1.29):

$$
N_{\text {out }}=G_{a} G_{s a} N_{i}\left[F_{a}+\frac{\left(F_{s a}-1\right)}{G_{a}}\right]
$$

Since there is no gain in the spectrum analyzer, so $G_{s a}=1$ and Equation (4.1.36) becomes

$$
P_{\text {total thermal }}=N_{\text {out }}=4 k T B G_{a}\left[F_{a}+\frac{\left(F_{s a}-1\right)}{G_{a}}\right]
$$

The RIN of thermal noise is

$$
\begin{aligned}
\operatorname{RIN}_{\text {Total Thermal }}= & \frac{P_{\text {Total Thermal }}}{P_{e} B} \\
& =\frac{4 k T B G_{a}\left[F_{a}+\frac{\left(F_{s a}-1\right)}{G_{a}}\right]}{\left(\frac{V_{\text {fluke }}-V_{\text {offset }}}{R_{p h}}\right)^{2} R G_{a} B} \\
& =\frac{4 k T\left[F_{a}+\frac{\left(F_{s a}-1\right)}{G_{a}}\right]}{\left(\frac{V_{\text {fluke }}-V_{\text {offset }}}{R_{p h}}\right)^{2} R} \quad\left[\frac{W}{H z}\right]
\end{aligned}
$$




\subsubsection{Intrinsic RIN of the DFB Laser}

The intrinsic RIN of the laser is extracted by subtracting the parasitic RINs such as the RIN of the shot and thermal noises from the measured RIN. First rewriting Equation (4.1.9) using Equation (4.1.7), $S_{\delta P_{e}}(\omega)=V_{s a}^{2}(\omega) / R$ and then substituting Equation (4.1.9), (4.1.13) and (4.1.38) into the equation below:

$\operatorname{RIN}_{\text {laser }}(\omega)=($ RIN of Measured System $)-($ RIN of Shot Noise $)-($ RIN of Thermal Noise $)$

From Equation (4.1.38):

$$
\begin{gathered}
\operatorname{RIN}_{\text {laser }}(\omega)=\left(\frac{R_{p h}}{V_{\text {fluke }}-V_{\text {offset }}}\right)^{2} \frac{S_{\delta P_{e}}(\omega)}{G_{a} B R}-\frac{2 q R_{p h}}{\left(V_{\text {fluke }}-V_{\text {offset }}\right) G_{a}}-\frac{P_{\text {Total Thermal }}}{\left(\frac{V_{\text {fluke }}-V_{\text {offset }}}{R_{\text {ph }}}\right)^{2} R G_{a} B} \\
=\left(\frac{R_{p h}}{V_{\text {fluke }}-V_{\text {offset }}}\right)^{2} \frac{S_{\delta P_{e}}(\omega)}{G_{a} B R}-\frac{2 q R_{p h}}{\left(V_{\text {fluke }}-V_{\text {offset }}\right) G_{a}}-\frac{4 k T\left[F_{a}+\frac{\left(F_{\text {sa }}-1\right)}{G_{a}}\right]}{\left(\frac{\left.V_{\text {fluke }}-V_{\text {offset }}\right)^{2}}{R_{\text {ph }}} R\right.} \\
\operatorname{RIN}_{\text {laser }}[d B / H z]=10 \log _{10}\left[\operatorname{RIN}_{\text {laser }}(\omega)\right]
\end{gathered}
$$

\subsection{DFB Laser RIN Experimental Setup}

The DFB laser RIN measurement block diagram is shown in Figure 4-3. The Kette current source provides the injection current for the DFB laser. The DFB laser is then connected to a high speed photodetector Newport D-15FC via a single-mode FC optical fiber cable and the optical power is converted into a photocurrent. The optical power signal is amplified using a broadband amplifier, New Focus 1422. The amplified signal 
is sent to the HP 8593A Spectrum Analyzer. The measured power spectrum densities $S_{\delta P_{e}}(\omega)$, are then recorded into spreadsheets by the PC running LabVIEW that is interfaced with the USB-GPIB connection. The LabVIEW program records 401 power spectrum density data points from $2.75 \mathrm{GHz}$ to $22 \mathrm{GHz}$. Equation (4.1.39) to and (4.1.40) are used to create the plots of the DFB laser RIN. The power spectrum density $S_{\delta P_{e}}(\omega)$ values are needed to be calibrated to shift the entire frequency response downward starting from $6.35 \mathrm{GHz}$ to $22 \mathrm{GHz}$ with the drive currents from $5 \mathrm{~mA}$ to $48 \mathrm{~mA}$. The calibration is done by subtracting all the frequency responses at or above $6.35 \mathrm{GHz}$ down to the difference between the responses at $6.30 \mathrm{GHz}$ and $6.35 \mathrm{GHz}$.

Table 4-2: Calibrated Freq. Responses at 6.35 GHz for the DFB laser RIN plots

\begin{tabular}{|c|c|c|c|c|c|c|c|c|c|c|}
\hline \multirow[b]{2}{*}{$\begin{array}{c}\text { Frequency } \\
\text { [GHz] }\end{array}$} & \multicolumn{10}{|c|}{ Calibrated Frequency Responses at $6.35 \mathrm{GHz}$ at Different Drive Currents } \\
\hline & $\begin{array}{c}\text { Power } \\
\text { [dBm] } \\
@ \\
5 \mathrm{~mA}\end{array}$ & $\begin{array}{c}\text { Power } \\
{[\mathrm{dBm}]} \\
@ \\
10 \mathrm{~mA}\end{array}$ & $\begin{array}{c}\text { Power } \\
\text { [dBm] } \\
@ @ \\
15 \mathrm{~mA}\end{array}$ & $\begin{array}{c}\text { Power } \\
\text { [dBm] } \\
@ \\
20 \mathrm{~mA}\end{array}$ & $\begin{array}{c}\text { Power } \\
\text { [dBm] } \\
@ @ \\
25 \mathrm{~mA}\end{array}$ & $\begin{array}{c}\text { Power } \\
\text { [dBm] } \\
@ \\
30 \mathrm{~mA}\end{array}$ & $\begin{array}{c}\text { [dBm] } \\
@ \\
35 \mathrm{~mA}\end{array}$ & $\begin{array}{c}{[\mathrm{dBm}]} \\
@ \\
40 \mathrm{~mA}\end{array}$ & $\begin{array}{c}\text { Power } \\
\text { [dBm] } \\
@ \\
45 \mathrm{~mA}\end{array}$ & $\begin{array}{c}\text { Power } \\
\text { [dBm] } \\
@ \\
\mathbf{4 8 m A}\end{array}$ \\
\hline 6.35 & 7.47 & 7.76 & 6.89 & 7.56 & 7.49 & 6.12 & 4.15 & 4.22 & 5.06 & 6.96 \\
\hline
\end{tabular}

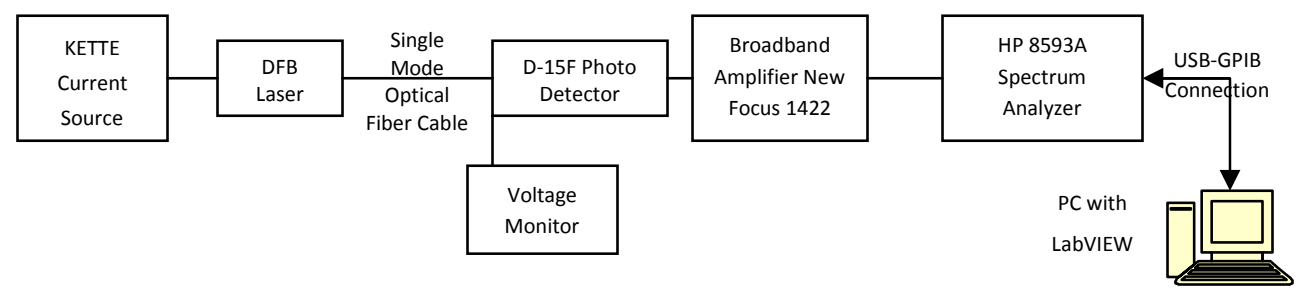

Figure 4-3: Block diagram of DFB laser relative intensity noise (RIN) 


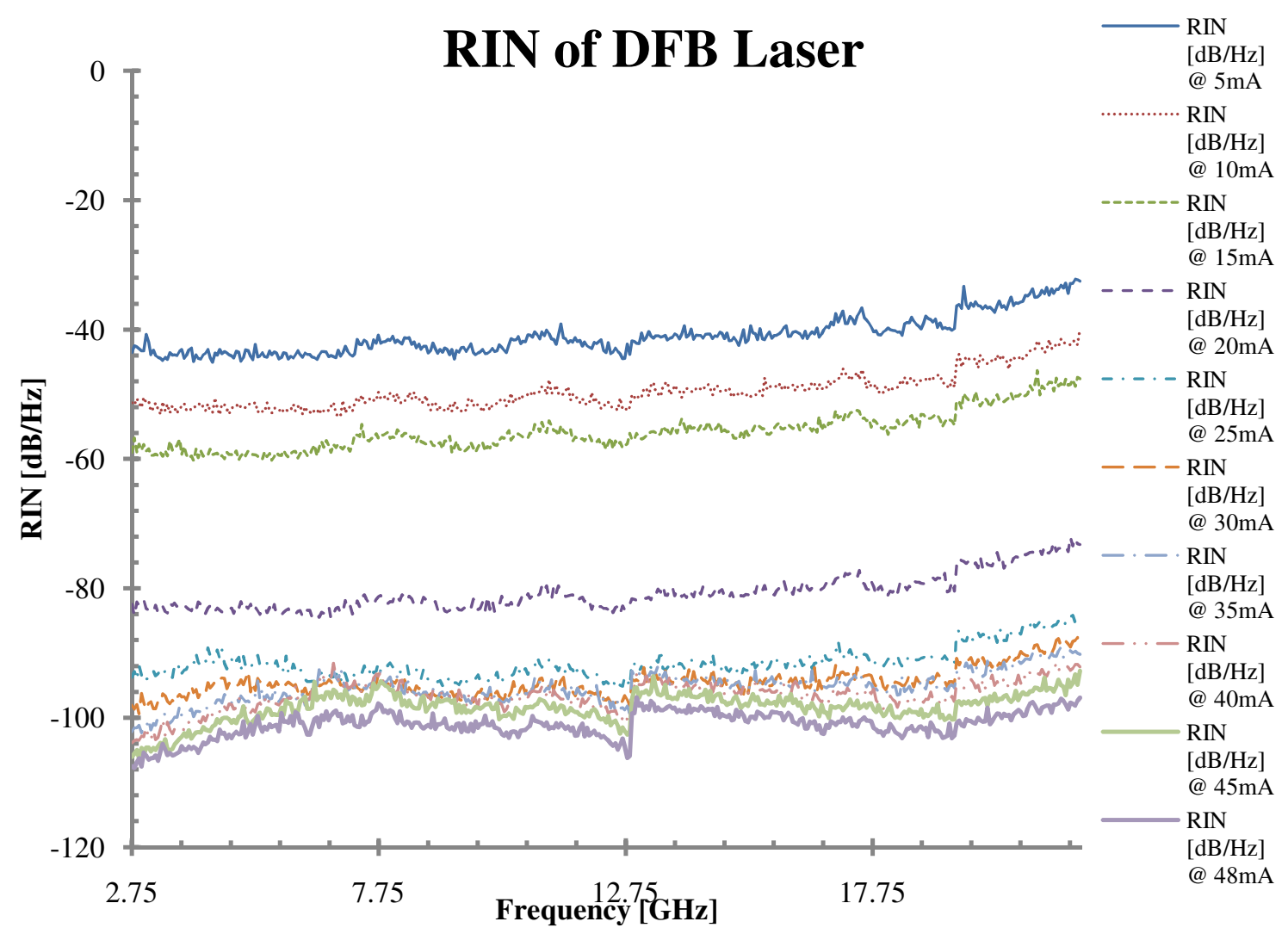

Figure 4-4: RIN plots of the DFB laser at various input currents from $5 \mathrm{~mA}$ to $48 \mathrm{~mA}$

The RIN of the DFB laser plot shows that as the drive current increasing from $5 \mathrm{~mA}$ to $48 \mathrm{~mA}$, the $\mathrm{RIN}$ in $\mathrm{dB} / \mathrm{Hz}$ decreases. The drive current helps to improve the SNR of the DFB laser. 


\subsection{Fabry-Pérot Laser RIN Experimental Setup}

The experimental setup is similar to the DFB laser with an additional amplifier

Picosecond Pulse Labs 12.5 Gbps Model 5865 to amplify the signal in order to obtain the noise. The block diagram should be is a three cascaded system.

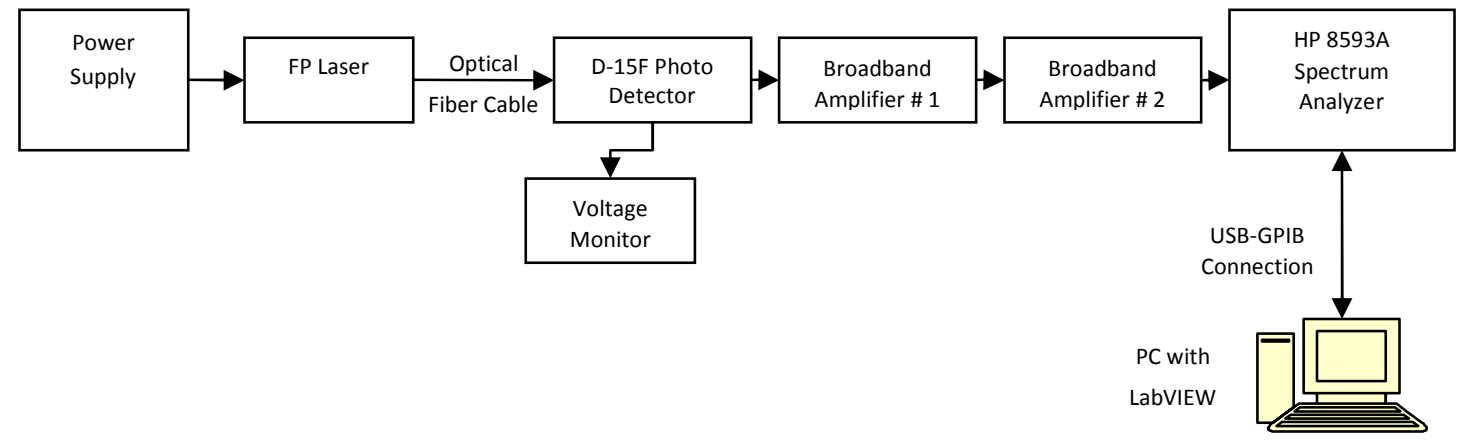

Figure 4-5: Block diagram of the Fabry-Pérot laser experimental setup

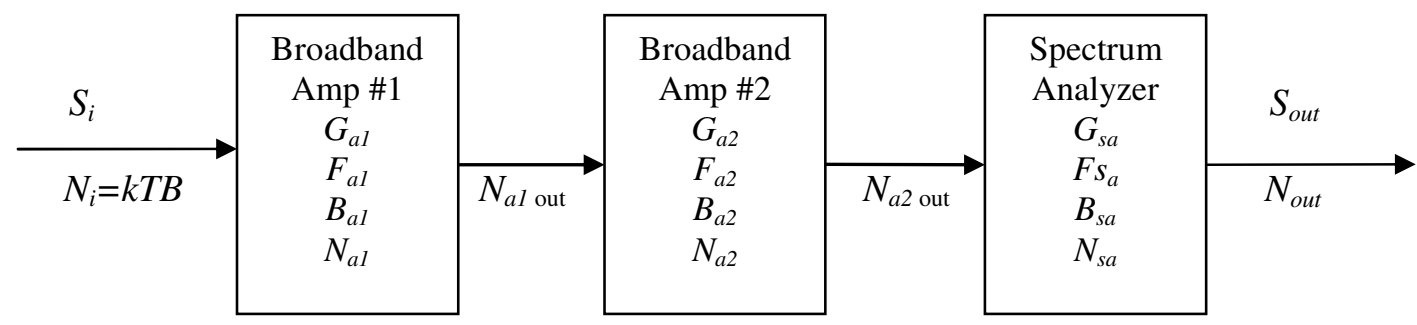

Figure 4-6: Block diagram of a cascaded circuit with three networks

The parameters of the amplifiers that are being used are shown below:

Table 4-3: Amplifiers Parameters for Fabry-Pérot laser RIN measurement

\begin{tabular}{|l|l|c|c|c|c|c|}
\hline Index & Brand & Feature & Model & $\begin{array}{c}\mathbf{S}_{\mathbf{2 1}} \text { Gain, } \\
\mathbf{G}_{\mathbf{a}}[\mathbf{d B}]\end{array}$ & $\begin{array}{c}\text { Noise } \\
\text { Figure, } \\
{[\mathbf{d B}]}\end{array}$ & $\begin{array}{c}\text { Bandwidth, } \\
\mathbf{B}_{\mathbf{a}}[\mathbf{G H z}]\end{array}$ \\
\hline$\# 1, \mathrm{a} 1$ & New Focus & $\begin{array}{c}\text { Broadband } \\
\text { Amplifier }\end{array}$ & 1422 & 18 & 8 & 20 \\
\hline \#2, a2 & $\begin{array}{c}\text { Picosecond } \\
\text { Pulse Labs }\end{array}$ & $\begin{array}{c}12.5 \mathrm{Gbps} \\
\text { Driver } \\
\text { Amplifier }\end{array}$ & Model 5865 & 26.5 & 5.75 & 12 \\
\hline
\end{tabular}


Modifying Equation (4.1.13) for shot noise to include the gain $G_{a 2}$ for amplifier \#2 -

Picosecond Pulse Labs 12.5 Gbps Driver Amplifier:

$$
R_{\text {shot }}=\frac{2 q R_{p h}}{G_{a 1} G_{a 2}\left(V_{\text {fluke }}-V_{\text {offset }}\right)}
$$

Modify Equation (4.1.36) to support three networks cascaded system, the thermal noise power at the output $N_{\text {out }}$ is,

$$
P_{\text {th }}=N_{\text {out }}=G_{a 1} G_{a 2} G_{s a} N_{i}\left[F_{a}+\frac{\left(F_{a 2}-1\right)}{G_{a 1}}+\frac{\left(F_{s a}-1\right)}{G_{a 1} G_{a 2}}\right]
$$

The RIN due to the thermal noise is

$$
\begin{aligned}
\operatorname{RIN}_{\text {thermal }}= & \frac{P_{t h}}{P_{e} B} \\
= & \frac{P_{t h}}{I_{p h}^{2} R G_{a 1} G_{a 2} G_{s a} B} \\
= & \frac{G_{a 1} G_{a 2} G_{s a} N_{i}\left[F_{a 1}+\frac{\left(F_{a 2}-1\right)}{G_{a 1}}+\frac{\left(F_{s a}-1\right)}{G_{a 1} G_{a 2}}\right]}{\left(\frac{V_{\text {fluke }}-V_{\text {offset }}}{R_{p h}}\right)^{2} R G_{a 1} G_{a 2} G_{s a} B} \\
= & \frac{4 k T\left[F_{a 1}+\frac{\left(F_{a 2}-1\right)}{G_{a 1}}+\frac{\left(F_{s a}-1\right)}{G_{a 1} G_{a 2}}\right] R_{p h}^{2}}{\left(V_{\text {fluke }}-V_{\text {offset }}\right)^{2} R}
\end{aligned}
$$

Where the bandwidth, $B=\frac{R B}{2} \sqrt{\frac{\pi}{\ln 2}}=R B 1.0645=3 \times 1.0645 \mathrm{MHz}=3.19 \mathrm{MHz}$.

The intrinsic RIN of the FP laser is extracted by subtracting the RIN of the shot and thermal noises, $R I N_{\text {shot }}$ and RIN $_{\text {thermal }}$ respectively. 


$$
\begin{aligned}
& R_{\text {laser }}= R I N_{\text {measured }}-R I N_{\text {shot }}-R I N_{\text {thermal }} \\
&=\left(\frac{R_{p h}}{V_{\text {fluke }}-V_{\text {offset }}}\right)^{2} \frac{S_{\delta P_{e}}(\omega)}{G_{a 1} G_{a 2} R B}-\frac{2 q R_{\text {ph }}}{G_{a 1} G_{a 2}\left(V_{\text {fluke }}-V_{\text {offset }}\right)}-\frac{P_{\text {Total Thermal }}}{\left(\frac{V_{\text {fluke }}-V_{\text {offset }}}{R_{p h}}\right)^{2} R G_{a 1} G_{a 2} B} \\
&=\left(\frac{R_{p h}}{V_{\text {fluke }}-V_{\text {offset }}}\right)^{2} \frac{S_{\delta P_{e}}(\omega)}{G_{a 1} G_{a 2} R B}-\frac{2 q R_{p h}}{G_{a 1} G_{a 2}\left(V_{\text {fluke }}-V_{\text {offset }}\right)}-\frac{4 k T\left[F_{a}+\frac{\left(F_{\text {sa }}-1\right)}{G_{a}}\right]}{\left(\frac{\left.V_{\text {fluke }}-V_{\text {offet }}\right)^{2}}{R_{p h}} R\right.} \\
& R I N_{\text {laser }}[d B / H z]=10 \log _{10}\left[R I N_{\text {laser }}(\omega)\right]
\end{aligned}
$$

The $R I N_{\text {laser }}$ becomes negative where $R I N_{\text {measured }}<\left(R I N_{\text {shot }}+R I N_{\text {thermal }}\right)$ which is physically unrealistic when the gain of the Picosecond Pulse Labs 5865 12Gbps Drive Amplifier $G_{a 2}$ is $26.5 \mathrm{~dB}$. 
The maximum gain value that provides the positive RIN for the laser has to be found. By tweaking the $G_{a 2}$ to $15.5 \mathrm{~dB}$ or lower, the RIN of the laser becomes positive. The minimum estimates of the Fabry-Pérot laser RINs are plotted below:

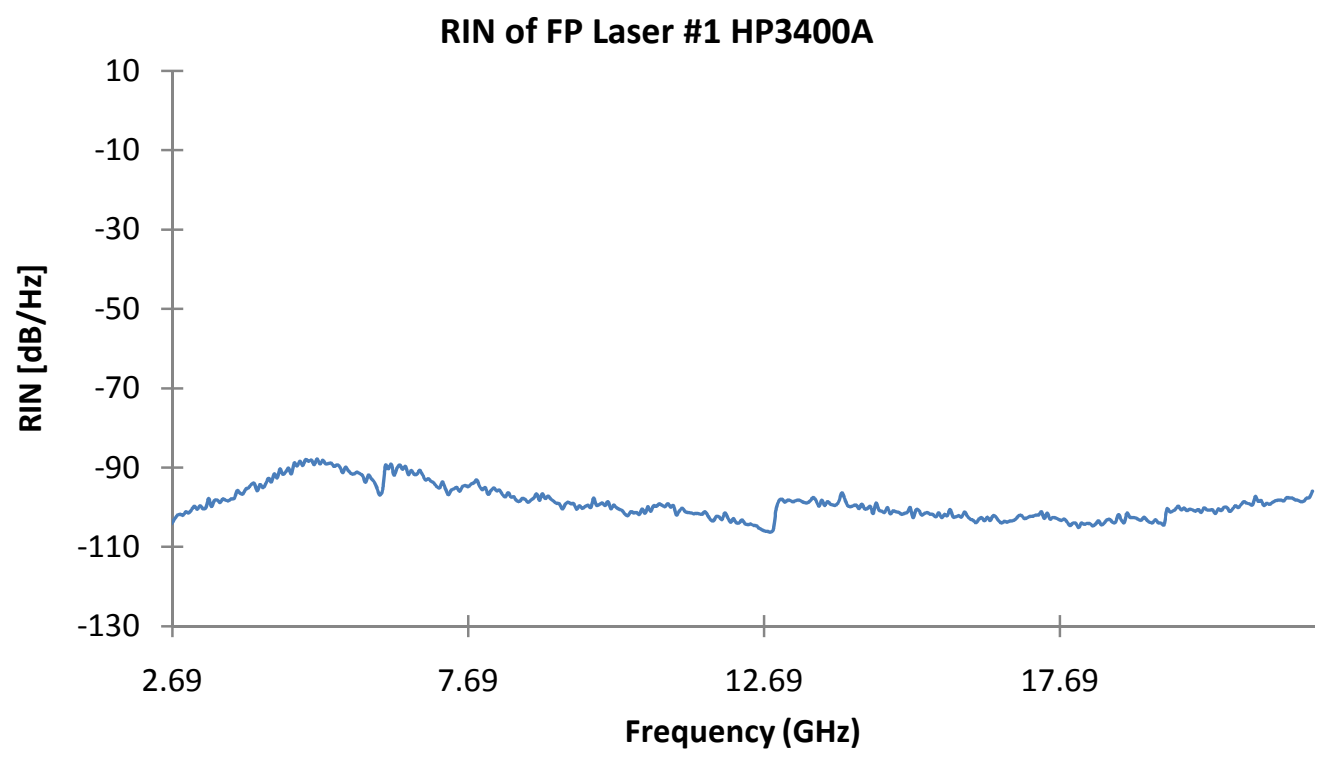

Figure 4-7: RIN of FP laser \#1 HP3400A at $G_{a 2}=15.5 \mathrm{~dB}$

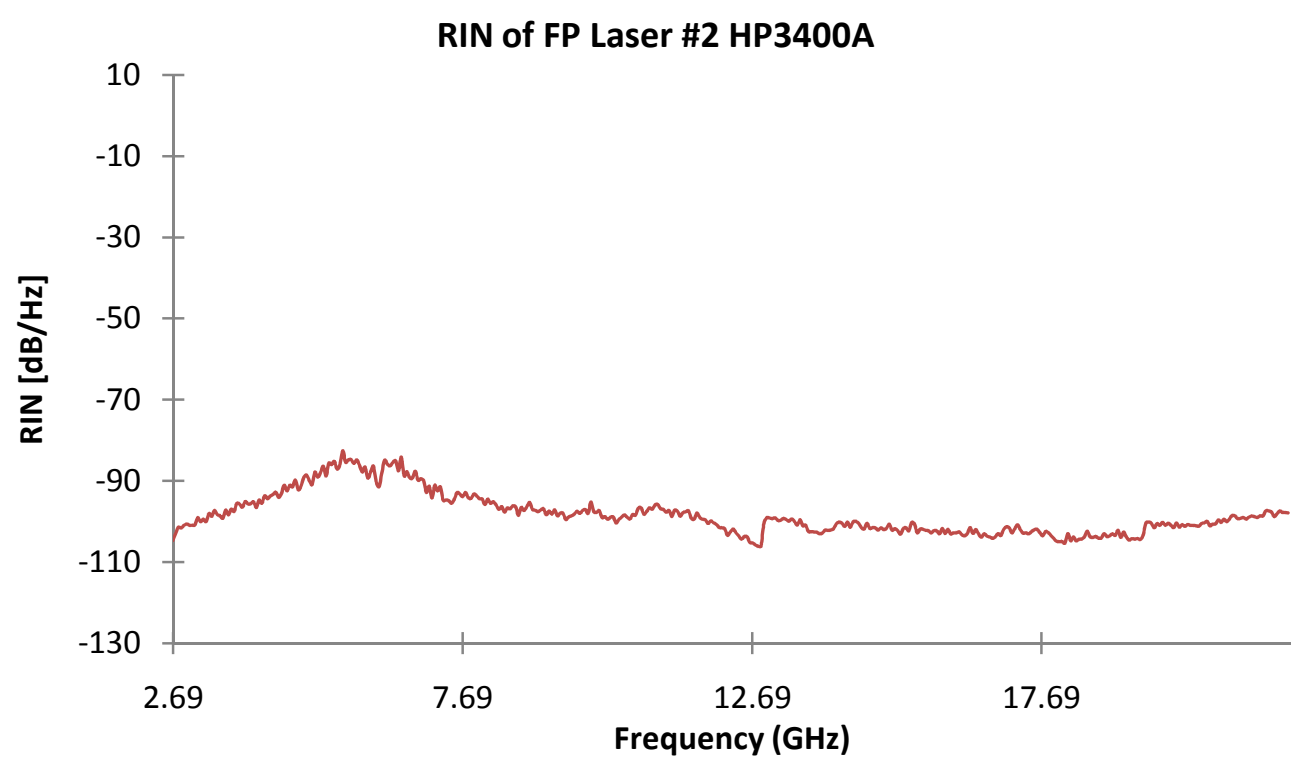

Figure 4-8: RIN of FP laser \#2 HP3400A at $G_{a 2}=15.5 \mathrm{~dB}$ 


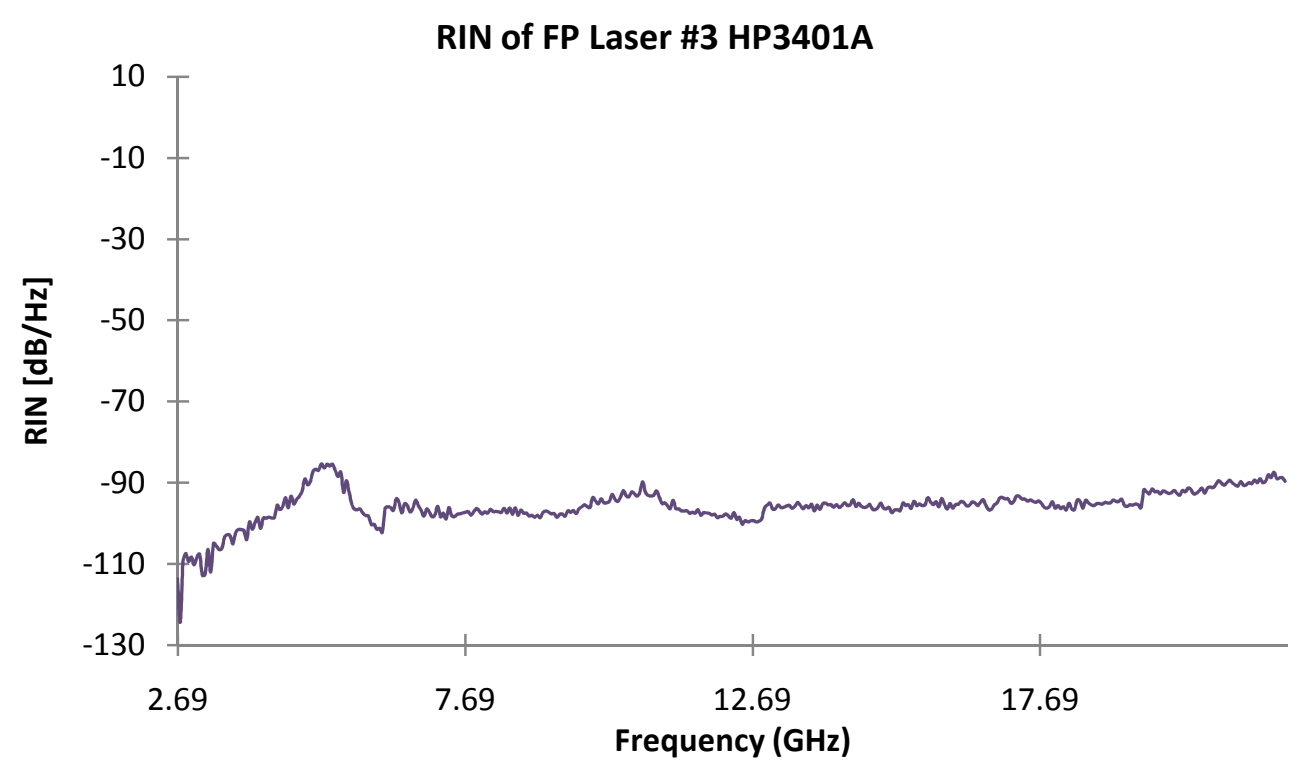

Figure 4-9: RIN of FP laser \#3 HP3401A at $G_{a 2}=15.5 \mathrm{~dB}$

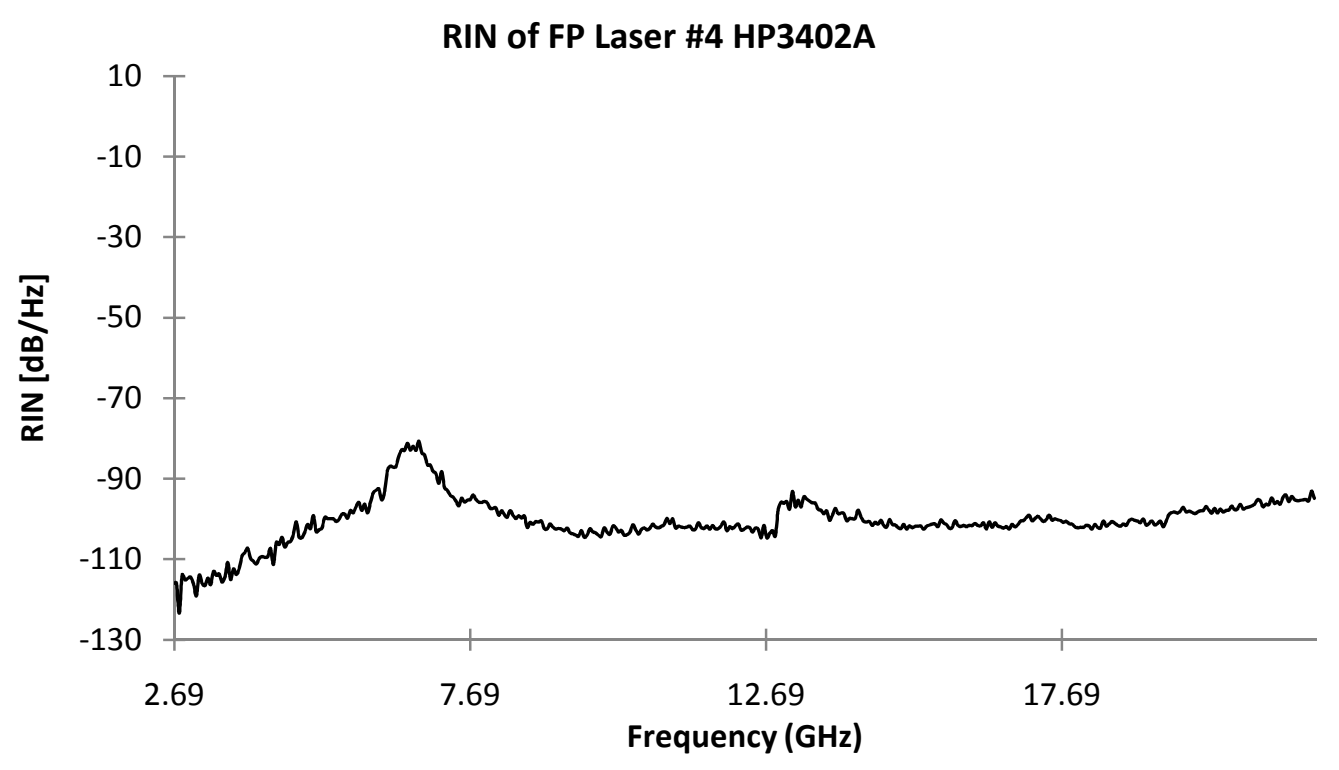

Figure 4-10: RIN of FP laser \#4 HP3402A at $G_{a 2}=15.5 \mathrm{~dB}$ 
The frequency responses of the New Focus 1422 broadband Amplifier and Picosecond Driver Amplifier 5865 from 0 to $20 \mathrm{GHz}$ that are used to amplify the Fabry-Pérot and DFB lasers and to calculate the RIN measurement.

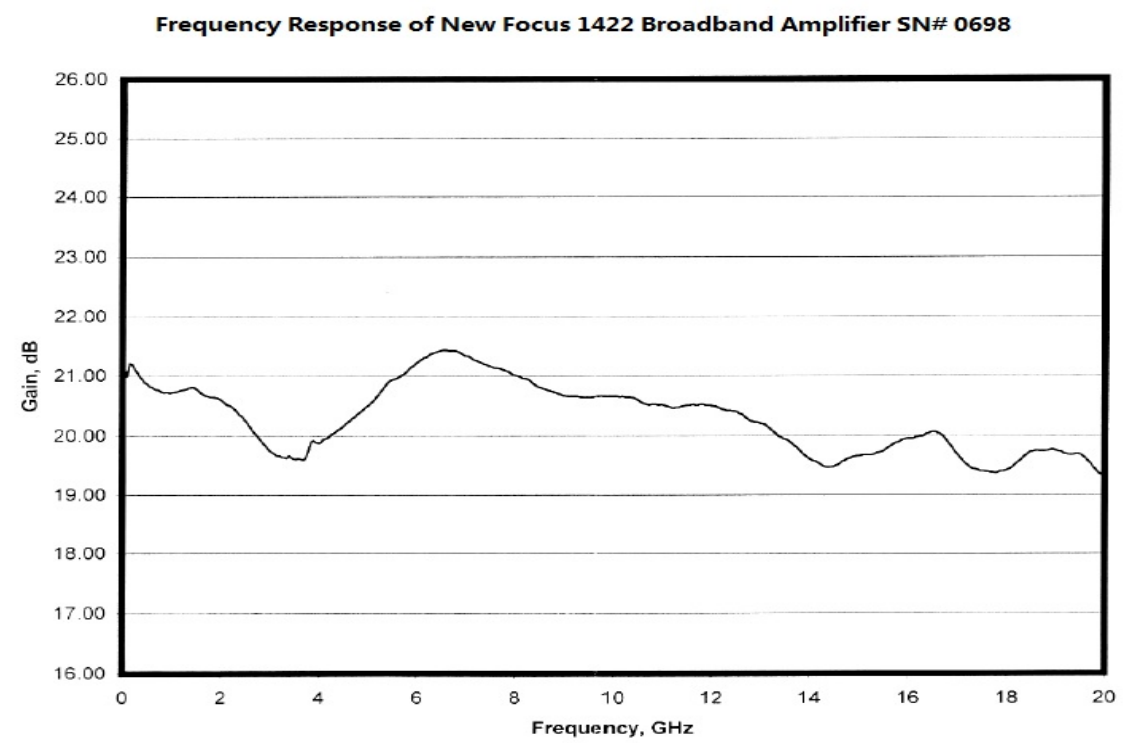

Figure 4-11: Frequency response of amplifier \#1

Picosecond Driver Amplifier 5865, S21 Magnitude (dB) Forward Gain

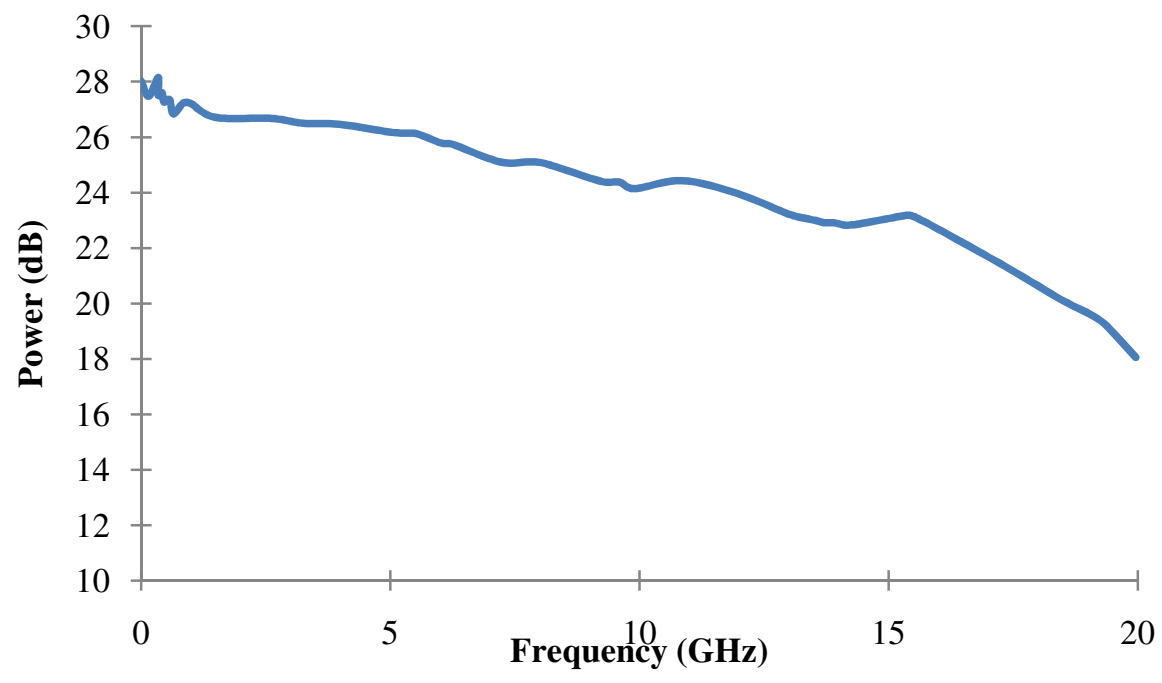

Figure 4-12: Frequency response of amplifier \#2 


\section{Chapter 5: Frequency Response of VCSEL under Optical}

\section{Pumping}

\subsection{Introduction of Optical Pumping (Injection Locking)}

This experiment is to investigate the bandwidth performance of the 850nm VCSELs with the optical pumping. However, the experiment of VCSEL injection locking is not successful. Theoretically, optical injection locking is able to improve laser performance both in digital and analog applications [12]. The large modulation bandwidth can be achieved with conventional semiconductor lasers at room temperature without using advanced devices and complex fabrication techniques [13]. Injection locking was first observed by Christiaan Huygens, who invented the pendulum clock. The two pendulum clocks with slightly different time synchronize perfectly when they are hanging on the same beam. The example of locking or synchronization can be observed in nature and daily lives. Synchronization can be witnessed in such models as the human biological clock to the sun rise and sun.

An injection-locked laser system contains two semiconductor lasers. The light from the source laser (master laser) is injected into the test laser (slave laser) oscillating above its threshold. The injected radiation competes with the spontaneous emission of the slave laser that is being amplified [14]. The slave laser will then emit the same optical frequency as the injected laser to achieve synchronization. A stable multi-wavelength master laser source can lock and improve transmitters at different channels 
simultaneously to reduce the cost of the transmitter modules. Therefore the multimode 850nm VCSELs are chosen to be the candidates for the master as well as the slave lasers.

\subsection{Experimental Setup of the Optical Pumping of VCSELs}

The experimental setup of the optical pumping for injection locking in VCSEL is shown in Figure 5-1. A current source, ILX Lightwave LDC-3744B, drives the master VCSEL current which is mounted on the LDM-4407 Temperature Controlled Laser Diode Mount. A second current controller, ILX Lightwave LDX-3207, is used to provide the DC biased signal for the test VCSEL. The reason for an additional laser diode controller for the test laser is to separate the control of the drive current for the Master laser in order to troubleshoot the experiment and also study the effects of the optical spectra and frequency responses when varying the drive current for each laser diode.

The slave VCSEL (test laser) which has to be DC biased to operate efficiently above the laser threshold at $1.8 \mathrm{~mA}$ before modulation, is mounted on the SMA connector to the inductor side of the bias $\mathrm{T}$ as shown in Figure 5-2 to output DC + RF signal. The Anritsu SMA cable is connected between the capacitor end of the Biased Tee and port 1 of the HP 8720B Network Analyzer to provide the RF modulated signal to the test laser. The second current controller LDX-3207 with a BNC to SMA converter is connected to the Biased Tee to provide the DC biased drive current for the test laser. In this experiment, the $\mathrm{DC}$ drive current is $10 \mathrm{~mA}$. 
The output of the test VCSEL is connected to the LC connector of the multimode fiber coupler with coupling ratios $50 \%-50 \%$ and configuration of $1 \times 2$. The coupler is by Phoenix Photonics which has a 3mm cable and optical fiber grated index of 62.5/125. The connectors of the coupler at the 50\%-50\% splitting ratio are LC and FC. The third connector is LC at $100 \%$ coupling ratio. The coupled laser light is then detected by the Newport Hi-Speed Photo-Detector D-15 which the optical signal is converted into electrical signal and to be amplified by the New Focus Broadband Amplifier 1422. The broadband amplifier 1422 is powered by New Focus +/- Volt Current-Limited Power Supply 901. The amplified signal is then sent to Port 2 of the HP 8720B Network Analyzer to obtain the frequency response. The frequency response is then recorded using LabVIEW.

Before obtaining the frequency response from injection locking, the effects of the optical spectra from the optical pump have to be observed. The optical spectra of the VCSELs need to have center wavelength close to each other to achieve the optimal locking [14]. 


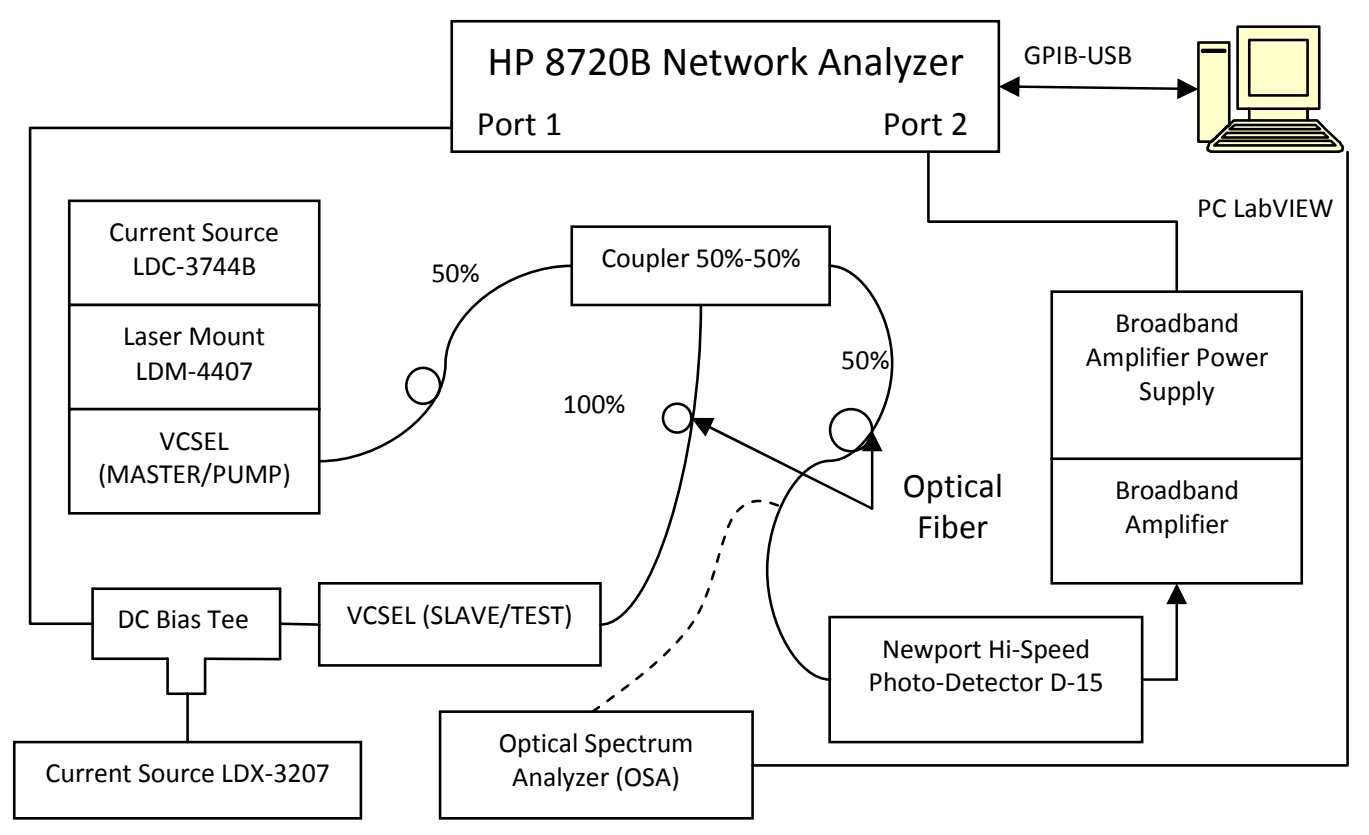

Figure 5-1: Experimental setup of injection locking for the 850nm VCSEL. Both the master and slave lasers are VCSELs

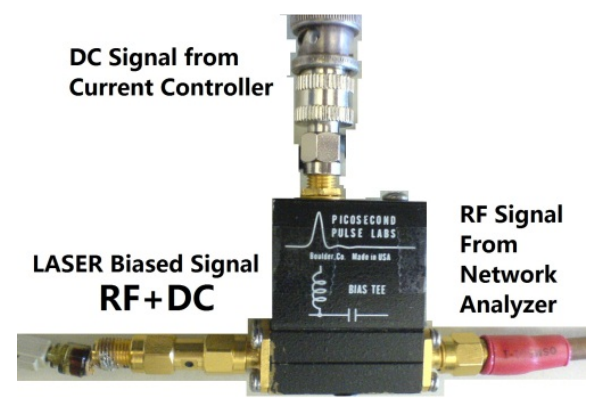

Figure 5-2: Biased Tee connection with RF signal from port 1 of HP 8720B Network Analyzer, DC biased signal from the Current Source ILX Lightwave LDX-3207 and the VCSEL with a modified pin is soldered onto the SMA connector outputting the DC biased, $R F+D C$ signal into the coupler 
There are three default-power VCSELs to choose for the experiment. The closest pair of VCSELs would be chosen as the master for pumping photons into the slave to perform injection locking.

The labels for the Default-Power VCSELs are:

1. VCSEL\#1 with Barrel: this is the VCSEL that is soldered on a SMA connector, namely barrel, for connecting to Bias Tee.

2. VCSEL\#2 without Barrel: this is the VCSEL that has three pins shorten to be directly connecting to the LDX-4407 Laser Diode Temperature Control Mount. It is used as Master Laser. The LC barrel is also cut out to prevent possible reflection from the laser using the coupler.

3. VCSEL\#3 Long Pins: this is the uncut VCSEL that can be soldered on the SMA connector or shorten to be connected to the LDX-4407.

The OSA of the VCSEL \#1 has to be compared with the VCSEL\#2 and VCSEL\#3 at various drive currents.

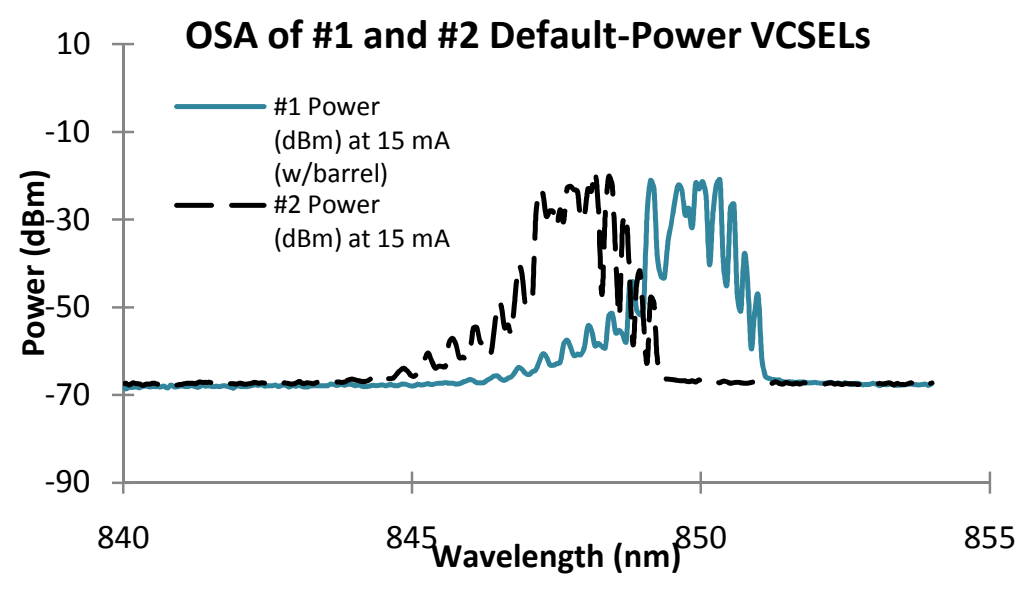

Figure 5-3: OSA plots of default-power VCSEL \#1 and \#2 
Figure 5-3 shows that the center wavelength is off by approximately $3 \mathrm{~nm}$ comparing between the VCSELs with and without the LC barrel with a drive current at $15 \mathrm{~mA}$. Next, VCSEL\#1 and \#3 are being compared together at 15mA in room temperature at $24{ }^{\circ} \mathrm{C}$.

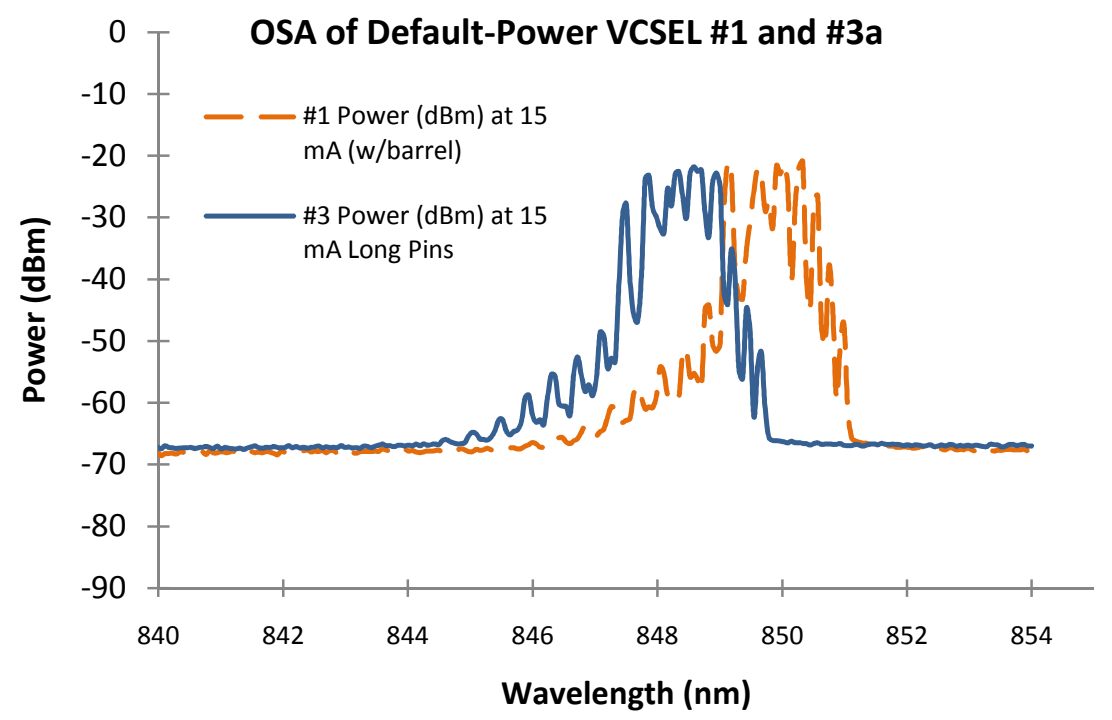

Figure 5-4: OSA plots of default-power VCSEL\#1 and \#3

The optical spectra of VCSEL \#1 and \#3 are pretty close to each other; therefore, VCSEL \#3 is chosen to be the master laser for optical pump, while VCSEL \#1 is chosen as the test laser. Next is to investigate the optical spectra when one laser is connected to the coupler with the other laser turned off. The differences in coupling ratio have been tested for the master and test lasers to ensure the coupler works accordingly. The four tests are:

1. Coupling the test laser to the $50 \%$ port of the coupler and outputting the signal at another $50 \%$ port of the coupler with the master laser turned off.

2. Coupling the test laser to the $100 \%$ port of the coupler and outputting the signal at the $50 \%$ port of the coupler with the master laser turned off. 
3. Coupling the master laser to the $50 \%$ port of the coupler and outpuittng the signal at another 50\% port of the coupler with the test laser turned off.

4. Coupling the master laser to the $100 \%$ port of the coupler and outputting the signal at the $50 \%$ port of the coupler with the test laser turned off.

Below are the optical spectra at $24{ }^{\circ} \mathrm{C}$ at various drive currents.

OSA of $850 \mathrm{~nm}$ Default-Pwr VCSEL Test at $50 \%$ Coupling with the Output at $50 \%$ at 24 C

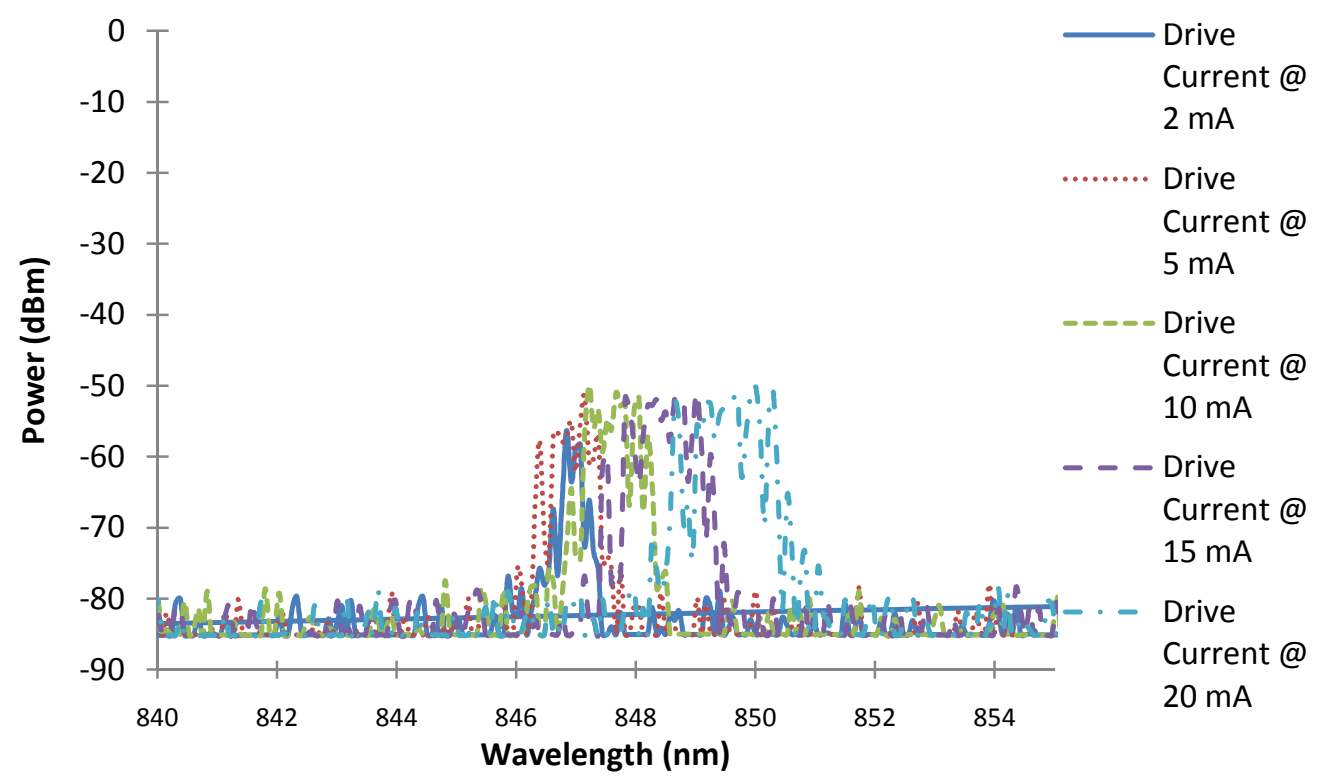

Figure 5-5: Test \#1: The optical spectra of the VCSELs from 2mA to 20mA. The average peak of the optical spectra is $-51.72 \mathrm{dBm}$ 


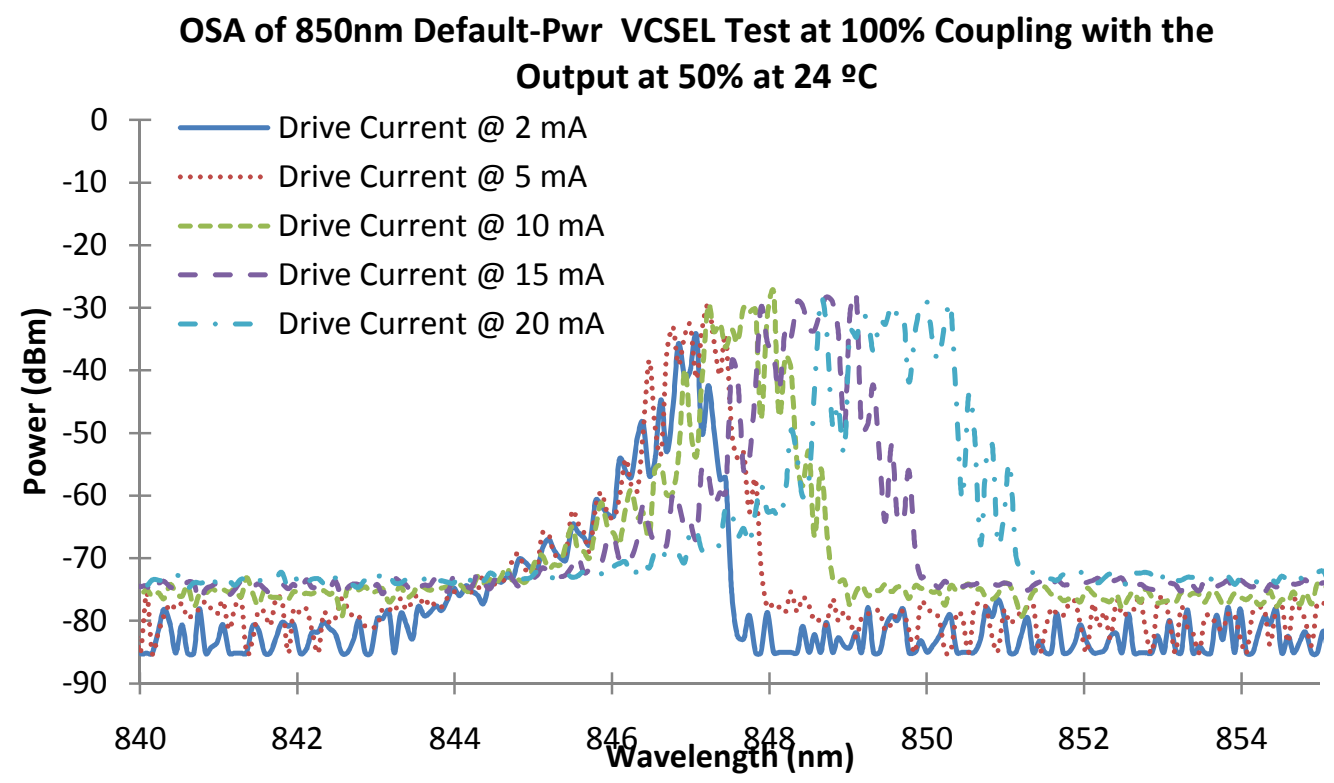

Figure 5-6: Test \#2: The optical spectra of the VCSELs from $2 m A$ to 20mA. The average peak of the optical spectra is $-29.552 \mathrm{dBm}$

OSA of 850 nm Default Power VCSELs Coupling w/ Master 50\% and Output $50 \%$ at 24 C

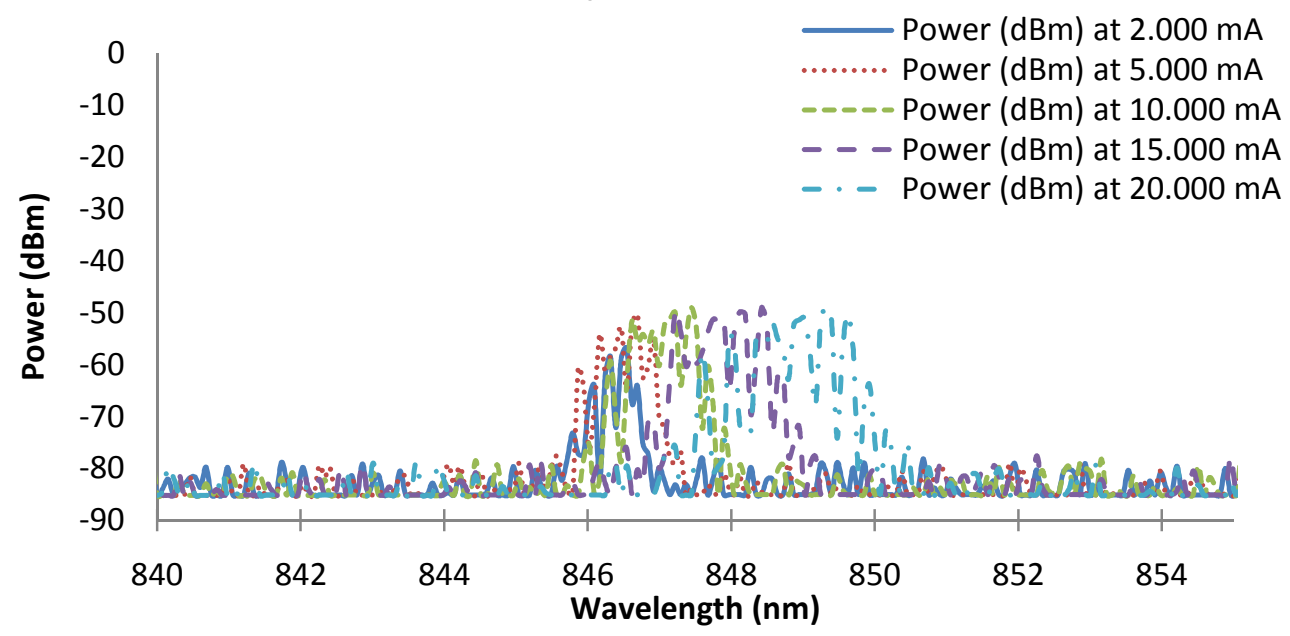

Figure 5-7: Test \#3: The optical spectra of the VCSELs from 2mA to 20mA. The average peak of the optical spectra is $-50.806 \mathrm{dBm}$ 


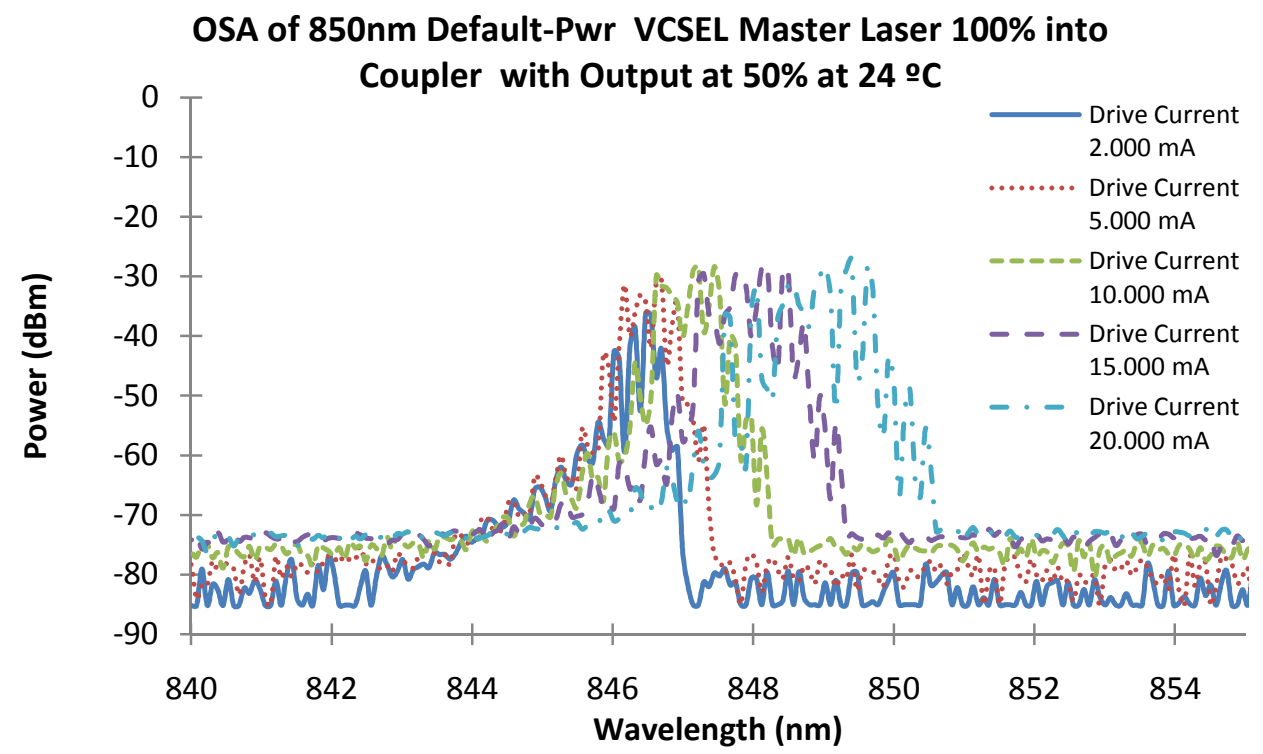

Figure 5-8: Test \#4: The optical spectra of the VCSELs from $2 \mathrm{~mA}$ to 20mA. The average peak of the optical spectra is $-30.084 \mathrm{dBm}$

Figures 5-5 and 5-7 indicate that the 50-50 coupler works as expected. The average peak magnitudes of the optical spectra of the test and master lasers that are passing through the $50 \%$ port of the coupler are $-51.72 \mathrm{dBm}$ and $-50.806 \mathrm{dBm}$ respectively. The average peak magnitudes from the $50 \%$ port are lower than those of the test and master lasers that are passing through the $100 \%$ port of the coupler in Figures 5-6 and 5-8 at $-29.552 \mathrm{dBm}$ and $-30.084 \mathrm{dBm}$ respectively. After the coupler has been tested, the next step is to try the optical pumping by coupling the master and test lasers together.

According to the block diagram in Figure 5-1, each laser diode has to be controlled by a separately current source. Since the master and slave VCSELs have the same center wavelengths at $850 \mathrm{~nm}$, both laser need to be controlled at the same drive current increments: $2 \mathrm{~mA}, 5 \mathrm{~mA}, 10 \mathrm{~mA}, 15 \mathrm{~mA}$, and $20 \mathrm{~mA}$. 
There are two results by coupling the master and test lasers at different coupling ratios:

1. Coupling the master laser at the $100 \%$ port with the test laser at the $50 \%$ port.

2. Coupling the master laser at the $50 \%$ port with the test laser at the $100 \%$ port.

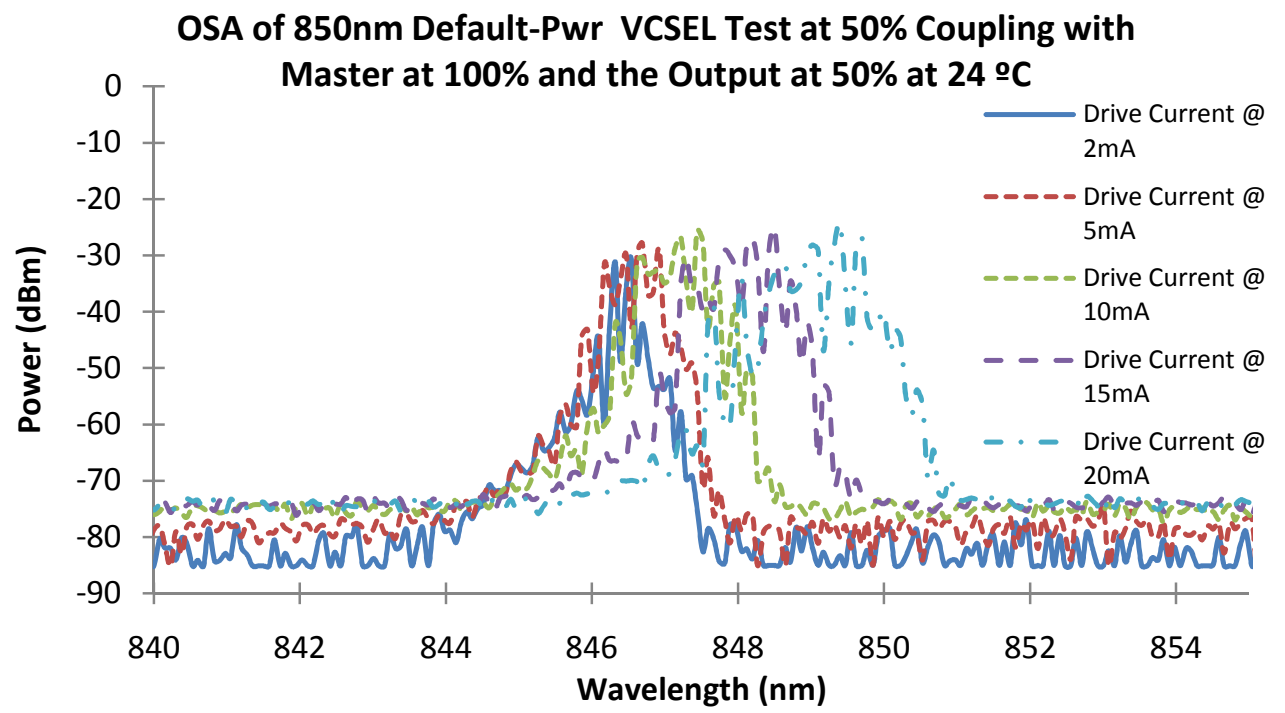

Figure 5-9: Test \#1: The optical spectra of the VCSELs after optical pumping from $2 \mathrm{~mA}$ to $20 \mathrm{~mA}$. The average peak of the optical spectrum is $-26.664 \mathrm{dBm}$

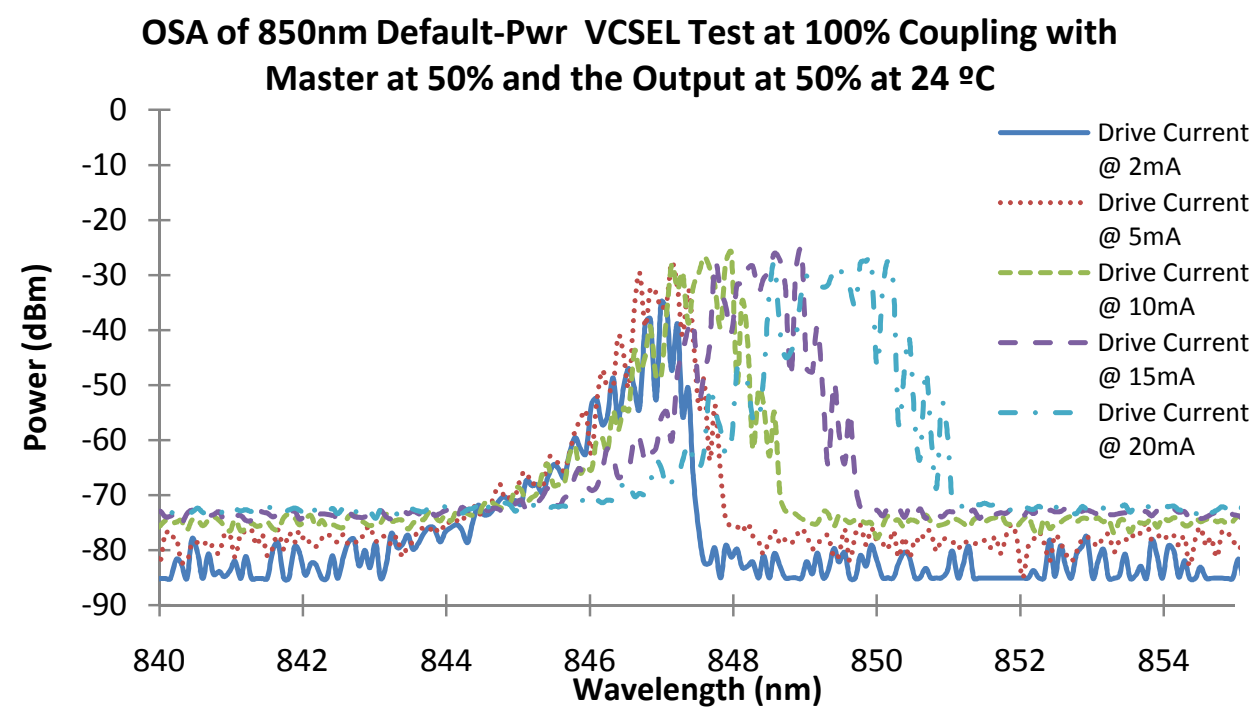

Figure 5-10: Test \#2: The optical spectra of the VCSELs after optical pumping from $2 \mathrm{~mA}$ to $20 \mathrm{~mA}$. The average peak of the optical spectrum is $-28.164 \mathrm{dBm}$ 
The optical pumping spectra are very similar to each other between test \#1 and \#2. The optical spectrum magnitudes are $-26.664 \mathrm{dBm}$ and $-28.164 \mathrm{dBm}$ for test \#1 and test \#2 respectively. They are similar to the spectra that are measured independently without coupling as shown in Figure 2-25 and 2-26. The frequency response of the optical pumping is also being tested.

In the optical pumping experiment, there is a possibility that the test laser which is connected to the $100 \%$ port of the coupler is able to show the frequency responses. On the other hand, if the test laser is connected to the $50 \%$ port, the frequency responses become very noisy. This phenomenon is being observed according to the following tests:

1. The test and master lasers are coupling with the $50 \%$ and $100 \%$ ports of the coupler respectively with an output at the second $50 \%$ port.

2. The test laser is coupled with the $50 \%$ port of the coupler with an output at the $50 \%$ port. The master laser is turned off.

3. The test and master lasers are coupling with the $100 \%$ and $50 \%$ ports of the coupler respectively with an output at the second $50 \%$ port.

4. The test laser is coupled with the $100 \%$ port of the coupler with an output at the $50 \%$ port. The master laser is turned off. 


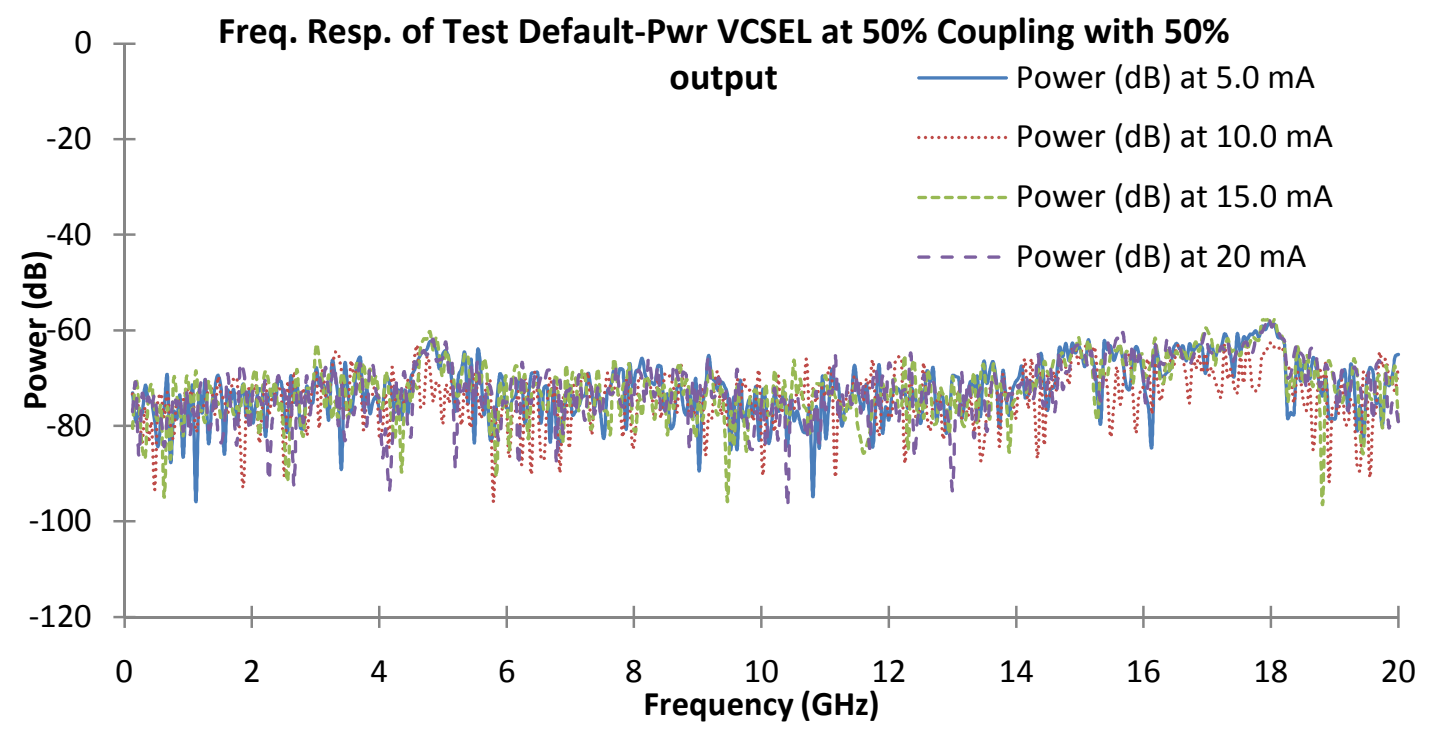

Figure 5-11: Test \#1: The frequency responses are noisy when the test laser is connected to the 50\% port with master laser turned off.

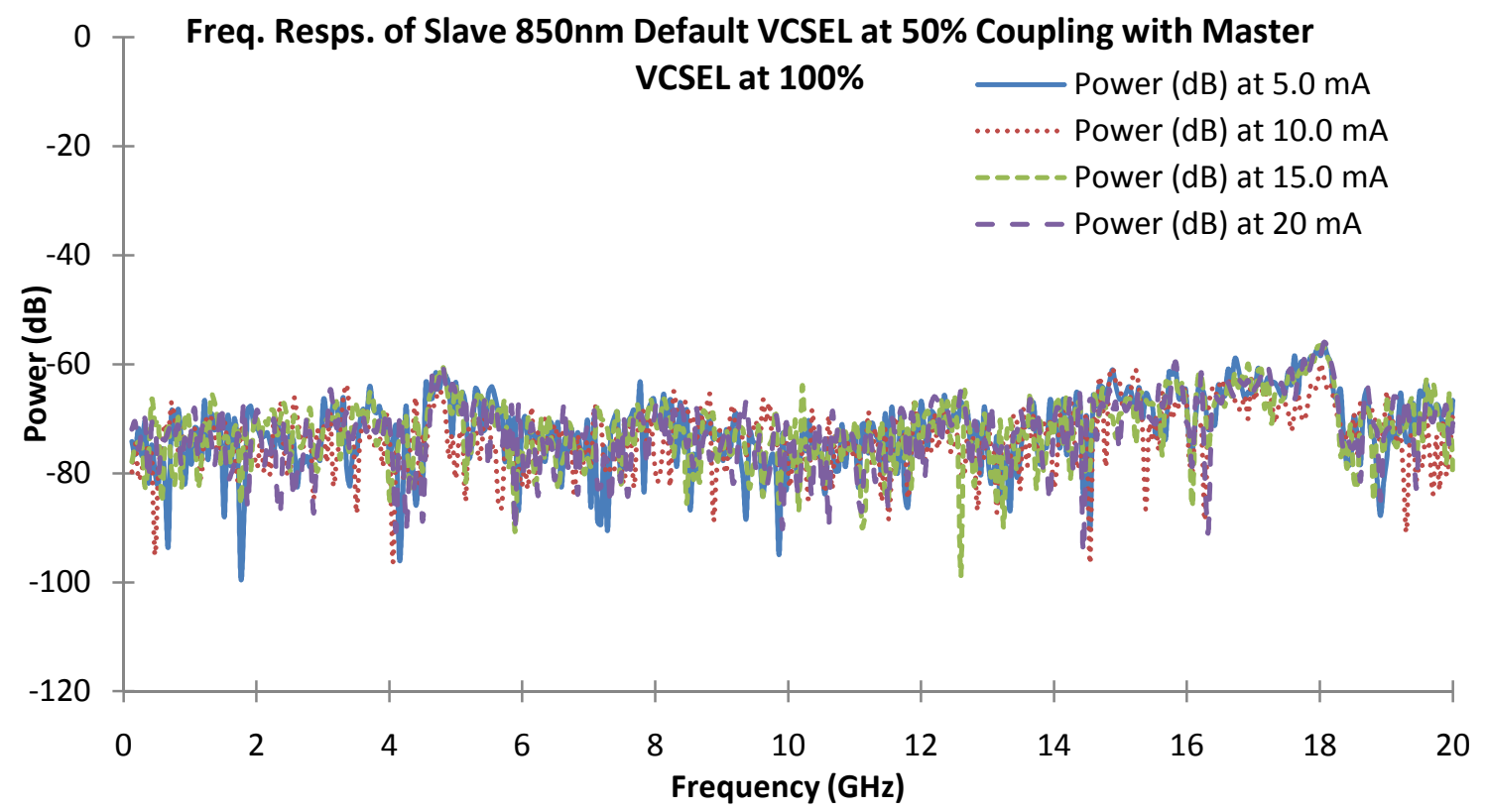

Figure 5-12: Test \#2: The frequency responses are noisy when the test laser is connected to the 50\% port with the master laser at the $100 \%$ port of the coupler 


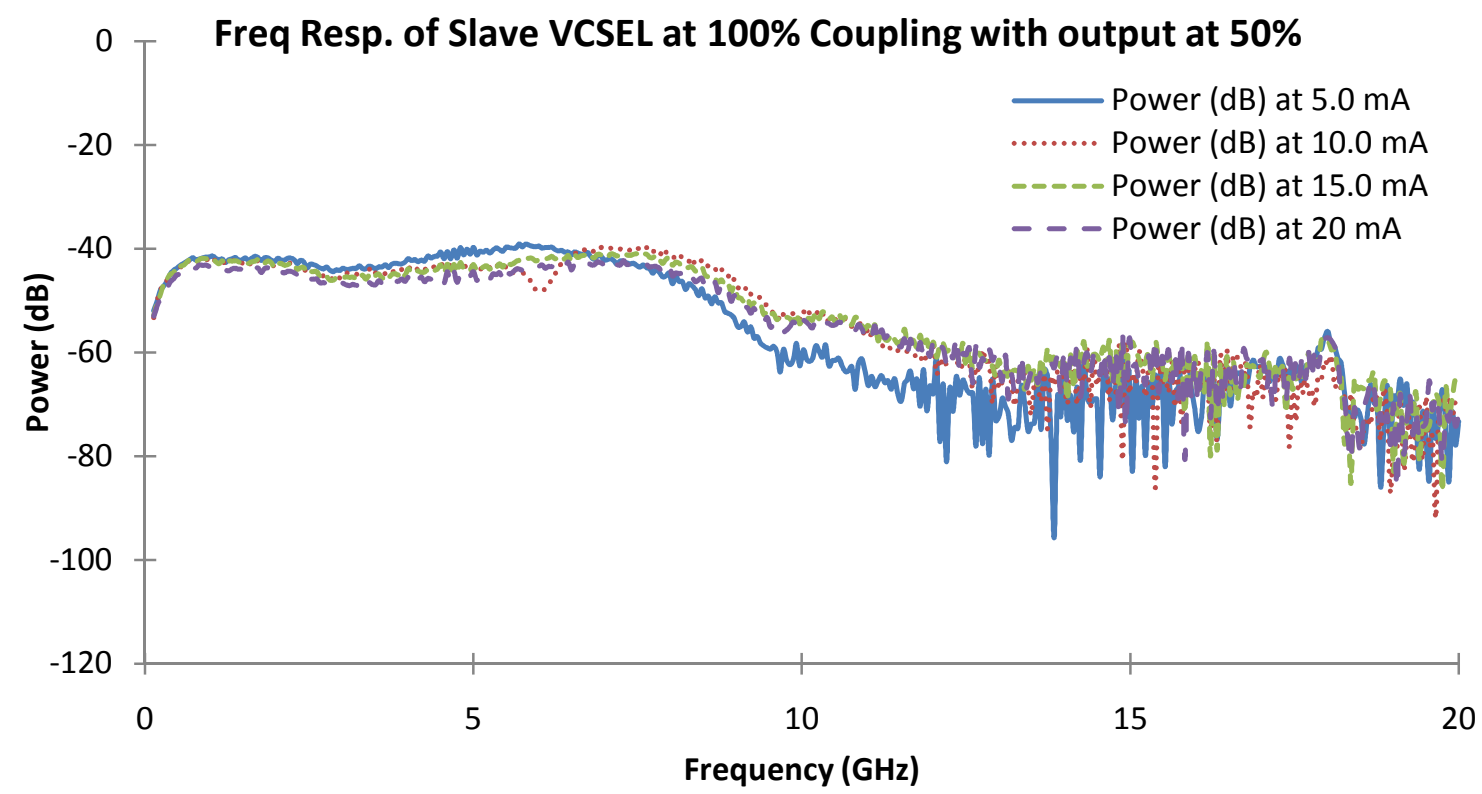

Figure 5-13: Test \#3: The frequency responses are visible when the test laser is connected to the 100\% port coupling with the output at the 50\% while the master laser is turned off.

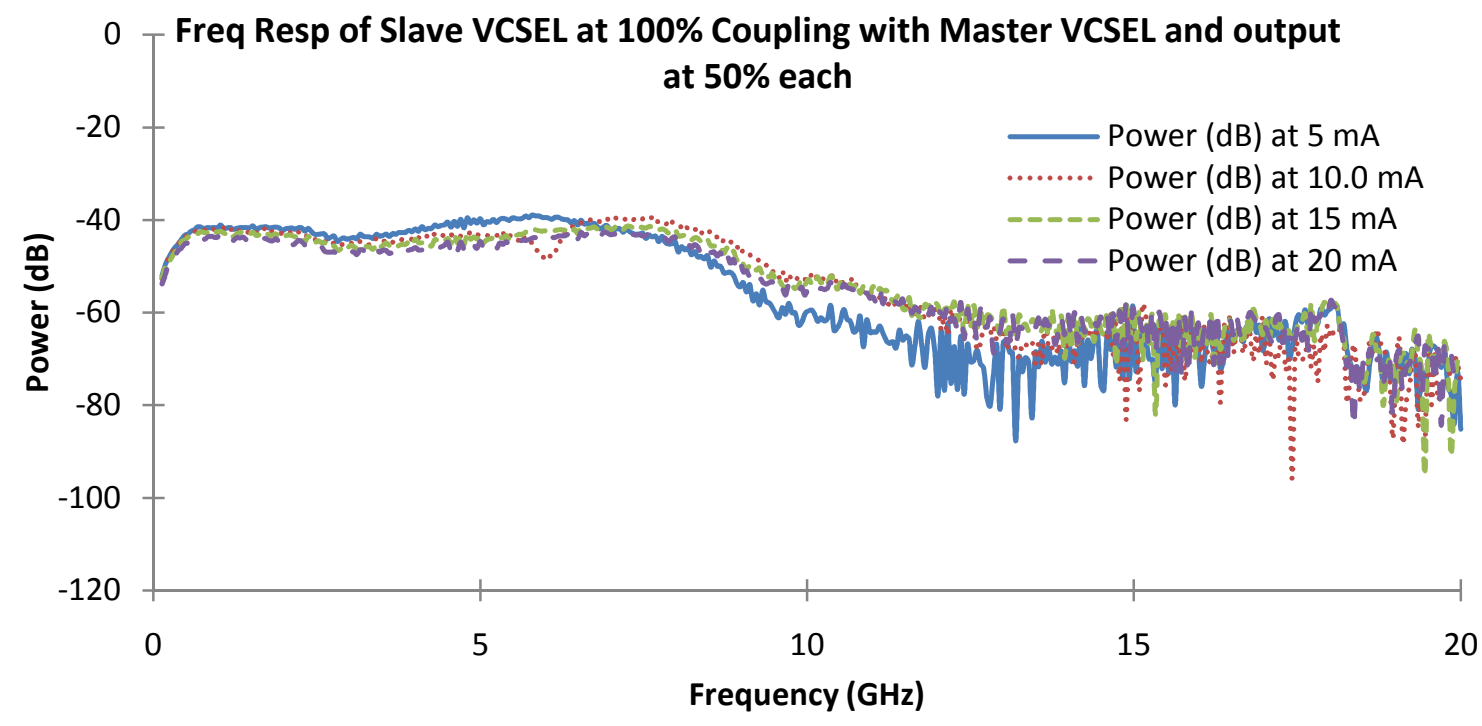

Figure 5-14: Test \#4: The frequency responses are visible when the test laser is connected to the 100\% port with the master laser turned on at the 50\% port. 
There are almost no differences between the frequency responses in Figure 5-13 and 514. The VCSEL has high reflecting mirrors, which is very difficult for injection locking to occur from the master laser to the slave laser. Figure 5-11 and 5-12 have only the test instrument noise. Therefore, Figure 5-11 to 5-14 show no sign of optical pump or injection locking. 


\section{Chapter 6: Conclusions and Future Works}

The experimental setups that I have developed are later used in JDSU projects and have brought Cal Poly San Louis Obispo new sponsor projects and donations from JDSU.

The laser DC measurement experiment indicates that the optical powers of the lasers are temperature dependent. The results are being observed in the VCSELs and DFB. As the temperature increases, the threshold current that drives the optical power decreases and the optical spectrum shifts from the left to the right towards a longer center wavelength. This is because more drive current is needed to compensate the loss of the electron-hole recombination due to thermal vibration to increase the photon gain to achieve stimulated emission. Only the optical spectrum of the Fabry-Pérot laser has been tested and recorded for DC measurement due the limitation in hardware of the FP laser lacking a temperature controlling unit.

The frequency responses of the lasers are derived from the rate equations. The rate equations are combinations of the carrier and photon density rates which are used to derive the resonance frequencies. These resonance frequencies are then used to simulate the frequency responses of the DFB laser across different output powers at $20^{\circ} \mathrm{C}$. In the experiment, three power levels of VCSELs and Fabry-Pérot lasers have been tested for the frequency responses at different drive currents. The VCSEL with default power has a 
bandwidth of $9 \mathrm{GHz}$, while the low power is $9.17 \mathrm{GHz}$, and the high power is $7.78 \mathrm{GHz}$. The four Fabry-Pérot lasers that are being tested have bandwidths: $3.16 \mathrm{GHz}, 3.31 \mathrm{GHz}$, $5.05 \mathrm{GHz}$, and $7.88 \mathrm{GHz}$.

The intrinsic relative intensity noises (RINs) of the DFB and FP lasers have been extracted. The equations of the parasitic noises such as shot and thermal have to be eliminated in order to extract the lasers' RINs. The RIN of the DFB laser decreases from -32 to $-107 \mathrm{~dB} / \mathrm{Hz}$ when the drive current increases from $5 \mathrm{~mA}$ to $48 \mathrm{~mA}$. Since there is no controller to alter the drive current of the FP laser, there is only one RIN for each FP laser. In the experiment, the RINs results of the FP lasers are physically unrealistic when the gain of the Picosecond Pulse Labs 5865 amplifier is $26.5 \mathrm{~dB}$. This is because the RINs that are being measured are less than the total parasitic noises (shot noise + thermal noise). Further investigation is recommended to obtain the RIN of the FP lasers.

The optical pumping experiment using 850nm VCSEL is investigated. Since the frequency responses are still the same regardless whether the master laser is on or off while coupling with the slave laser, the optical pumping experiment is not successful. The VCSELs have high reflecting mirrors, which make the master laser very hard to optically pump or injection lock the slave laser. 


\section{APPENDIX A: Equipment Lists}

\section{VCSEL DC LVI Curves Measurement:}

\begin{tabular}{|c|c|c|c|c|c|}
\hline \# & Brand & Description & Model \# & Serial \# & INFO \\
\hline 1 & Honeywell & VCSEL, 4.25Gbps $850 \mathrm{~nm}$ & HFE4192-901 & N/A & $\begin{array}{c}\text { Laser: } \\
\text { VCSEL (LC } \\
\text { TOSA) }\end{array}$ \\
\hline 2 & $\begin{array}{c}\text { ILX } \\
\text { Lightwave }\end{array}$ & $\begin{array}{c}\text { Laser Diode Current and Temperature } \\
\text { Controller }\end{array}$ & LDC-3744B & EE6573 & \\
\hline 3 & $\begin{array}{c}\text { ILX } \\
\text { Lightwave }\end{array}$ & Temperature Controlled Laser Diode Mount & LDM-4407 & $\begin{array}{l}44071645 \\
\text { DL983391 }\end{array}$ & $1 / 13 / 2005$ \\
\hline 4 & Newport & Photo Detector & 818-SL & 1586 & $400-1100 \mathrm{~nm}$ \\
\hline 5 & FIS & $\begin{array}{l}\text { Optical Fiber OFNR RISER CABLE 083810FT } \\
5143 \text { E207090(UL) C(UL), 62.5/125 }\end{array}$ & S27ULU2fis & N/A & $\begin{array}{c}\text { Multi Mode, } \\
\text { LC/FC } \\
\text { Connector }\end{array}$ \\
\hline 6 & $\begin{array}{l}\text { National } \\
\text { Instruments }\end{array}$ & GPIB to Hi-Speed USB converter & GPIB-USB-HS & 11B4F84 & $\begin{array}{c}\text { Work } \\
\text { together with } \\
\text { LabVIEW }\end{array}$ \\
\hline 7 & $\begin{array}{l}\text { National } \\
\text { Instruments }\end{array}$ & $\begin{array}{l}\text { LabView } 7.1 \text { captures the data from } \\
\text { measurement instruments and plot graphs in PC }\end{array}$ & LabView 7.1 & & $\begin{array}{c}\text { Software: } \\
\text { Control } \\
\text { Equipments }\end{array}$ \\
\hline 8 & SONY VAIO & Windows XP Pro Service Pack 2 & S260P & & $\begin{array}{c}\text { Run LabView } \\
7.0\end{array}$ \\
\hline
\end{tabular}

\section{DFB Laser DC LVI Curves Measurement:}

\begin{tabular}{|c|c|c|c|c|c|}
\hline \# & Brand & Description & Model \# & Serial \# & INFO \\
\hline 1 & $\begin{array}{c}\text { Seastar } \\
\text { Optics Inc. }\end{array}$ & Distributed Feedback Lasers (DFB) & $\begin{array}{c}\text { PM-5501A- } \\
1550\end{array}$ & SP4997 & $\begin{array}{l}\text { Laser: } \\
\text { ML974A2F }\end{array}$ \\
\hline 2 & Kette & LD Driver \& Temperature Controller & KNN-2000LT & EE6574 & $\begin{array}{c}\text { Temperature } \\
\text { Control Not } \\
\text { working }\end{array}$ \\
\hline 3 & Newport & Photo Detector & 818-SL & 1586 & $400-1100 \mathrm{~nm}$ \\
\hline 4 & Newport & $\begin{array}{c}\text { COLLIMATOR FIBER OPTIC OPTION, } 1550 \\
n \mathrm{~nm}, 50 / 125 \text { Multimode }\end{array}$ & F-COL-50-15-OPT & N/A & $\begin{array}{l}\text { 1550nm, } 50 / 125 \\
\text { Multi-mode }\end{array}$ \\
\hline 7 & SONY VAIO & Windows XP Pro Service Pack 2 & S260P & N/A & Run LabView 7.1 \\
\hline
\end{tabular}


VCSEL Laser Optical Spectrum Measurement:

\begin{tabular}{|c|c|c|c|c|c|}
\hline \# & Brand & Description & Model \# & Serial \# & INFO \\
\hline 1 & Honeywell & VCSEL, 4.25Gbps $850 \mathrm{~nm}$ & HFE4192-901 & N/A & $\begin{array}{c}\text { Laser: } \\
\text { VCSEL (LC } \\
\text { TOSA) }\end{array}$ \\
\hline 2 & $\begin{array}{c}\text { ILX } \\
\text { Lightwave }\end{array}$ & Laser Diode Current and Temperature Controller & LDC-3744B & 37443707 & \\
\hline 3 & $\begin{array}{c}\text { ILX } \\
\text { Lightwave }\end{array}$ & Temperature Controlled Laser Diode Mount & LDM-4407 & $\begin{array}{l}44071645 \\
\text { DL983391 }\end{array}$ & $1 / 13 / 2005$ \\
\hline 4 & $\mathrm{HP}$ & Optical Spectrum Analyzer & HP 71450A & EE 5165 & \\
\hline 5 & FIS & $\begin{array}{c}\text { Optical Fiber OFNR RISER CABLE 083810FT } 5143 \\
\text { E207090(UL) C(UL), 62.5/125 }\end{array}$ & S27ULU2fis & N/A & $\begin{array}{c}\text { Multi-Mode, } \\
\text { LC/FC } \\
\text { Connector }\end{array}$ \\
\hline 6 & $\begin{array}{c}\text { National } \\
\text { Instruments }\end{array}$ & GPIB to Hi-Speed USB converter & GPIB-USB-HS & 11B4F84 & \\
\hline 7 & $\begin{array}{l}\text { National } \\
\text { Instruments }\end{array}$ & $\begin{array}{c}\text { LabView } 7.1 \text { captures the data from measurement } \\
\text { instruments and plot graphs in PC }\end{array}$ & LabView 7.1 & & $\begin{array}{c}\text { Software: } \\
\text { Control } \\
\text { Equipment }\end{array}$ \\
\hline 8 & SONY VAIO & Windows XP Pro Service Pack 2 & S260P & & $\begin{array}{c}\text { Run LabView } \\
7.1\end{array}$ \\
\hline
\end{tabular}

\begin{tabular}{|r|c|}
\hline VCSEL and DFB OSA Settings & \\
\hline Resolution Bandwidth (nm): & 0.08 \\
\hline Span (nm): & 10 \\
\hline Sensitivity (dBm): & -75 \\
\hline
\end{tabular}




\section{DFB Laser Optical Spectrum Measurement:}

\begin{tabular}{|c|c|c|c|c|c|}
\hline \# & Brand & Description & Model \# & Serial \# & INFO \\
\hline 1 & Kette & LD Driver \& Temperature Controller & KNN-2000LT & EE6574 & $\begin{array}{c}\text { Temperature } \\
\text { Control not } \\
\text { working }\end{array}$ \\
\hline 2 & $\begin{array}{l}\text { Seastar } \\
\text { Optics } \\
\text { Inc. }\end{array}$ & Distributed Feedback Lasers (DFB) & PM-5501A-1550 & SP4997 & $\begin{array}{c}\text { Laser: } \\
\text { ML974A2F }\end{array}$ \\
\hline 3 & $\mathrm{HP}$ & Optical Spectrum Analyzer & $71450 \mathrm{~A}$ & EE5624, EE5625 & $\begin{array}{c}\text { Display + } \\
\text { OSA }\end{array}$ \\
\hline 4 & FIS & $\begin{array}{c}\text { Optical Fiber OFNR RISER CABLE } \\
\text { 083810FT 5083 E207090(UL) C(UL), } \\
\text { SMF28E, 9/125 }\end{array}$ & SMF28E & N/A & $\begin{array}{l}\text { Single Mode, } \\
\text { FC Connector }\end{array}$ \\
\hline 5 & $\begin{array}{c}\text { National } \\
\text { Instruments }\end{array}$ & $\begin{array}{c}\text { LabView } 7.1 \text { captures the data from } \\
\text { measurement instruments and plot } \\
\text { graphs in PC }\end{array}$ & LabView 7.1 & & $\begin{array}{l}\text { Software: } \\
\text { Control } \\
\text { Equipment }\end{array}$ \\
\hline 6 & $\begin{array}{l}\text { SONY } \\
\text { VAIO }\end{array}$ & Windows XP Pro Service Pack 2 & S260P & & $\begin{array}{c}\text { Run LabView } \\
7.1\end{array}$ \\
\hline
\end{tabular}

Fabry-Pérot Lasers Optical Spectrum Measurement:

\begin{tabular}{|c|c|c|c|c|c|}
\hline \# & Brand & Description & Model \# & Serial \# & INFO \\
\hline 1 & Power One & FP Laser Power Supply & HCBB-75W-A & N/A & \\
\hline 2 & HP & Fabry-Pérot Lasers & $\begin{array}{c}\# 1 \& \# 2 \\
\text { HP83400A x } 2 \\
\text { \#3 HP83401A } \\
\# 4 \text { HP83402A }\end{array}$ & $\begin{array}{l}2850 \mathrm{~A} 00108 \\
2860 \mathrm{~A} 00114 \\
2860 \mathrm{~A} 00108 \\
2826 \mathrm{~A} 00203\end{array}$ & $\begin{array}{c}\text { \#1 \& \#2 SMF } \\
\text { \#3 MMF } \\
\text { \#4 Unknown }\end{array}$ \\
\hline 3 & HP & Optical Spectrum Analyzer & $71450 \mathrm{~A}$ & $\begin{array}{l}\text { EE5624, } \\
\text { EE5625 }\end{array}$ & Display + OSA \\
\hline 4 & FIS & $\begin{array}{l}\text { Optical Fiber OFNR RISER CABLE 083810FT } \\
5083 \text { E207090(UL) C(UL), SMF28E, 9/125 }\end{array}$ & SMF28E & N/A & $\begin{array}{l}\text { Single Mode, FC } \\
\text { Connector }\end{array}$ \\
\hline 5 & $\begin{array}{l}\text { National } \\
\text { Instruments }\end{array}$ & $\begin{array}{l}\text { LabView } 7.1 \text { captures the data from } \\
\text { measurement instruments and plot graphs in PC }\end{array}$ & LabView 7.1 & & $\begin{array}{l}\text { Software: Control } \\
\text { Equipment }\end{array}$ \\
\hline 6 & SONY VAIO & Windows XP Pro Service Pack 2 & S260P & & Run LabView 7.1 \\
\hline
\end{tabular}




\begin{tabular}{|r|c|c|c|c|}
\cline { 2 - 5 } \multicolumn{1}{|c|}{ Fabry Parot Laser OSA settings } & $\# 1$ & $\# \mathbf{2}$ & $\mathbf{\# 3}$ & $\mathbf{\# 4}$ \\
\hline Resolution Bandwidth (nm): & 0.5 & 0.5 & 0.5 & 0.5 \\
\hline Span (nm): & 50 & 50 & 50 & 40 \\
\hline Sensitivity (dBm): & -52.82 & -52.82 & -52.82 & -52.74 \\
\hline
\end{tabular}

\section{VCSELs Bandwidths:}

\begin{tabular}{|c|c|c|c|c|c|}
\hline \# & Brand & Description & Model \# & Serial \# & INFO \\
\hline 1 & Honeywell & VCSEL, 4.25Gbps 850nm & HFE4192-901 & & $\begin{array}{l}\text { Laser: VCSEL (LC } \\
\text { TOSA) }\end{array}$ \\
\hline 2 & ILX Lightwave & Precision Current Source & LDX-3207 & 860815 & $8 / 9 / 1986$ \\
\hline 3 & New Focus & +/- 15 Volt Current-Limited Power Supply & 901 & & $0503-10382$ \\
\hline 4 & New Focus & Broadband Amplifier & 1422 & & \\
\hline 5 & Newport & Photo Detector & $\mathrm{D}-15 \mathrm{FC}$ & 1472 & \\
\hline 6 & FIS & $\begin{array}{c}\text { Optical Fiber OFNR RISER CABLE 083810FT } 5143 \\
\text { E207090(UL) C(UL), 62.5/125 }\end{array}$ & S27ULU2fis & $\mathrm{N} / \mathrm{A}$ & $\begin{array}{l}\text { Multi Mode, LC/FC } \\
\text { Connector }\end{array}$ \\
\hline 7 & $\begin{array}{l}\text { Picosecond Pulse } \\
\text { Labs }\end{array}$ & Bias Tee & $5541 \mathrm{~A}$ & $\begin{array}{l}596 \\
8 / 97\end{array}$ & 26Ghz, 8ps \\
\hline 8 & Anritsu & SMA Cable & $3670 \mathrm{~K} 50-2$ & & SC3855 \\
\hline 9 & & SMA Connector 2-sided & & & \\
\hline 10 & & SMA to BNC connector & & & \\
\hline 11 & HP & Network Analyzer & $8720 \mathrm{~B}$ & EE5165 & $130 \mathrm{MHz}-20 \mathrm{GHz}$ \\
\hline 12 & HP & Calibration Kit $2.5 \mathrm{~mm}$ Economy Calibration Kit & $85052 \mathrm{D}$ & & \\
\hline 13 & Fluke & Digital Multi Meter & 87 & EE5710 & \\
\hline 14 & $\begin{array}{c}\text { National } \\
\text { Instruments }\end{array}$ & GPIB to Hi-Speed USB converter & $\begin{array}{l}\text { GPIB-USB- } \\
\text { HS }\end{array}$ & 11B4F84 & \\
\hline 15 & $\begin{array}{c}\text { National } \\
\text { Instruments }\end{array}$ & $\begin{array}{c}\text { LabView } 7.0 \text { captures the data from measurement instruments } \\
\text { and plot graphs in PC }\end{array}$ & LabView 7.0 & & $\begin{array}{l}\text { Software: Control } \\
\text { Equipment }\end{array}$ \\
\hline 16 & SONY VAIO & Windows XP Pro Service Pack 2 & S260P & & Run LabView 7.0 \\
\hline
\end{tabular}


Fabry-Pérot Laser Bandwidths:

\begin{tabular}{|c|c|c|c|c|c|}
\hline$\#$ & Brand & Description & Model \# & Serial \# & INFO \\
\hline 1 & Power One & FP Laser Power Supply & HCBB-75W-A & N/A & \\
\hline 2 & HP & Fabry-Pérot Lasers & $\begin{array}{c}\# 1 \& \text { \#2 HP83400A } \\
\text { x } 2 \\
\text { \#3 HP83401A } \\
\text { \#4 HP83402A }\end{array}$ & $\begin{array}{l}\text { 2850A00108 } \\
\text { 2860A00114 } \\
\text { 2860A00108 } \\
\text { 2826A00203 }\end{array}$ & $\begin{array}{c}\text { \#1 \& \#2 SMF } \\
\text { \#3 MMF } \\
\text { \#4 Unknown }\end{array}$ \\
\hline 3 & HP & Optical Spectrum Analyzer & $71450 \mathrm{~A}$ & $\begin{array}{l}\text { EE5624, } \\
\text { EE5625 }\end{array}$ & Display + OSA \\
\hline 4 & FIS & $\begin{array}{c}\text { Optical Fiber OFNR RISER CABLE } \\
\text { 083810FT 5083 E207090(UL) C(UL), } \\
\text { SMF28E, 9/125 }\end{array}$ & SMF28E & N/A & $\begin{array}{l}\text { Single Mode, FC } \\
\text { Connector }\end{array}$ \\
\hline 5 & HP & Network Analyzer & $8720 \mathrm{~B}$ & EE5165 & $\begin{array}{c}130 \mathrm{MHz}-20 \\
\mathrm{GHz}\end{array}$ \\
\hline 6 & HP & $\begin{array}{l}\text { Calibration Kit 2.5mm Economy Calibration } \\
\text { Kit }\end{array}$ & $85052 \mathrm{D}$ & & \\
\hline 7 & $\begin{array}{l}\text { National } \\
\text { Instruments }\end{array}$ & $\begin{array}{l}\text { LabView } 7.1 \text { captures the data from } \\
\text { measurement instruments and plot graphs in } \\
\text { PC }\end{array}$ & LabView 7.1 & & $\begin{array}{c}\text { Software: Control } \\
\text { Equipment }\end{array}$ \\
\hline 8 & SONY VAIO & Windows XP Pro Service Pack 2 & S260P & & Run LabView 7.1 \\
\hline
\end{tabular}


DFB Laser Relative Intensity Noise (RIN) Measurement:

\begin{tabular}{|c|c|c|c|c|c|}
\hline$\#$ & Brand & Description & Model \# & Serial \# & INFO \\
\hline 1 & Kette & LD Driver \& Temperature Controller & KNN-2000LT & & \\
\hline 2 & Seastar Optics Inc. & Distributed Feedback Lasers (DFB) & PM-5501A-1550 & SP4997 & $\begin{array}{c}\text { Laser: } \\
\text { ML974A2F }\end{array}$ \\
\hline 3 & New Focus & $\begin{array}{l}\text { +/- } 15 \text { Volt Current-Limited Power } \\
\text { Supply }\end{array}$ & 901 & & 0503-10382 \\
\hline 4 & New Focus & Broadband Amplifier & 1422 & & \\
\hline 5 & Newport & Photo Detector & $\mathrm{D}-15 \mathrm{FC}$ & 1472 & \\
\hline 6 & FIS & $\begin{array}{c}\text { Optical Fiber OFNR RISER CABLE } \\
\text { 083810FT 5083 E207090(UL) } \\
\text { C(UL), SMF28E, 9/125 }\end{array}$ & SMF28E & N/A & $\begin{array}{l}\text { Single Mode, } \\
\text { FC Connector }\end{array}$ \\
\hline 7 & HP & Spectrum Analyzer & $8593 \mathrm{~A}$ & $3034 \mathrm{~A} 00883$ & \\
\hline 8 & Fluke & Digital Multi Meter & 87 & EE5710 & \\
\hline
\end{tabular}

Fabry-Pérot Laser RIN Measurement:

\begin{tabular}{|c|c|c|c|c|c|}
\hline$\#$ & Brand & Description & Model \# & Serial \# & INFO \\
\hline 1 & Power One & FP Laser Power Supply & HCBB-75W-A & N/A & \\
\hline 2 & HP & Fabry-Pérot Lasers & $\begin{array}{c}\text { \#1 \& \#2 HP83400A x } 2 \\
\text { \#3 HP83401A } \\
\text { \#4 HP83402A }\end{array}$ & $\begin{array}{l}\text { 2850A00108 } \\
\text { 2860A00114 } \\
\text { 2860A00108 } \\
\text { 2826A00203 }\end{array}$ & $\begin{array}{c}\text { \#1 \& \#2 SMF } \\
\text { \#3 MMF } \\
\text { \#4 Unknown }\end{array}$ \\
\hline 3 & New Focus & $\begin{array}{l}+/-15 \text { Volt Current-Limited Power } \\
\text { Supply }\end{array}$ & 901 & & 0503-10382 \\
\hline 4 & New Focus & Broadband Amplifier & 1422 & & \\
\hline 5 & Picosecond Pulse Lab & 12.5 Gpbs Driver Amplifier & 5865 & 0613921 & $12 \mathrm{GHz}$ BW \\
\hline 6 & $\begin{array}{l}\text { Topward Electric } \\
\text { Instrument }\end{array}$ & Dual Tracking DC Power Supply & TPS-4000 & EE 4949 & $\begin{array}{c}\text { Short the } \\
\text { middle }+\&-\end{array}$ \\
\hline 7 & Newport & Photo Detector & D-15FC & 1472 & \\
\hline 8 & FIS & $\begin{array}{c}\text { Optical Fiber OFNR RISER CABLE } \\
\text { 083810FT 5083 E207090(UL) } \\
\text { C(UL), SMF28E, 9/125 }\end{array}$ & SMF28E & N/A & $\begin{array}{l}\text { Single Mode, } \\
\text { FC Connector }\end{array}$ \\
\hline
\end{tabular}




\begin{tabular}{|c|c|c|c|c|}
\hline 9 & HP & Spectrum Analyzer & $8593 \mathrm{~A}$ & 3034A00883 \\
\hline 10 & Fluke & Digital Multi Meter & 87 & EE5710 \\
\hline
\end{tabular}

\section{VCSEL Optical Pumping Experiment:}

\begin{tabular}{|c|c|c|c|c|c|c|}
\hline$\#$ & Brand & Description & Model \# & Serial \# & INFO & Quantities \\
\hline 1 & Anritsu & SMA Cable & $3670 \mathrm{~K} 50-2$ & & SC3855 & 2 \\
\hline 2 & FIS & $\begin{array}{c}\text { Optical Fiber OFNR RISER CABLE 083810FT } \\
5143 \text { E207090(UL) C(UL), 62.5/125 }\end{array}$ & S27ULU2fis & N/A & $\begin{array}{l}\text { Multi-Mode, } \\
\text { LC/FC } \\
\text { Connector }\end{array}$ & 1 \\
\hline 3 & Honeywell & VCSEL, $4.25 \mathrm{Gbps} 850 \mathrm{~nm}$ & HFE4192-901 & & $\begin{array}{c}\text { Laser: } \\
\text { VCSEL (LC } \\
\text { TOSA) }\end{array}$ & 2 \\
\hline 4 & HP & Network Analyzer & 8720B & EE5165 & $\begin{array}{c}130 \mathrm{MHz}-20 \\
\mathrm{GHz}\end{array}$ & 1 \\
\hline 5 & HP & Calibration Kit 2.5mm Economy Calibration Kit & $85052 \mathrm{D}$ & & & 1 \\
\hline 6 & $\begin{array}{c}\text { ILX } \\
\text { Lightwave }\end{array}$ & Current Control & LDX-3207 & & $\begin{array}{l}\text { Obsolete, to } \\
\text { drive TEST } \\
\text { Laser }\end{array}$ & 1 \\
\hline 7 & $\begin{array}{c}\text { ILX } \\
\text { Lightwave }\end{array}$ & Laser Diode Current and Temperature Controller & LDC-3744B & 37443707 & $\begin{array}{c}\text { To drive } \\
\text { Master Laser }\end{array}$ & 1 \\
\hline 8 & $\begin{array}{c}\text { ILX } \\
\text { Lightwave }\end{array}$ & Temperature Controlled Laser Diode Mount & LDM-4407 & $\begin{array}{l}44071645 \\
\text { DL983391 }\end{array}$ & $\begin{array}{c}\text { Manufacture } \\
\text { Date: } \\
1 / 13 / 2005\end{array}$ & 1 \\
\hline 9 & $\begin{array}{c}\text { National } \\
\text { Instruments }\end{array}$ & GPIB to Hi-Speed USB converter & $\begin{array}{c}\text { GPIB-USB- } \\
\text { HS }\end{array}$ & 11B4F84 & & 1 \\
\hline 10 & $\begin{array}{c}\text { National } \\
\text { Instruments }\end{array}$ & $\begin{array}{c}\text { LabView } 7.0 \text { captures the data from measurement } \\
\text { instruments and plot graphs in PC }\end{array}$ & LabView 7.0 & & $\begin{array}{c}\text { Software: } \\
\text { Control } \\
\text { Equipment }\end{array}$ & 1 \\
\hline 11 & New Focus & +/- 15 Volt Current-Limited Power Supply & 901 & & 0503-10382 & 1 \\
\hline 12 & New Focus & Broadband Amplifier & 1422 & & & 1 \\
\hline 13 & Newport & Photo Detector & $\mathrm{D}-15 \mathrm{FC}$ & 1472 & & 1 \\
\hline 14 & $\begin{array}{l}\text { Phoenix } \\
\text { Photonics }\end{array}$ & Coupler $50 \%$-FC,50\%-LC, $100 \%$ LC, $3 \mathrm{~mm}$ Cable & \begin{tabular}{|c|} 
MMC-1-850- \\
5050-2-B-X
\end{tabular} & 60413142 & $\begin{array}{l}\text { Multi-Mode } \\
\text { Coupler }\end{array}$ & 1 \\
\hline
\end{tabular}




\begin{tabular}{|c|c|c|c|c|c|c|}
\hline 15 & $\begin{array}{c}\text { Picosecond } \\
\text { Pulse Labs }\end{array}$ & Bias Tee & 5541A & $5968 / 97$ & 26Ghz, 8ps & 1 \\
\hline 16 & SONY VAIO & Windows XP Pro Service Pack 2 & S260P & & $\begin{array}{c}\text { Run LabView } \\
7.0\end{array}$ & 1 \\
\hline 17 & & SMA Connector 2-sided & & & & 1 \\
\hline 18 & & SMA to BNC connector & & & & 1 \\
\hline
\end{tabular}




\section{APPENDIX B: Algorithm for Indexing and Reading from the}

\section{Indexed Optical Power Spectrum Database}

\section{Optical Spectrum Analyzer Automatic Data Recording at Different Input Current at Various Temperatures.}

A LabView code is written to control the HP Optical Spectrum Analyzer to automatically record the optical spectra at different input current of the laser diode at various temperatures. The drive current ranges from $0 \mathrm{~mA}$ to $19.5 \mathrm{~mA}$ with increments from 0.5 $\mathrm{mA}$ to $1 \mathrm{~mA}$. The temperature ranges from $-7{ }^{\circ} \mathrm{C}, 3{ }^{\circ} \mathrm{C}, 13{ }^{\circ} \mathrm{C}$, to $83{ }^{\circ} \mathrm{C}$ with increments of 10 degrees Celsius. The recorded optical spectrum data are stored in a text file called totalABXY.txt which is read by another LabView code to generate optical spectrum plots at a constant temperature with various input currents or a constant input current at various temperatures. The totalABXY file contains four columns from left to right. The first column is the temperature in Celsius, the second column is input current in $\mathrm{mA}$, the third and forth columns are the optical spectrum with optical wavelength in nano meter and optical power in $\mathrm{dBm}$ respectively.

The input currents increment from top to bottom for each optical spectrum while the temperature increment only after all the input currents have been incremented. If multiple optical spectra are needed to plot beside each other at either constant temperature and various input currents or constant input current at various temperatures, then an algorithm has to be used to read and output the optical spectra.

Since searching for the optical spectra at particular constant temperatures or input currents is time consuming; therefore, the data has to be indexed to speed up the searching process.

\section{The Indexing Algorithm (Indexing v1.0.vi)}

Inputs: Temperature Array Size = A_Size, Current Array Size = B_Size, Optical Spectrum Array Size $=$ XYSize

Outputs: StartA, StopA, StartB, StopB. Data Output Directory in Excel spreadsheet

format.

LabView Algorithm

0: Start to do indexing for plotting data from totalABXY String Array

A_RangeSize $=$ B_Size $*$ XYSize;

B_RangeSize $=$ A_Size $*$ XYSize;

\section{1: Initializes the arrays}


Start A = zeros(A Temperature Size);

Stop A = zeros(A Temperature Size);

Start B = zeros(B Input Current Size);

Stop B = zeros(B Input Current Size);

B_ArrayCounter $=0$; 
2: Generates the Start or Stop Index values array for $A$ and $B$ arrays

for $(\mathrm{i}=0 ; \mathrm{i}<$ A_Size; $\mathrm{i}++)$

\{

StartA[i] = i*A_RangeSize;

StopA $[\mathrm{i}]=(\mathrm{i}+1)^{*}$ A_RangeSize-1;

for $(j=0 ; j<$ B_Temperature_Size $; j++)$

\{

StartB $[\mathrm{j}]=$ B_ArrayCounter*XYSize;

StopB $[\mathrm{j}]=\left(\mathrm{B} \_\right.$ArrayCounter+1 $) * X Y$ Size- 1 ;

\}

\}

3: Save the $A$ and $B$ arrays Indices into four text files to be accessed by the indices reader for plotting.

Save the following data into text files:

StartA.txt $=$ save as text $($ StartA $)$;

StopA.txt $=$ save as text $($ StopA $)$;

StartB.txt $=$ save as text $($ StartB $)$;

StopB.txt = save as text $($ StopB $)$; 


\section{Reading Indexed Algorithm (Reading Indexed Data v1.0.vi)}

Inputs are in text file, .TXT format:

- A constant temperature $\left({ }^{\circ} \mathrm{C}\right)$ at varying input current $(\mathrm{mA})$

- B constant input current $(\mathrm{mA})$ at varying constant temperature $\left({ }^{\circ} \mathrm{C}\right)$

- Total ABXY Input Directory, Read A (Temperature), Read B (Input Current), Read X (Wavelength/Frequency), Start A (Temperature Indexes), Stop A (Temperature Indexes), Start B (Input Current Indexes), Stop B (Input Current Indexes)

- Linear/Log scale

- Wavelength/Frequency

Outputs are in Excel Spreadsheet, .XLS format:

- Output Final Constant A varying B

- Output Final Constant B Varying A

\section{LabView Algorithm}

0: Read in the TotalABXY.txt arrays with the indexed arrays for A (Input Current, mA), B (Temperature, ${ }^{\circ} \mathrm{C}$ ), $\mathrm{X}$ (Wavelength, $\mathrm{nm}$ ), and Y (Power, $\mathrm{dBm}$ )

A_Constant (the Constant Temperature, ${ }^{\circ} \mathrm{C}$, by varying the input current, $\mathrm{mA}$ ) $=24^{\circ} \mathrm{C}$ by default;

B_Constant (the constant input current, $\mathrm{mA}$, by varying the temperature, ${ }^{\circ} \mathrm{C}$ ) $=15 \mathrm{~mA}$ by default;

readA = Read arrayA.txt (Temperatures);

readB $=$ Read arrayB.txt (Input Currents);

readX = Read arrayX.txt (Wavelength/Frequency);

readStartA = Read StartA.txt (Start Temperature Indexes);

readStopA = Read StopA.txt (Stop Temperature Indexes);

readStartB = Read StartB.txt (Start Input Current Indexes);

readStopB = Read StopB.txt (Stop Input Current Indexes);

readTotalABXY = Read totalABXY.txt;

1: Get Array Sizes

A_Size $=\operatorname{get} \operatorname{Size}(\operatorname{Read} \mathrm{A})$;

B_Size $=$ getSize $(\operatorname{ReadB})$;

XY_Size = getSize $(\operatorname{ReadX})$;

Total_ABXY_Size $=$ getSize $($ ReadTOTALABXYString $)$;

2a: Searching the Index of the Total ABXY String for Constant A, varying with B given with a specific $A$ input.

2a.0:

StartAIndex = search A_Constant within readStartA;

StopAIndex = search A_Constant within readStopA; 
2a.1: Temp_Constant_Temperature_Total_String = StopAIndex -StartAIndex +1 ; 2a.2: Initializes the $X$ versus $Y$ array, Multiple $Y$ Arrays at constant Temperature $A$ varying B $=$ Multi_Y

Multi_Y=emptyString[XYSize+1][B_Size];

2a.3: Initializes the constant $B$ and constant $Y$ counters

//Constant Temperature Y Array Counter;

count_Y $=0$;

//Constant Temperature B Array Counter;

count_B =0;

2b.0: Searching the Index of the Total ABXY String for constant B, varying with A given with a specific $B$ input.

StartBIndexArray = search B_Constant within readStartB;

StopBIndexArray = search B_Constant within readStopB;

B_IndexCounter $=0$;

\section{2b.1:}

for $(\mathrm{i}=0 ; \mathrm{i}<$ StopBIndexArray; $\mathrm{i}++)$

\{

B_IndexCounter $=$ B_IndexCounter $+1+$ StopBIndexArray[i] -

StartBIndexArray[i];

\}

2b.2: Initializes with empty strings for the Temporary Constant Input Current Total String

Temp_Constant_Input_Current_Total_String = emptyString[B_IndexCounter];

2b.3: Resets the B_IndexCounter to 0;

2.3: Start to read data from the Total String for Constant A (Temperature) by varying B (Current)

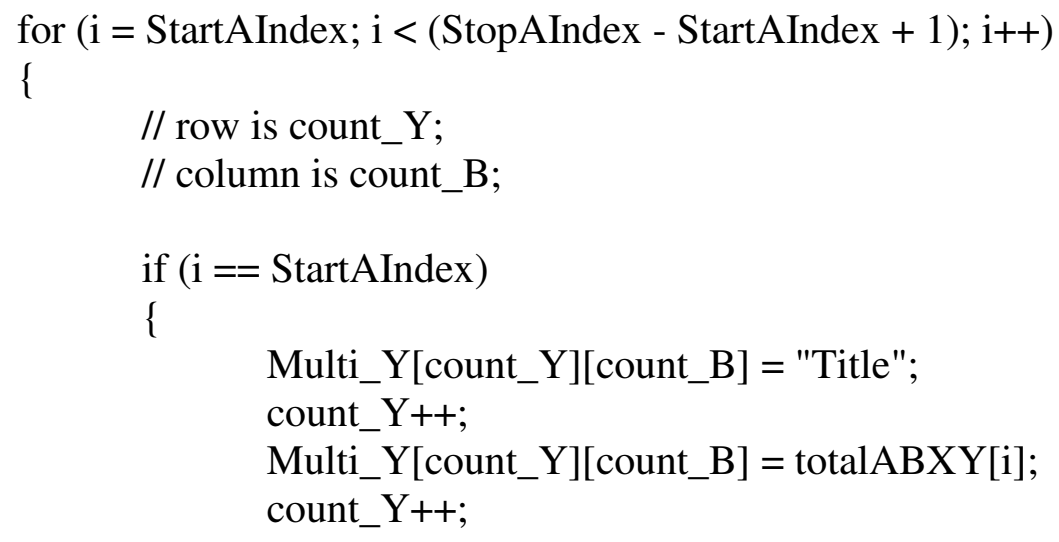




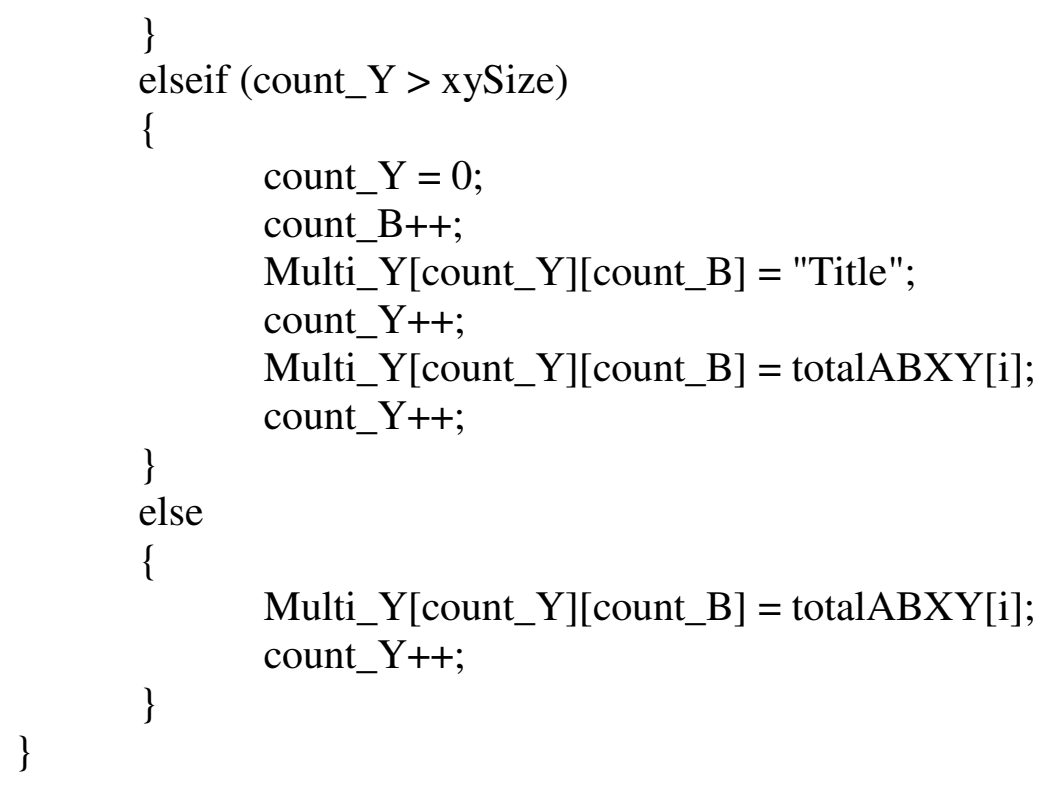

4: Save the Multi_Y array into output final constant A varying B;

5: Initialize the values and arrays before generating constant $B$ with varying $A, X-Y$ plot; count_A $=0$;

6: Generates optical spectrum arrays with Constant Input Current with varying Temperature for $(i=0 ; i<=$ StopBIndexArray.length; $i++)$

\{

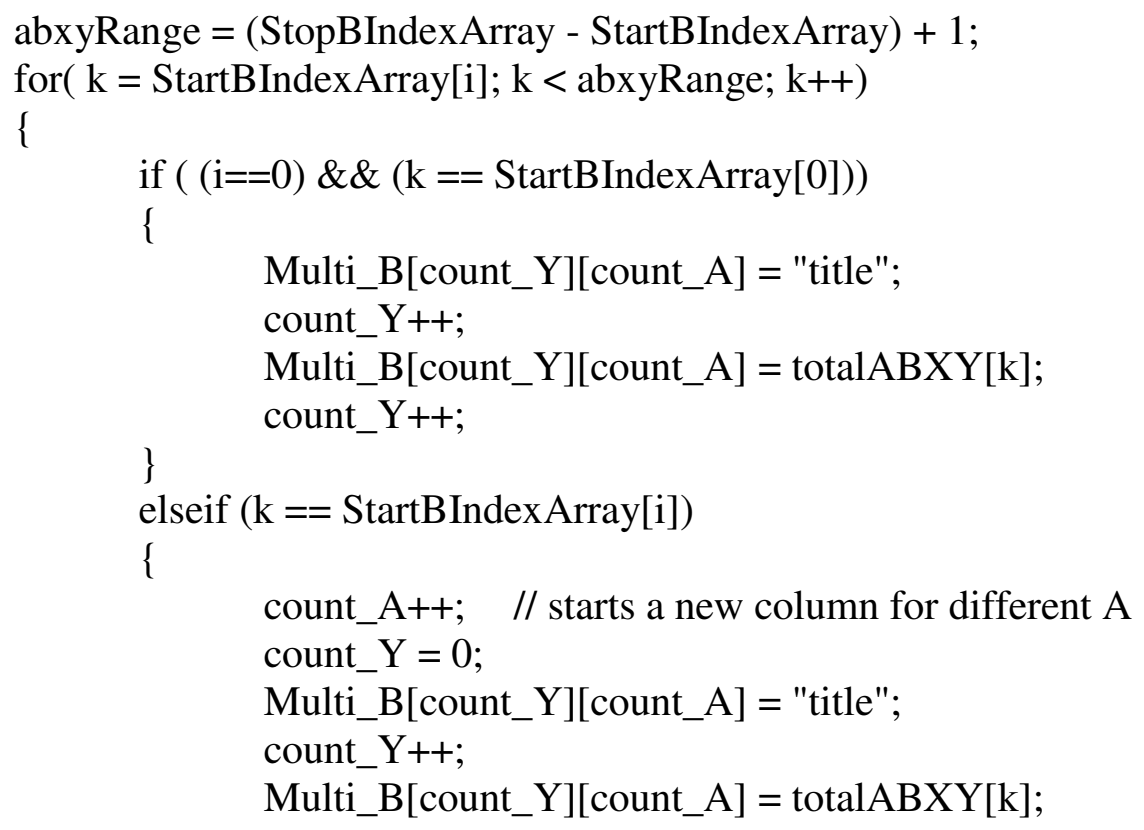




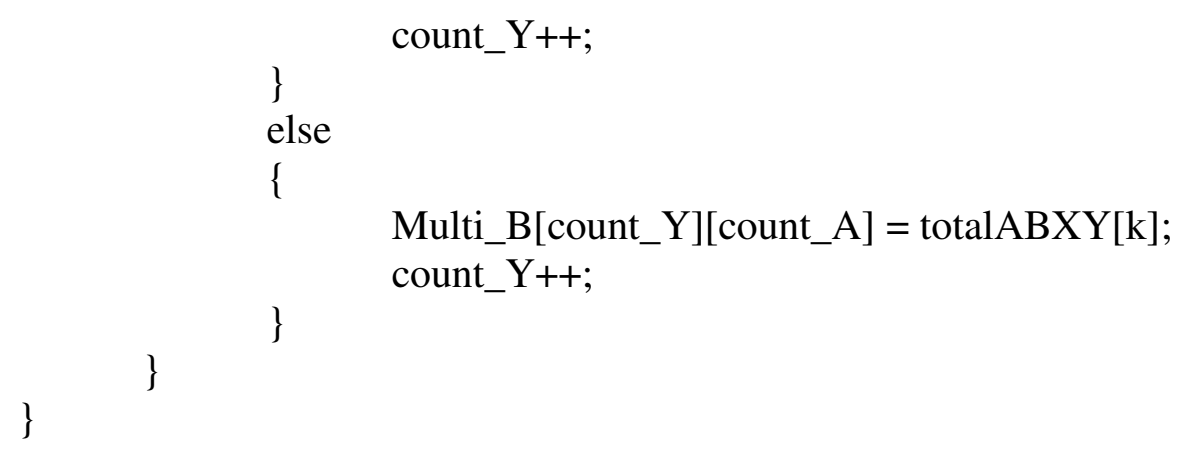

7: Outputs the Final Constant B varying A array 


\section{Indexing v1.0.vi LabView Source Codes}

\section{Indexing Front Panel}

\section{INDEXING the TOTAL ABXY ARRAY}

This Algorithm performs indexing to speed up the TOTAL ABXY Array search process

It takes in one address directory string, A, B, and X-Y Size as input parameters.

It then outputs the following arrays: StartA, StopA, StartB, StopB

Simulation Parameters

A - Input Temperature $(\mathrm{oC})$

Output Directory String Address

B - Input Current ( $\mathrm{m}$

$X$ - Wavelength $(\mathrm{nm})$

C:ITemplTesting

$\mathrm{X}=200 \mathrm{Y}$ - Power $(\mathrm{W}$ or $\mathrm{dBm})$

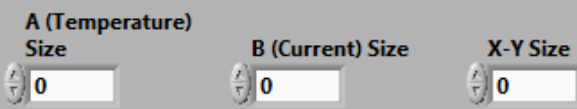

The Start and Stop Aarray contains the INDEX of TOTAL ABXY String Array for Each Input Temperature

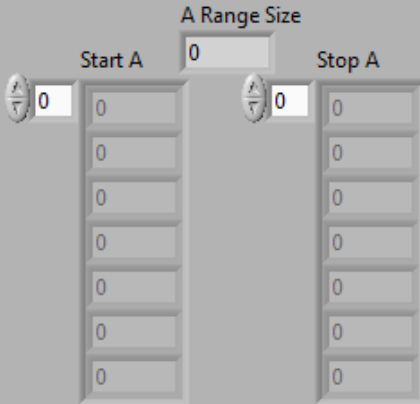

The Start and Stop Aarray contains the index of TOTAL ABXY String Array Each Input Current and Temperature - Rows are Input Current Index

B Range Size $\quad$ - Columfarte Pnput Temperature Index

0

B Array Counter

5. 0

능 0

0

\begin{tabular}{|l|l|l|l|l|}
\hline 0 & 0 & 0 & 0 & 0 \\
\hline 0 & 0 & 0 & 0 & 0 \\
\hline 0 & 0 & 0 & 0 & 0 \\
\hline 0 & 0 & 0 & 0 & 0 \\
\hline 0 & 0 & 0 & 0 & 0 \\
\hline 0 & 0 & 0 & 0 & 0 \\
\hline 0 & 0 & 0 & 0 & 0 \\
\hline
\end{tabular}

\begin{tabular}{|c|c|c|c|c|c|}
\hline \multicolumn{6}{|c|}{ Stop B } \\
\hline 0 & 0 & 0 & 0 & 0 & 0 \\
\hline \multirow[t]{6}{*}{0} & 0 & 0 & 0 & 0 & 0 \\
\hline & 0 & 0 & 0 & 0 & 0 \\
\hline & 0 & 0 & 0 & 0 & 0 \\
\hline & 0 & 0 & 0 & 0 & 0 \\
\hline & 0 & 0 & 0 & 0 & 0 \\
\hline & 0 & 0 & 0 & 0 & 0 \\
\hline
\end{tabular}




\section{Indexing.0}

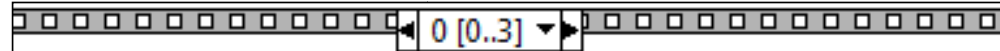

Start to do INDEXING for Ploting Data from the totalABXY String Array

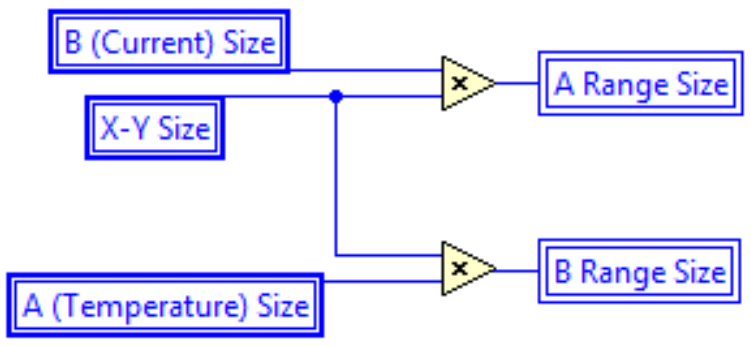

\section{Indexing.1}

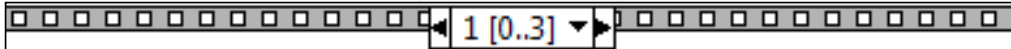

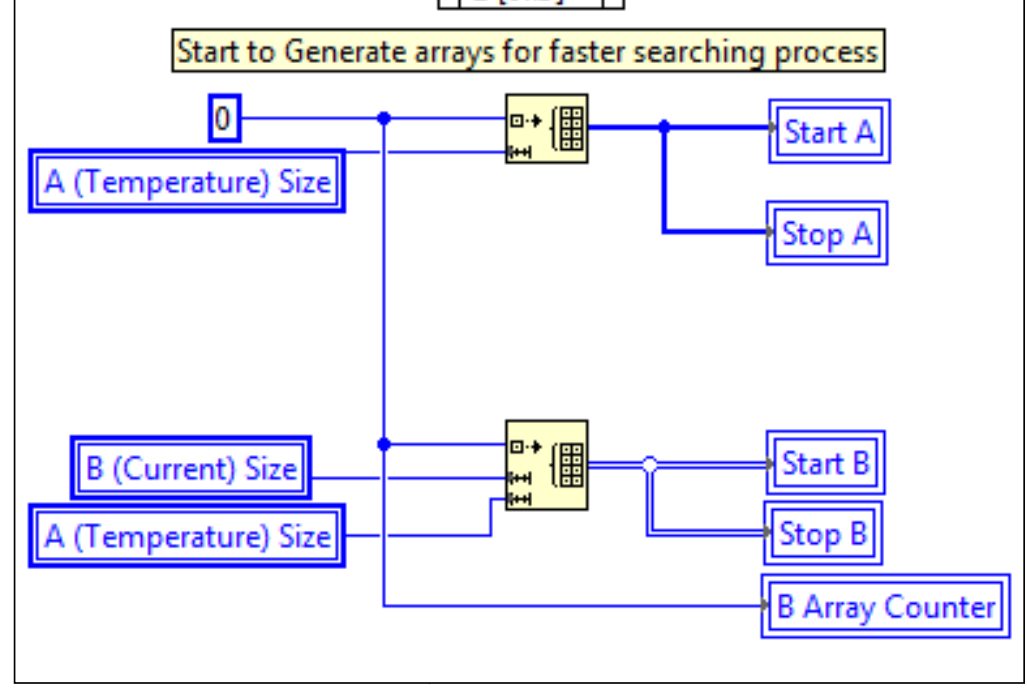




\section{Indexing.2}

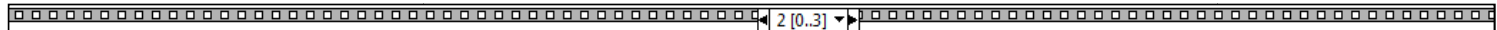

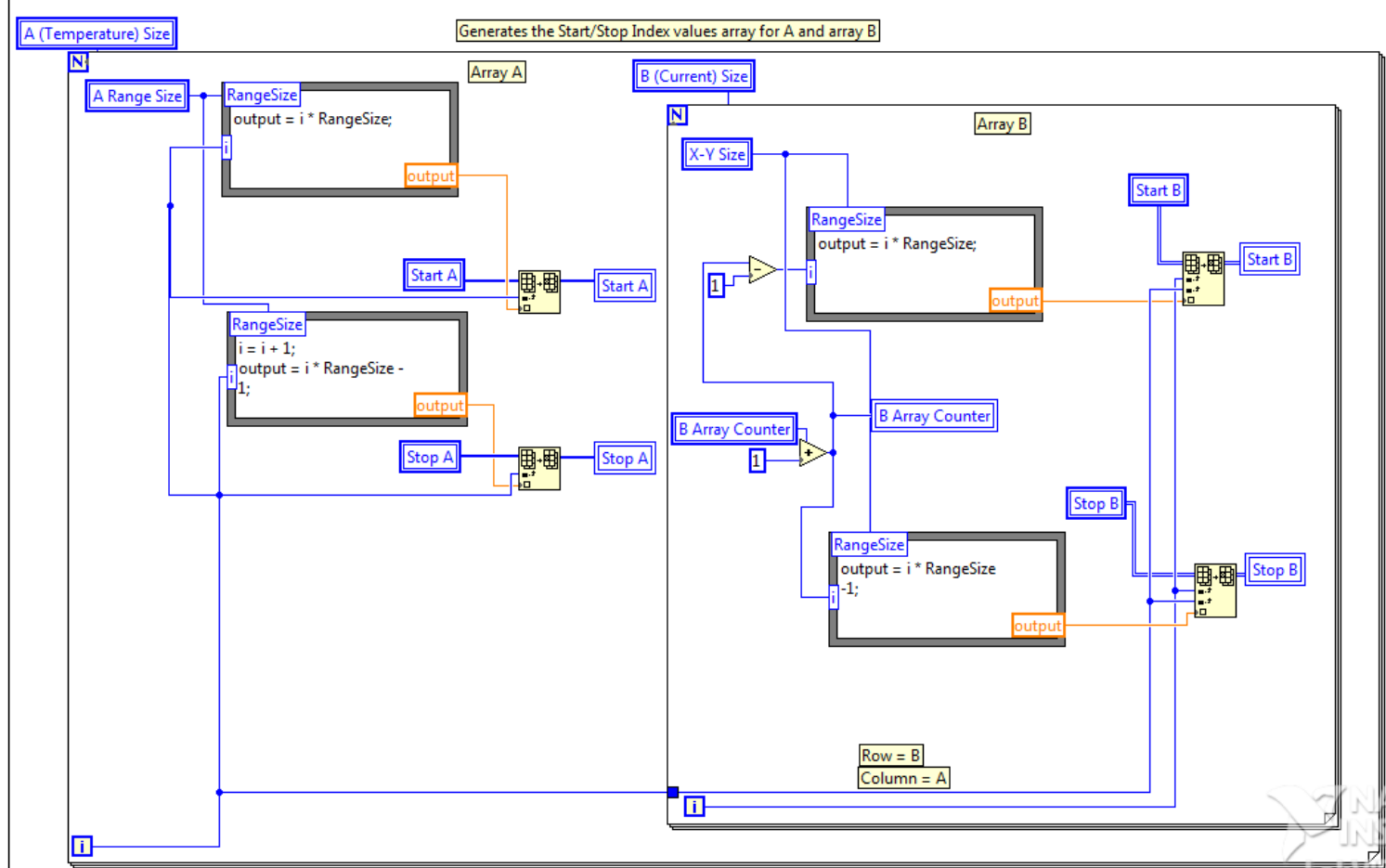

Indexing.3

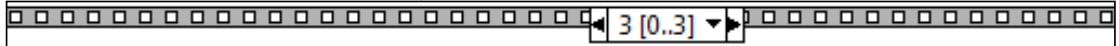
Save the A and B arrays' Index into text files to be accessed by the search algorithm for plotting Output Directory String Address

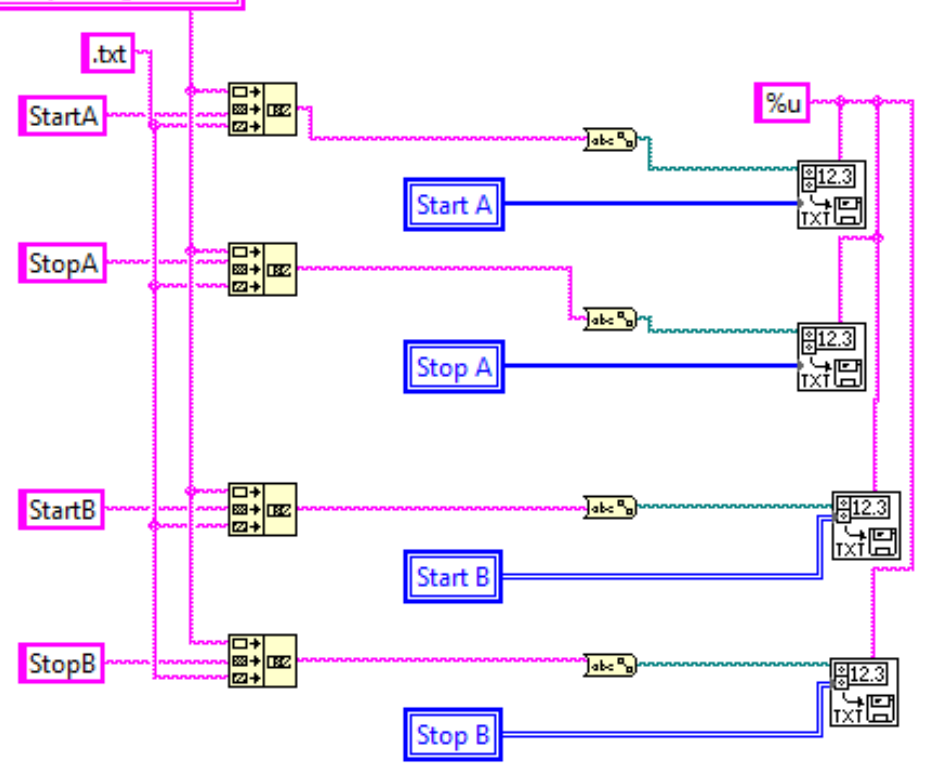




\section{Reading Indexed Data v1.0.vi LabView Source Code}

\section{Read Indexed Data Front Panel}

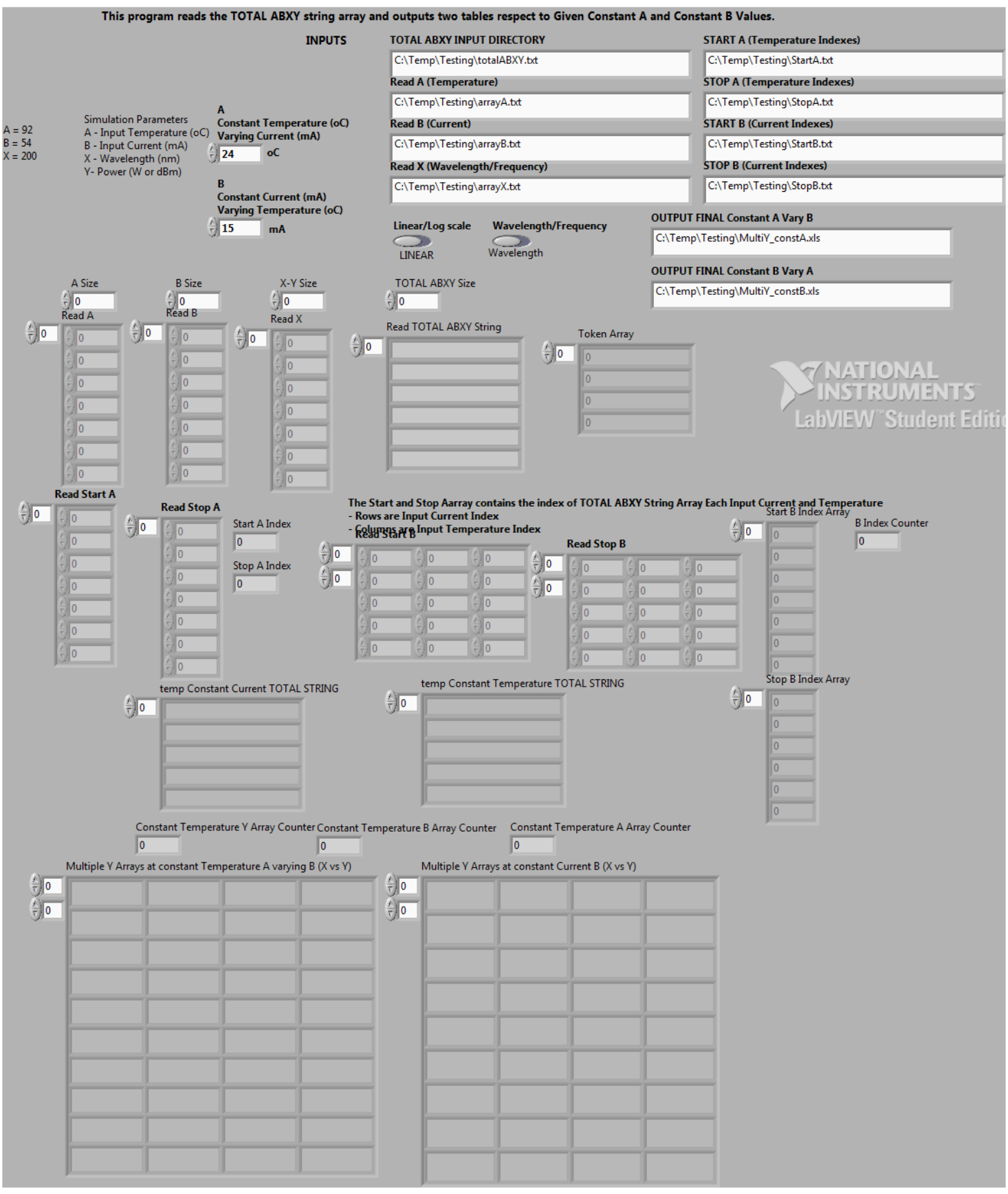




\section{Read Indexed.0}

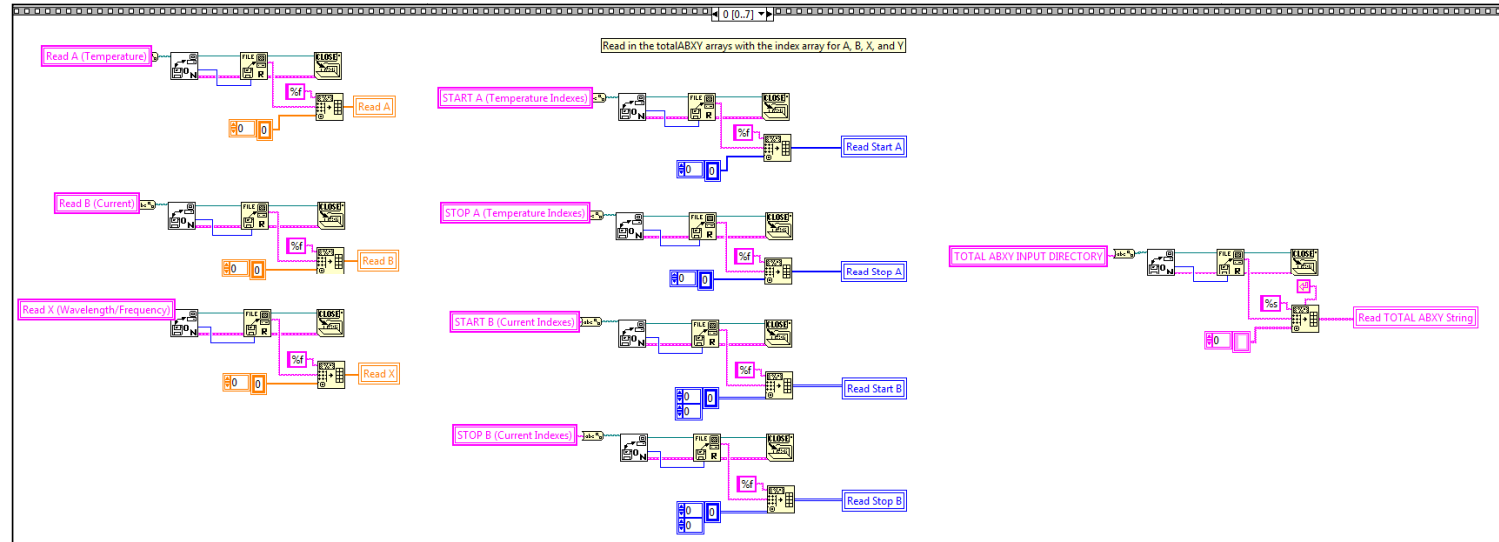

\section{Read Indexed.1}

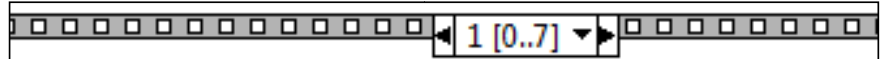

Get Array Size

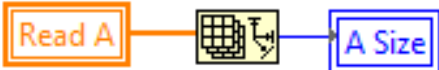

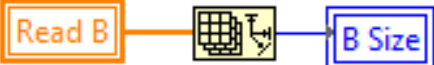

Read X 遇輱 X-Y Size

Read TOTAL ABXY String 


\section{Read Indexed.2.0}

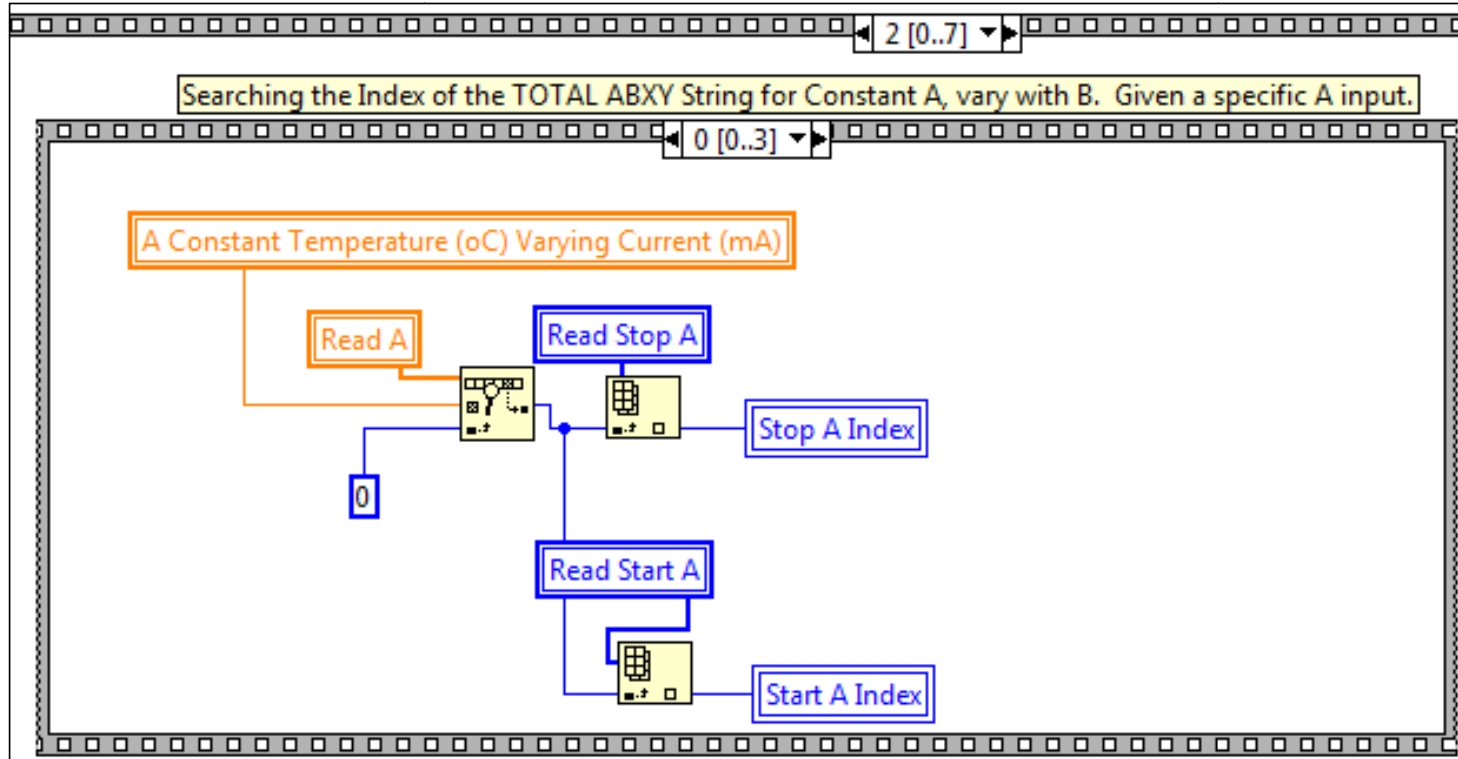

Searching the Index of the TOTAL ABXY String for Constant B, vary with A. Given a specific B input. Bowstant Current (mA) Varying Temperature (oC)

$$
0 \text { B Index Counter }
$$




\section{Indexed 2.1}

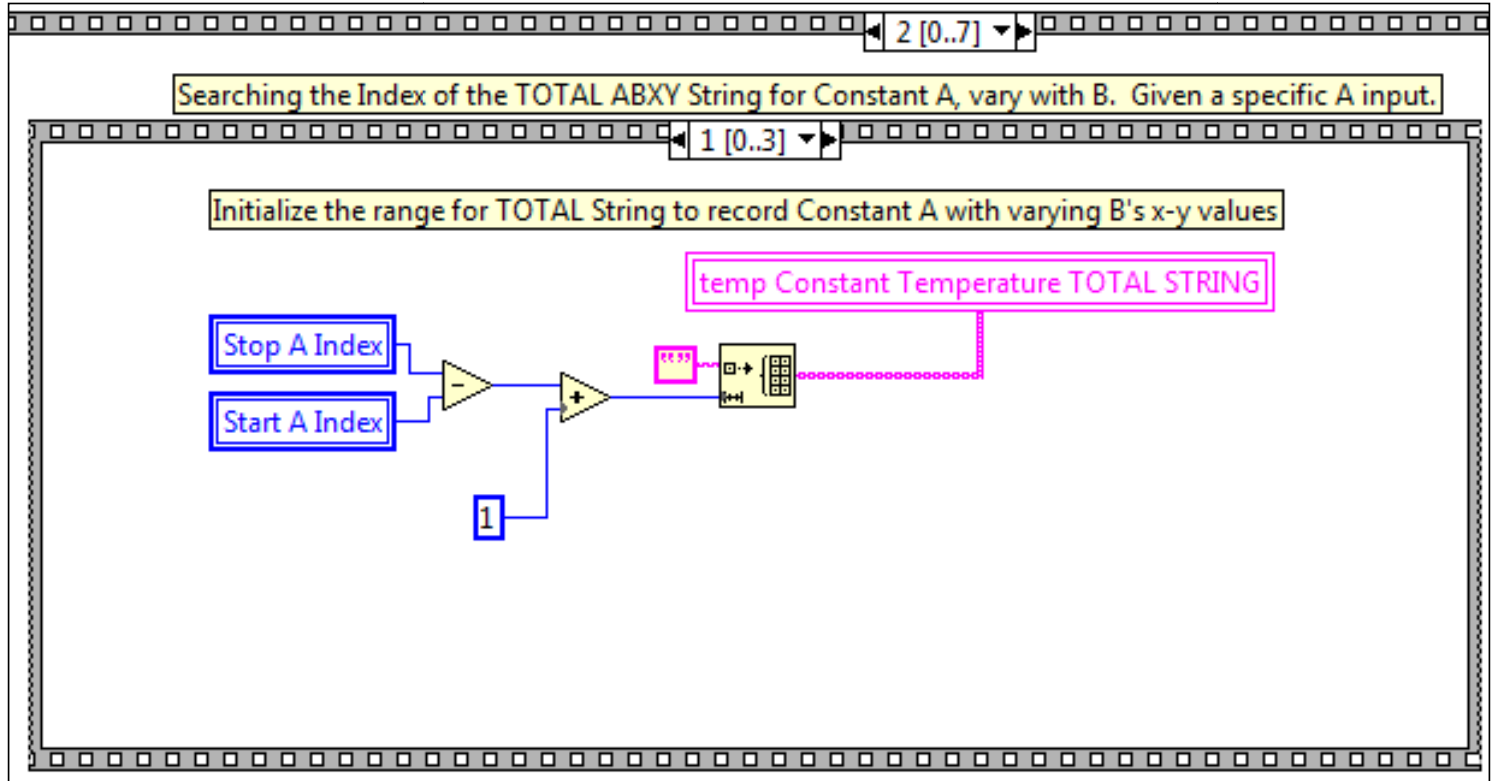

Searching the Index of the TOTAL ABXY String for Constant B, vary with A. Given a specific B input.

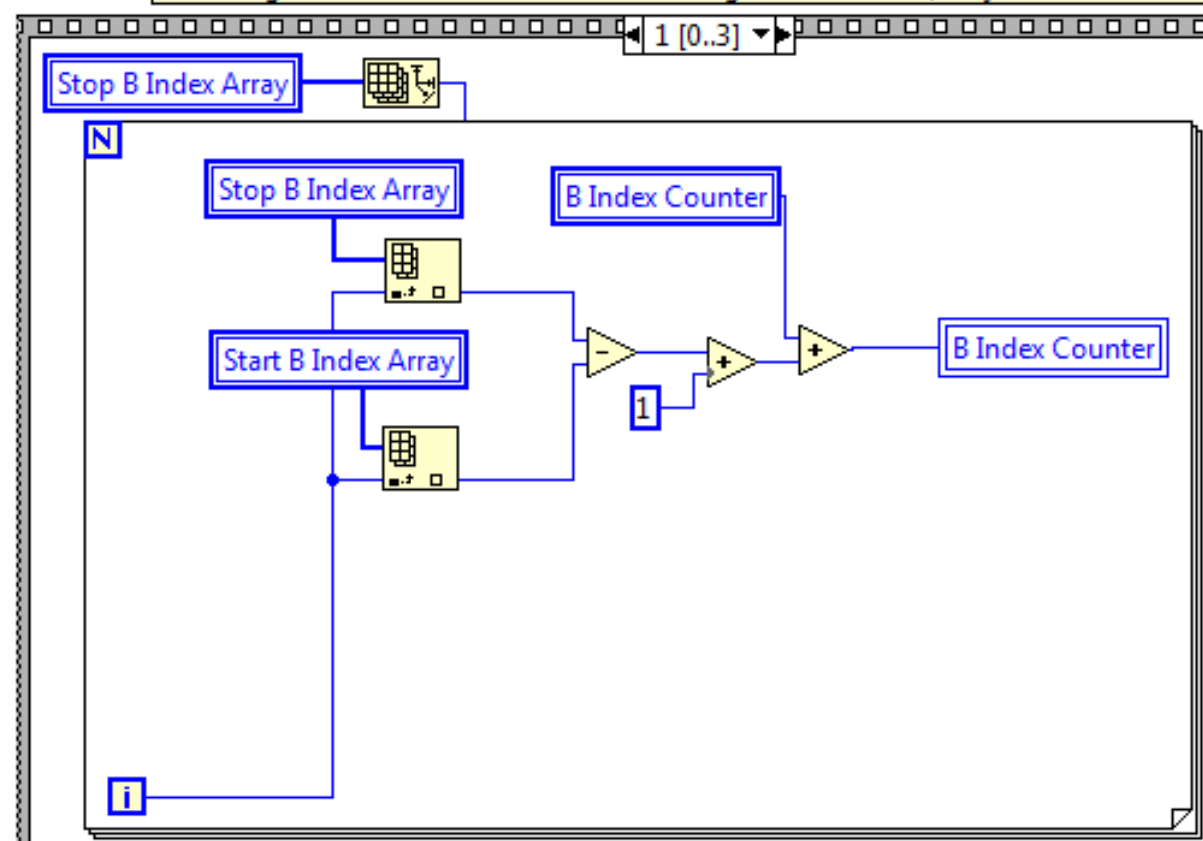




\section{Indexed 2.2}

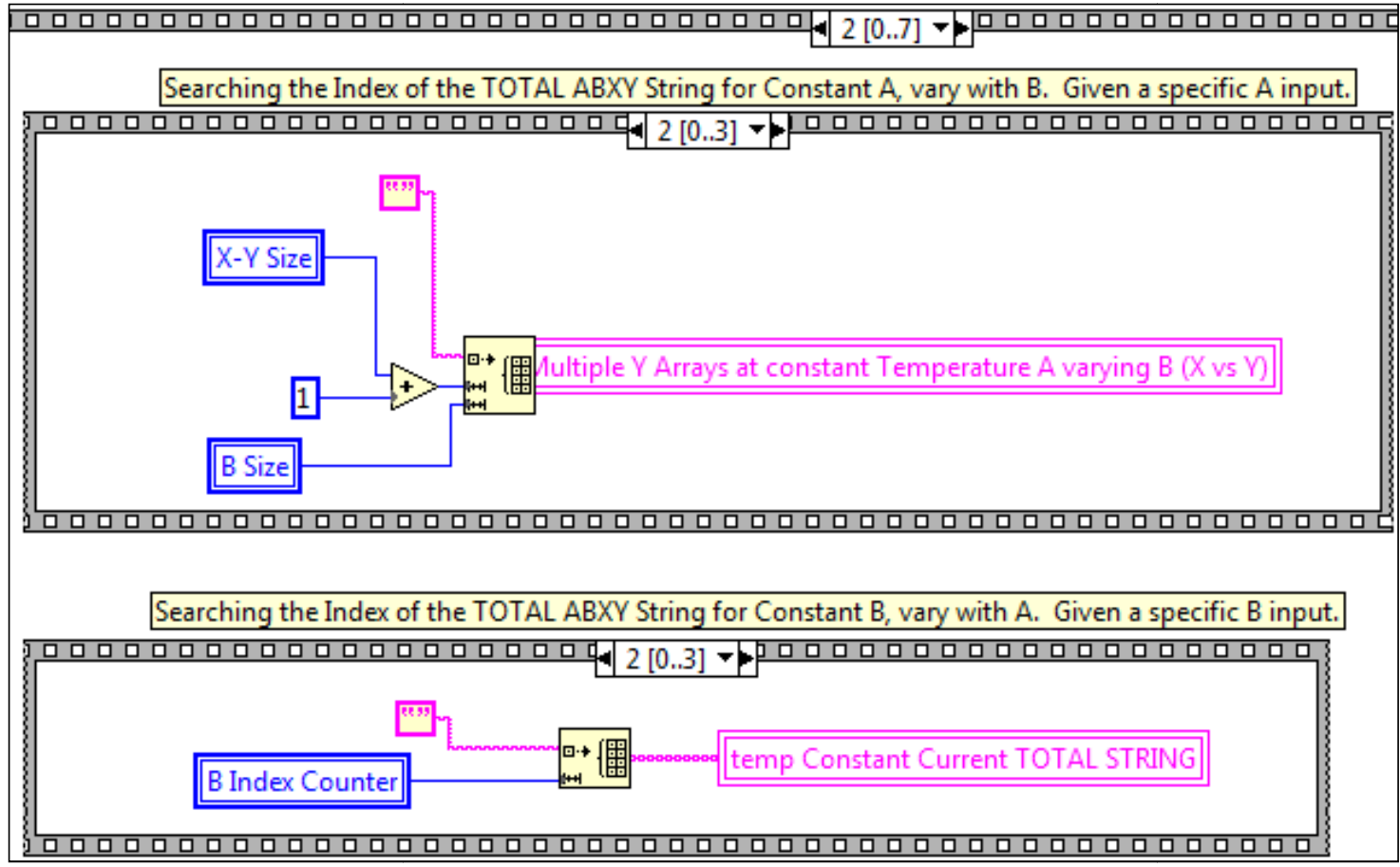

\section{Indexed 2.3}

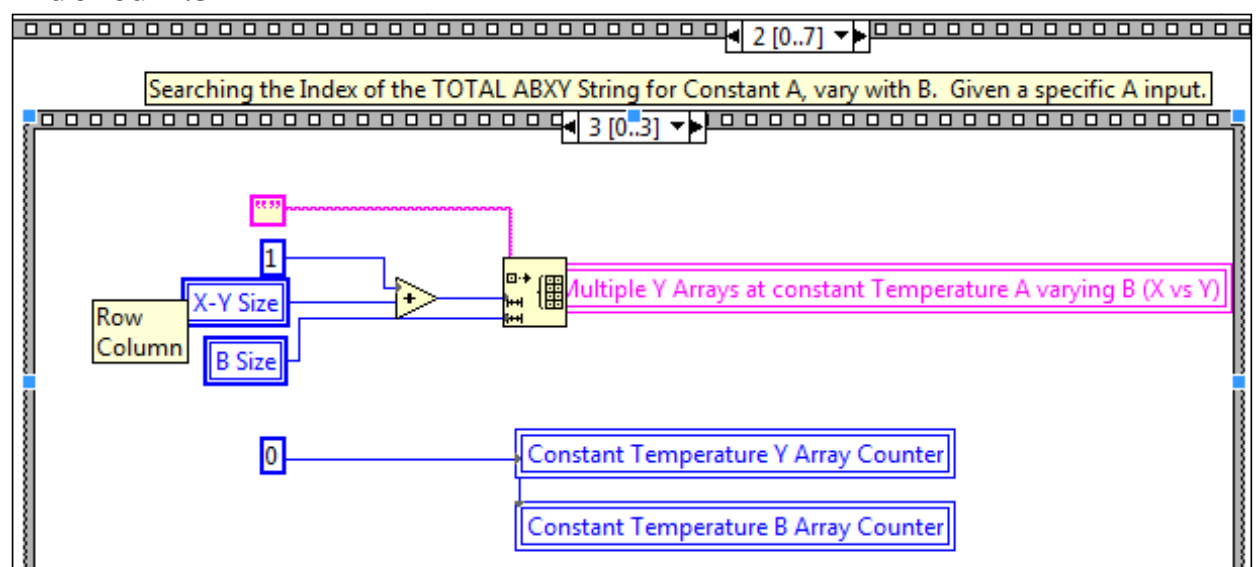

Searching the Index of the TOTAL ABXY String for Constant B, vary with A. Given a specific B input.

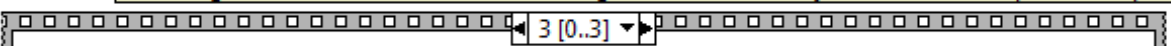

$$
0 \text { B Index Counter }
$$

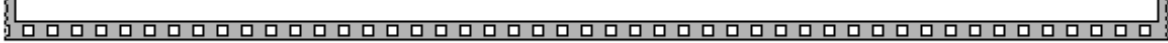

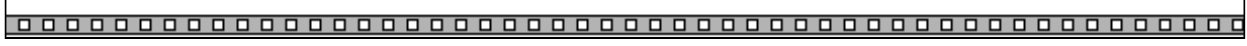




\section{Read Indexed.3.FALSE.FALSE.0}

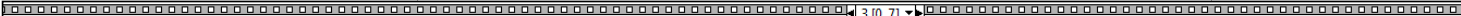

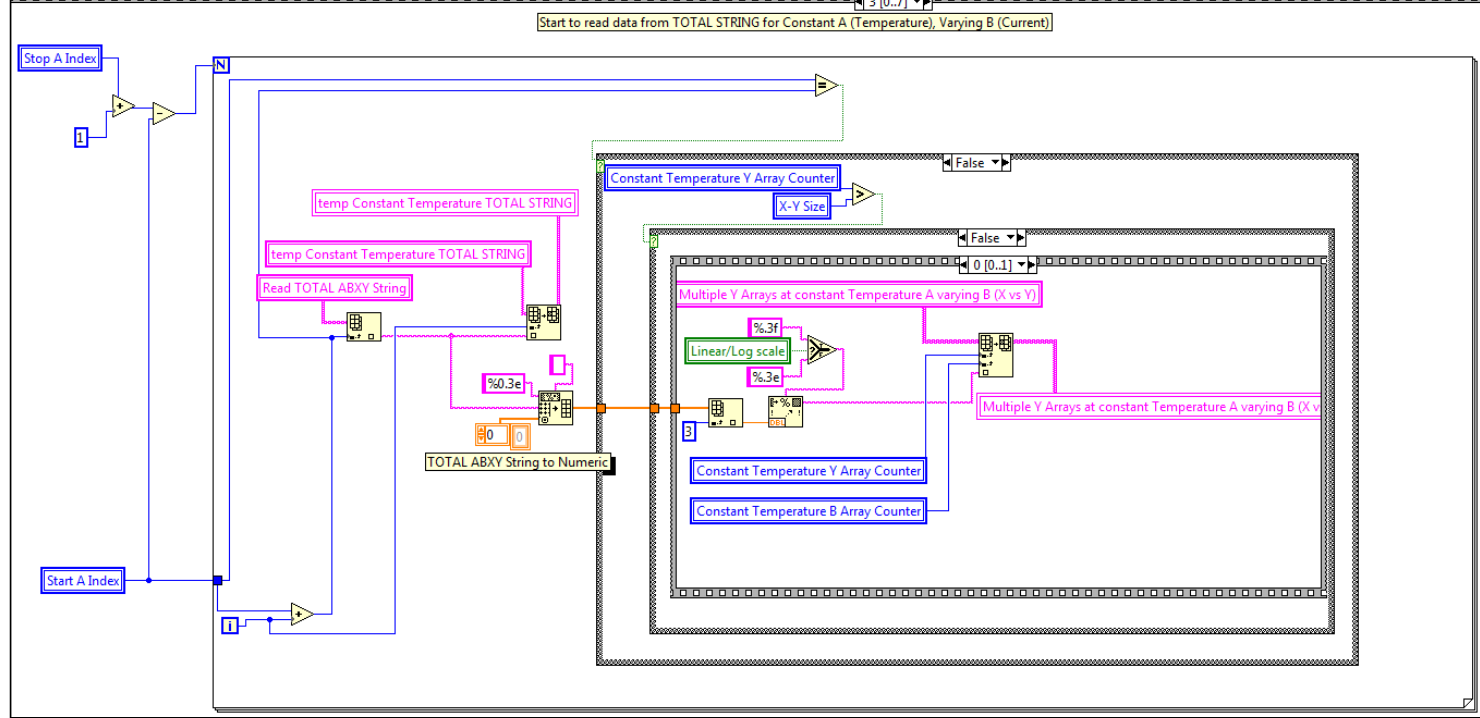

\section{Read indexed.3.FALSE.FALSE.1}

$000000000000000000000000000000000000000000000000000000000000000043[0.7]+8000000000000000000000000000000000000000000$

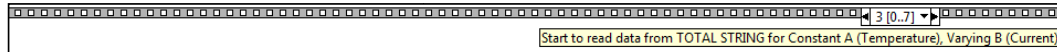

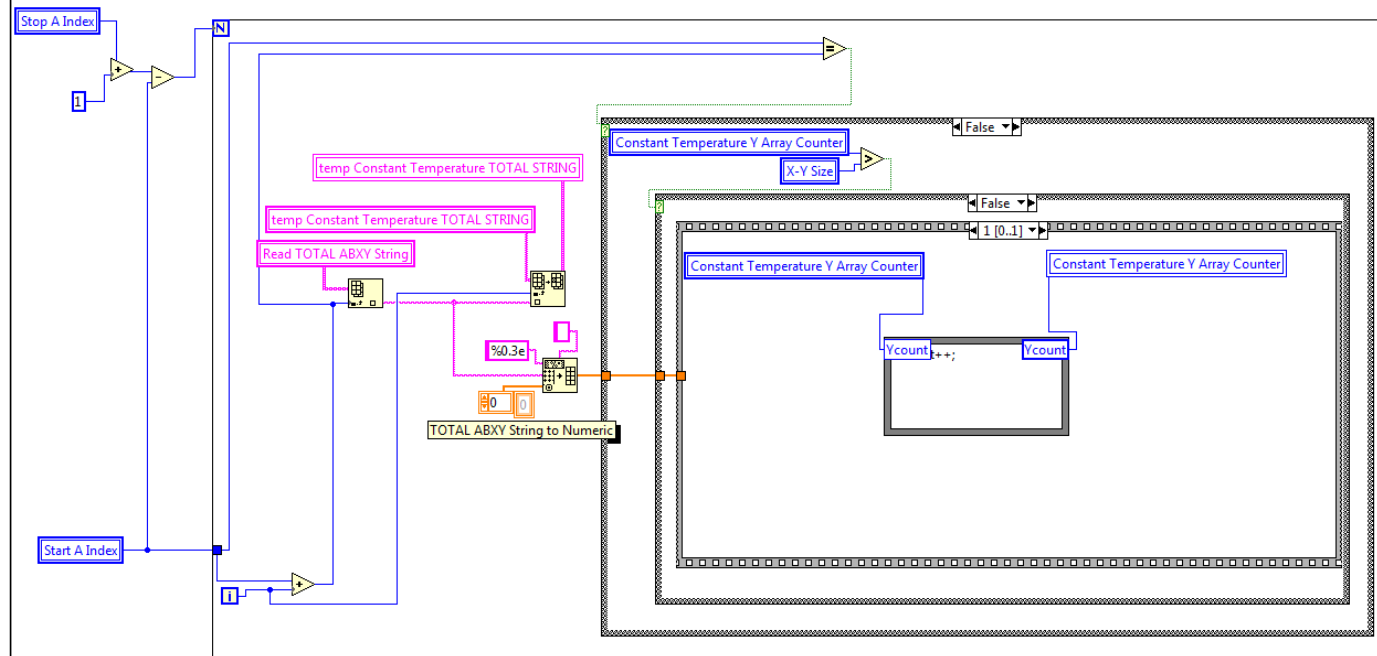




\section{Read indexed.3.FALSE.TRUE.0}

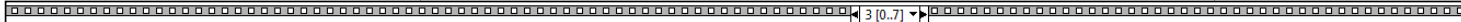

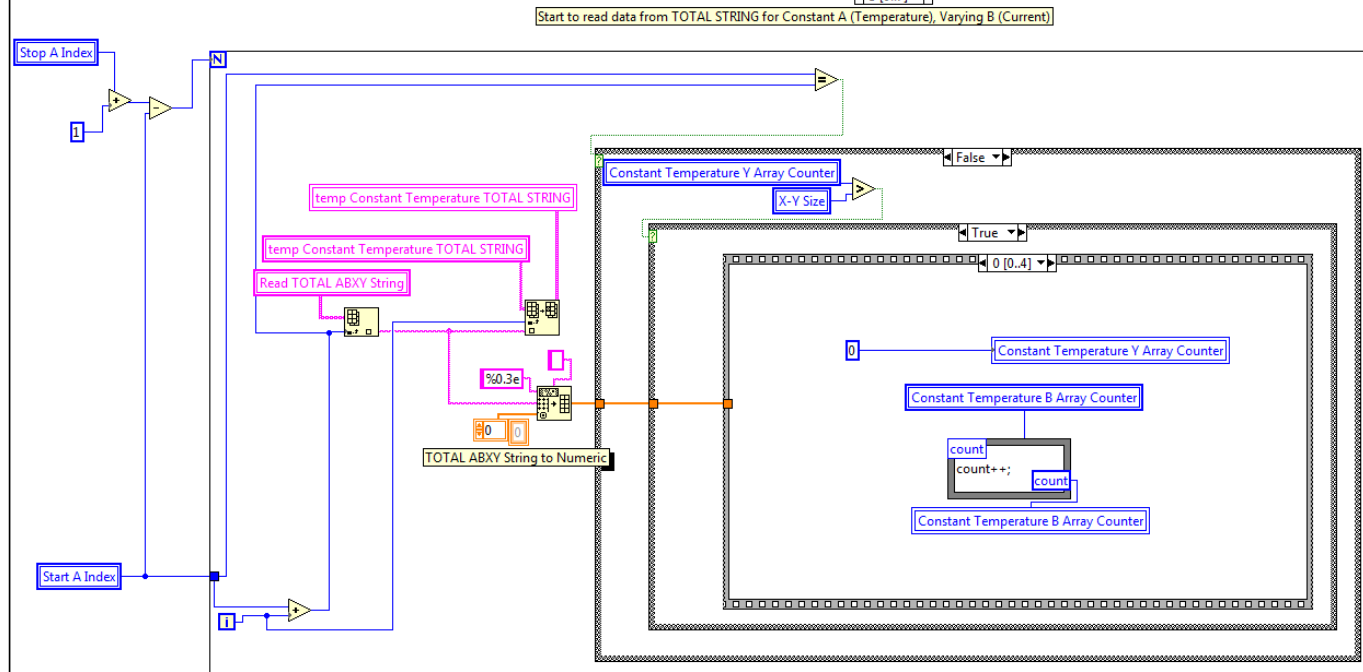

Read indexed.3.FALSE.TRUE.1

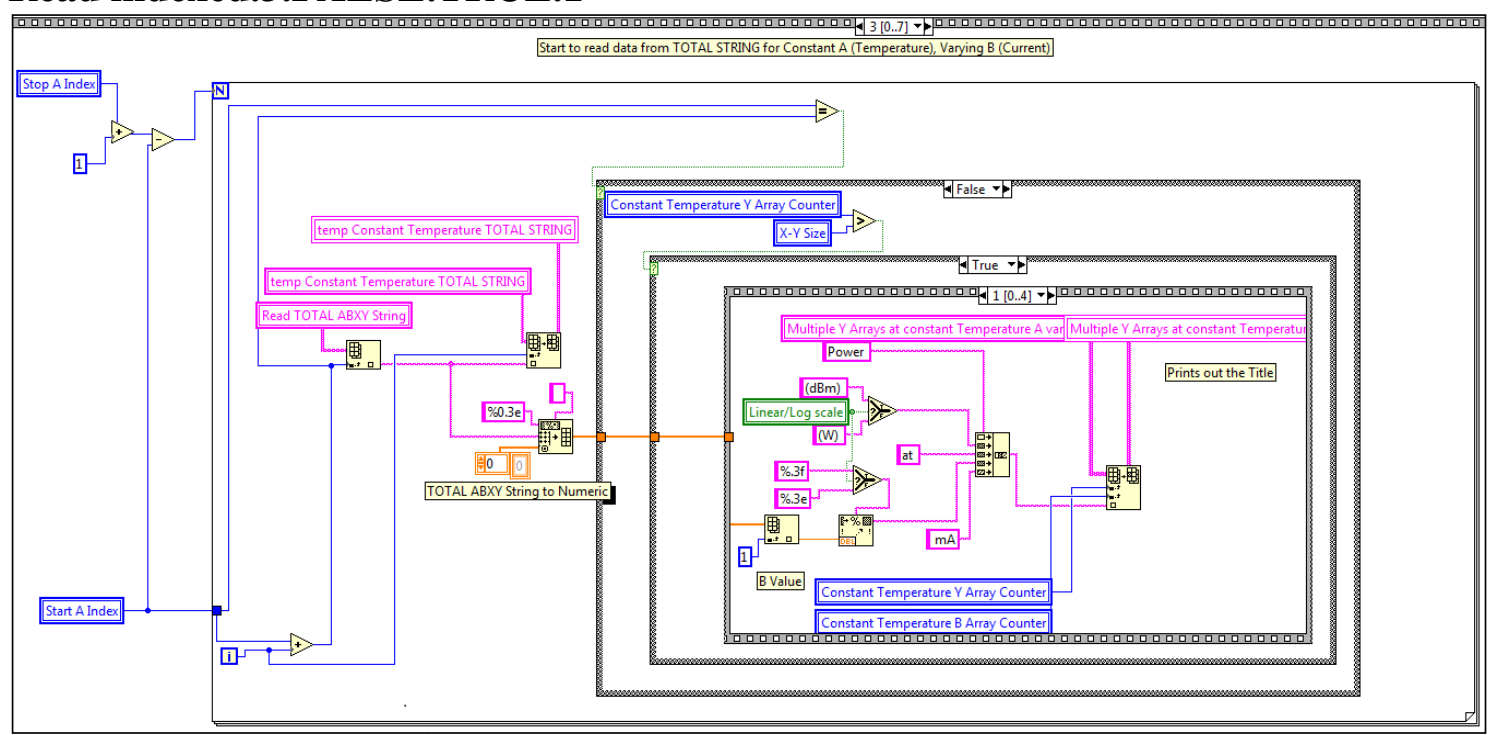




\section{Read indexed.3.FALSE.TRUE.2}

$0000000000000000000000000000000000000000000000000000000000000000043[0.7]+1000000000000000000000000000000000000000000$ Start to read data from TOTAL STRING for Constant A (Temperature), Varying B (Current]

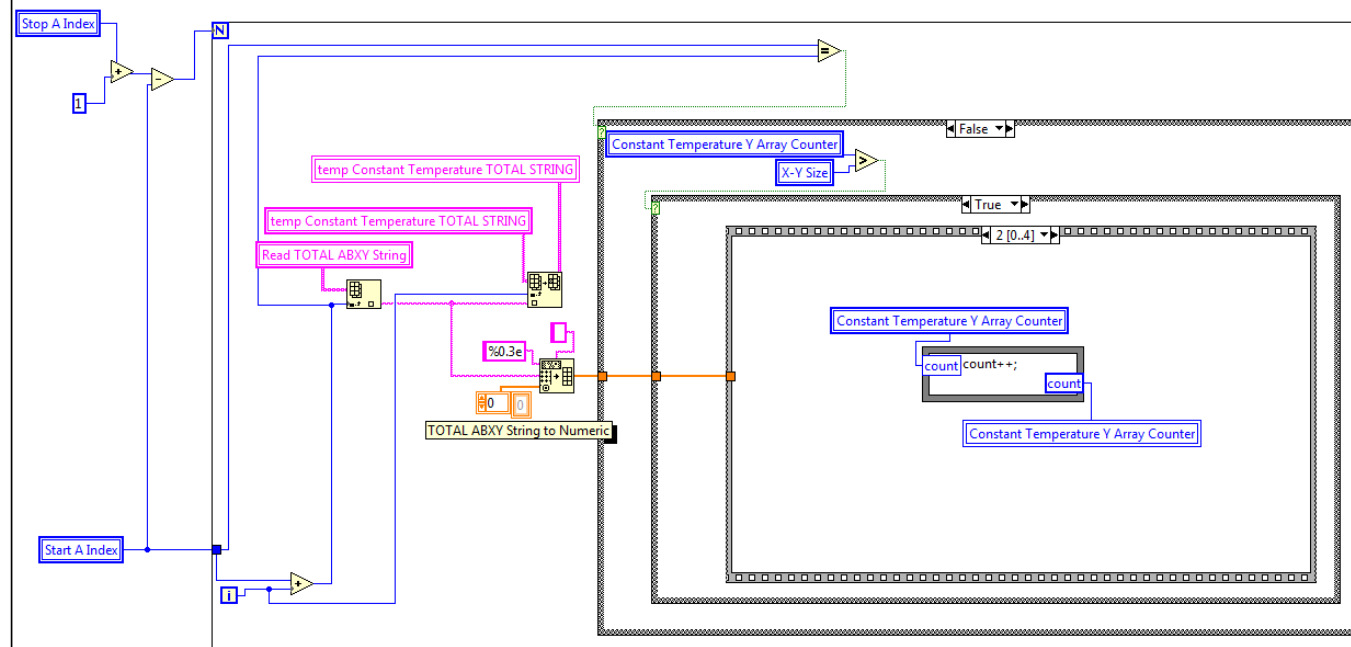

\section{Read indexed.3.FALSE.TRUE.3}

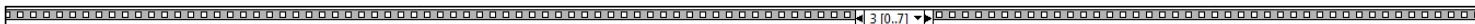

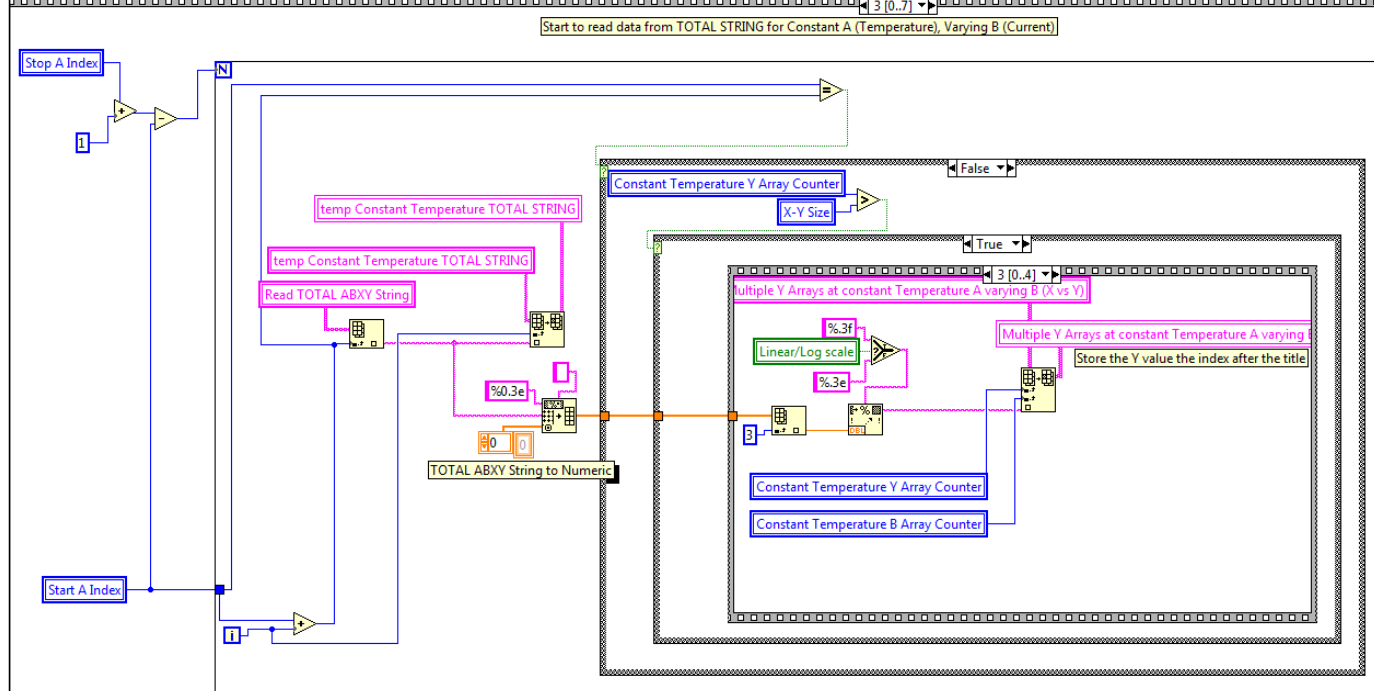




\section{Read indexed.3.FALSE.TRUE.4}

$00000000000000000000000000000000000000000000000000000000000000000000043[0.7]-10000000000000000000000000000000000000000000000000$ Start to read data from TOTAL STRING for Constant A (Temperature), Varying B (Current)
(T)

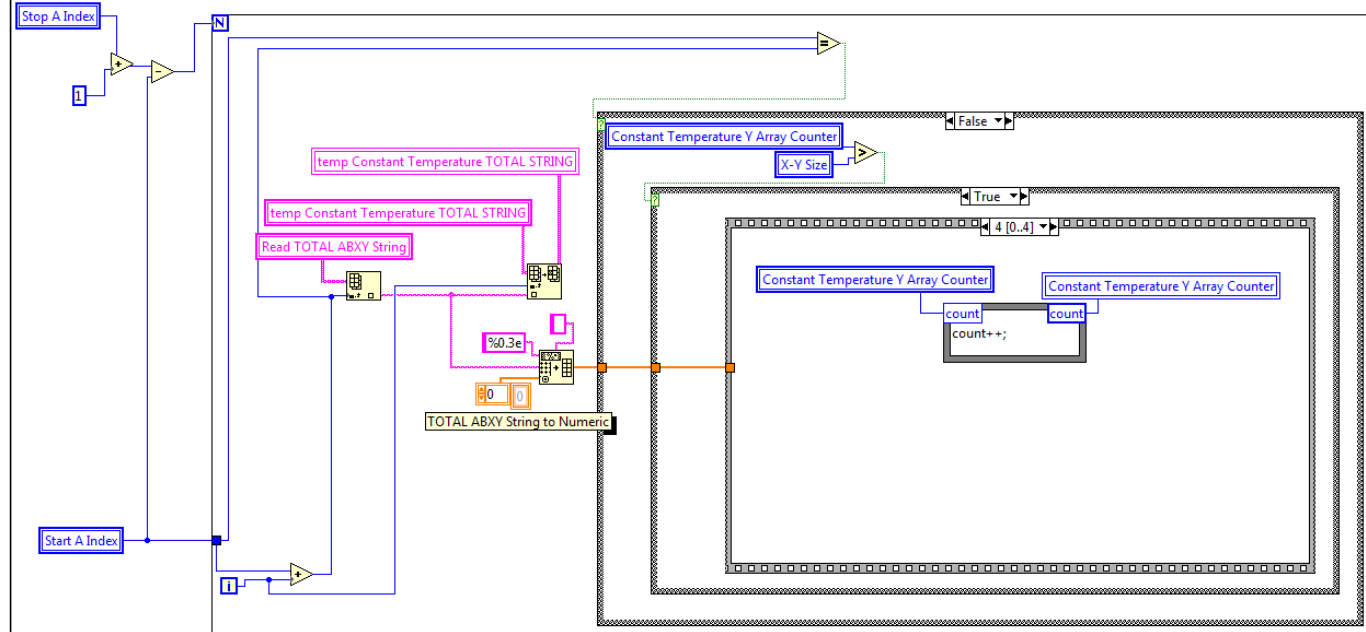

\section{Read indexed.3.TRUE.0}

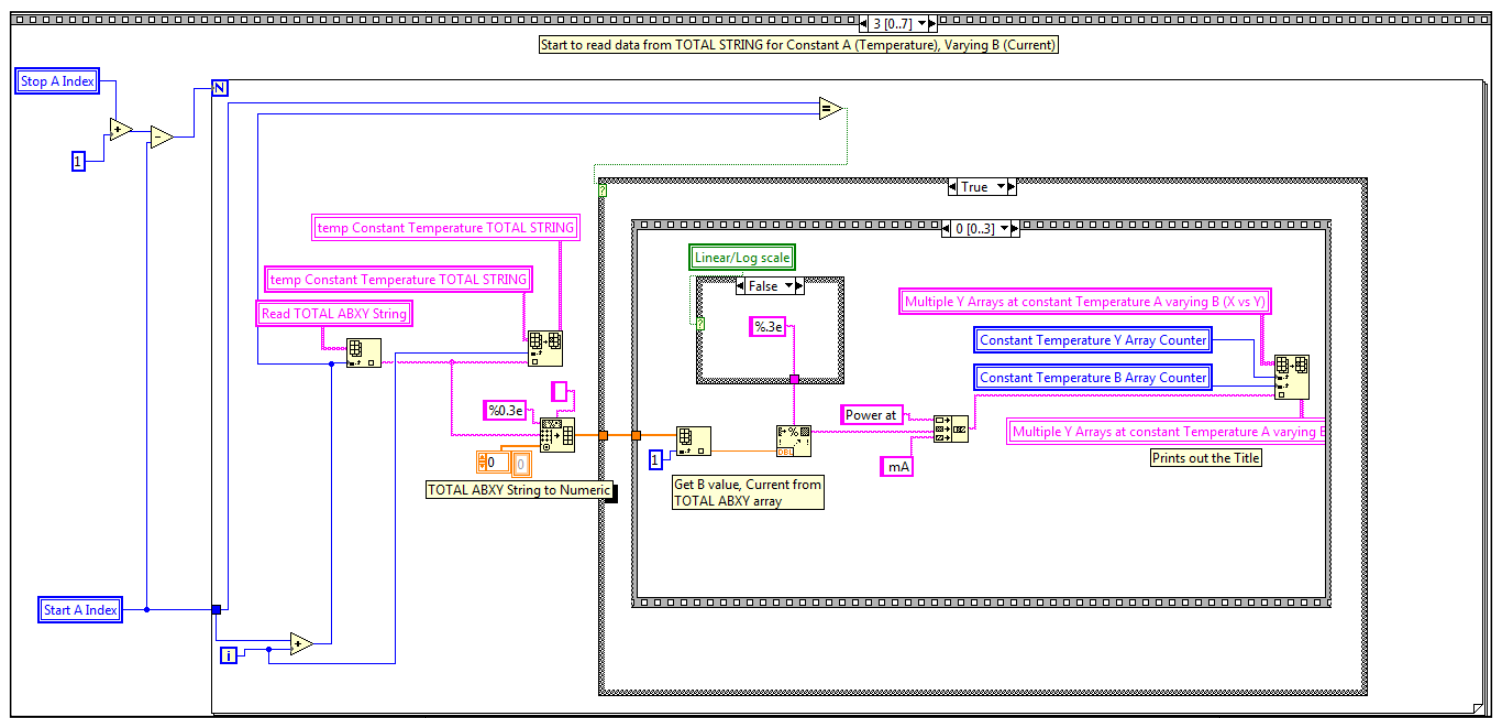




\section{Read indexed.3.TRUE.1}

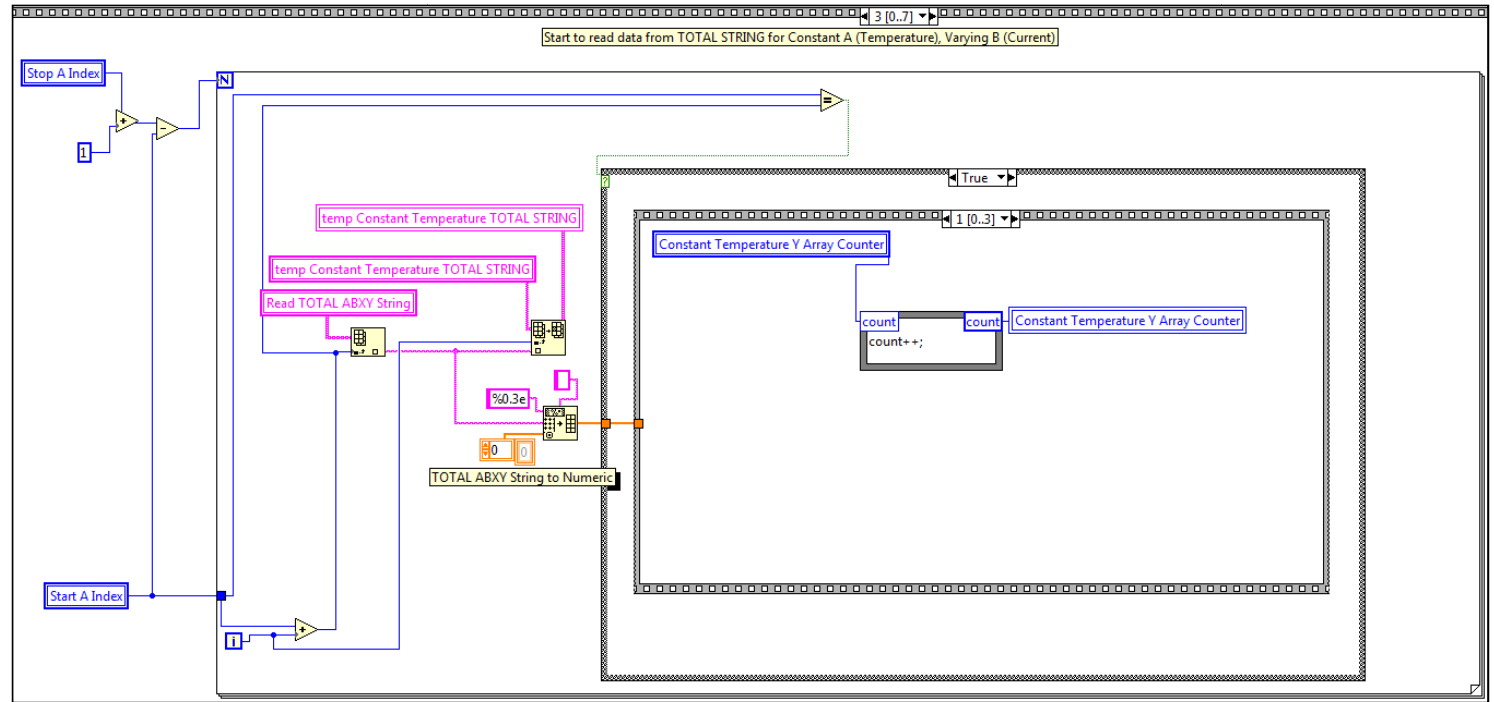

\section{Read indexed.3.TRUE.2}

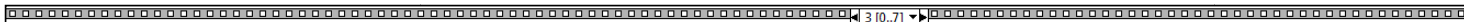

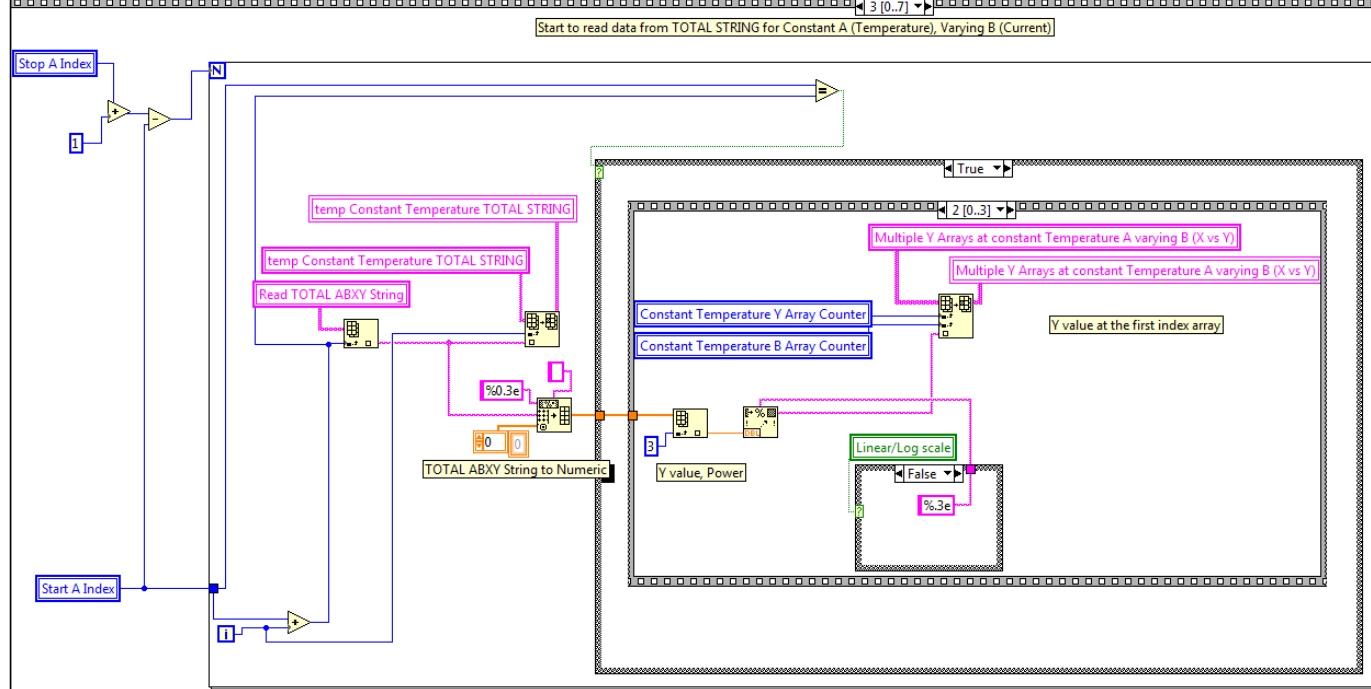




\section{Read indexed.3.TRUE.3}

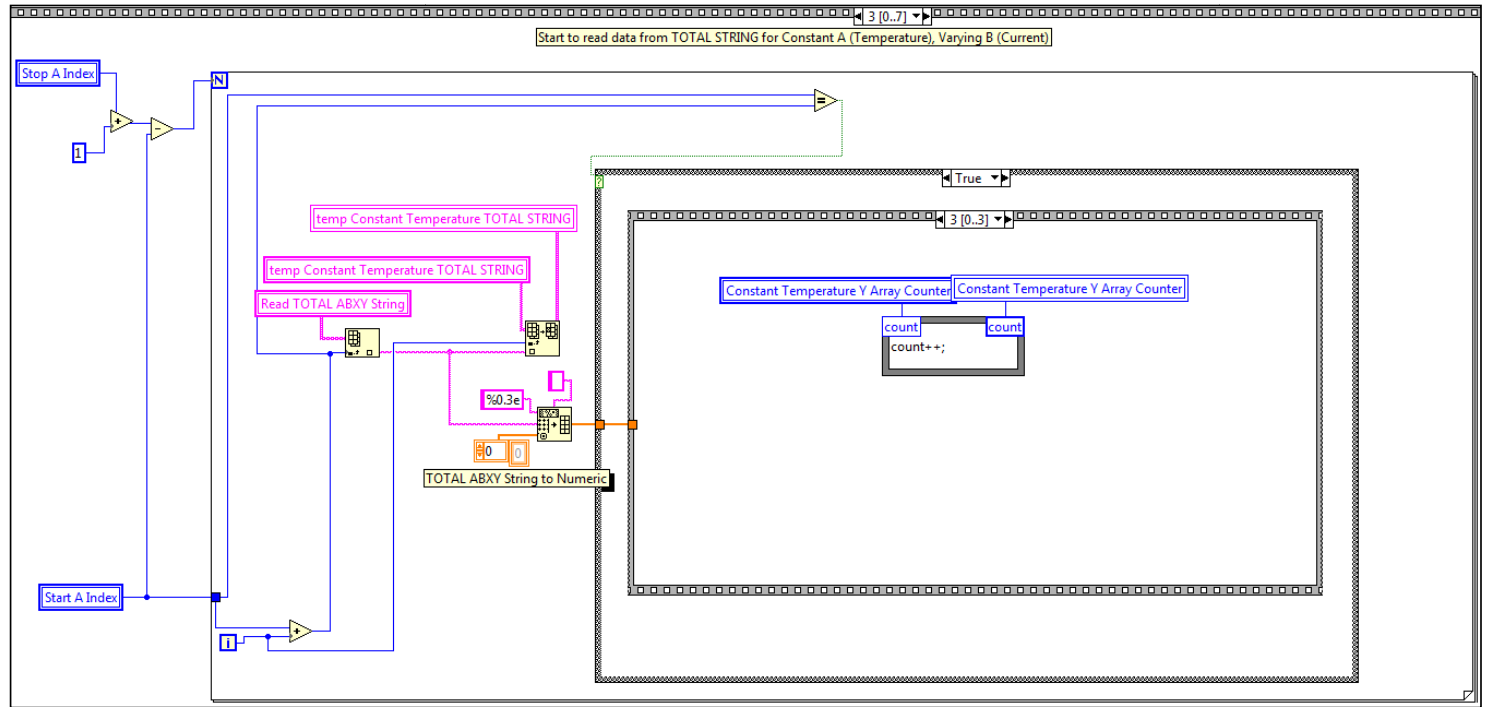

\section{Read indexed.4}

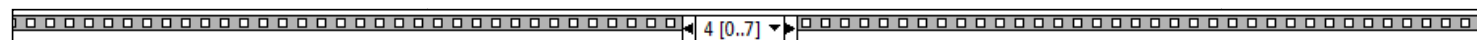

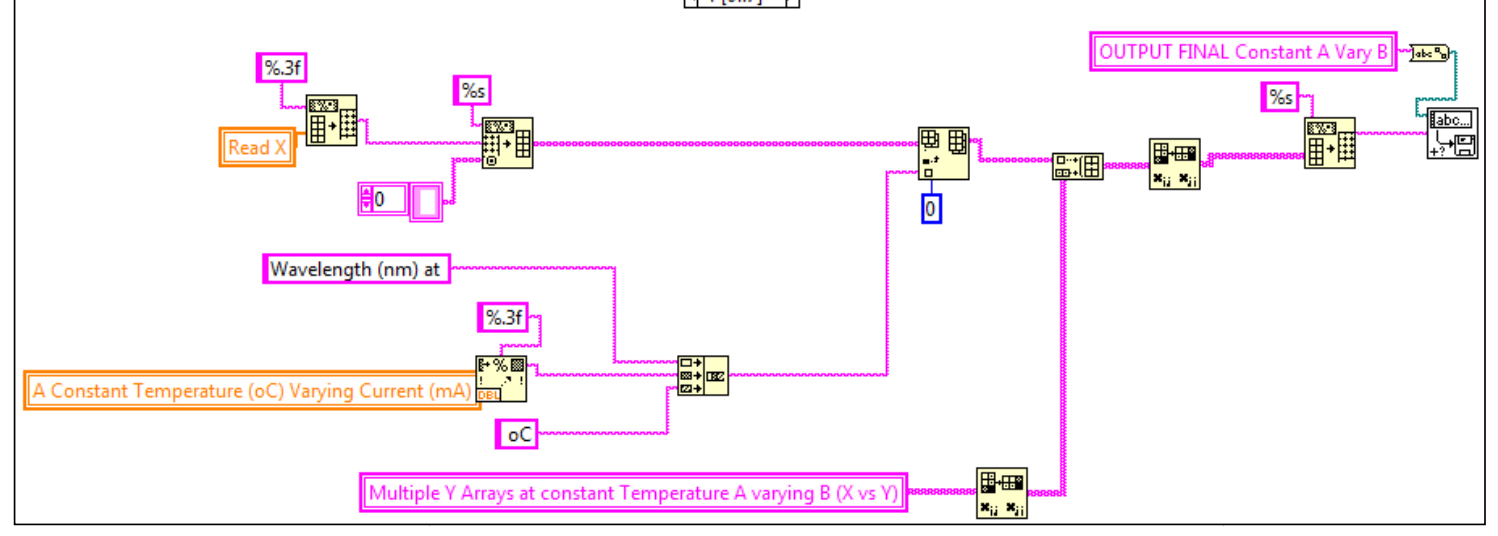




\section{Read indexed.5}

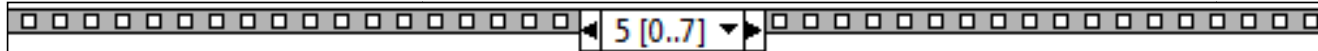

Initialize the values and arrays before generating Constant $\mathrm{B}$ with varying $\mathrm{A} x-\mathrm{y}$ plot
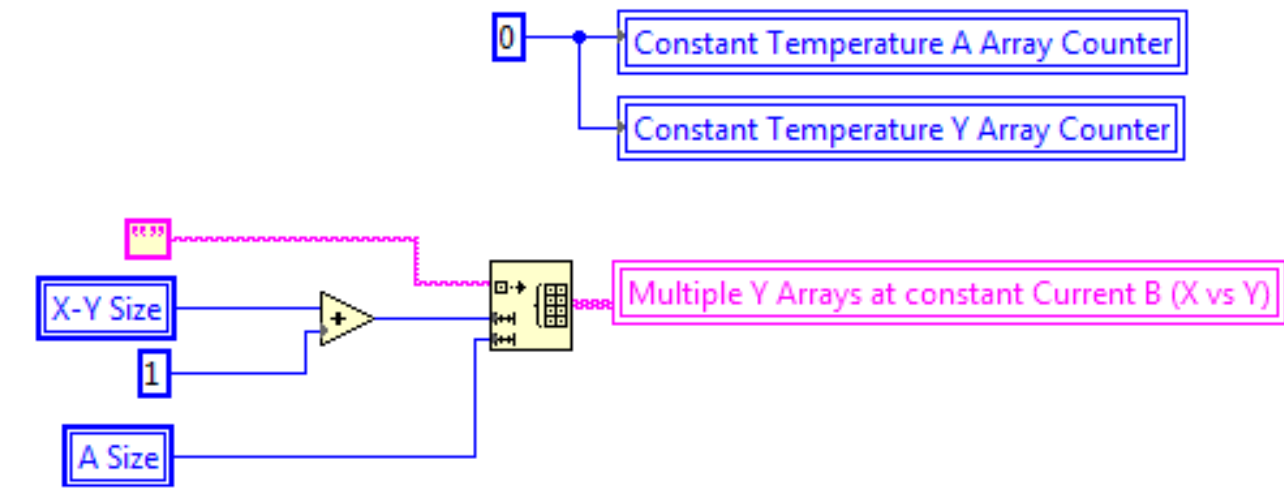

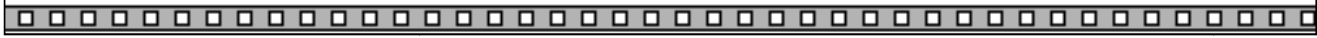

\section{Read indexed.6.FALSE.FALSE.0}

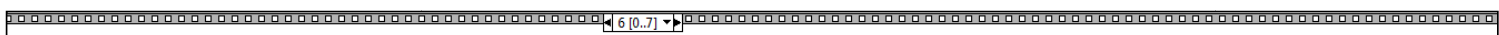
Generate Constant Input Current w/varying Temperature Array Output

That is the number of elements in $B$ Index Array, which is equivalent to how many input $A$ with a constant $B$

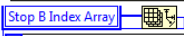

Stop B Index Array

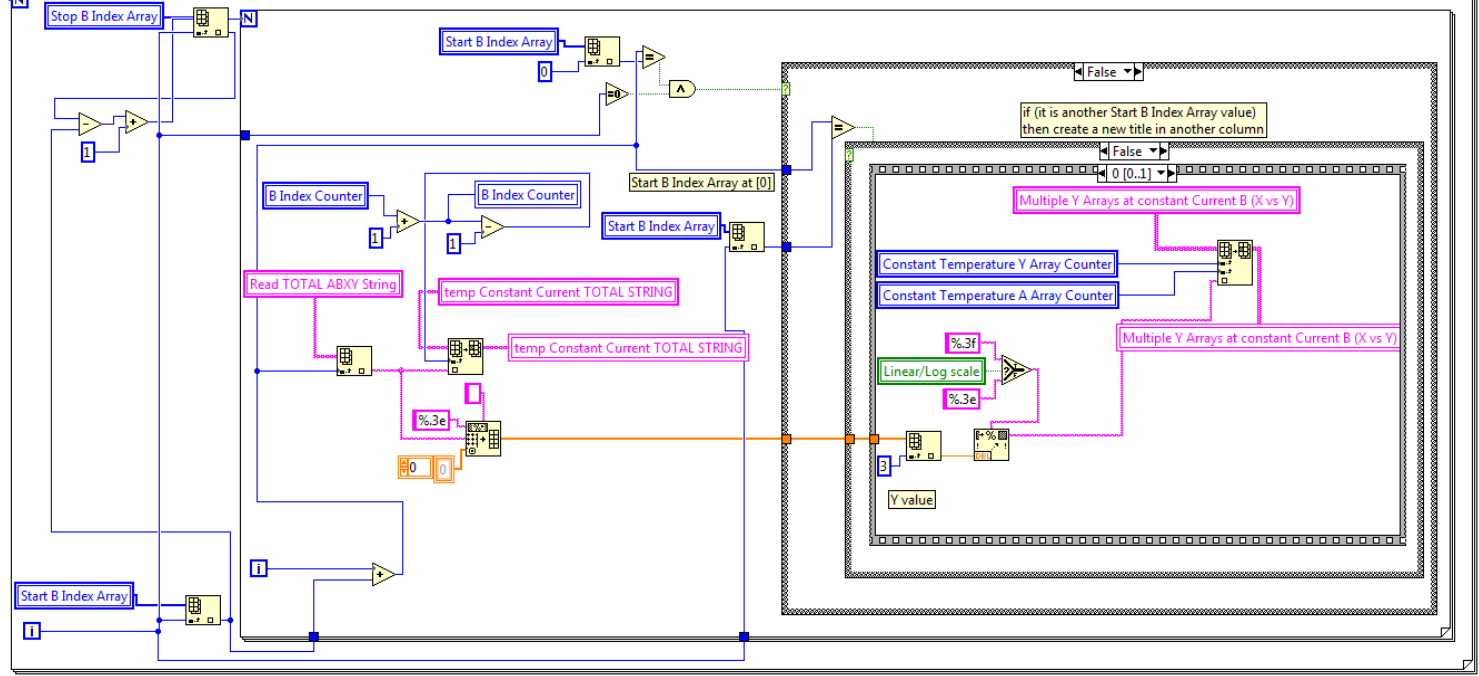




\section{Read indexed.6.FALSE.FALSE.1}

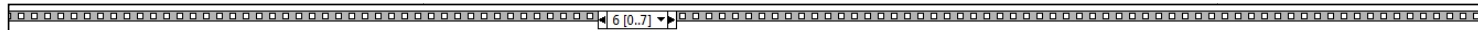
Generate Constant Input Current w/ varying Temperature Array Output

That is the number of elements in B Index Array, which is equivalent to how many input A with a constant $B$ Stop B Index Array

N 5 Stop B Index Array

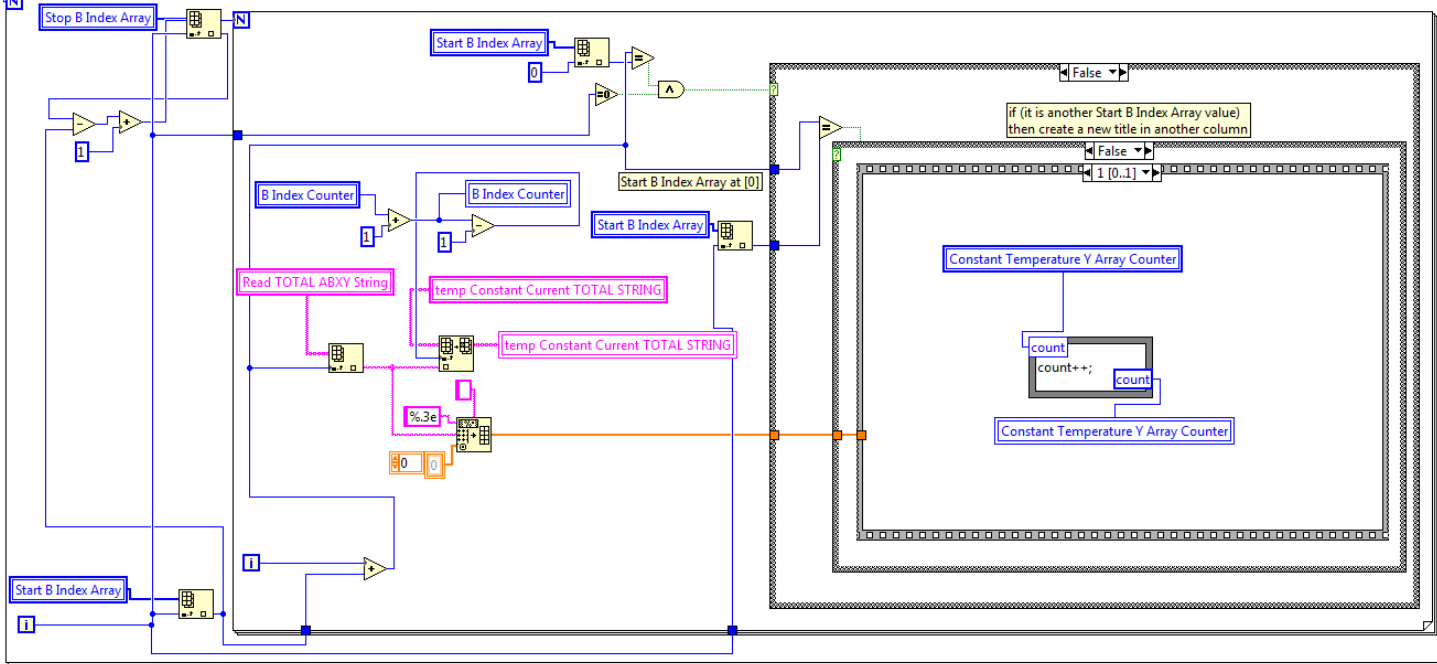

\section{Read indexed.6.FALSE.TRUE.0}

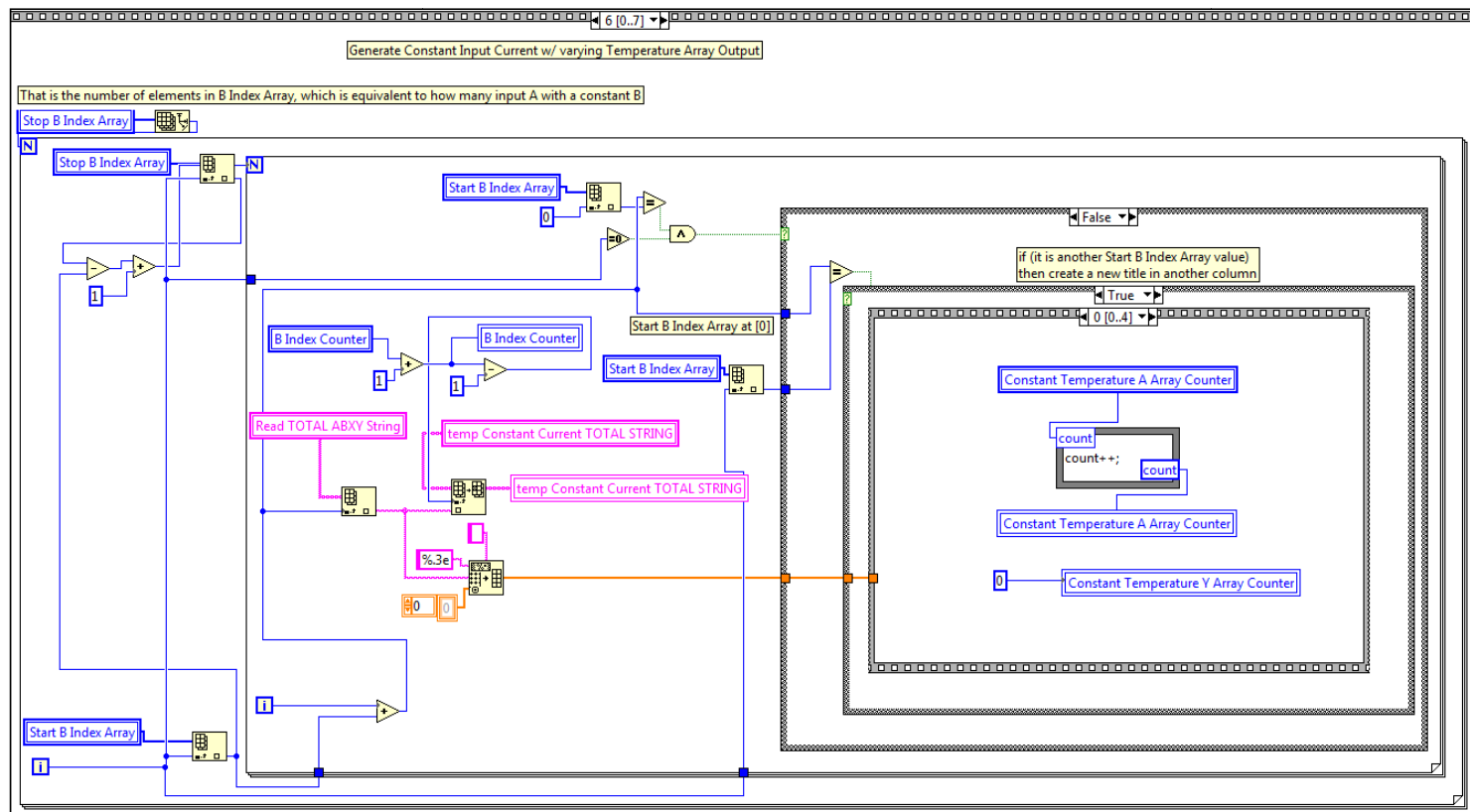




\section{Read indexed.6.FALSE.TRUE.1}

$0000000000000000000000000000000000000000000046[0.7]+80000000000000000000000000000000000000000000000000000000000000$ Generate Constant Input Current w/ varying Temperature Array Output

That is the number of elements in B Index Array, which is equivalent to how many input $A$ with a constant $B$ Stop B Index Array

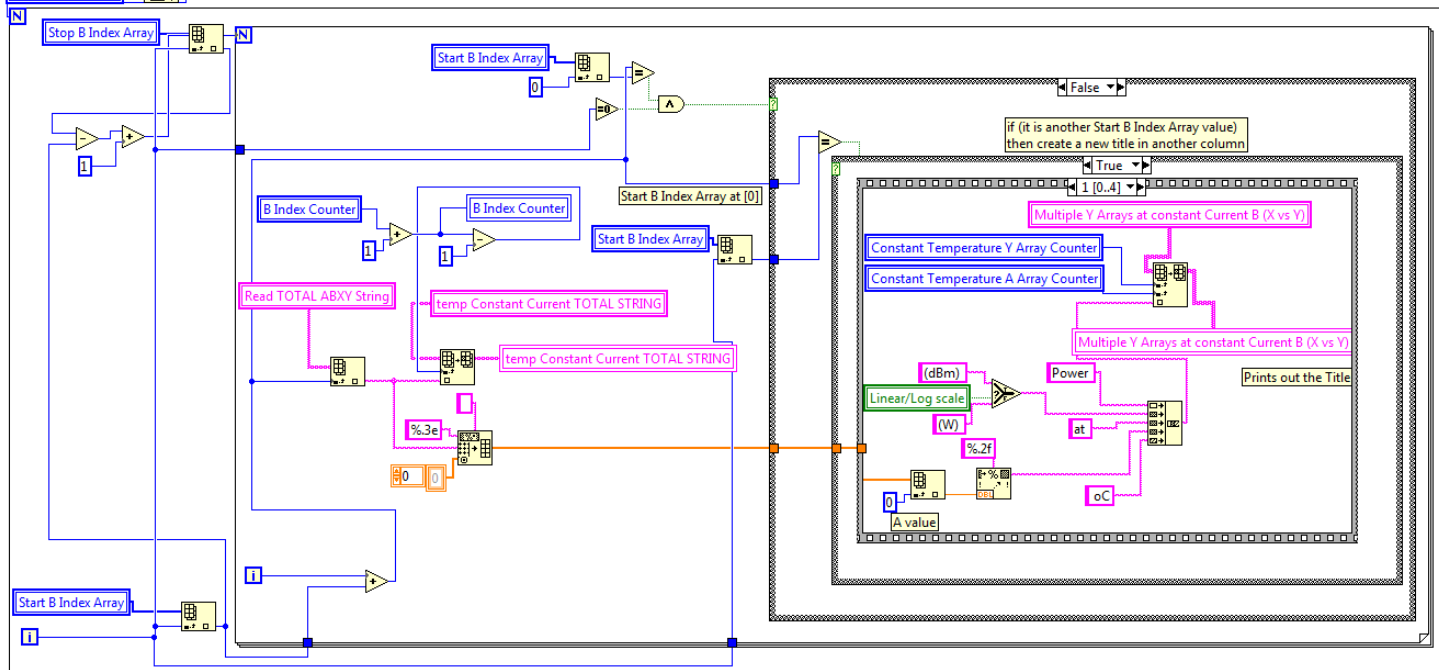

\section{Read indexed.6.FALSE.TRUE.2}

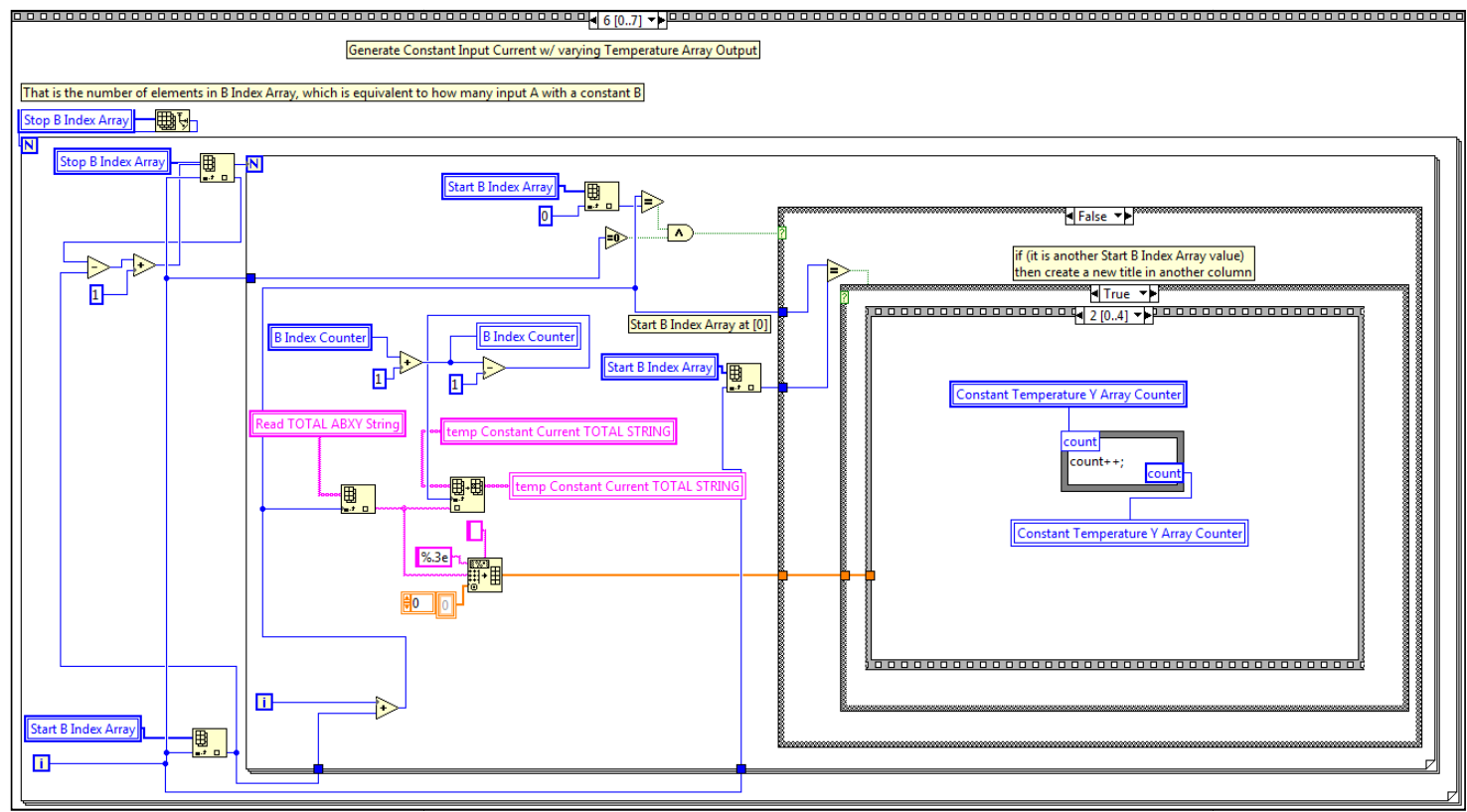




\section{Read indexed.6.FALSE.TRUE.3}

$0000000000000000000000000000000000000000000004600.7]+80000000000000000000000000000000000000000000000000000000000000$ Generate Constant Input Current w/varying Temperature Array Output

That is the number of elements in $B$ Index Array, which is equivalent to how many input $A$ with a constant $B$

Stop B Inder Array -

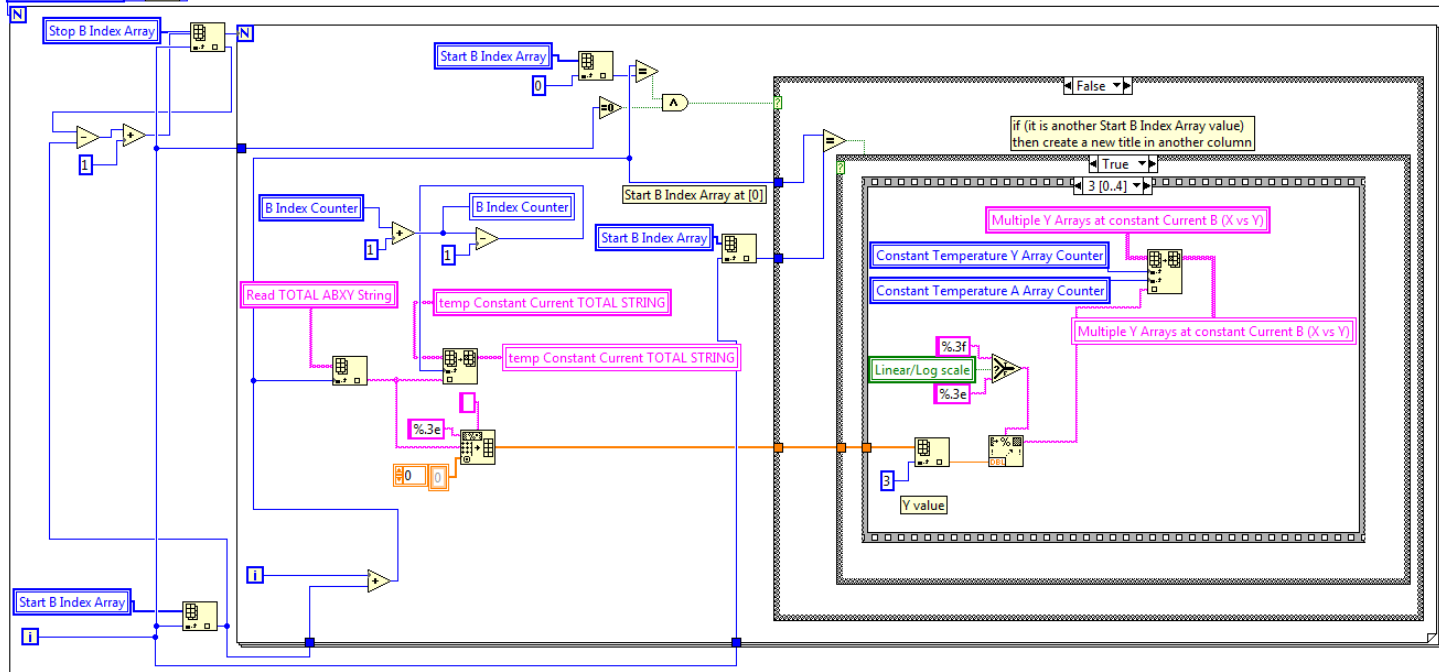

\section{Read indexed.6.FALSE.TRUE.4}

$\frac{0}{0000000000000000000000000000000000000000000046[0.7]+10000000000000000000000000000000000000000000000000000000000000}$ Generate Constant Input Current w/ varying Temperature Array Output

That is the number of elements in $B$ Index Array, which is equivalent to how many input $A$ with a constant $B$

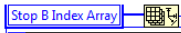

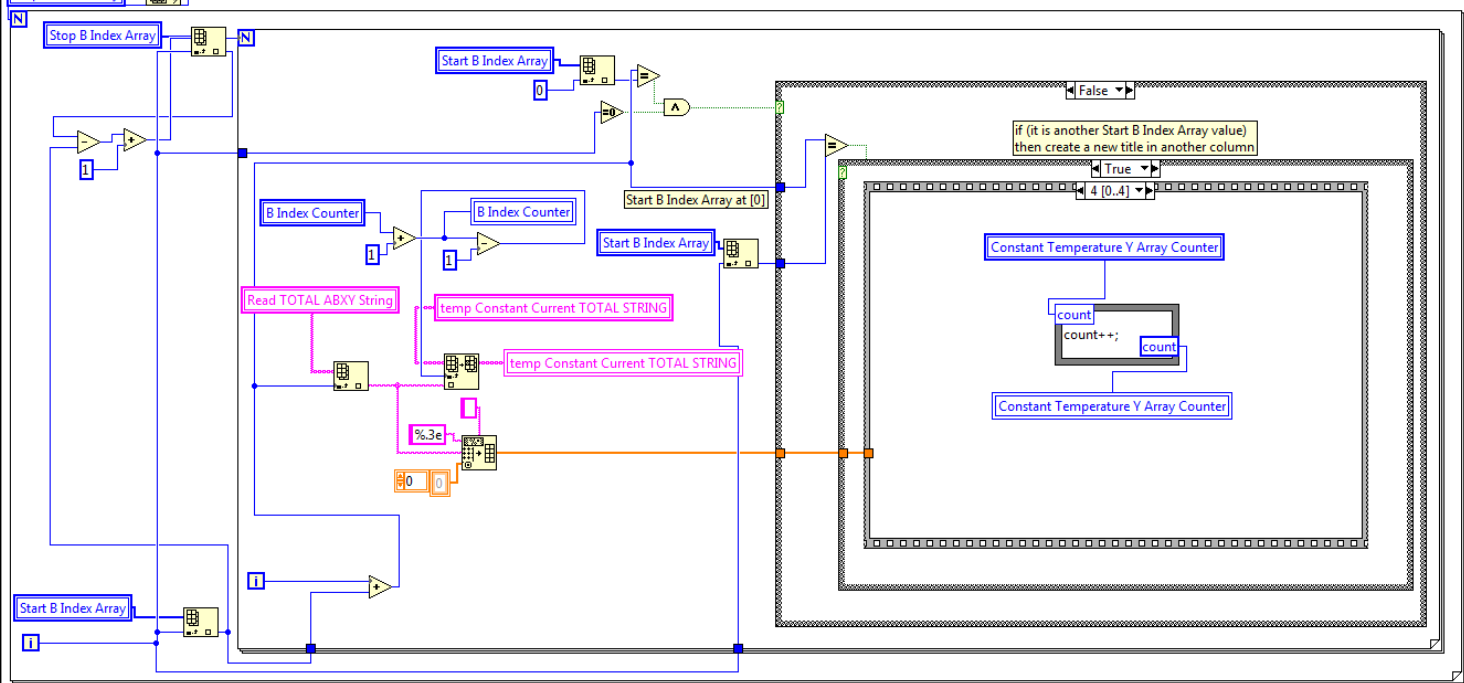




\section{Read indexed.6.TRUE.0}

0000000000000000000000000000000000000000000046[0.7] 60000000000000000000000000000000000000000000000000000000000000 Generate Constant Input Current w/varying Temperature Array Output

That is the number of elements in $B$ Index Array, which is equivalent to how many input $A$ with a constant $B$

Stop B Index Array

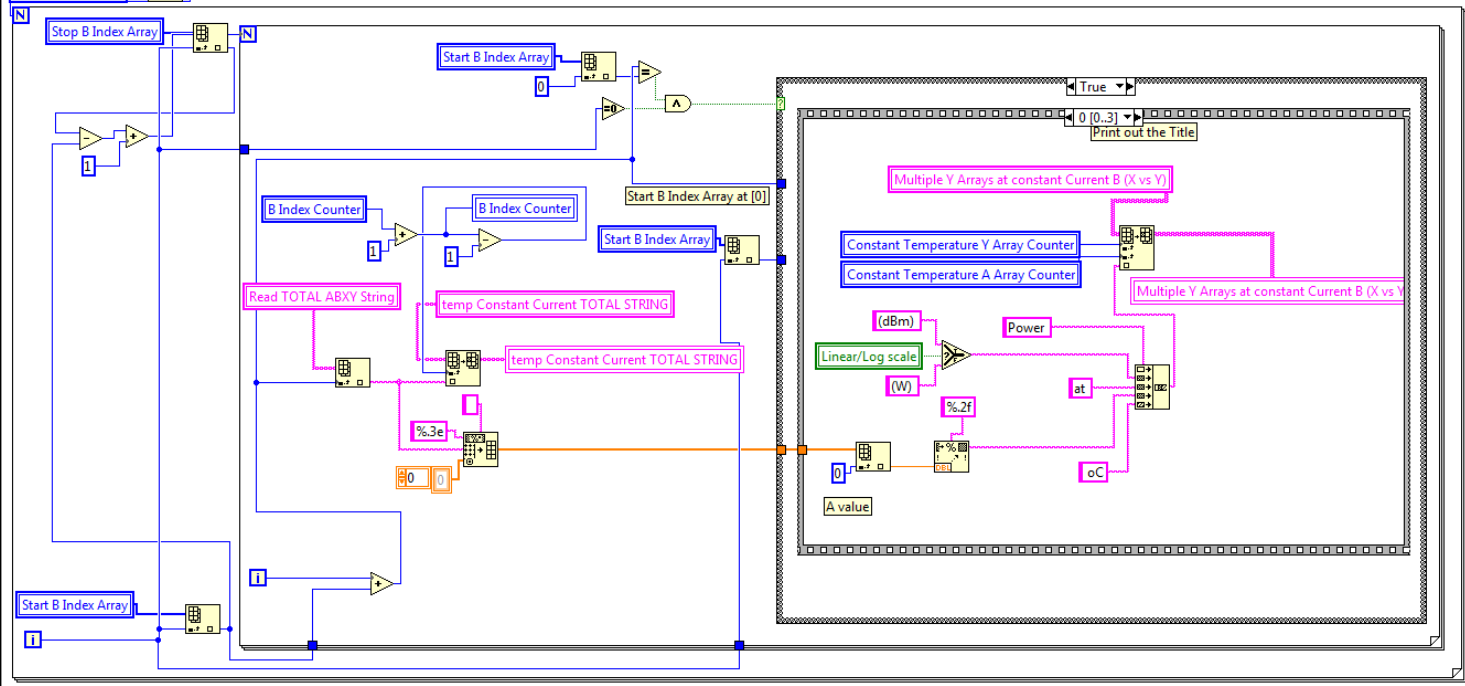

\section{Read indexed.6.TRUE.1}

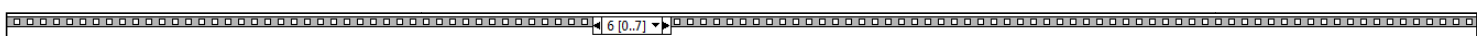
Generate Constant Input Current w/varying Temperature Array Output

That is the number of elements in B Index Array, which is equivalent to how many input $A$ with a constant $B$

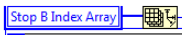

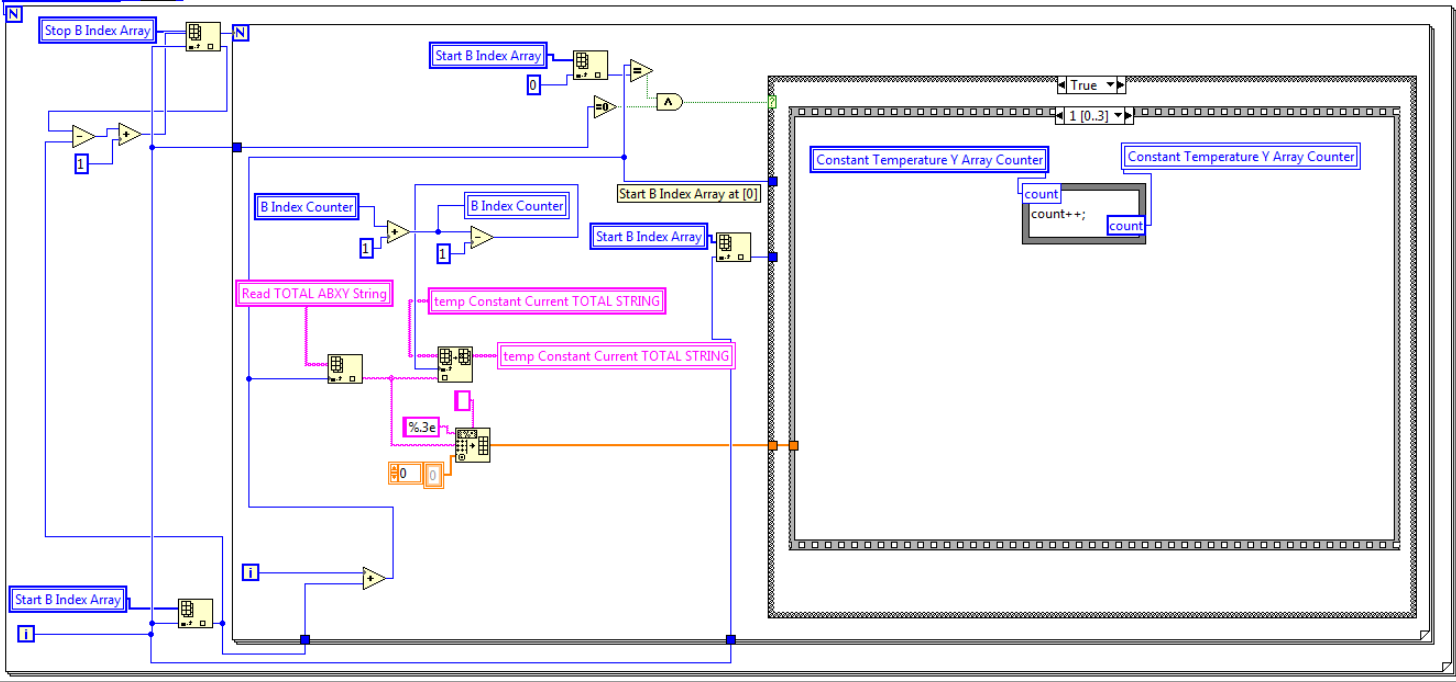




\section{Read indexed.6.TRUE.2}

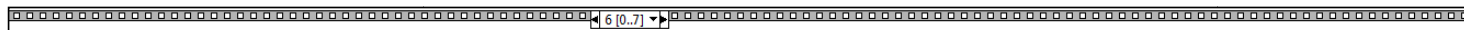
Generate Constant Input Current w/varying Temperature Array Output

That is the number of elements in $B$ Index Array, which is equivalent to how many input $A$ with a constant $B$

Stop B Index Array - 傮泡,

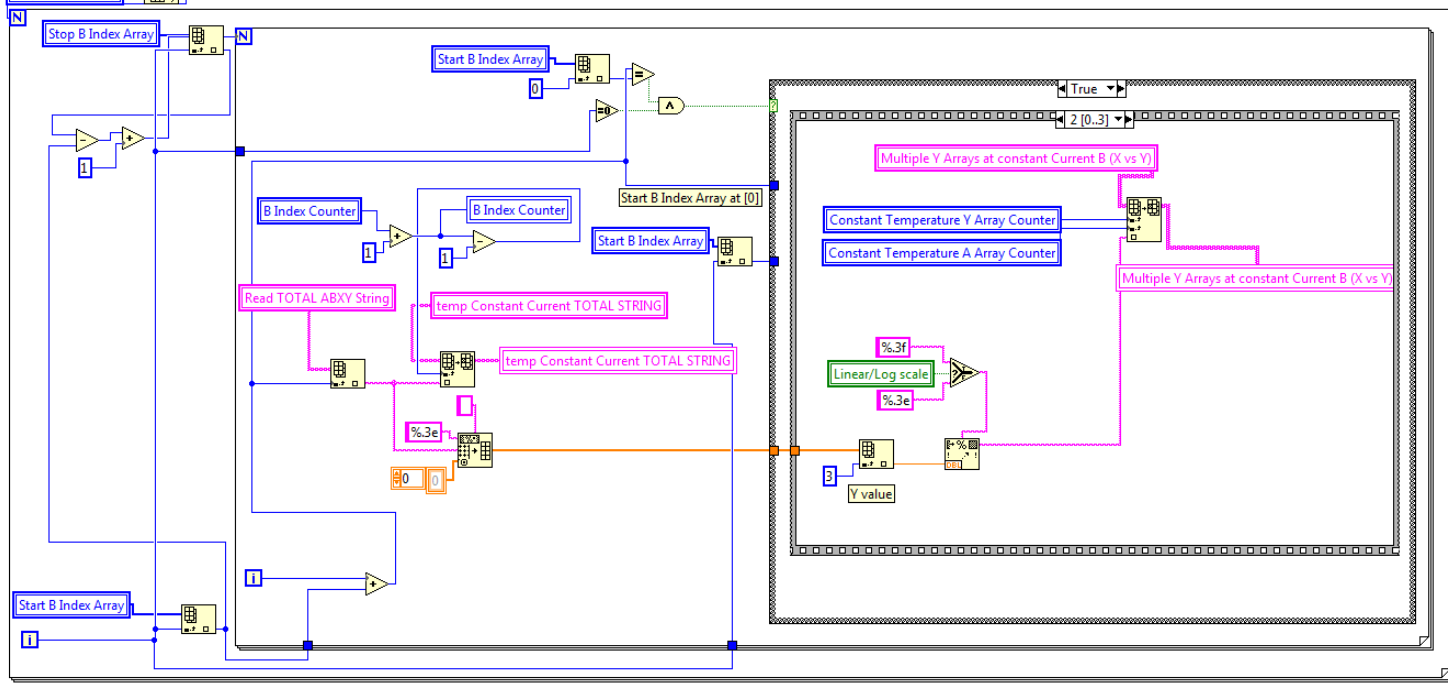

\section{Read indexed.6.TRUE.3}

1000000000000000000000000000000000000000000046[0.7] 180000000000000000000000000000000000000000000000000000000000000 Generate Constant Input Current w/varying Temperature Array Output

That is the number of elements in B Index Array, which is equivalent to how many input A with a constant B

Stop B Index Array -

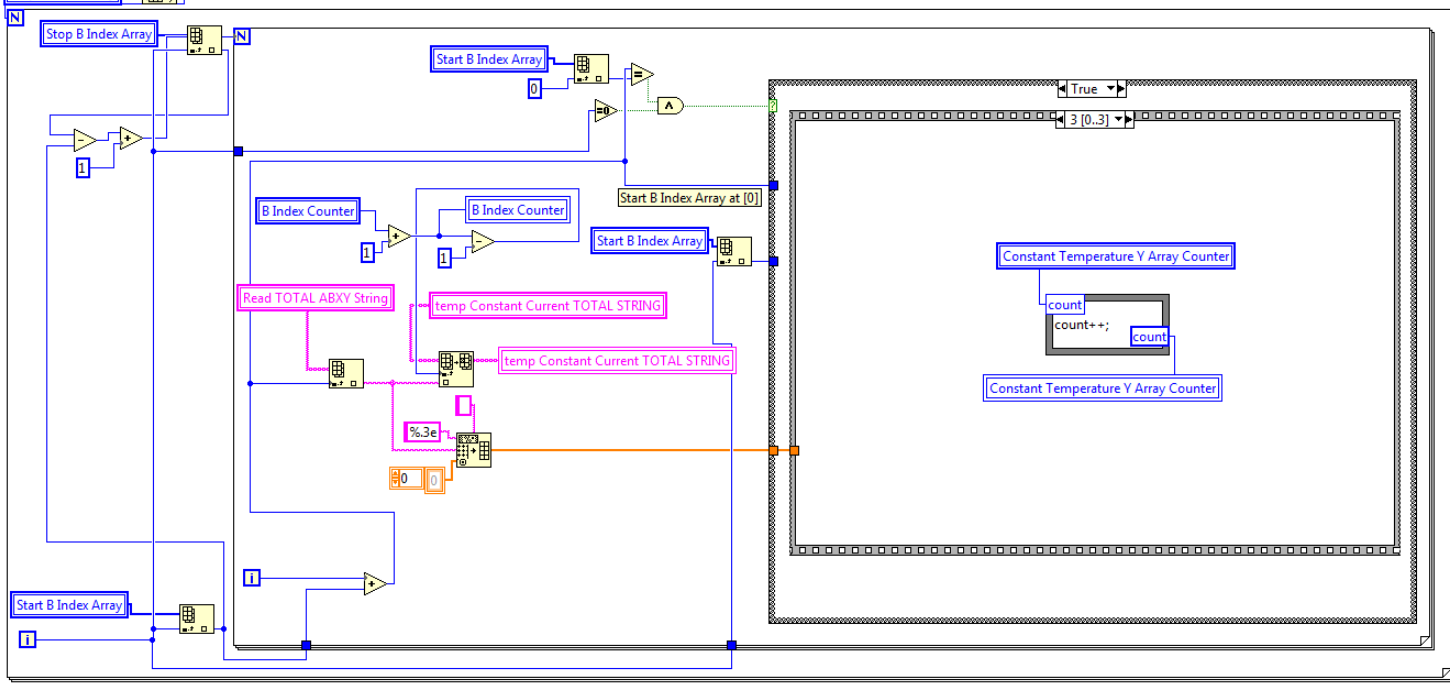




\section{Read indexed.7.FALSE}

$00000000000000000000000000000000000497[0.7]+10000000000000000000000000000000000000000$

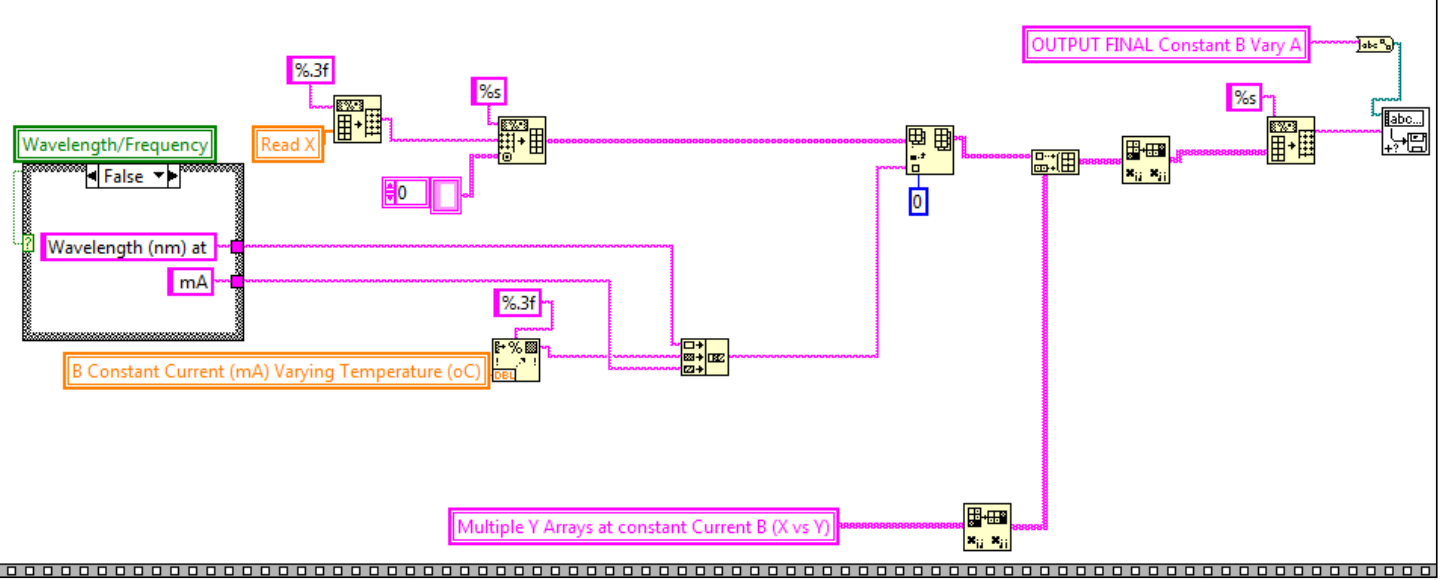

\section{Read indexed.7.TRUE.cropped}

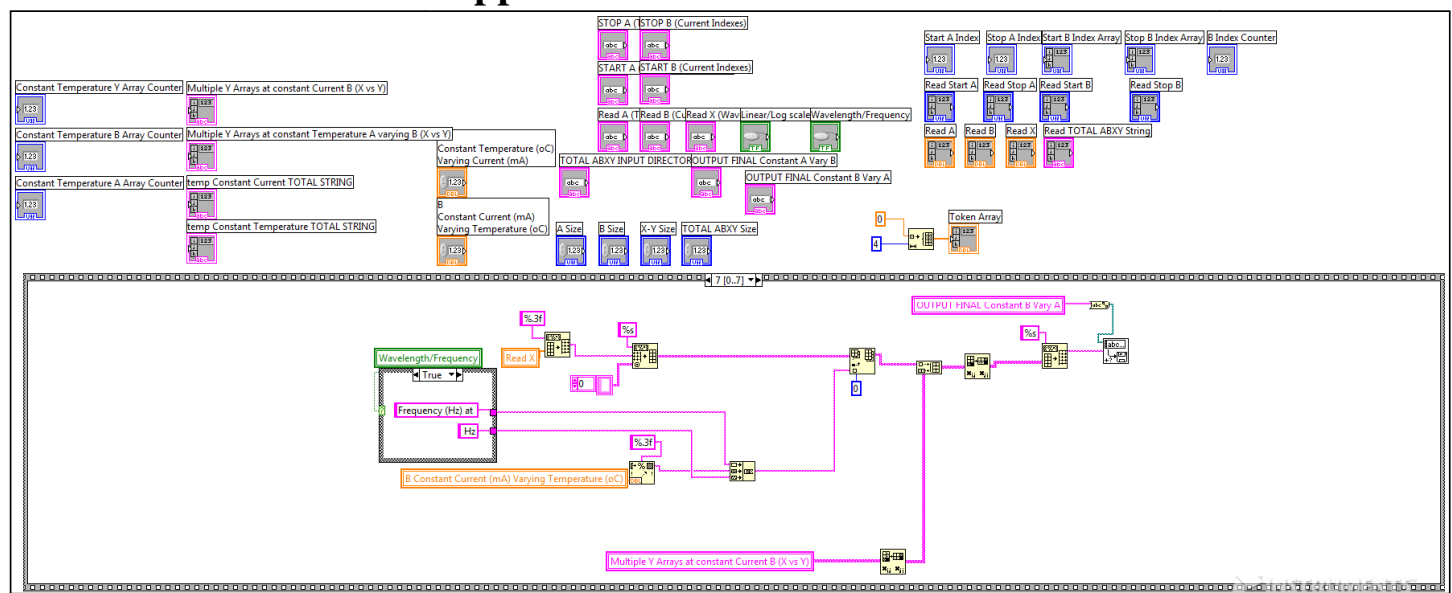




\section{APPENDIX C: Experiment Pictures}

Fabry-Pérot (FP) Lasers at 1300 nm:
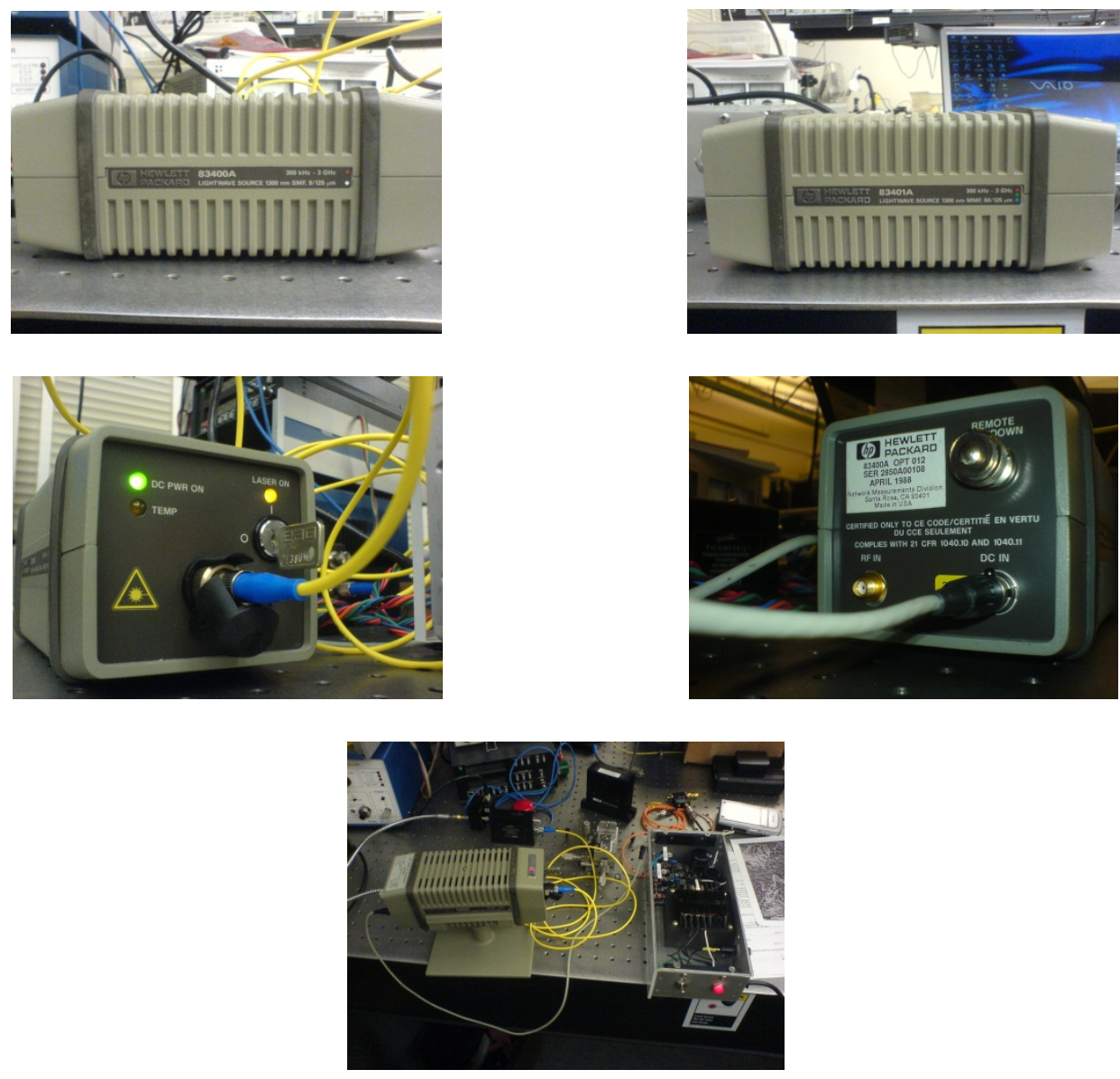

FP laser with Power Supply HCBB-75W-A

Distributed Feedback (DFB) Laser at $1550 \mathrm{~nm}$ :

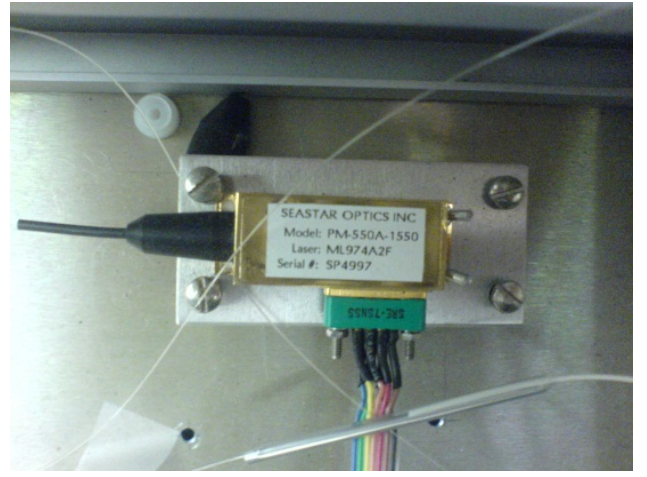


Vertical-Cavity Surface-Emitting Laser (VCSEL) at $850 \mathrm{~nm}$ :

Honeywell HFE4192-901, 4.25 Gbps 850 nm VCSEL LC TOSA:
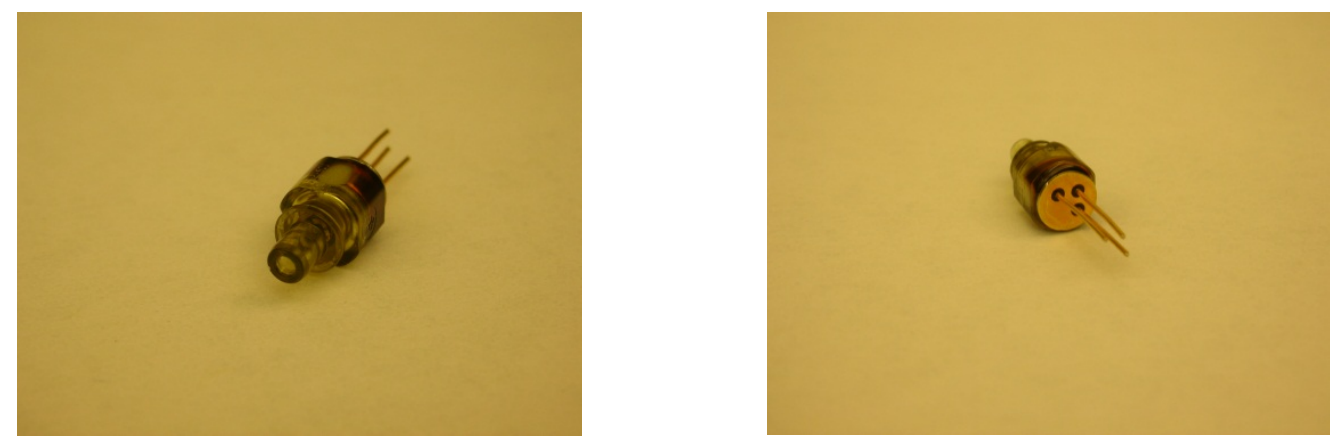

Low-Power VCSEL at $850 \mathrm{~nm}$ :
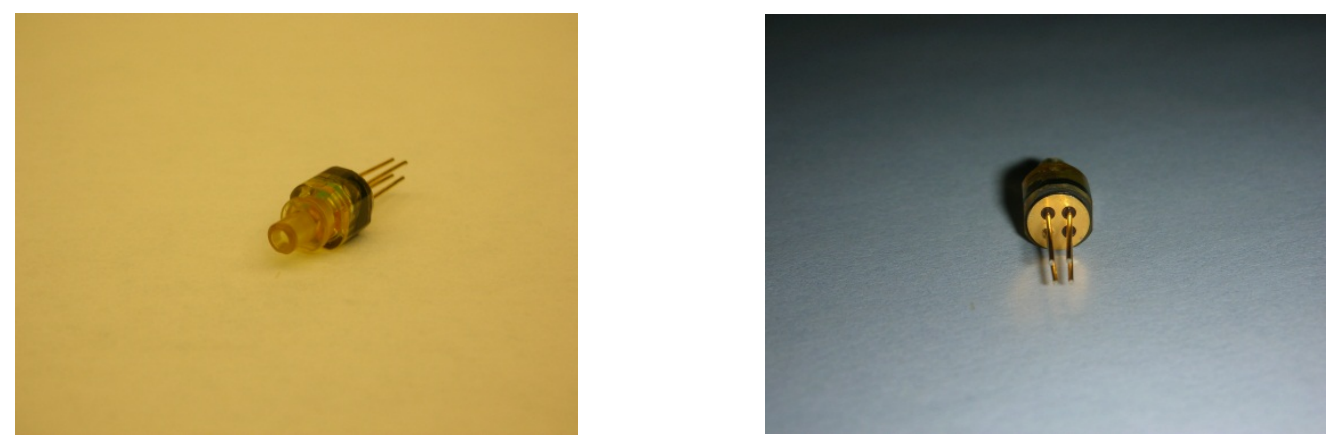

High-Power VCSEL at $850 \mathrm{~nm}$ :
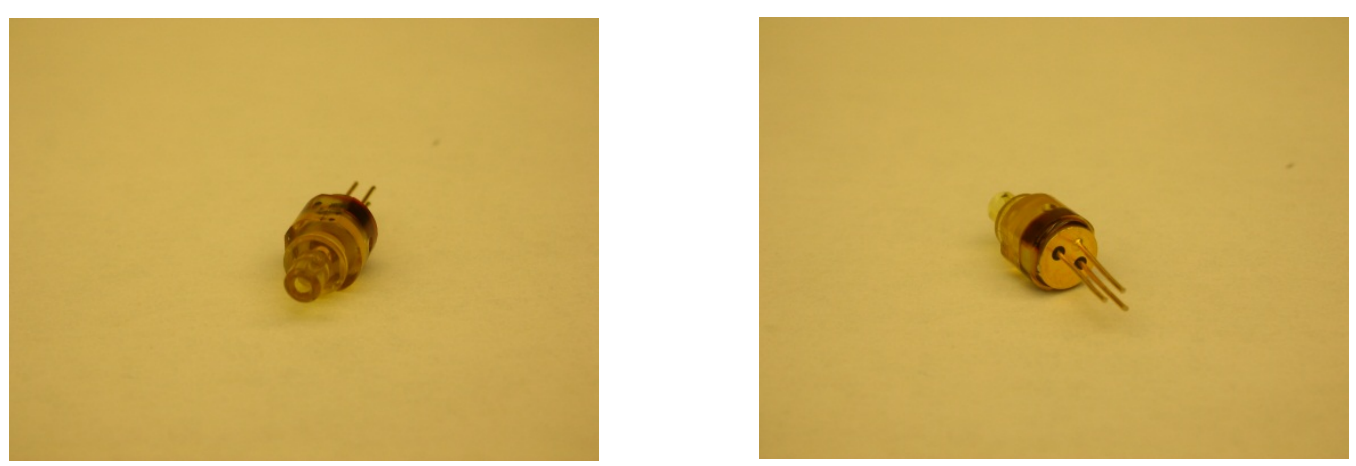
DC L-V-I Cuve Measument:

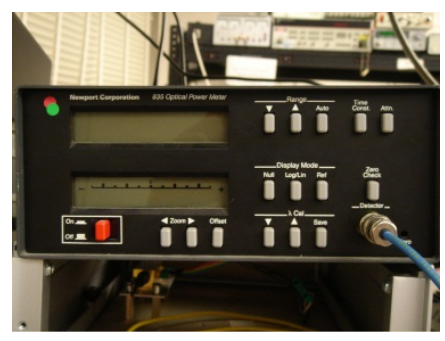

Newport Power Meter 835

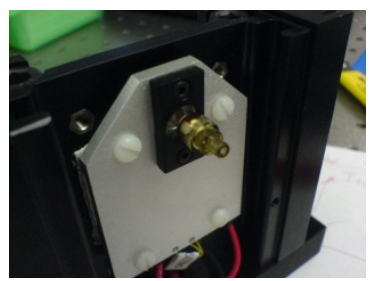

ILX Lightwave LDM-4407 Temperature Controller Mount with VCSEL

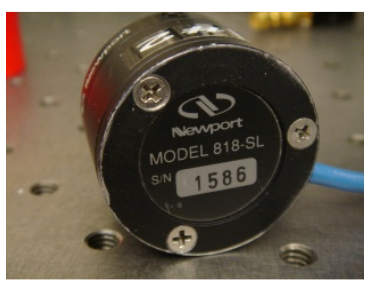

Newport Photodetector 818-SL

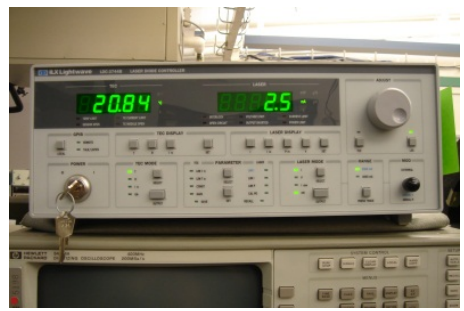

ILX-Lightwave Laser Diode Controller LDC-3744B

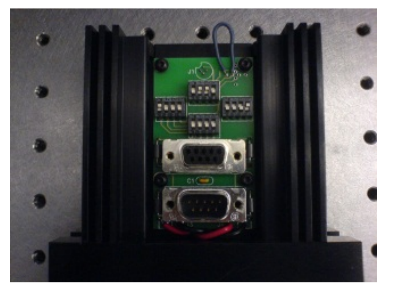

ILX Lightwave LDM-4407 Temperature Controller Mount (Rear with config. ports)

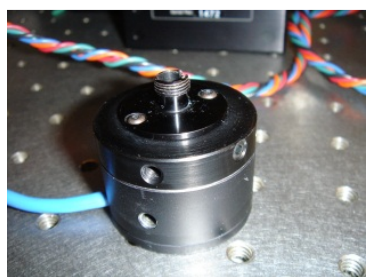

Newport Photodetector 818-SL (with laser connector)

DC Optical Spectrum Measurement:

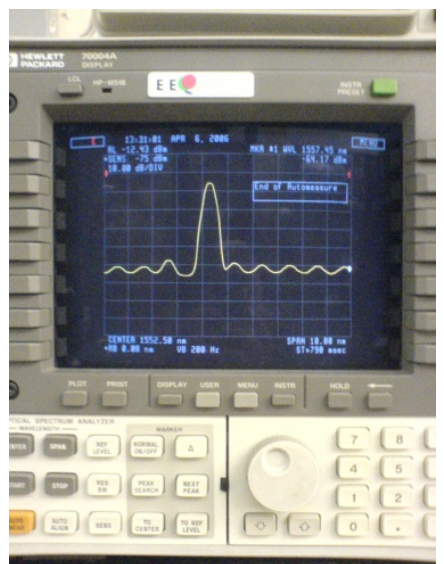

HP 71450A Optical Spectrum Analyzer 
Bandwidth (Frequency Response) Measurement:

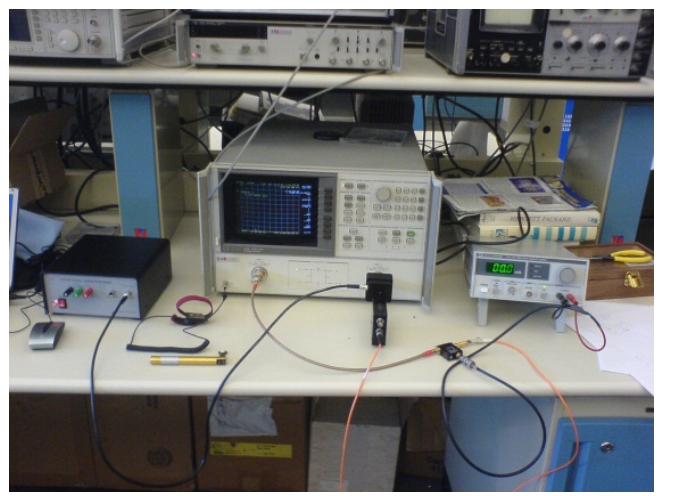

Bandwidth Measurement Experiment Setup with HP 8720B Network Analyzer

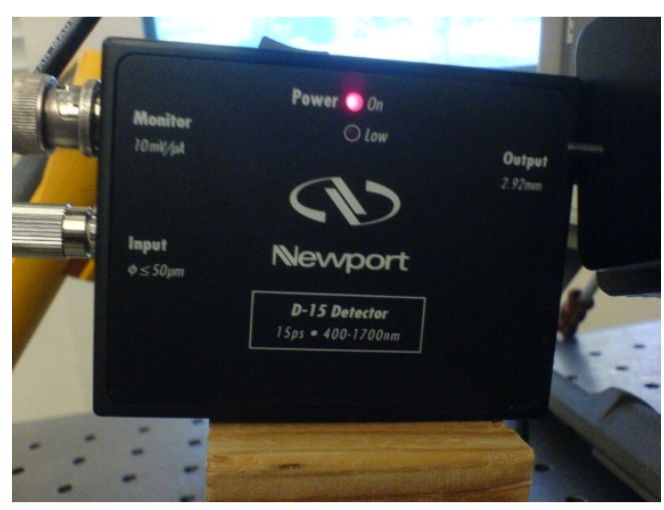

Newport D-15 Hi-Speed Photo Detector

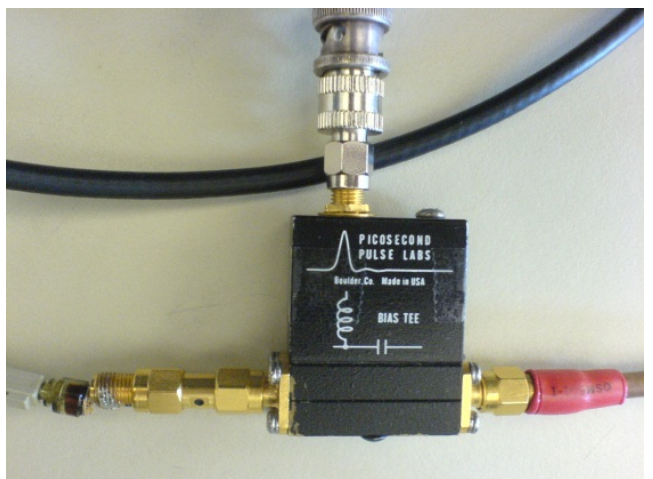

Picosecond Pulse Labs Bias-Tee

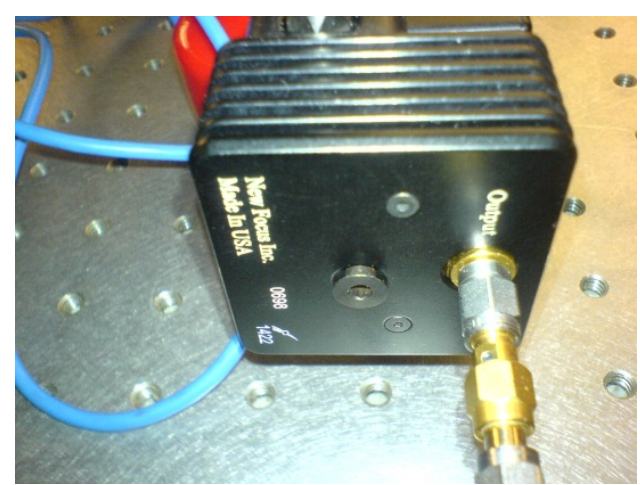

New Focus 1422 Broadband Amplifier 
Relative Intensity Noise (RIN) Measurement:

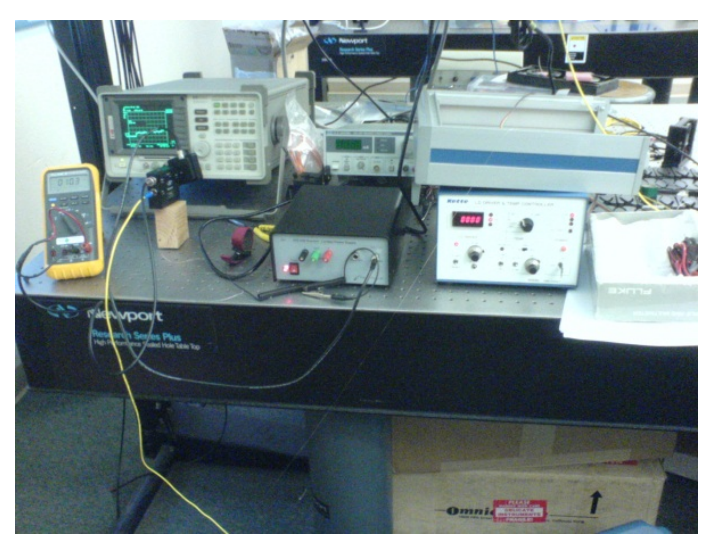

RIN experiment setup

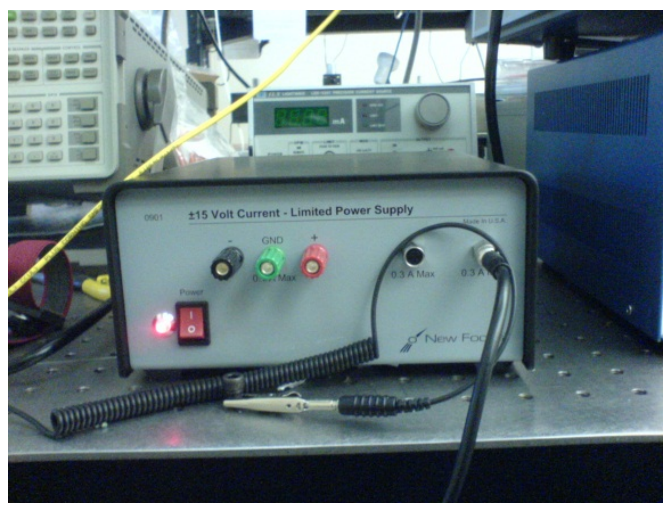

New Focus 901

+/- 15 Volt Current-Limited Power Supply for New Focus Broadband Amplifier 1422

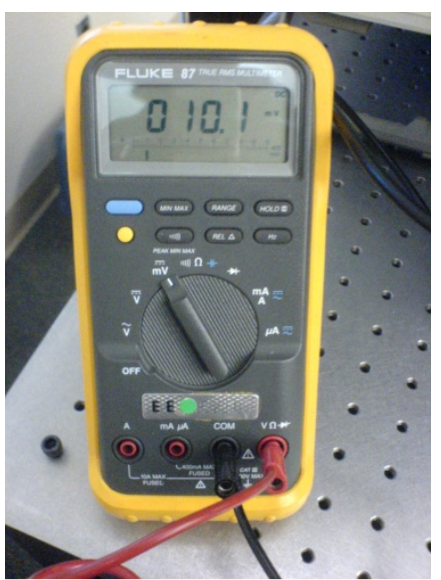

Fluke Digital Multi Meter 87

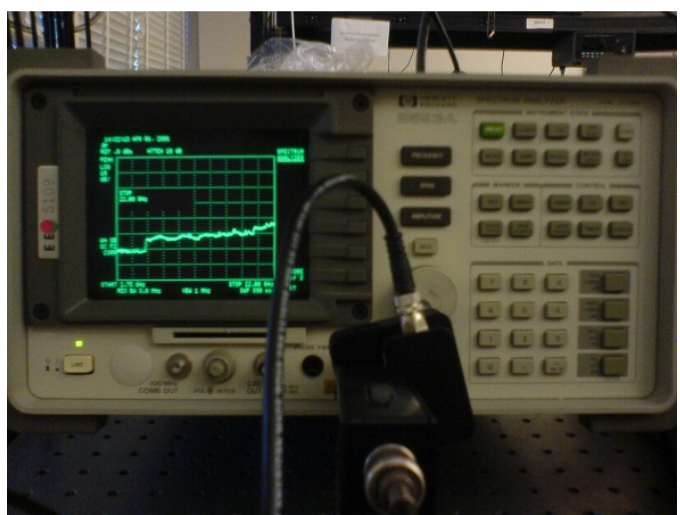

HP 8593A RF Spectrum Analyzer 


\section{REFERENCES}

[1] H. Li, K. Iga, "Vertical-Cavity Surface-Emitting Laser Devices", Springer, pp. 87, 2002

[2] J. C. Palais, "Fiber Optic Communications. $5^{\text {th }}$ Ed.", Pearson Prentice Hall, pp. 80, 2005.

[3] Honeywell, "HFE4192-901, 4.25 Gbps 850nm VCSEL LC TOSA", 2004

[4] E.P. O’Reilly and M. Silver, Appl. Phys. Lett. 63, pp. 3318 (1993).

[5] M.S.Ab-Rahman, M. S. D. Zan, M.F.A.S.Shaarani, "The Resolution Bandwidth Analysis of Data Acquisition in Optical Spectrum Analyzer and WDM Analyzer", IJCSNS, VOL.8 No.12, 2008.

[6] B. H. Ifr, an Aeroflex Company, "Selecting a Spectrum Analyzer", http://www2.electronicproducts.com/Selecting_a_spectrum_analyzer-articlejulifr1-jul2003.aspx, web, accessed April, 28, 2011

[7] “Optical Spectrum Analysis Application Note”, pp. 1550-4, Agilent Technologies

[8] M. A. Parker, "Physics of Optoelectronics. $5^{\text {th }}$ Edition", Taylor \& Francis, pp. 74, 2005.

[9] B. V. Zeghbroeck, "Principles of Semiconductor Devices. S.2.8", http://ecee.colorado.edu/ bart/book/book/chapter2/ch2_8.htm, web, accessed April, 28, 2011

[10] Professor's notes in RIN measurements, 2006

[11] K. Chang, "Microwave Solid-State Circuits and Applications", John Wiley \& Sons, Inc., pp.184-186, 1994. 
[12] C.H. Chang, C. J. Chang-Hasnain, "Injection Locking of VCSELs," IEEE J. Quantum Electron., Vol. 9, No. 5, pp. 1386-1393, 2003

[13] X. Jin, S.L. Chuang, "Relative Intensity Noise Characteristics of Injection-Locked Semiconductor Lasers,” Appl. Phys. Lett., Vol. 77, No. 9, pp. 1250-1252, 2000.

[14] X. Jin, S.L. Chuang, "Bandwidth Enhancement of Fabry-Pérot Quantum-Well Lasers by Injection-Locking", Solid-State Electronics, No. 50, pp. 1141-1149, 2006.

[15] H. Jeff, “The Laser Guidebook Second Edition”, McGraw-Hill, pp. 339, 1992. 